UNIVERSIDAD DE SALAMANCA FACULTAD DE CIENCIAS

VNiVERSIDAD Departamento de Física Fundamental

A SALAMANCA

\title{
CONSTRAINING ALTERNATIVE THEORIES OF GRAVITY WITH ASTROPHYSICAL AND COSMOLOGICAL DATASETS
}

\author{
PhD. Thesis \\ Memoria para optar al título de Doctor \\ Ivan de Martino
}

September 2014 

D. Fernando Atrio Barandela, Profesor Catedrático de la Universidad de Salamanca, y D. Salvatore Capozziello, Profesor Catedrático de la Universitá degli studi di Napoli "FEDERICO II"

CERTIFICAN:

Que el trabajo de investigación que se recoge en la siguiente memoria titulado "Constraining alternative theories of gravity with astrophysical and cosmological datasets", presentada por D. Ivan de Martino para optar al título de doctor y a la Mención de "Doctorado Internacional", ha sido realizada en su totalidad bajo su dirección y autorizan su presentación.

Septiembre, 2014

D. Fernando Atrio Barandela

Profesor Catedrático

Universidad de Salamanca

Prof. Salvatore Capozziello

Profesor Catedrático

Universitá degli studi di Napoli "FEDERICO II" 



\section{Publications}

The thesis is based on several papers developed during the last four years. From Ch. 2, chapters correspond to a published or submitted paper as follows

- Accepted for publication:

\section{Chapter 2}

$\checkmark$ Capozziello S., De Laurentis M., De Martino I., Formisano M., Odintsov $S . D$., 'Jeans analysis of self-gravitating systems in $f(R)$-gravity', Phys. Rev. D, 85, 044022, 2012.

\section{Chapter 3}

$\checkmark$ Mariafelicia De Laurentis, Ivan De Martino, 'Testing $f(R)$-theories using the first time derivative of the orbital period of the binary pulsars', MNRAS, 431, 741D, 2013.

\section{Chapter 4}

$\checkmark$ I. De Martino, M. de Laurentis, F. Atrio-Barandela, S. Capozziello, 'Constraining $f(R)$ gravity with Planck data on galaxy cluster, MNRAS, 442, 921, 2014.

\section{Chapter 5}

$\checkmark$ I. de Martino, F. Atrio-Barandela, A. da Silva, H. Ebeling, A. Kashlinsky, D. Kocevski, Carlos J.A.P. Martins, 'Measuring the redshift dependence of the CMB monopole temperature with PLANCK data. ', ApJ, 757, 144, 2012.

- Submitted to peer-review:

\section{Chapter 3}

$\checkmark$ Mariafelicia De Laurentis, Ivan De Martino, 'Probing f(R)-gravity by PSR J0348+0432', arXiv:1310.0711v2. 



\section{Contents}

Introduction: Motivation and Plan of the Thesis ix

0.1 Gravitational theories: from Einstein to the most recent results . . . . $\mathrm{x}$

0.2 Do we need to extend General Relativity? . . . . . . . . . . . . . . . xi

0.3 Brief Overview of the Current Work .............. . xii

1 The Evolving Universe: Theoretical and Observational Constraints 1

1.1 Brief introduction to General Relativity . . . . . . . . . . . . . 2

1.2 The Expanding Universe . . . . . . . . . . . . . . . . . . . 5

1.3 Cosmological Datasets to probe $\Lambda$ CDM model . . . . . . . . . . . . . . 9

1.4 Shortcomings of the concordance $\Lambda$ CDM model . . . . . . . . . . . . . 19

1.5 Extended Theories of Gravity . . . . . . . . . . . . . . . . . . . . . 20

$1.6 f(R)$ gravity: general formalism . . . . . . . . . . . . . . . . . . . . . 21

1.7 The weak field limit in $f(R)$-gravity. . . . . . . . . . . . . . . . 26

1.8 Discussion and remarks . . . . . . . . . . . . . . . . . . 30

2 Jeans Instability in $f(R)$ theories of gravity 31

2.1 Gravitational instability and star formation . . . . . . . . . . . . . 32

2.2 The Modified Poisson equation in $f(R)$-gravity . . . . . . . . . . . . . 35

2.3 Jeans criteria for gravitational instability in $f(R)$-gravity . . . . . . . . 36

2.4 The Jeans mass limit in $f(R)$-gravity . . . . . . . . . . . . . . . . . . 38

2.5 Discussion and future perspectives . . . . . . . . . . . . . . . 41

3 Gravitational Wave emission in Extended Theories of Gravity 45

3.1 Gravitational Waves: general framework and emission from binary systems 47

3.2 Energy-momentum tensor and quadrupolar emission in $f(R)$-gravity . 51

3.3 First time derivative of the orbital period in $f(R)$-gravity . . . . . . 52

3.4 Constraints on ETGs from binary systems . . . . . . . . . . . . . . . 53

3.5 Massive or massless gravitons? . . . . . . . . . . . . . . . . . . 57

3.6 Conclusions and future perspectives . . . . . . . . . . . . . . . 58 
4 Cluster pressure profiles in Extended Theory of gravity 61

4.1 The Sunyaev-Zel'dovich effect: cluster of galaxies . . . . . . . . . . . 63

$4.2 f(R)$ pressure profile . . . . . . . . . . . . . . 70

4.3 Data and methodology . . . . . . . . . . . . . . . . 71

4.4 Estimation of the $f(R)$ parameters . . . . . . . . . . . 77

4.5 Discussion and future perspectives . . . . . . . . . . . . 79

5 Redshift evolution of the CMB blackbody temperature $\quad 81$

5.1 Constraining redshift evolution of CMB temperature . . . . . . . . . . 83

5.2 Cluster templates and final maps . . . . . . . . . . . . . . . . . . 88

5.3 Data analysis and results . . . . . . . . . . . . . . . . . 92

5.4 Discussion and future perspectives . . . . . . . . . . . . . . 103

$\begin{array}{ll}\text { A Fundamental constants and conversion factors } & 109\end{array}$

B Yukawa-like correction to Newtonian gravitational potential 111

$\begin{array}{ll}\text { C Solutions of unstable modes of a stellar system } & 121\end{array}$

D Post-Minkowskian limit and quadrupolar emission of gravitational waves in $f(R)$ gravity

E Relativistic corrections of thermal Sunyaev-Zeldovich effect 133 


\section{Acronyms}

$\begin{array}{ll}\text { 2dF } & \text { Two-degree-Field Galaxy Redshift Survey } \\ \text { BHs } & \text { Black Holes } \\ \text { CL } & \text { Confidence Level } \\ \text { CMB } & \text { Cosmic Microwave Background } \\ \text { DE } & \text { Dark Energy } \\ \text { DM } & \text { Dark Matter } \\ \text { ETGs } & \text { Extend Theories of Gravity } \\ \text { FLRW } & \text { Friedmann-Lemaitre-Robertson-Walker metric } \\ \text { GR } & \text { General Relativity } \\ \text { GWs } & \text { Gravitational Waves } \\ \text { HFI } & \text { High Frequency Instrument } \\ \text { ICM } & \text { Intra Cluster Medium } \\ \text { ISM } & \text { Inter Stellar Medium } \\ \text { ILC } & \text { Internal Linear Combination } \\ \text { KSZ } & \text { Kinetic Sunyaev-Zeldovich } \\ \text { LG } & \text { Local Group } \\ \text { LSS } & \text { Large Scale Structure } \\ \text { LFI } & \text { Low Frequency Instrument } \\ \text { LHC } & \text { Large Hadron Collider } \\ \Lambda \text { CDM } & \text { Lambda Cold Dark Matter } \\ \text { NFW } & \text { Navarro-Frenk-White } \\ \text { NSs } & \text { Neutron Stars } \\ \text { QM } & \text { Quantum Mechanics } \\ \text { QFT } & \text { Quantum Field Theory } \\ \text { SDSS } & \text { Sloan Digital Sky Survey } \\ \text { SPT } & \text { South Pole Telescope } \\ \text { SR } & \text { Special Relativity } \\ \text { SN } & \text { SuperNovae } \\ \text { SNeIa } & \text { SuperNovae Type Ia } \\ \text { SZ } & \text { Sunyaev-Zeldovich } \\ \text { TSZ } & \text { Thermal Sunyaev-Zeldovich } \\ \text { WDs } & \text { White Dwarfs } \\ \text { WMAP } & \text { Wilkinson Microwave Anisotropy Probe } \\ & \end{array}$




\section{Notations and symbols}

We consider a four-dimensional pseudo-Riemannian spacetime given by a manifold $\mathcal{M}$ and a metric $g_{\mu \nu}$. The signature is $(-+++)$ or $(+---)$ depending on the phenomena we are studying, in according to the most common one used in literature.

$\begin{array}{ll}x^{\mu}=\left(t, x^{1}, x^{2}, x^{3}\right) & \text { Coordinate convention for General Relativity and Extended } \\ & \text { Theories of Gravity } \\ d_{A}(z) & \text { Angular diameter distance } \\ d_{L}(z) & \text { Luminosity distance } \\ f^{(0)} & \text { Distribution function } \\ f^{(1)} & \text { Distribution function at equilibrium } \\ f_{G} & \text { Distribution function at first order of perturbation } \\ g(\nu) & \text { Gravitational constant } \\ g_{\mu \nu} & \text { SZ frequency dependence for cluster of galaxies } \\ h_{\mu \nu} & \text { Metric tensor } \\ h_{i j}^{T T} & \text { First order perturbation to metric tensor } \\ H_{0} & \text { Traceless-transverse tensor } \\ H(z) & \text { Current value of Hubble constant } \\ k_{B} & \text { Expansion rate of the Universe } \\ M_{J} & \text { Boltzmann constant } \\ M_{v i r} & \text { Jeans Mass } \\ M_{500} & \text { Virial Mass } \\ n_{e}(r) & \text { Cluster Mass at radius } r_{500} \\ m_{e} & \text { Electron density } \\ m_{p} & \text { electron mass } \\ P_{b} & \text { proton mass } \\ \dot{P}_{b} & \text { Orbital period of binary system } \\ R_{\mu \nu} & \text { First time derivative of the orbital period of binary system } \\ R & \text { Ricci tensor } \\ R_{\mu \nu \beta}^{\alpha} & \text { Riemann tensor } \\ & \end{array}$


$r_{500} \quad$ Radius at which the density is 500 times the critical density of the Universe

$r_{v i r} \quad$ Virial radius

$T_{e}(r) \quad$ Electron temperature

$T_{0} \quad$ CMB temperature at $z=0$

$T_{\mu \nu} \quad$ Stress-Energy tensor

$x_{\mu} \quad$ Coordinates of the reference frame

$y_{c} \quad$ Comptonization parameter

$\lambda_{J} \quad$ Jeans length

$\eta_{\mu \nu} \quad$ Minkowski metric

$\rho_{c} \quad$ Critical density of the Universe

$\sigma_{T} \quad$ Thomson cross section

$\theta_{500} \quad$ Angle subtended by a cluster of radius $r_{500}$

$\Gamma_{\mu \nu}^{\alpha} \quad$ Christoffel symbol (affine connections)

$\Lambda \quad$ Cosmological Constant

$\Omega_{b}(z) \quad$ Baryon density

$\Omega_{b}^{(0)} \quad$ Current value of baryon density

$\Omega_{k}(z) \quad$ Curvature density

$\Omega_{k}^{(0)} \quad$ Current value of curvature density

$\Omega_{m}(z) \quad$ Matter density

$\Omega_{m}^{(0)} \quad$ Current value of matter density

$\Omega_{r}(z) \quad$ Radiation density

$\Omega_{r}^{(0)} \quad$ Current value of Radiation density

$\Omega_{\Lambda}(z) \quad$ Dark energy density

$\Omega_{\Lambda}^{(0)} \quad$ Current value of dark energy density 


\section{Introduction: Motivation and Plan of the Thesis}

\section{Contents}

0.1 Gravitational theories: from Einstein to the most recent

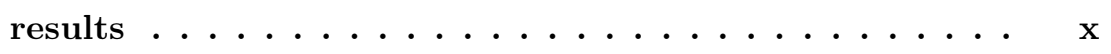

0.2 Do we need to extend General Relativity? . . . . . . . xi

0.3 Brief Overview of the Current Work ......... xii

he most ancient branch of science is probably Cosmology (from the Greek: kosmos,
Universe, world, order and logos, word, theory). Early civilizations used their own cosmological models to establish the seasons and made detailed astronomical observations that were very useful to predict periodic phenomena like rainy seasons and periods of droughts. The knowledge about the kosmos was increased through the centuries but quantitatively our understanding did not go further than to give orders of magnitude estimates of the quantities involved. Nevertheless, observations have quickly improved and became more accurate in the last three decades allowing us to obtain better measurements of the basic cosmological parameters. We have entered the era of precision cosmology where the observables have been determined within a few percent accuracy (from the Wilkinson Microwave Anisotropy Probe (WMAP) and Planck satellite data and the Sloan Digital Sky Survey (SDSS), among other data sets). The data are well accommodated within the framework of the Standard Cosmological Model or Concordance Model, based on the Big Bang Theory together with the inflationary paradigm. The model explains the evolution of the Universe from the first fractions of a second to the present day. The Concordance Model is based on General Relativity (GR) and is supported by three main observations: the expansion of the Universe, discovered by E. P. Hubble, the relative abundance of light elements, explained by G. Gamow and the Cosmic Microwave Background (CMB) radiation, discovered by A. A. Penzias and R. W. Wilson. Each of these four elements dates back to at least fifty years. In the last two decades new observational data have emerged, confirming the model further but also have pointed out some shortcomings that question if GR is the effective theory of gravity. 
Is GR sufficient to explain all the gravitational phenomena from collapsed objects to the evolution of the Universe and the formation of structures? Does the theory need to be changed, or at least, modified? These are still open questions and two main approaches have been taken: (A) preserve the success of GR by incorporating new particles and/or scalar fields not yet observed and improve the accuracy of the data to verify the model further and (B) modify the theory of gravity to make it compatible with Quantum Mechanics and cosmological observations without introducing additional particles and fields. Independently of the preferred approach, the main goal of this thesis is to test GR and its alternatives at all scales, ranging from scales of a few astronomical units to cosmological scales in order to constrain-confirm-rule out the theories that can be used to describe the observed Universe.

\subsection{Gravitational theories: from Einstein to the most re- cent results}

In the first two decades of the XXth century, Einstein developed the Special and General Theories of Relativity (Einstein (1905a,b, 1916)), that lay the foundation of the study of the Universe. Both theories introduced the concept of the space-time as a unique dynamical entity. GR provides the theoretical foundation to describe the evolution of the Universe and is the basis of the Standard Cosmological Model. This model has been successfully tested using many different and independent observations. The accelerated expansion of the Universe was first established using luminosity distances derived from observations of SuperNovae type Ia (SNeIa) (Perlmutter et al. (1997), Riess et al. (2004), Astier et al. (2006), Clocchiati (2006), Suzuki et al. (2012)). For this discovery, S. Perlmutter (University of Berkley, USA), B.P. Schmidt (National University in Weston Creek, Australia) and A.G. Riess (University of Baltimore, USA) were awarded the Nobel Prize in 2011. Next, measurements of temperature fluctuations on the CMB radiation have allowed to estimate cosmological parameters with very high accuracy (Hinshaw et al. (2013), Planck Collaboration (2013i,j,l,m)). Other independent probes came from the power spectrum of matter density perturbations measured from the 2-degree field (2dF) survey of galaxies (Percival et al., 2001, Pope et al., 2004), the Sloan Digital Sky Survey (SDSS) (Tegmark et al., 2004), among other probes of the concordance model.

The last confirmation of the Standard Cosmological Model comes from the BICEP 2 results (BICEP2 Collaboration, 2014). The measurement of the B-mode of the CMB polarization has revealed for the first time the presence of primordial Gravitational Waves (GWs) originated during the first fractions of a second in the life of the Universe. These primordial GWs would have originated during the inflationary period proposed to solve the problems of horizon and flatness (Kazanas, 1980, Guth, 1981). If these 
results stand further confirmation, they will represent the first observational proof of inflation, a basic tenet of the Standard Cosmological Model.

The recent observations, of which Planck and BICEP 2 represent the latest two exponents, show that we have entered a new era in cosmology. Precise measurements have already modified our knowledge of the Universe. In the future, new experiments will provide more accurate measurements of the Large Scale Structure (LSS) of the Universe, CMB polarization and distortions, GWs and many others, that will allow us to put tighter constraints on the parameter space of cosmological models.

\subsection{Do we need to extend General Relativity?}

Seeing the great successes of GR in the description of gravitational phenomena ranging from collapsed objects such as White Dwarfs (WDs) and Neutron Stars (NSs) to cosmological scales, it is important to ask if there is any need to change, modify or extend our current theory of gravity. Back in the 1920s there was a need to modify gravity in order to unify it with other interactions (see for example at Weyl (1918), Pauli (1919), Eddington (1924), Lanczos (1931)). In fact, despite its many successes, GR is not a Quantum Theory and it can not provided a description of the Universe at the quantum scales. Many efforts have been devoted to unify the Quantum Field Theory and General Relativity with little success. Within GR it is not possible to explain the emergence of the Large Scale Structure and the acceleration of the Universe without introducing two unknown components called Dark Matter (DM) and Dark Energy (DE), respectively. Our knowledge about these two ingredients of the cosmic energy-density budget comes from to their dynamical effects, but their fundamental nature, whether particles or scalar fields is completely unknown.

The need for requiring two unknown matter components to fully explain the observations within a GR framework has been interpreted as breakdown of the theory at astrophysical and cosmological scales (Capozziello and De Laurentis, 2011). Further, whatever was the unification scheme one has in mind, let it be Superstrings, Supergravity or Grand Unified Theories, they all consider non-minimal couplings to the geometry and higher order terms in the curvature invariants to be present in the effective Lagrangian. Thus, it is important to explore if those extensions of GR overcome the previous shortcomings. Instead of adding extra components in the stress-energy tensor, one could change the geometrical description in the Hilbert-Einstein Lagrangian. In the most general approach, the Lagrangian would be

$$
\mathcal{L}=F\left(R, \square R, \square^{2} R, . . \square^{k} R, \phi\right)-\frac{\epsilon}{2} g^{\mu \nu} \phi_{; \mu} \phi_{; \nu}+2 \kappa \mathcal{L}^{(m)}
$$

where $F$ represents a generic function of curvature invariants, $\kappa$ the coupling constant, $\phi$ the scalar field and its nature and dynamics (standard, phantom or no-dynamical 
field) are specified by $\epsilon$. There are no a priori reasons to restrict the gravitational Lagrangian to be a linear function of the Ricci scalar $R$, and minimally coupled with matter. Higher order terms give rise to contributions of order two in the field equations, that can be read as additional degrees of freedom in the theory of gravity or additional scalar fields as one prefers (Capozziello and De Laurentis, 2011). Throughout this thesis, we will just concentrate our attention on a particular family of Extended Theories of Gravity (ETGs), called $f(R)$-theories, that give a general and straightforward prescription to extend the theory of gravitation by replacing the Hilbert-Einstein Lagrangian with a general function of Ricci curvature, $f(R)$. However, to be sure about their capacity to explain the gravitational interaction as well as GR, the ETGs need to be tested in all possible astronomical scenarios in order to understand at which scales their contributions are significant.

\subsection{Brief Overview of the Current Work}

The layout of the $\mathrm{PhD}$ thesis is organized as follows. In Chapter 1 we provide the necessary background information needed to describe the problems addressed in this thesis. GR is introduced prior to describe its extensions. Cosmological implications in standard gravity, and shortcomings of GR will be discussed to make a brief introduction to ETGs, centering our attention on $f(R)$ gravity and its weak field limit to set the frame for our original research.

In Chapter 2 we will review how stars form in GR, and then we will study how star formation is modified in the context of $f(R)$. The mechanism that drives the formation of the structure is Jeans instability, so that in this chapter we will analyze the dynamics and collapse of collisionless self-gravitating systems in $f(R)$-gravity in the weak field approximation. We will describe a system at equilibrium by a time-independent distribution function $f(\vec{r} ; v)$ whose evolution is described by collisionless Boltzmann equation and two potentials $\Phi_{0}(\vec{r})$ and $\Psi_{0}(\vec{r})$, solutions of the modified Poisson equation. We will discuss the evolution of density perturbations on a homogeneous and neutral system of dust particles in linear theory.

In Chapter 3 we will analyze the Gravitational Waves emission of a binary system to test the strong field regime on scales of a few Astronomical Units. We will discuss the mechanism of GWs emission in GR before presenting an analytic solution for the quadrupolar emission in $f(R)$-gravity and the first time derivative of the orbital period, the best measured Post-Keplerian parameters. We will compare our predictions with measurements of a sample of relativistic binary systems to constrain the theory of gravity.

While $f(R)$ gravity models are well tested at galaxy scales - see for instance, Capozziello et al. (2007), Cardone and Capozziello (2011), Napolitano et al. (2012) 
-, in Chapter 4 we will introduce a new test on a slightly larger scale, that of clusters of galaxies using the thermal Sunyaev-Zeldovich (TSZ) anisotropies induced by the hot ionized gas residing in the potential wells of clusters of galaxies. We will compute the pressure profile of 579 clusters of galaxies assuming that the ionized gas is in hydrostatic equilibrium within the potential well of the modified gravitational field, without resorting to any DM component. We will compare the predicted profiles with those measured using the foreground clean SMICA map produced by the Planck collaboration. We will show that alternative theories of gravity represent an adequate fit to the data. We will establish that the cluster potential wells can not be dominated by baryons alone and that either DM or a modification of GR is required to explain their pressure profiles.

In Chapter 5 we will test the Standard Cosmological Model by measuring the scaling of the CMB blackbody temperature with redshift by means of the TSZ anisotropies. We will forecast the capability of Planck data to constrain deviations from adiabatic evolution, measured in terms of a parameter $\alpha$ as $T(z)=T_{0}(1+z)^{1-\alpha}$. Among our results we find that $\mathrm{CMB}$ data alone can constrain deviations of adiabatic evolution to the 1-2\% level. These results represent a factor of 2-3 improvement over similar measurements carried out using quasar spectral lines and a factor 6-20 with respect to earlier results using smaller cluster samples.

Finally, we will present our main conclusion and suggest some future perspectives in the field. 


\section{$\Gamma_{\text {Chapter }} 1$}

\section{The Evolving Universe: Theoretical and Observational Constraints}

\section{Contents}

1.1 Brief introduction to General Relativity . . . . . . . . 2

1.2 The Expanding Universe .............. 5

1.2.1 Homogeneous and isotropic Universe . . . . . . . . . . . 6

1.2.2 The $\Lambda$ Cold Dark Matter Cosmological Model . . . . . . . . . 8

1.3 Cosmological Datasets to probe $\Lambda$ CDM model . . . . . 9

1.3.1 The cosmic distances . . . . . . . . . . . . . . 10

1.3.2 The Type Ia Supernovae . . . . . . . . . . . . . . . . . . . 12

1.3.3 The Cosmic Microwave Background radiation . . . . . . . . . 12

1.4 Shortcomings of the concordance $\Lambda$ CDM model . . . . . 19

1.5 Extended Theories of Gravity . . . . . . . . . . 20

$1.6 f(R)$ gravity: general formalism ............. 21

1.6.1 Formalisms and frames in $f(R)$ gravity . . . . . . . . . 23

1.6.2 $f(R)$-models . . . . . . . . . . . . . . 24

1.6.3 The chameleon mechanism in $f(R)$ gravity . . . . . . . 25

1.7 The weak field limit in $f(R)$-gravity. . . . . . . 26

1.7.1 Weak field limits . . . . . . . . . . . . . . . 26

1.7.2 The Post-Newtonian limits . . . . . . . . . . . . . . 28

1.8 Discussion and remarks ............... 30

n the XVII century G. Bruno, G. Galilei and R. Descartes expressed, for the first
time, the idea that space and time can be the object of scientific research, becoming the scenario where the physical theories are to be constructed. Later, in the scientific opus Philosophiae Naturalis Principia Mathematica, Sir Isaac Newton defined space and time as absolute objects. In parallel, Gottfried Wilhelm von Leibniz defined space and time relatively to objects and events. Historically, the Newtonian vision was the dominant one till the beginning of the XXth century. The Newtonian theory 
of gravity provided a very good description of the dynamics of celestial bodies; it applied the principles of Classical Mechanics to astronomical objects such as stars and planets. It provided astronomers with the tools to compute ephemeris. Its successes were limited to describe the nearby Universe, using an euclidean three dimensional reference frame. It showed its shortcomings when the theory could not explain the "anomalous" precession of the perihelion of Mercury discovered by Le Verrier (1859).

In the first two decades of XXth century, A. Einstein unified the concepts of space and time, clarifying that they are not absolute entities, as in Classical Mechanics, but dynamical quantities related to the distribution of matter and energy. The first step of this revolution was represented by the publication of the Theory of Special Relativity (SR) in the 1905 (Einstein (1905a,b), followed by the formulation of the General Relativity (GR) in the 1915 (Einstein, 1916). Both theories were based on previous studies by physicists like J.C. Maxwell, E. Mach, H.A. Lorentz and mathematicians like C.F. Gauss and B. Riemann. This approach led to a new conception of the Universe itself, now considered as a dynamical system. The equations of motion developed in GR explained away the discrepancy between the measured precession of the perihelion of Mercury with Newtonian gravity. Also, they predicted a value for deflection of light due to a gravitational field that was observationally verified by A. Eddington in 1919 (Hoskin, 1999). The field equations of GR also described the evolution of Universe as a whole. Modern Astrophysics and Cosmology are entirely based on GR and the cosmological solutions have been confirmed by observations carried out in the last decades. However, some inconsistencies have arisen when describing the emergence and evolution of the Large Scale Structure in the Universe. In the standard approach, these inconsistencies are solved by introducing two new components, Dark Matter (DM) and Dark Energy (DE). Alternatively, the theory of GR can be extended to generate more complex theories of gravitation that lead to field equations of higher order than those of Einstein.

In this chapter, we will introduce the basic concepts needed in the subsequent development of the thesis. First, we will review the theory of GR and the Standard Cosmological Model, and we will describe the datasets that have shaped our understanding of the Universe. Next, we will describe alternative theories of gravity paying particular attention to $f(R)$-gravity. We will review some basic concepts of these theories and their weak field limit. Finally, we will summarize the successes and shortcomings of the Standard Cosmological Model, and we will indicate the motivation for considering Extended Theories of Gravity (ETGs) as an alternative to GR.

\subsection{Brief introduction to General Relativity}

In 1905, Einstein published the Theory of Special Relativity (Einstein (1905a,b)), 
where he studied the transformation of inertial systems in a Minkowskian space-time. The underlying assumption was that no physical interaction can propagate faster than the speed of light $c$, hypothesis that contradicted the dominant Newtonian vision of action at a distance. Later, Einstein posed and solved the problem of how to make compatible gravity with Special Relativity and formulated the Theory of General Relativity in the 1916 (Einstein, 1916). This theory is based on the Equivalence Principle in which accelerated systems are locally indistinguishable from a gravitational fields. This principle was supported by experimental measurements made by Eötvös at the end of XIX century, that established the equivalence between the inertial and gravitational masses. The new theory of gravitation provided a mathematical expression to this principle, and drew important consequences for Astrophysics and Cosmology.

In GR the distance between two neighboring space-time events is given in terms of a quadratic form of the coordinates:

$$
d s^{2}=g_{\mu \nu} d x^{\mu} d x^{\nu}
$$

where the metric tensor $g_{\mu \nu}$ is a function of the space-time coordinates $\left(x^{\mu}\right)$. From here on, we will denoted with "," partial derivative and with " ;" covariant derivative with regard to $g_{\mu \nu}$; all Greek indices will run from $0, \ldots, 3$ and Latin indices from $1, \ldots, 3 ; g$ will indicate the determinant of the metric.

The motion of a free particle is determined by the action

$$
\mathcal{S}_{p m}=-m c \int_{s_{1}}^{s_{2}} \mathrm{~d} s .
$$

The extremal of this action, $\delta S_{p m}=0$, gives the equation of geodesics, i.e., the curve of minimum distance connecting two space-time events:

$$
\frac{d^{2} x^{\alpha}}{d s^{2}}+\Gamma_{\mu \nu}^{\alpha} \frac{d x^{\mu}}{d s} \frac{d x^{\nu}}{d s}=0
$$

where $\Gamma_{\mu \nu}$ are the Christoffel symbols, also called affine connections, defined as

$$
\Gamma_{\mu \nu}^{\alpha}=\frac{1}{2} g^{\alpha \lambda}\left(g_{\lambda \mu, \nu}+g_{\nu \lambda, \mu}-g_{\mu \nu, \lambda}\right)
$$

In GR, geodesics represent the trajectories of free-falling particles on a gravitational field.

In order to derive the equations describing the gravitational field, Einstein had to describe, first of all, the intrinsic curvature of the space-time. Intuitively, the curvature is a measure of how a trajectory deviates from its tangent. That is, curvature is a local property. While our perception of curvature is extrinsic, i.e., we perceive the curvature of a surface if it is embedded on a three dimensional space, Gauss discovered that in reality is an intrinsic property of the surface that can be measured without having to resort to higher dimensions. The curvature could be described by the Riemann tensor,

$$
R_{\mu \beta \nu}^{\alpha}=\Gamma_{\mu \beta, \nu}^{\alpha}-\Gamma_{\mu \nu, \beta}^{\alpha}+\Gamma_{\mu \beta}^{\lambda} \Gamma_{\lambda \nu}^{\alpha}-\Gamma_{\mu \nu}^{\lambda} \Gamma_{\lambda \beta}^{\alpha}
$$


This tensor verifies the Bianchi identities

$$
R_{\mu \beta \nu ; \lambda}^{\alpha}+R_{\beta \nu \lambda ; \mu}^{\alpha}+R_{\nu \lambda \mu ; \beta}^{\alpha}=0
$$

Contracting the first and third index of the Riemann tensor yields the Ricci tensor

$$
R_{\mu \nu}=\partial_{\sigma} \Gamma_{\mu \nu}^{\sigma}-\frac{\partial^{2} \ln \sqrt{-g}}{\partial x^{\mu} \partial x^{\nu}}+\Gamma_{\tau \sigma}^{\sigma} \Gamma_{\mu \nu}^{\tau}-\Gamma_{\alpha \sigma}^{\tau} \Gamma_{\tau \nu}^{\sigma}
$$

Its trace

$$
R=g^{\mu \nu} R_{\mu \nu},
$$

is known as the scalar curvature or Ricci Scalar.

The equations describing the gravitational interaction must be written in tensorial (covariant) form and, in the adequate limit, they must recover Newton's theory. The field equations can be derived from the following action

$$
\mathcal{S}=\int \mathrm{d}^{4} x \sqrt{-g}\left(\frac{1}{c} \mathcal{L}_{m}+\frac{c^{3}}{16 \pi G} R\right)
$$

where $\mathcal{L}_{m}$ is the matter Lagrangian. The variation of the matter Lagrangian gives rise to the stress-energy tensor

$$
\frac{1}{2} \sqrt{-g} T^{\mu \nu}=\frac{\partial}{\partial g_{\mu \nu}}\left(\sqrt{-g} \mathcal{L}_{m}\right)-\frac{\partial}{\partial x^{\mu}} \frac{\partial}{\partial g_{\mu \nu, \mu}}\left(\sqrt{-g} \mathcal{L}_{m}\right),
$$

that describes the matter content of the system. The field equations can be derived by the extremal of eq. (1.9) (Weinberg, 1972)

$$
R_{\mu \nu}-\frac{1}{2} g_{\mu \nu} R=\frac{8 \pi G}{c^{4}} T_{\mu \nu},
$$

that shows that the structure of the space-time is determined by the matter-energy distribution of the system.

GR explains many new effects with important astrophysical implications (Will, 2006). Some can be directly derived from the equivalence principle (Uzan (2003), Williams et al. (2004)). Photons are blueshifted/redshifted when falling/emerging from a gravitational potential well. The gravitational redshift is well established by measurements in the laboratory and using astronomical observations (Wojtak et al. (2011), Zhao et al. (2013)). The deflection of light due to the gravitational fields of massive bodies has been confirmed by observing the effect of the Sun on the light of background stars or distant quasars (Fomalont and Kopeikin (2003), Kopeikin and Fomalont (2007)). The gravitational time delay (or Shapiro delay) of photons has been tested successfully (Bertotti et al. (2003), Fomalont et al. (2010)).

In analogy to electromagnetic waves, the theory predicts the existence of Gravitational Waves (GWs), ripples of the space-time that propagate at the speed of light. Their existence has been established indirectly from various pulsar timing arrays. 
Ground based observatories like LIGO and VIRGO are searching the GWs emission from compact objects using laser interferometry. Recently, NASA/ESA have accepted the L-class mission eLISA ${ }^{1}$ (Amaro-Seoane et al., 2013) a redesign of the Laser Interferometer Space Antenna (LISA) proposal to measure gravitational waves from space.

Finally, GR is the basis of the current cosmological model, and it makes important predictions that are widely verified by many independent observations: CMB temperature fluctuations (Hinshaw et al. (2013), Planck Collaboration (2013j)), matter density perturbation (Percival et al. (2001), Pope et al. (2004), Tegmark et al. (2004), luminosity distances from SNeIa (Riess et al. (1998), Perlmutter et al. (1999), Riess et al. (2004), Astier et al. (2006), Davis et al. (2007), Kowalski et al. (2008), Amanullah et al. (2010), Suzuki et al. (2012)) and the expansion rate of the Universe (Jimenez et al. (2003), Simon et al. (2005), Stern et al. (2010), Moresco et al. (2012a,b)).

\subsection{The Expanding Universe}

Not until the pioneering observations of E.P. Hubble showed the Universe to be expanding, the Universe was assumed to be static. Previously, Einstein had introduced the cosmological constant term $\Lambda g_{\mu \nu}$ to derive static solutions from the field equations. Between 1922 and 1924, A. Friedman showed that GR contained solutions describing an expanding Universe. Independently, G.E. Lemaître found similar results, and finally, in 1935, H.P. Robertson and A.G. Walker showed that for an homogeneous and isotropic space-time this solution is unique. In the meantime, Hubble discovered that the recession velocity $v_{r}$ of a galaxy was proportional to the distance $d$ from the observer

$$
v_{r}=H_{0} d
$$

Here, $H_{0}$ is the Hubble constant, $v_{r}$ is the velocity recession along the line of sight, and $d$ is the distance of the object from the observer. The main idea was that the radiation from galaxies is redshifted due to the general expansion of the Universe. After this discovery, Einstein eliminated the cosmological constant term $\Lambda$ from its equations accepting the idea that the Universe was not static. Nevertheless, astronomers considered it to be a parameter that had to be determined observationally and continued to discuss different cosmologies including positive (repulsive) and negative (attractive) cosmological terms.

If the Universe is homogeneous and isotropic, it is possible define a co-moving coordinates system in which the system of coordinates is fixed with respect to the overall Hubble flow of the Universe. The physical distance $d$ between two different

\footnotetext{
${ }^{1}$ https://www.elisascience.org/
} 
points is related to the co-moving distance $l$ through a scale factor $a(t)$ as

$$
d(t)=a(t) l .
$$

The scale factor can be determined from frequency shift of photons emitted by the distant sources. The cosmological redshift is defined as (Weinberg, 1972)

$$
z \equiv \frac{\lambda_{0}-\lambda_{e}}{\lambda_{e}}
$$

where, $\lambda_{0}$ is the observed wavelength of the photons, and $\lambda_{e}$ is the wavelength measured in the laboratory. Therefore, one can write

$$
z \equiv \frac{\lambda_{0}-\lambda_{e}}{\lambda_{e}}=\frac{\lambda_{0}}{\lambda_{e}}-1=\frac{a\left(t_{0}\right)}{a\left(t_{e}\right)}-1=\frac{a\left(t_{0}\right)-a\left(t_{e}\right)}{a\left(t_{e}\right)}>0,
$$

that shows that the redshift is due to the expansion of the Universe.

\subsubsection{Homogeneous and isotropic Universe}

In spherical coordinates, the Friedman-Lemaittre-Robertson-Walker (FLRW) metric that represents the line element of an homogeneous and isotropic Universe is (Peacock, 1999)

$$
d s^{2}=c^{2} d t^{2}-a^{2}(t)\left[d r^{2}+\chi(r)^{2}\left(\sin ^{2} \theta, d \phi^{2}+d \theta^{2}\right)\right]
$$

where $a(t)$ is the scale factor, and $\chi(r)$ depends on the spatial curvature of the metric

$$
\chi(r)=\left\{\begin{array}{llll}
r & \text { if } & k=0 & \text { (flat Universe), } \\
\sin r & \text { if } & k=+1 & \text { (closed Universe) } \\
\sinh r & \text { if } & k=-1 & \text { (opened Universe) }
\end{array}\right.
$$

The actual curvature depends on the energy density of the Universe. If large, equal or smaller than the critical value

$$
\rho_{c}=\frac{3 H_{0}^{2}}{8 \pi G}
$$

then the Universe will be close, flat or open. Here, $H_{0}=(67.4 \pm 1.4) \mathrm{km} \mathrm{s}^{-1} \mathrm{Mpc}^{-1}$ (Planck Collaboration, 2013j). Alternatively, $H_{0}=100 h \mathrm{~km} \mathrm{~s}^{-1} \mathrm{Mpc}^{-1}$ ) and $\rho_{c} \sim$ $2.7 \cdot 10^{11} M_{\odot} h^{-1} \mathrm{Mpc}^{3}$. If the total matter and energy density of the Universe, $\rho_{t o t}$, is less than the critical value then the gravitational force will not be sufficient to halt the expansion and the Universe will expand indefinitely. When the density exceeds the critical value, the Universe will stop expanding and will collapse. If the density is equal to the critical value, then the Universe will expand indefinitely with a speed going asymptotically to zero.

To complete the description of the Universe, one needs to specify the energymomentum tensor describing the energy distribution of the different matter components. The simplest model is that of a perfect fluid, characterized by a density $\rho$ and a isotropic pressure $p$; in this case, the energy-momentum tensor is

$$
T_{\mu \nu}=(\rho+p) u_{\mu} u_{\nu}-p g_{\mu \nu} .
$$


Density and pressure are usually related through the equation of state,

$$
w=\frac{p}{\rho}
$$

The trace of energy-momentum tensor is $\operatorname{tr}\left\{T_{\mu \nu}\right\}=\rho c^{2}-3 p$. Using the eqs. (1.11), (1.16), and (1.19), one can derive the Friedmann Equations, the equations that describe the evolution Universe as a whole

$$
\begin{aligned}
& H^{2}=\frac{8 \pi G}{3 c^{2}} \rho+\frac{\Lambda c^{2}}{3}-\frac{K c^{2}}{a^{2}}, \\
& 3 H^{2}+2 \dot{H}=-\frac{4 \pi G}{3 c^{2}}(\rho+3 p)+\frac{\Lambda c^{2}}{3}
\end{aligned}
$$

where $\Lambda$ is the cosmological constant term. The expansion rate of the Universe can be written in terms of the scale factor as

$$
H(t) \equiv \frac{\dot{a}(t)}{a(t)}
$$

These equations describe the evolution of the average properties of the Universe in the Standard Cosmological Model. Eq. (1.21) reduces to

$$
1=\frac{8 \pi G}{3 H(t)^{2}} \rho(t)+\frac{\Lambda}{3 H(t)^{2}}-\frac{k}{H(t)^{2} a^{2}(t)} .
$$

This expression shows the different components of the energy budget in units of the critical density. It is customary to denote

$$
\begin{aligned}
& \Omega_{m}(t)=\frac{8 \pi G}{3 H(t)^{2}} \rho(t)=\frac{\rho(t)}{\rho_{c}}, \\
& \Omega_{k}(t)=-\frac{k}{H(t)^{2} a^{2}(t)}, \\
& \Omega_{\Lambda}(t)=\frac{\Lambda}{3 H(t)^{2}}
\end{aligned}
$$

where $\Omega_{m}(t)$ is the amount of energy density due to the matter, and it is generally divided into baryonic $\Omega_{b}$ and dark matter $\Omega_{D M}$ components. $\Omega_{k}(t)$ and $\Omega_{\Lambda}(t)$ are the energy densities associated with the curvature of the Universe and the cosmological constant. The cosmological constant represents a particular case $(w=-1)$ of a more general class of fluids, termed DE for which the equation of state parameter could be a function of redshift, and is chose to be $w_{X} \leq-1 / 3$ to produce an accelerated expansion (Peebles and Ratra, 2003).

An immediate consequence of the two Friedmann equations is the continuity equation, that could also be derived from the conservation of the energy-momentum tensor. It can be written as

$$
\dot{\rho}+3 H(\rho+P)=0 .
$$


Integrating the previous equation one obtains

$$
\rho \propto a^{-3(1+w)}
$$

Therefore, the evolution of the energy densities depends on the nature of the fluid as specified by the equation of state parameter. A summary of the different fluids considered in this thesis is given in Table1.1.

\begin{tabular}{|lccc|}
\hline \hline Component & $w$ & $\rho(a(t))$ & $a(t)$ \\
\hline \hline non-relativistic matter & 0 & $\propto a^{-3}$ & $t^{2 / 3}$ \\
radiation/relativistic matter & $\frac{1}{3}$ & $\propto a^{-4}$ & $t^{1 / 2}$ \\
cosmological constant & -1 & $\propto a$ & $\exp H t$ \\
\hline \hline
\end{tabular}

Table 1.1: Scaling with the expansion factor of the constituents of an FLRW Universe and of the expansion factor with time for different matter components.

Let us denote by an upper index "(0)" the present-day values of the densities component; the scaling in terms of the redshift of the different energy densities is

$$
\Omega_{m}=\Omega_{m}^{(0)}(1+z)^{3}, \quad \Omega_{k}=\Omega_{k}^{(0)}(1+z)^{2}, \quad \Omega_{\Lambda}=\Omega_{\Lambda}^{(0)}
$$

and from the eq. (1.21) we obtain

$$
E(z) \equiv \frac{H(z)}{H_{0}}=\sqrt{\Omega_{m}^{(0)}(1+z)^{3}+\Omega_{k}^{(0)}(1+z)^{2}+\Omega_{\Lambda}^{(0)}} .
$$

that expresses the evolution of the Hubble function.

\subsubsection{The $\Lambda$ Cold Dark Matter Cosmological Model}

During the last four decades, the increased number and precision of astronomical observations allowed cosmologists to propose a standard model for the evolution of the Universe, known as concordance model. The Wilkinson Microwave Anisotropy Probe (WMAP) by NASA ${ }^{2}$ from 2003 to 2012, and more recently the PLANCK Satellite ${ }^{3}$ since 2013 have provided the necessary data to constraint models with unprecedented accuracy (Hinshaw et al. (2013), Planck Collaboration (2013i,j,l,m)); see Fig. 1.1.

In this model, the present period of accelerated expansion is due to a cosmological constant that provides a energy density of $\Omega_{\Lambda}^{(0)}=0.686 \pm 0.020$ in units of the critical density. The effect of the cosmological constant is that of a perfect fluid with an equation of state parameter $w=-1$. The second largest component is dark matter, needed to explain the dynamics of galaxies and the emergence of LSS. Its energy density is $\Omega_{D M}^{(0)}=0.314 \pm 0.020$. Finally, the present baryon fraction is $\Omega_{b}^{(0)} h^{2}=$

\footnotetext{
${ }^{2}$ http : //lambda.gsfc.nasa.gov

${ }^{3}$ http://sci.esa.int/jump.cfm?oid $=51557$
} 


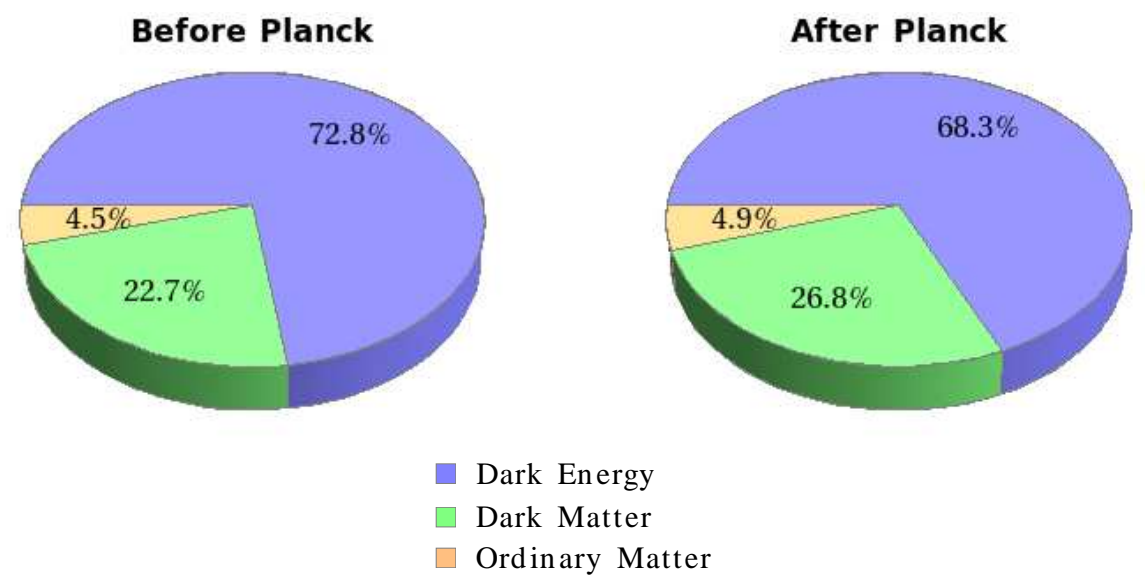

Figure 1.1: Energy density contents of the Universe, before and after the PLANCK 2013 results.

$0.02207 \pm 0.00033$ (Planck Collaboration, 2013j), making the Universe spatially flat. Indeed the constraint on the curvature of the Universe is $\Omega_{k}=-0.037_{-0.042}^{+0.044}$ (Hinshaw et al., 2013), validating the $\Lambda \mathrm{CDM}$ model.

In Fig. 1.2 we represent the evolution of the background energy densities of three different components, particularized for the $\Lambda \mathrm{CDM}$ model. There are three different cosmological eras: radiation dominated at high redshifts, matter dominated at intermediate redshifts, followed by the present period of accelerated expansion due to a cosmological constant.

If the recent BICEP2 measurement of B-mode polarization of the CMB with a tensor to scalar ratio of $r=0.2_{-0.05}^{+0.07}$ are an indication of primordial GWS generated during the period of inflationary expansion, then the cosmological model will be further vindicated since it would be the first direct probe of inflation as the source of tensor (and also scalar) perturbations. Nevertheless, one has to acknowledge that this value is in tension with the upper limits from WMAP ( $r<0.13$ at 95\% CL) and Planck $(\mathrm{r}<0.11$ at $95 \% \mathrm{CL})$ (BICEP2 Collaboration, 2014). The tension is very significant, with probability less than $\sim 0.1 \%$ if the observed deficit of large-scale temperature power is considered (Smith et al., 2014), but it is less so when the uncertainties in the dust polarization contribution are taken into account (Mortonson and Seljak, 2014).

\subsection{Cosmological Datasets to probe $\Lambda$ CDM model}

Observations of the distribution of galaxies, high red-shift SNe, gravitational lensing, and redshift distortions, have provided important complementary information that helped to brake some degeneracies on the determination of parameters from CMB data. Particular interesting were the results from the power spectrum of density perturbations from the 2dF survey of galaxies (Percival et al., 2001, Pope et al., 2004), 


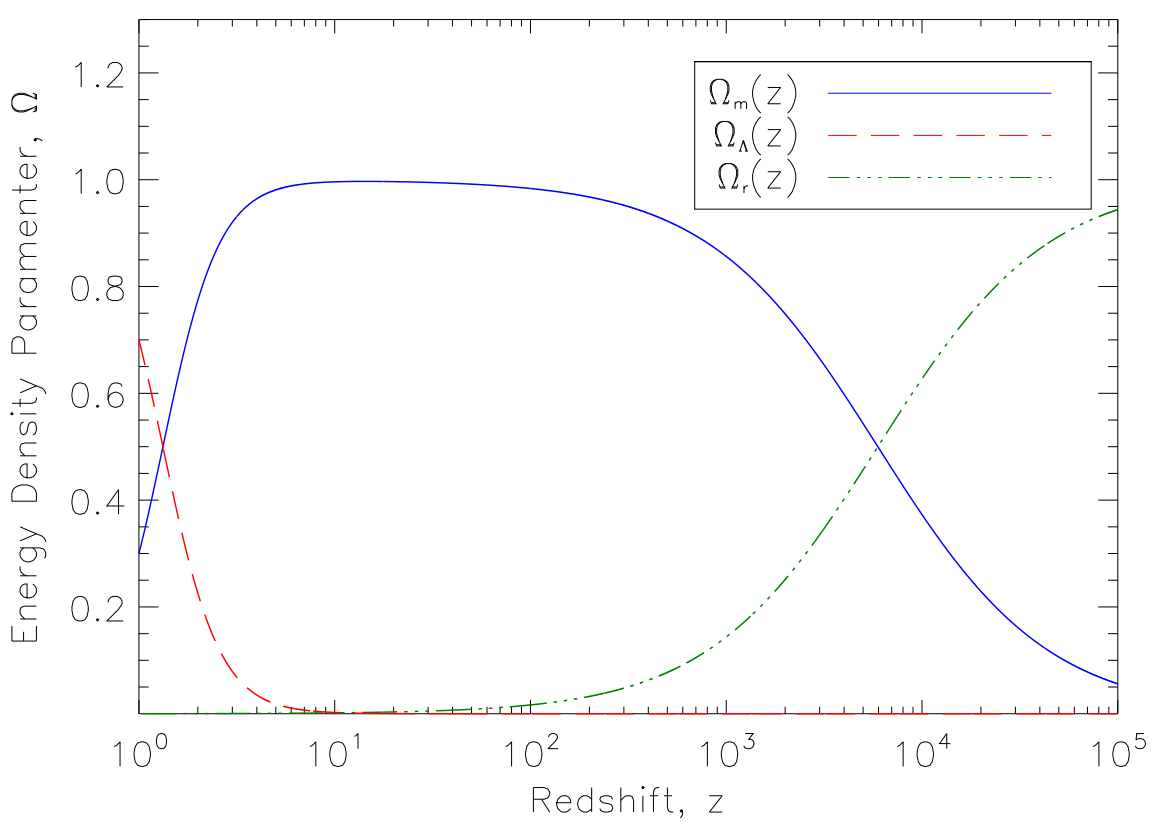

Figure 1.2: Evolution of the energy components, expressed in units of the critical density: radiation $\Omega_{r}$ (green, three dotted-dashed line), matter $\Omega_{m}$ (blue, solid line) and dark energy cosmological constant $\Omega_{\Lambda}$. At different redshifts, the energy budget is dominated by a different component, giving rise to the various cosmological eras.

the SDSS of galaxies (Tegmark et al., 2004). Luminosity distances from high redshift SNeIa allow us to constrain the expansion of the Universe up to redshift $z \sim=1.8$ (Riess et al. (2004), Astier et al. (2006), Clocchiati (2006), Suzuki et al. (2012)). In this section we will describe different cosmological observables that can be measured using astronomical data.

\subsubsection{The cosmic distances}

Distance measurements between astronomical sources at cosmological scales provided both the first observations of the expansion of the Universe and of its present acceleration. In an expanding Universe, the definition of distance is not unique and depends of the observational technique used to determine it. The following are the most important ones used in Cosmology:

Co-moving distance : distance between two observers co-moving with the Hubble flow; it is independent of time. If a photon emitted at time $t=t_{1}$ from a source at the radial coordinate $r=r_{1}$, is detected by an observer at time $t=t_{0}$ at $r=0$ the co-moving distance is

$$
d_{c} \equiv r_{1}=\int_{r_{1}}^{0} d r=-\int_{t_{1}}^{t_{0}} \frac{c}{a(t)} d t
$$


Using the eqs. (1.15), (1.23) and (1.24), ones can write

$$
d_{c}=\frac{c}{a_{0} H_{0}} \int_{0}^{z} \frac{d z^{\prime}}{E\left(z^{\prime}\right)},
$$

where $E(z)$ is given in eq. (1.31). If $z \ll 1$ one could expand in Taylor series the term $\frac{1}{E(z)}$, and then integrate eq. (1.33) to obtain

$$
c \simeq\left(a_{0} H_{0}\right) d_{c},
$$

that is the Hubble's law is verified at the low-redshift if the distance is the comoving distance, while if $z \gtrsim 1$ the higher-order terms become important.

Angular diameter distance : distance of an object of size $\Delta x$ at redshift $z$ subtending angular size $\Delta \theta$. The angular diameter distance can be written as

$$
d_{A}(z) \equiv \Delta x / \Delta \theta=a\left(t_{1}\right) \chi(r),
$$

where $t_{1}$ is the epoch the radiation was emitted and

$$
d_{A}(z)=\frac{1}{1+z} \begin{cases}\frac{d_{H}}{\sqrt{\Omega_{k}}} \sinh \left(\sqrt{\Omega_{k}} d_{c}(z) / d_{H}\right) & \text { for } \Omega_{k}>0, \\ d_{c}(z) & \text { for } \Omega_{k}=0, \\ \frac{d_{H}}{\sqrt{\left|\Omega_{k}\right|}} \sin \left(\sqrt{\left|\Omega_{k}\right|} d_{c}(z) / d_{H}\right) & \text { for } \Omega_{k}<0,\end{cases}
$$

where $d_{H} \equiv \frac{c}{H_{0}}$.

Luminosity distance : distance of an object derived from the intrinsic luminosity $L$ of the object whose measured flux is $\mathcal{F}$. Since the radiation emitted by the source is distributed over a surface $S=4 \pi\left(a_{0} \chi(r)\right)^{2}$ then, the luminosity distance

$$
d_{L}(z)=\sqrt{\frac{L}{4 \pi \mathcal{F}}} .
$$

can be written as

$$
d_{L}^{2}=\left(a_{0} \chi(r)\right)^{2} \frac{L_{s}}{L_{0}} .
$$

where the ratio ${ }^{4}$ is given by $\frac{L_{s}}{L_{0}} \equiv(1+z)^{2}$, and it is related to the angular diameter distance as

$$
d_{L}=a_{0} \chi(r)(1+z)=(1+z)^{2} d_{A} .
$$

\footnotetext{
${ }^{4}$ Let us write the energy of photons, emitted within a time-interval $\Delta t_{1}$, as $\Delta E_{1}$. The intrinsic luminosity is given by $L_{s}=\Delta E_{1} / \Delta t_{1}$. Similarly, for the observed one, it is $L_{0}=\Delta E_{0} / \Delta t_{0}$. Since the energy of a photon is inversely proportional to its wavelength $\lambda$, then $\Delta E_{1} / \Delta E_{0}=\lambda_{0} / \lambda_{1}=1+z$. Due to the constancy of the speed of light $c=\lambda / \Delta t$, then $\lambda 1 / \Delta t 1=\lambda 0 / \Delta t_{0}$, where $\lambda_{1}$ and $\lambda_{0}$ are, respectively, the wavelength of the radiation at the emitter and the observer and

$$
\frac{L_{s}}{L_{0}}=\frac{\Delta E_{1}}{\Delta E_{0}} \frac{\Delta t_{0}}{\Delta t_{1}}=(1+z)^{2} \text {. }
$$
}




\subsubsection{The Type Ia Supernovae}

Standard candles are sources whose intrinsic luminosity can be derived from other observations. In Cosmology, SNs are the most useful candles since they can be measured at large distances. For SN type Ia, their intrinsic luminosity can be derived from their luminosity curve. Observationally these objects are identified from their spectrum, that contains absorption lines of single ionized silicon without hydrogen lines. Their physical properties support the idea that they are the result of the collapse of a White Dwarf growing matter from a nearby companion when its mass exceeds the Chandrasekhar limit $\left(\sim 1.44 M_{\odot}\right)$ (Chandrasekhar, 1931). These explosions are among the most energetic and brightest in the Universe. Their peak luminosity correlates with the decrement in magnitude 15 days after their maximum brightness, $\Delta m_{15}$ (Hamuy et al., 1996). The absolute magnitude is

$$
M_{B} \simeq 0.8\left(\Delta m_{15}-1.1\right)-19.5
$$

and the distance modulus $\mu$ can be computed

$$
m-M \equiv \mu=5 \log d_{L}-5 .
$$

In 1998, Perlmutter et al. (1999), using 42 high-redshift SN Ia at redshifts [0.18, 0.83], and 18 low-redshift SN Ia of the Calan/Tololo Supernova Survey of Hamuy et al. (1996), showed that at the 99\% confidence level the Universe was being accelerated. During the last decade, the SuperNovae Legacy Survey (SNLS) (Astier et al., 2006), the ESSENCE survey (Davis et al., 2007) and the Hubble Space Telescope (HST) (Riess et al., 2004) have collected the largest sample of high redshift SN. The latest version 2.1 of the Union sample (Kowalski et al. (2008), Amanullah et al. (2010)) currently contains 833 SNe from 19 independent datasets (Suzuki et al., 2012). In Fig. 1.3 we represent luminosity distances and the Union 2.1 data $^{5}$. The solid red line corresponds to the $\Lambda \mathrm{CDM}$ model with Planck-2013 best fit cosmological parameters Planck Collaboration (2013j). Using these SNe data Suzuki et al. (2012) constrained the equation of state of dark energy for a flat Universe to $w=-0.985_{-0.077}^{+0.071}$ without detecting any significant change with redshift.

\subsubsection{The Cosmic Microwave Background radiation}

The CMB radiation was discovered by A.A. Penzias and R.W. Wilson in 1964. They measured an excess of temperature during the calibration process of a communication antenna. At $7 \mathrm{~cm}$ wavelength, the signal corresponded to a blackbody at 3.5 $\mathrm{K}$ of temperature isotropically distributed over the sky. Dicke et al. (1965) proposed that it was of a cosmological origin, indicating that at an early phase the Universe

\footnotetext{
${ }^{5}$ SuperNovae Cosmology Project web site: http://supernova.lbl.gov/Union/ .
} 


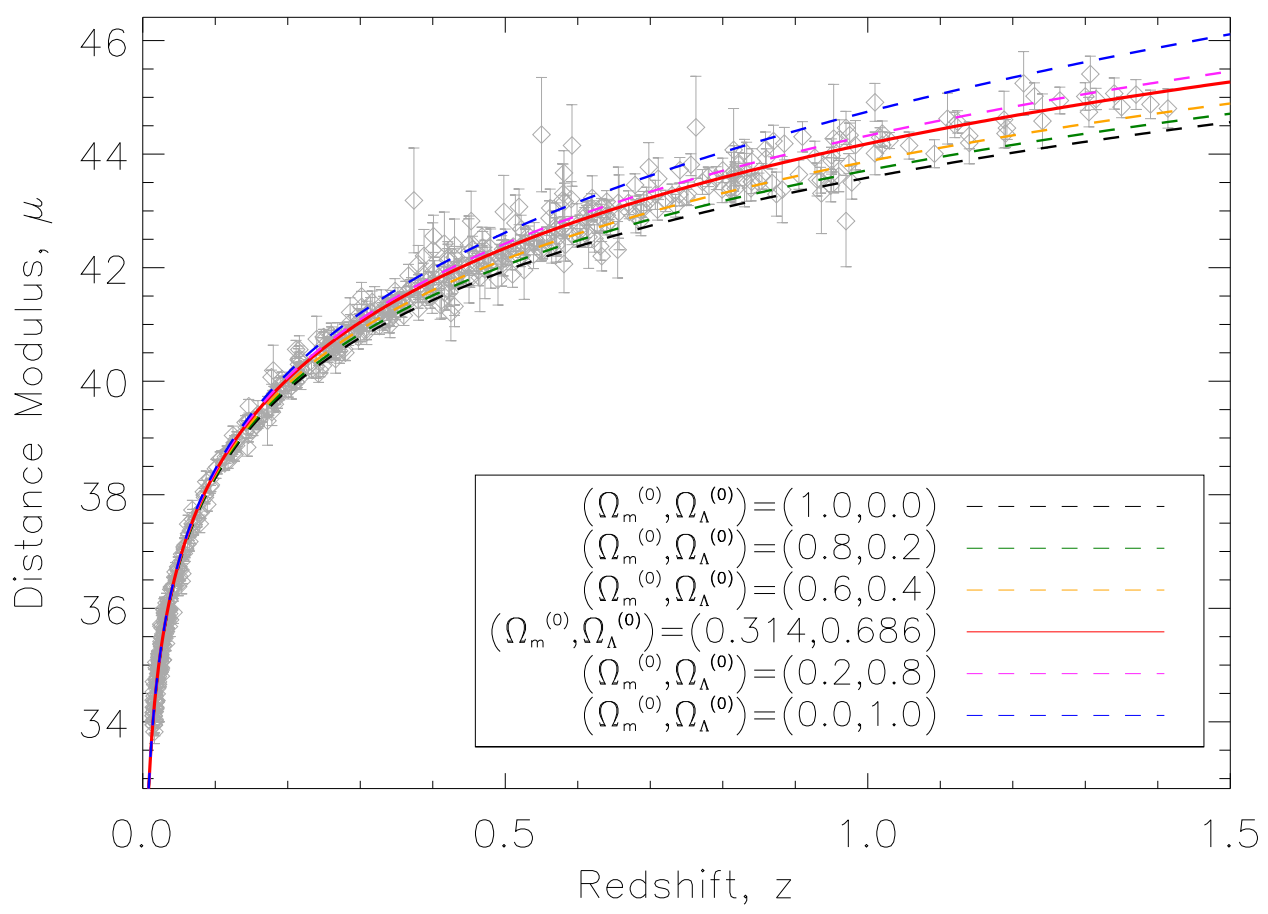

Figure 1.3: The SN Union 2.1 data set and some theoretical luminosity distances for different cosmological parameters of the concordance $\Lambda \mathrm{CDM}$ model. The bold solid line corresponds to parameters that best fit Planck data Planck Collaboration (2013j).

had reached a very high temperature and density. The CMB radiation together with the primordial abundances of light elements (Steigman, 2006) are among the strongest observational evidences in favor of a Hot Big Bang paradigm.

In its early stages of evolution, the Universe had a very high temperature and baryons and radiation were tightly coupled by means of Thomson scattering. After $t \sim 3 \times 10^{5}$ years, at $z \sim 1000$, the temperature had fallen below $\sim 3000 \mathrm{~K}$ and protons and electrons combined to form neutral hydrogen and other light elements such as ${ }^{3} \mathrm{He}$, ${ }^{4} \mathrm{He},{ }^{7} \mathrm{Li}$, during a period known as recombination. In the process, the opacity of the primordial plasma decreased and the photon mean free path exceeded the size of the horizon, decoupling radiation from baryons. Today, this flux of "primordial" photons constitutes the CMB radiation. It is an isotropic blackbody with anisotropies at the $10^{-5}$ level. The isotropy reflects the accuracy of the Cosmological Principle. The blackbody spectrum indicates that in the early Universe baryons and photons were in thermal equilibrium thanks to thermal bremsstrahlung and radiative Compton effects. If the expansion of the Universe is adiabatic, $P V^{\gamma}=$ const, with $\gamma=4 / 3$, the CMB temperature scales as

$$
T(z)=T_{0}(1+z)
$$


Since $\lambda=\lambda_{0}(1+z)^{-1}$, the black body spectrum was preserved by the expansion

$$
\exp \left(\frac{h c}{k_{B} \lambda T}\right) \simeq \exp \left(\frac{h c}{k_{B} \lambda_{0} T_{0}}\right)
$$

It was definitively measured by Fixsen et al. (1996) using the COBE-FIRAS observations.

\section{The Cosmic Microwave Background radiation anisotropies}

Although the CMB radiation field is highly isotropic it also shows well measured small departures from isotropy. These anisotropies are among the most informative data on the early stages of the evolution of the Universe. The first anisotropy detected was the dipole, induced by the motion of Local Group (LG) with respect to the CMB radiation. The Doppler effect due to our motion produces an anisotropy of amplitude $\frac{\Delta T}{T} \sim 10^{-3}$ depending on the angle $\theta$ between the direction of our motion with respect to line of sight

$$
\frac{\Delta T}{T}=\frac{v}{c} \cos \theta+\frac{v^{2}}{2 c^{2}} \cos 2 \theta+O\left(\frac{v}{c}\right),
$$

where $\theta$ represents the angle between the direction of the motion and line of sight, and $v$ is the velocity of LG. The term $\cos 2 \theta$ gives rise the quadrupole contribution. The velocity of the Solar System relatively to the CMB is $v \sim 370 \mathrm{~km} / \mathrm{s}$, and the corresponding dynamic quadrupole is $\sim 4 \mu \mathrm{K}$, smaller than its cosmological counterpart, of $\sim 15 \mu \mathrm{K}$ (Tegmark et al., 2003). Smaller scale anisotropies were generated by perturbations in the matter distribution. Their spectrum was predicted theoretically before being observed. Their amplitude is $\frac{\Delta T}{T} \sim 10^{-5}$. These fluctuations would be a direct result of the inflationary period (Harrison (1970), Zeldovich (1972), Wright et al. (1996), Peacock (1999)).

Temperature anisotropies can be described in terms of spherical harmonic

$$
\frac{\Delta T}{T}(\theta, \phi)=\Sigma_{l m} a_{l m} Y_{l m}(\theta, \phi),
$$

with

$$
a_{l m}=\int d \theta d \phi Y_{l m}^{*}(\theta, \phi) \frac{\Delta T}{T}(\theta, \phi),
$$

being the coefficients of the multipole expansion. Approximately, multipoles correspond to angular scale as: $l \sim \pi / \theta$. In the simplest inflation models, these coefficients are Gaussian distributed and uncorrelated for different modes $(l, m)$. Then, anisotropies are fully described by the angular power spectrum

$$
\left\langle a_{l m}^{*} a_{l^{\prime} m^{\prime}}\right\rangle=\delta_{l l^{\prime}} \delta_{m m^{\prime}} C_{l} .
$$

The power spectrum is related to the two point correlation function

$$
C(\theta)=\left\langle\frac{\Delta T}{T}(\hat{n}) \frac{\Delta T}{T}\left(\hat{n}^{\prime}\right)\right\rangle=\frac{1}{4 \pi} \Sigma_{l}(2 l+1) C_{l} P_{l}(\cos \theta),
$$


where $\hat{n}$ and $\hat{n}^{\prime}$ are the direction vectors of two points in the sky separated by an angle $\theta ; P_{l}$ are the Legendre polynomial of order $l$. Higher order multipole moments can be defined and computed. A non-vanishing three-point correlation function would measure the degree of non-Gaussianity of the CMB. Its Fourier transform is called bisprectrum and is an important observable to constrain the different variants of inflation.

All physical parameters describing the evolution of Universe determine the shape of the radiation power spectrum such as the height and location of the different acoustic peaks. From the analysis of the data at different angular scales, cosmological parameters can be derived. Unfortunately, the same power spectrum can be reproduced by different combinations of the cosmological parameters (degeneracies), so complementary datasets are needed (Hinshaw et al. (2013), Planck Collaboration (2013j)). In Fig. 1.4 we represent the radiation power spectrum defined as $\mathscr{D}_{l}=\frac{2 l(l+1) \mathcal{C}_{l}}{2 \pi}$ and its variation with cosmological parameters. In panel (a) we show the effect of varying the Hubble constant $H_{0}$ in the range $[60 \div 75]$. In (b) we represented the effect of changing the baryon fraction in the range $[0.01 \div 0.03]$. The effect of varying the dark matter density in the range $[0.1 \div 0.3]$ is presented in panel $(\mathrm{c})$. Finally, in $(\mathrm{d})$ we show the effect of the energy density associated with the curvature in the range $[0.1 \div 0.5]$. As comparison, in all panels the $\Lambda \mathrm{CDM}$ model is represented by a thick red line.

\section{CMB satellites: COBE, WMAP, and Planck}

From the discovery of the CMB radiation, many experiments were designed to measure its anisotropies and polarization modes. They include ground-based antennas, balloons, and satellites. The COsmic Background Explorer (COBE) measured the blackbody spectrum of the CMB radiation with unprecedented precision and detected anisotropies at large angular scales $\left(\theta \geq 10^{\circ}\right)$. These discoveries led John Mather and George Smoot, COBE's principal investigators, to receive the Physics Nobel Prize in 2006. The Wilkinson Microwave Anisotropy Probe (WMAP) and Planck satellites have produced maps of CMB temperature fluctuations with increasing resolution, lower noise levels and higher frequency coverage, measuring the cosmological parameters with improved accuracy.

COBE, launched in 1989, was composed of three different instruments: the Diffuse Infrared Background Experiment (DIRBE) to measure the cosmic infrared background (CIB) radiation, the Differential Microwave Radiometer (DMR) designed to measure temperature anisotropies and the Far Infrared Absolute Spectrophotometer (FIRAS) to determine the shape of the black body spectrum. DIRBE constrained models of the cosmological history of star formation and dust and heavy element production in the early Universe. DMR detected intrinsic anisotropies at a level of $10^{-5}$ for the first time. 


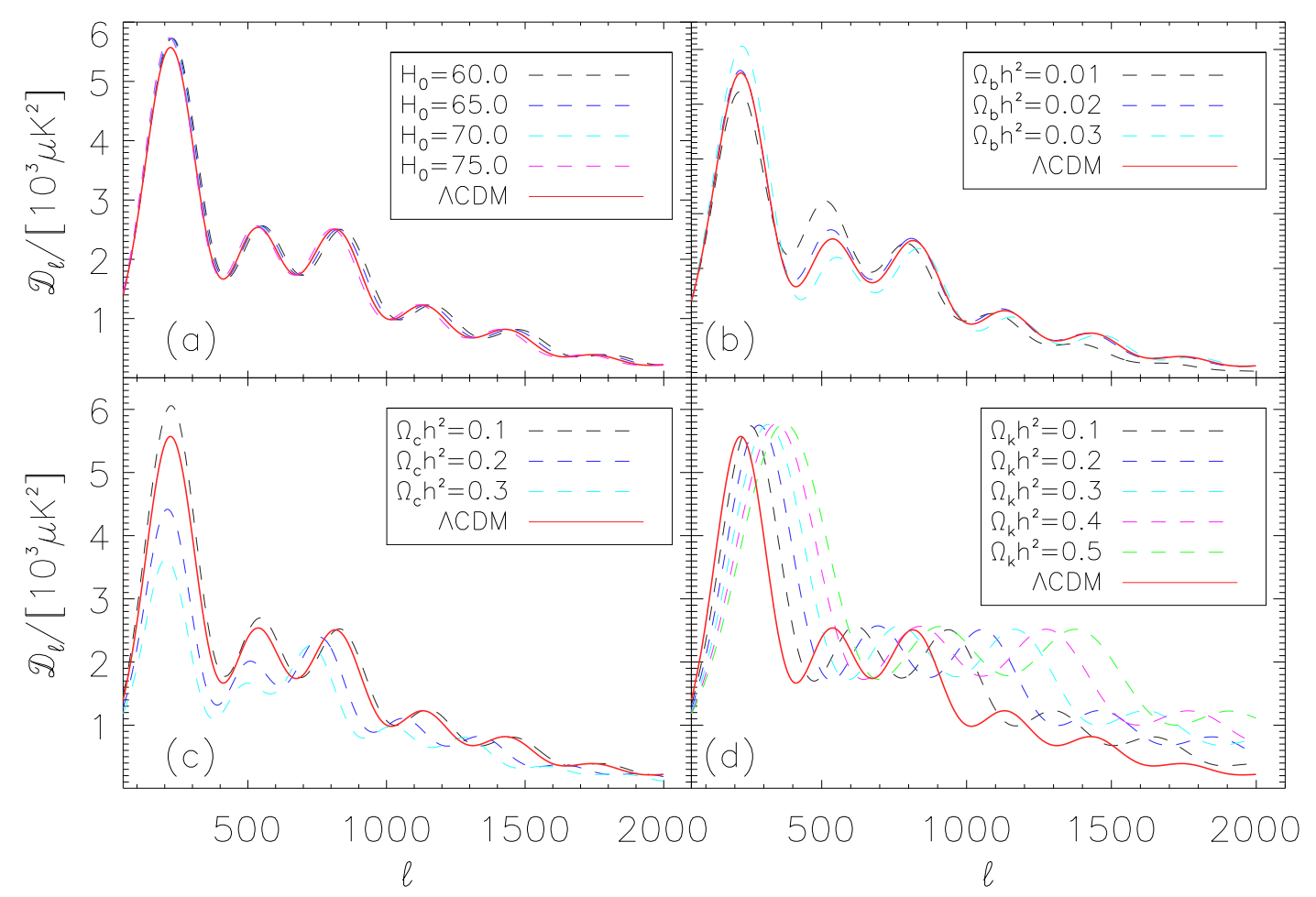

Figure 1.4: Variation of the acoustic peaks of the CMB radiation power spectrum with cosmological parameters as function of the multipole $l$. From (a) to (d) we show the effect of the Hubble constant $H_{0}$, baryon density, dark matter density and curvature, respectively. The red solid line corresponds to the $\Lambda \mathrm{CDM}$ model.

Finally, FIRAS detected the blackbody spectrum with a temperature of $2.725 \pm 0.002$ $\mathrm{K}$ and constrained deviations of this spectrum to be smaller than one part in $10^{5}$.

In 2001, the WMAP satellite was launched to measure the power spectrum of CMB temperature anisotropies with better precision than COBE, to determine the energy and matters content of the Universe and its geometry and test the Big Bang model and the cosmic inflation theory (Hinshaw et al., 2013). The experiment was designed to have uncorrelated pixel noise and to minimize the systematic errors. Its sensitivity was 45 times better than that of COBE and its angular resolution 33 times higher. In Table 1.2 we summarize the mission characteristics ${ }^{6}$.

WMAP have observed the sky in five different frequencies channels, measuring the foreground contaminations due to the Milky Way and extra-galactic sources. The lower frequencies are dominated by synchrotron radiation and free-free emission, while the higher frequencies are dominated by dust. Their spectral properties allowed us to identify and quantify the amount of foreground contamination, and hence to remove it (Bennett et al., 2013). Its measurements have favored the $\Lambda$ CDM model, shown to fit very well the power spectrum (Hinshaw et al., 2013).

\footnotetext{
${ }^{6}$ http : //lambda.gsfc.nasa.gov/product/map/current/
} 


\begin{tabular}{|lccccc|}
\hline \hline & K-Band & Ka-Band & Q-Band & V-Band & W-Band \\
\hline \hline Wavelength $(\mathrm{mm})$ & 13 & 9.1 & 7.3 & 4.9 & 3.2 \\
Frequency $(\mathrm{GHz})$ & 23 & 33 & 41 & 61 & 94 \\
Bandwidth $(\mathrm{GHz})$ & 5.5 & 7.0 & 8.3 & 14.0 & 20.5 \\
Beam Size $(\mathrm{deg})$ & 0.88 & 0.66 & 0.51 & 0.35 & 0.22 \\
\hline \hline
\end{tabular}

Table 1.2: WMAP Mission characteristics.

In May 2009 the Planck satellite was launched and in April 2013 the Collaboration released their first all-sky maps. The data covered a frequency range from 30 to $857 \mathrm{GHz}$; the frequency response of the different detectors is well approximated by a Gaussian (Planck Collaboration, 2013a). The 30, 44 and $70 \mathrm{GHz}$ channels correspond to the Low Frequency Instrument (LFI). The data has an angular resolution of 32', 27' and 13', respectively (Planck Collaboration $(2013 b, c)$ ). The higher frequencies, from 100 to $845 \mathrm{GHz}$ correspond to the High Frequency Instrument (HFI) and the data had resolutions from 9.66 to 4.63 arcminutes (Planck Collaboration, 2013d). The technical characteristics of the mission are given in Table 1.3. More detail can be found in the Planck Legacy Archive?

\begin{tabular}{|lccccccccc|}
\hline \hline Frequency Channels $(\mathrm{GHz})$ & 30 & 44 & 70 & 100 & 143 & 217 & 353 & 545 & 857 \\
\hline \hline Wavelength $\lambda_{0}(\mathrm{~mm})$ & 10.0 & 6.82 & 4.29 & 3.0 & 2.1 & 1.4 & 0.85 & 0.55 & 0.35 \\
Frequency $\nu_{0}(\mathrm{GHz})$ & 28.4 & 44.1 & 70.4 & 100 & 143 & 217 & 353 & 545 & 857 \\
Bandwidth $\Delta \nu(\mathrm{GHz})$ & 6 & 8.8 & 14 & 33 & 47 & 72 & 116 & 180 & 283 \\
Beam FWHM $(')$ & 32.65 & 27.00 & 13.01 & 9.94 & 7.04 & 4.66 & 4.41 & 4.47 & 4.23 \\
Noise $/$ pix $\left(\sigma_{\text {noise }} / \mu \mathrm{K}\right)$ & 51 & 52 & 15 & 12 & 19 & 58 & - & - & - \\
\hline \hline
\end{tabular}

Table 1.3: Technical characteristics of Planck channels.

Together with the intrinsic (cosmological) CMB signal, the data contained secondary anisotropies due to the SZ effect and foreground contributions like galactic and point source emission and instrumental noise (Planck Collaboration, 2013a). At LFI frequencies synchrotron and free-free emission are the dominant foregrounds while dust and molecular CO lines emission are dominant in the HFI range (Planck Collaboration, 2013f). All those contributions have to be removed prior to analyze the CMB radiation power spectrum (Planck Collaboration, 2013i), Integrated Sachs-Wolfe effect (ISW) (Planck Collaboration, 2013h), gravitational lensing (Planck Collaboration, 2013k), primordial non-Gaussianity (Planck Collaboration, 2013o) and the TSZ and KSZ components (Planck Collaboration, 2012). To this purpose, component separation methods have been applied to the data (Planck Collaboration, 2013f). As an illustration, in Fig. 1.5 we show two 10-square-degree patches corresponding to WMAP

\footnotetext{
${ }^{7}$ http : //www.sciops.esa.int/wikiSI/planckpla/index.php?title = Main $\mathrm{P}$ age
} 
and Planck data on the same region. The Figure illustrates how Planck higher angular resolution allows to resolve more structure than WMAP.

ILC WMAP map

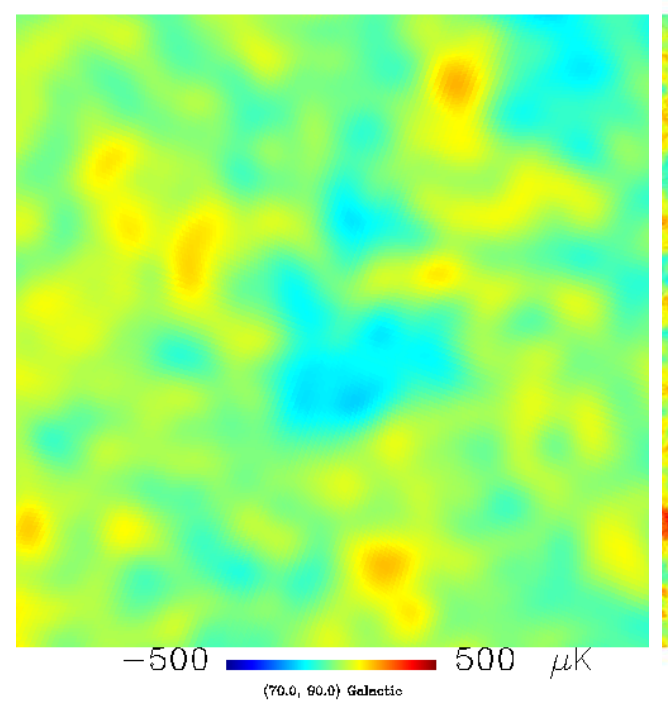

Planck SMICA map

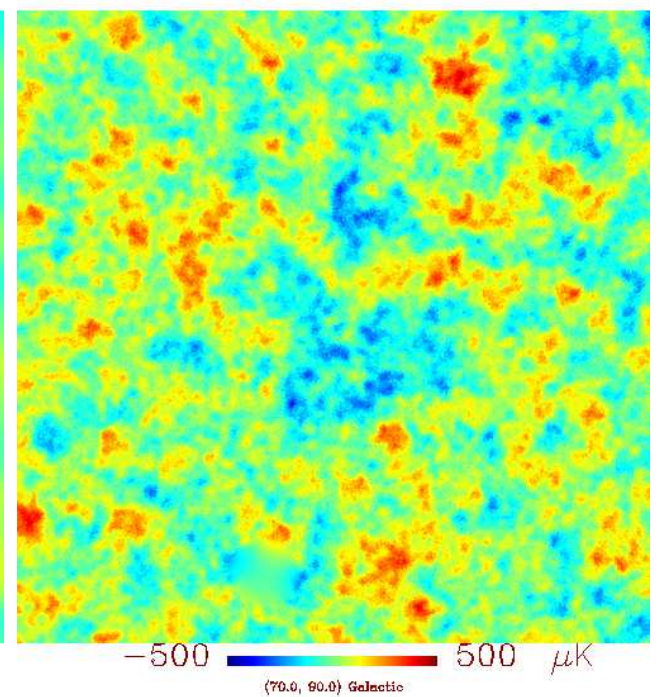

Figure 1.5: $500 \times 500$ screen pixels, with a resolution of $2 \operatorname{arcmin} /$ screen pixel at the center as seen by the $\mathrm{CMB}$ radiation satellites: WMAP and Planck. The temperature range is $[-500,500] \mu \mathrm{K}$. The images were taken from the foreground clean Internal Linear Combination (ILC) and the SMICA maps by WMAP and Planck, respectively.

Comparing WMAP and Planck results, Planck data prefers a flat $\Lambda$ CDM model with a lower value of the Hubble constant and higher matter content than WMAP Planck Collaboration (2013j). Table 1.4 summarizes the results on cosmological parameters obtained by WMAP 9 yrs data and Planck.

\begin{tabular}{|lccc|}
\hline \hline Parameter & WMAP & Planck & Planck+WMAP \\
\hline \hline Age of the Universe $\left(t_{0} / \mathrm{Gyrs}\right)$ & $13.74 \pm 0.11$ & $13.813 \pm 0.048$ & $13.817 \pm 0.048$ \\
Hubble's constant $\left(H_{0} / \mathrm{km}\right.$ & $\mathrm{Mpcs}$ & & \\
Baryon density $\left(\Omega_{b} h^{2}\right)$ & $70.0 \pm 2.2$ & $67.4 \pm 1.4$ & $67.3 \pm 1.2$ \\
Cold DM density $\left(\Omega_{c} h^{2}\right)$ & $0.02264 \pm 0.00050$ & $0.02207 \pm 0.00033$ & $0.02205 \pm 0.00028$ \\
DE density $\left(\Omega_{\Lambda}\right)$ & $0.7138 \pm 0.0045$ & $0.1196 \pm 0.0031$ & $0.1199 \pm 0.0027$ \\
Density fluctuations $\left(\sigma_{8}\right)$ & $0.821 \pm 0.025$ & $0.686 \pm 0.020$ & $0.686_{-0.016}^{+0.018}$ \\
Scalar spectral index $\left(n_{s}\right)$ & $0.972 \pm 0.013$ & $0.834 \pm 0.027$ & $0.829 \pm 0.012$ \\
Reionization optical depth $(\tau)$ & $0.089 \pm 0.014$ & $0.0916 \pm 0.0094$ & $0.9603 \pm 0.0073$ \\
Curvature $\left(\Omega_{k}\right)$ & $-0.037_{-0.042}^{+0.044}$ & - & $0.089_{-0.014}^{+0.012}$ \\
\hline \hline
\end{tabular}

Table 1.4: Best-fit cosmological parameters from WMAP nine year data and Planck 2013 first Data Release (Bennett et al. (2013), Planck Collaboration (2013j)).

The Planck measurement of the Hubble constant is consistent within $1 \sigma$ with the measurement provided by WMAP 9yr. However, the Planck best-fit model requires a Hubble constant that is significantly lower than expected from traditional measurement techniques raising the possibility of systematic errors in the latter. $H_{0}$ measurements 
provided by SNeIa plus Cepheids disagree with Planck at the $2.5 \sigma$ level (Planck Collaboration, 2013j). This tension, if not alleviated with future data, could reveal new physics, such as a time-varying dark energy equation of state, additional relativistic particles, or neutrino masses.

\subsection{Shortcomings of the concordance $\Lambda$ CDM model}

The correct theory of gravity has to match observations at all scales, from astrophysical to cosmological, from planetary dynamics, to collapsed objects to the large scale structure. It must reproduce Newtonian dynamics in the weak-energy limit and at small velocities $(v \ll c)$. Solar System tests are the first observational step because they are experimentally well measured (Will, 2006). Then, galactic dynamics has to be reproduced taking in to account the baryonic constituents, directly from the observations of stars, sub-luminous components as planets, dwarf stars, dust and gas. Finally, it has to explain the emergence of LSS in the Universe, the cosmological expansion rate, the density parameters, age, etc (Peebles (1980), Peacock (1999)). The simplest theory that satisfies the above requirements is the GR and the concordance cosmological model is its translation to Cosmology. Due to its simplicity and capacity to explain large datasets, the $\Lambda \mathrm{CDM}$ model is considered the standard model in Cosmology. Nevertheless, it requires two unknown components to fit the observations: DM and DE. Even many candidates have been proposed, no clear identification has been found yet (Bergstrom (2009), Peter (2012)).

The photometric and spectroscopic mass estimates of luminous matter of selfgravitating systems such as stellar clusters, galaxies, groups and clusters of galaxies show an important deficit compared with their dynamical mass. Initially, the problem of missing mass was solved by adding a matter component that did not interact with radiation (hence the name "dark"). Early astronomical candidates were MAssive Compact Halo Objects (MACHOs) and ReAlly Massive Baryon Objects (RAMBOs), sub-luminous compact objects (or clusters of objects) like BHs and NSs that could not have been observed due to several selection effects. At the particle level, it was proposed that Weakly Interacting Massive Particles (WIMPs) low massive particles that interact weakly with ordinary matter could make most of the missing mass in the Universe. In either case there are no experimental evidences of their existence.

With respect to the other main parameter of the $\Lambda$ CDM model our understanding requires a fine tuning of the initial conditions of many orders of magnitude. For instance, the energy density associated to the present period of acceleration is

$$
\rho_{\Lambda} \approx \frac{\Lambda}{8 \pi G} \approx 10^{-47} \mathrm{GeV}^{4}
$$

In particle physics, the cosmological constant is associated with vacuum fluctuations 
of the zero energy level of an empty space. At the Planck scale,

$$
\rho_{v a c} \simeq 10^{76} \mathrm{GeV}^{4},
$$

that would be 123 orders of magnitude greater than the observed value. For this reason, the Cosmological Constant is seen as a particular version of a more general energy density known as Dark Energy (DE). Alternative sources of the present period of accelerated expansion are quintessence, Chapligyn gas, phantom, etc, that have their origin in string theory, brane cosmology, the holographic principle applied to a cosmological setting, etc (Peebles and Ratra (2003), Tsujikawa (2011)). In order to give rise to a period of acceleration, these fluids are required to have $\omega<-1 / 3$. But this opens another fine tune problem, the coincidence problem. Matter and dark energy evolve very differently with redshift but today have a similar value which implies that their ratio at Planck scales would differ by 70 orders of magnitude.

At the theoretical level, we are still lacking a quantum theory of gravity. The main problem is that in GR the space-time cannot be fixed as scenario where the phenomena are studied like in electromagnetic theory. Here, the space-time is itself a dynamical variable that is obtained by solving the equations of motion. Currently, two approaches have been used to study the quantization of gravity: a covariant (loop quantum gravity) and a perturbative (string theory) approach, but a final theory of Quantum Gravity is still out of sight. To make progress, it is necessary to identify the dark candidates at the particle level and understand their properties.

Alternatively to the shortcomings represented by the DM and DE, one can try to preserve the good results of GR but avoiding the introduction of new ingredients in the cosmic energy density budget. This approach leads to new theories of gravity generally called Extended Theories of Gravity. Below we will review the main features of these theories, concentrating on their testable aspects in order to analyze if they represent an alternative and a viable description of the gravitational interaction.

\subsection{Extended Theories of Gravity}

Extended Theories of Gravity (ETGs) are extensions of GR obtained by including in the Lagrangian higher-order curvature invariants (such as $R^{2}, R_{\mu \nu} R^{\mu \nu}, R^{\mu \nu \alpha \beta} R_{\mu \nu \alpha \beta}$, $R \square R$, or $R \square^{k} R$ ) and minimally or non-minimally coupled terms between scalar fields and geometry (such as $\phi^{2} R$ ) (Capozziello and De Laurentis, 2011). From the theoretical point of view, there are no reasons to restrict the Lagrangian to be linear in the Ricci scalar and minimally coupled with matter. ETGs make very concrete predictions that differ from GR and therefore are testable even with current instruments. One important feature of the ETGs is that the conservation laws, derived from gauge invariance, are well defined only at low energy limit. As a consequence, the funda- 
mental physical constants can vary (Barrow and Ottewill (1983), Uzan (2003)). These theories are also important from a cosmological point of view, because they can directly explain the accelerated expansion of the Universe without requiring additional energy components, avoiding the fine tune problems on nature of the DE described above (Amendola and Euclid Theory Working Group, 2013).

ETGs can be classified in: (A) Scalar-Tensor Theories if the geometry is nonminimally coupled to some scalar field and (B) Higher Order Theories if the action contains derivatives of the metric components of order higher than two. Combinations of both types give rise higher order/scalar-tensor gravity theories. In the most general case the action can be written as

$$
\mathcal{S}=\int d^{4} x \sqrt{-g}\left[F\left(R, \square R, \square^{2} R, . . \square^{k} R, \phi\right)-\frac{\epsilon}{2} g^{\mu \nu} \phi_{; \mu} \phi_{; \nu}+2 \kappa \mathcal{L}^{(m)}\right],
$$

where $F$ represents a generic function of curvature invariants, and scalar field $\phi, \epsilon=$ $0, \pm 1$ specifies the nature of scalar field to be standard, phantom or no-dynamical (Faraoni (2005), Rubano and Scudellaro (2005), Nojiri et al. (2005)).

\section{6 $f(R)$ gravity: general formalism}

The approach described above is the most generic. We shall concentrate in $f(R)$ theories, that gives a general prescription to extend the GR theory of gravity in a simple manner. The extension is performed by replacing the Einstein-Hilbert Lagrangian, that is linear in the Ricci scalar, with a more general function of the curvature $f(R)$. In the metric formalism, one arrives at the field equations by taking variations with respect to the metric; the connections are not treated independently from the metric. The field equations are fourth order and admit larger families of solutions than standard GR. In these theories the action is

$$
S_{f(R)}=\int d^{4} x \sqrt{-g} f(R),
$$

and the variational principle $\delta S_{f(R)}=0$ gives

$$
\begin{aligned}
\delta \int d^{4} x \sqrt{-g} f(R)= & \int d^{4} x \sqrt{-g}\left[f^{\prime}(R) R_{\mu \nu}-\frac{1}{2} g_{\mu \nu} f(R)\right] \delta g^{\mu \nu}+ \\
& +\int d^{4} x \sqrt{-g} f^{\prime}(R) g^{\mu \nu} \delta R_{\mu \nu} .
\end{aligned}
$$

The prime denotes derivatives respect to $R$. In the local inertial frame

$$
\partial_{\alpha} g_{\mu \nu}=\nabla_{\alpha} g_{\mu \nu}=0
$$

and eq. (1.51) can be re-written as (Capozziello and De Laurentis, 2011)

$$
\begin{aligned}
& \delta \int d^{4} x \sqrt{-g} f(R)=\int d^{4} x \sqrt{-g}\left[f^{\prime}(R) R_{\mu \nu}-\frac{1}{2} f(R) g_{\mu \nu}\right] \delta g^{\mu \nu}+ \\
&+\int d^{4} x\left[g_{\mu \nu} \partial^{\sigma} \partial_{\sigma}\left(\sqrt{-g} f^{\prime}(R)\right)-g_{\sigma \nu} \partial^{\mu} \partial_{\sigma}\left(\sqrt{-g} f^{\prime}(R)\right)\right] \delta g^{\mu \nu},
\end{aligned}
$$


that leads to the fourth order vacuum field equations

$$
f^{\prime}(R) R_{\mu \nu}-\frac{f(R)}{2} g_{\mu \nu}=f^{\prime}(R)_{; \mu \nu}-g_{\mu \nu} \square f^{\prime}(R)
$$

that can be also written in the Einstein-like form

$$
f^{\prime}(R) R_{\mu \nu}-\frac{f^{\prime}(R)}{2} g_{\mu \nu} R+\frac{f^{\prime}(R)}{2} g_{\mu \nu} R-\frac{f(R)}{2} g_{\mu \nu}=f^{\prime}(R)_{; \mu \nu}-g_{\mu \nu} \square f^{\prime}(R),
$$

that leads to

$$
R_{\mu \nu}-\frac{1}{2} g_{\mu \nu} R+\Delta G_{\mu \nu}=0
$$

where $\Delta G_{\mu \nu}$, that denotes the terms coming from the extension of GR, is

$$
\Delta G_{\mu \nu}=\frac{1}{f^{\prime}(R)}\left\{f^{\prime}(R)_{; \mu \nu}-g_{\mu \nu} \square f^{\prime}(R)+g_{\mu \nu} \frac{\left[f(R)-f^{\prime}(R) R\right]}{2}\right\} .
$$

The trace of field equation (1.56) is given by

$$
f^{\prime}(R) R-2 f(R)+\Delta G=0
$$

where $\Delta G=f^{\prime}(R) \operatorname{Tr}\left\{\Delta G_{\mu \nu}\right\}$. The right side of the eq. (1.54) can be interpreted as an effective energy-momentum tensor that includes curvature effects due to the higher order term in the Lagrangian. This approach could be very useful for practice purposes, and in order to test ETGs on astrophysical and cosmological datasets, we need to rewrite the equations of field (1.54) in presence of a matter distribution. Let us generalize the action adding a minimally coupled matter contribution to the eq. (1.50)

$$
S=\int d^{4} x \sqrt{-g}\left[f(R)+\mathcal{X} \mathcal{L}_{m}\right]
$$

Here, $\mathcal{X}=\frac{8 \pi G}{c^{4}}$ is the usual coupling constant of gravitational field equations. Eq. (1.54) now reads

$$
f^{\prime}(R) R_{\mu \nu}-\frac{1}{2} f(R) g_{\mu \nu}-f^{\prime}(R)_{; \mu \nu}+g_{\mu \nu} \square f^{\prime}(R)=\mathcal{X} T_{\mu \nu}
$$

where $T_{\mu \nu}=\frac{-2}{\sqrt{-g}} \frac{\delta\left(\sqrt{-g} \mathcal{L}_{m}\right)}{\delta g^{\mu \nu}}$ is the energy momentum tensor of matter. The trace of the field equations is

$$
3 \square f^{\prime}(R)+f^{\prime}(R) R-2 f(R)=\mathcal{X} T,
$$

where $T$ is the trace of the energy-momentum tensor. Eqs. (1.60) and (1.61) will be used in our study of star formation and gravitational waves emission. 


\subsubsection{Formalisms and frames in $f(R)$ gravity}

The 4-dimensional action in $f(R)$ gravity represented by eq. (1.50) leads to the field equations (1.54) by varying the action with respect to the metric tensor $g_{\mu \nu}$. In this approach, called metric formalism, the affine connections are the Levi-Civita connections in eq. (1.4). In the Palatini formalism, the affine connections are seen as a variable, and they are independent by the metric tensor $g_{\mu \nu}$. Therefore, the action is varied independently with respect to the metric and the connections. The Palatini approach in GR in fully equivalent to the metric approach, because of the field equations the connections have to be the metric one, therefore there is no reason to prefer an approach to each other (Wald, 1984). Nevertheless, in ETGs, the two approaches lead to different field equations and different physics. Although the causal structure of the space time is defined by the metric tensor, the trajectories of particles are determined by the connections. The Palatini approach of $f(R)$-gravity is usually translated in the bi-metric approach where, instead of having a metric tensor and an independent connection, there are two metric component, $g_{\mu \nu}$ and $h_{\mu \nu}=f^{\prime}(R) g_{\mu \nu}$. The latter is directly related to the connections because they can be re-written as the common LeviCivita connection for $h_{\mu \nu}$ (de Felice and Tsujikawa (2010), Capozziello and Faraoni (2010), Olmo (2011)). One of the main advantages of using the Palatini approach is that it leads to second order field equations that are free from the instability due to the negative signs of $f^{\prime \prime}(R)$. Furthermore, the background cosmological dynamic has been investigated showing the possibility to obtain the sequences of radiation, matter, and accelerated epochs (see for details de Felice and Tsujikawa (2010) and references within). However, DE models based on Palatini formalism are not compatible either with the observations of large-scale structure and with Standard Model of particle physics because of the large coupling of DE and non-relativistic matter. Moreover, it has been pointed out that, in the Palatini approach, the Cauchy problem is not wellformulated as it is in the metric formalism. In the light of this considerations, we will restrict our study using the metric formalism although it leads to more complicated field equations.

Another point of discussion is the "Einstein versus Jordan frame" controversy. Both sets of variables are related to each other by a conformal transformation. It does not represent a change in a space time reference frame. The field equations (1.54) are expressed in the Jordan frame, and they can be translated in the Einstein frame under a conformal transformation. As a consequence, eqs. (1.54) are transformed into equations very similar to the GR ones, this fact led to the nomenclature "Einstein frame". Although it is always possible to transform back and forth from the Jordan and Einstein frames because of they are conformally equivalent, there are physical differences in the two frames when matter is taken into account. Photons follow null 
geodesics in both frames, but time-like geodesics in the Jordan frame do not transform into geodesics in the Einstein frames and the frames are not physically equivalent (Capozziello and Faraoni, 2010). Since the theoretical predictions are affected by the choice of the frame, the fundamental question is: what is the correct frame to describe the observations? Many authors argue the Jordan frame is the physical frame because it is easier to relate to observations but others prefer the Einstein frame on the basis of energy considerations (Magnano and Sokolowski (1994), Faraoni and Gunzig (1999)). In general, it is simpler to perform calculations in the Einstein frame, but it is more difficult to connect them with the observations. Therefore, many authors use the Einstein frame to solve the field equations and then they return to the Jordan frame to compare the theoretical prediction with astrophysical observations. However, this approach it is correct only if the two frames are physically equivalent, and the only way to make sure it is to compare the physics in the two conformal frames at the level of the Lagrangian, of the field equations, and of their solutions.

Since we are interested in testing ETGs using different astrophysical dataset, it was more convenient to express all equations and theoretical predictions in the Jordan frame.

\subsection{2 $f(R)$-models}

Starobinsky (1980) represented the first attempt to describe the acceleration of the Universe extending GR. Models such as $f(R)=R+\alpha R^{n}$, that include for $n=2$ the Starobinsky's model, gave rise quadratic corrections in the Ricci scalar that have been particularly relevant in cosmology since they allow to explain the early acceleration of the Universe. Afterwards, many models have been considered and tested. Some examples of models having viable cosmological solutions listed in Table 1.5. These models are also studied as viable $f(R)$-gravities models to address the Solar System tests and stochastic gravitational waves background (Capozziello et al., 2009b).

Instead to fix the Lagrangian, we shall study to $f(R)$ theories that are analytic function of the Ricci scalar that can be expanded in Taylor series

$$
f(R)=\sum_{n=0}^{+\infty} \frac{f^{n}\left(R_{0}\right)}{n !}\left(R-R_{0}\right)^{n}=f_{0}+f_{0}^{\prime} R+f_{0}^{\prime \prime} R^{2}+f_{0}^{\prime \prime \prime} R^{3}+\ldots
$$

where $f^{n}(R)=\left.\frac{d^{n} f(R)}{d R^{n}}\right|_{R=0}$. This approach is more general with respect fixing a particular form of the Lagrangian, indeed it will allow us to confirm or rule out large sets of models by using data. In analytic $f(R)$ models, the Cauchy problem is well posed only if $f_{0}^{\prime \prime}<0$. This condition guarantees the existence of stable cosmological solutions for perfect fluids (Capozziello and Vignolo, 2009). Every $f(R)$ theory can be be linearized producting massive modes of gravitational radiation (Capozziello et al., 


\begin{tabular}{|l|l|c|}
\hline \hline$f(R)$-model & Features & \multicolumn{1}{c|}{ Ref. } \\
\hline \hline$f_{0} R^{n}$ & $\begin{array}{l}\text { Fits Low surface brightness galaxy } \\
\text { rotation curves. }\end{array}$ & Capozziello et al. (2007) \\
\hline$R-m^{2} \frac{c_{1}\left(R / m^{2}\right)^{n}}{c_{2}\left(R / m^{2}\right)^{n}+1}$ & $\begin{array}{l}\text { Satisfies both cosmological and Solar } \\
\text { System tests in the small-field limit. }\end{array}$ & Hu and Sawicki (2007) \\
\hline$R+\lambda R_{c}\left(\frac{R}{R_{c}}\right)^{q}$ & $\begin{array}{l}\text { Satisfies both cosmological and Solar } \\
\text { System tests in the small-field limit. } \\
\text { Free from singularities. }\end{array}$ & Amendola and Tsujikawa (2008) \\
\hline$R+R_{*} \ln \left(1+\frac{R}{R_{*}}\right)$ & $\begin{array}{l}\text { Cosmological viable solutions; } \\
\text { distinguishable from } \Lambda \text { CDM; } \\
\text { compatible with relativistic stars; } \\
\text { free of singularities. }\end{array}$ & Miranda et al. (2009) \\
\hline$R+\lambda R_{c}\left[\left(1+\frac{R^{2}}{R_{c}^{2}}\right)^{-p}-1\right]$ & $\begin{array}{l}\text { Satisfies both cosmological and Solar } \\
\text { System tests in the small-field limit. }\end{array}$ & Starobinsky (2007) \\
\hline
\end{tabular}

Table 1.5: $f(R)$ models having viable cosmological solutions.

2008). In the model expressed by eq. (1.62), the graviton massive states can be defined as

$$
m_{g}=\left(-\frac{f_{0}^{\prime}}{3 f_{0}^{\prime \prime}}\right),
$$

and they are positively under the condition $f_{0}^{\prime \prime}<0$ discussed above. We will limit our study to models verifying this condition.

\subsubsection{The chameleon mechanism in $f(R)$ gravity}

To make compatible $f(R)$ models with local gravity constraints, these theories usually require a "chameleon mechanism". When considering theories with a nonminimally coupled scalar field, one has to impose strong conditions on the effective mass of the scalar field so the tight constraints on the Solar system are verified (Khoury and Weltman (2004), Khoury and Wyman (2009)). This is a peculiarity exclusive of $f(R)$ models that need to require $m r<<1$ where $m$ is the effective mass of the scalar degree of freedom of the theory, and $r$ is the scale. The effective mass $m$ depends on the space-time curvature or, alternatively, on the matter density distribution of the environment. The scalar degree of freedom can have a short range (i.e. $m>$ $10^{3} \mathrm{eV}$ correspond to a range $\lambda<0.2 \mathrm{~mm}$ ) at Solar System densities $(\rho \simeq 1-10$ $\mathrm{g} / \mathrm{cm}^{-3}$ ), escaping the experimental constraints, and have a long range at cosmological densities $\left(\rho_{c}^{(0)} \simeq 10^{-29} \mathrm{~g} / \mathrm{cm}^{-3}\right)$, and it can propagate freely affecting the cosmological dynamic, and driving the accelerated expansion (see for details de Felice and Tsujikawa (2010)). Similar mechanisms have been proposed in the literature from symmetron to braneworld models with the same purpose (Dvali et al. (2000), Nicolis et al. (2009), Hinterbichler and Khoury (2010)). 


\subsection{The weak field limit in $f(R)$-gravity.}

GR has been tested with increasing accuracy over the last 100 years. Its field equations are non linear, and analytic solutions are only found for systems with high degree of symmetry. For example, time independence and spatial isotropy of an isolated system leads to the Schwarzschild solution. Unfortunately, real systems are not very symmetric. For weak gravitational fields, the Newtonian effects could be orders of magnitude larger than the relativistic correction. For these systems, we can use approximated methods. The most common and useful are the weak field limit and the Post-Newtonian approximation. In the first approximation the field is described without making assumptions on the motion of particles. In the second, the expansion is carried out in the order of the velocity of particles to the speed of light. In such limits, any alternative relativistic theory of gravity must reproduce GR in order to recover the tight constraints at Solar system scale (Will, 2006). At galactic scales, ETGs modify the Newtonian gravitational potential. Effect that could explain the flatness of the rotation curves of spiral galaxies without any DM halo (Damour and Esposito-Farèse (1992), Will (2006), Capozziello et al. (2007)). As part of this thesis, we shall study if they are viable alternatives at the Solar System and cluster of galaxies scales. In the weak field limit, on scales of AUs, the corrections must be negligible but on cluster scales they could provide a viable alternative to cluster profiles dominated by DM (Capozziello et al. (2009a), De Martino et al. (2014)).

\subsubsection{Weak field limits}

In a self-gravitating, spherically symmetric and virialized system of particles of mass $M$, the velocities of these particles can be written as

$$
v^{2} \sim \frac{G M}{r}
$$

In the Newtonian limit, velocities are small compared to the speed of light $c$, and the potential is

$$
U \sim v^{2} \sim \mathcal{O}(2)
$$

Therefore, to construct a Newtonian approximation we must develop $g_{\mu \nu}$ up to $\mathcal{O}(2)$

$$
\begin{aligned}
& g_{00}(t, \mathbf{x})=1+g_{00}^{(2)}(t, \mathbf{x})+\mathcal{O}(4) \\
& g_{i j}(t, \mathbf{x})=-\delta_{i j}+g_{i j}^{(2)}(t, \mathbf{x})+\mathcal{O}(4), \\
& g_{i 0}(t, \mathbf{x})=\mathcal{O}(3)
\end{aligned}
$$


where the signature of the metric is $(-+++)$. For simplicity we set $c=1$. The metric can be re-written in terms of the potentials as (Weinberg, 1972)

$$
\begin{aligned}
& g_{00}(t, \mathbf{x})=-1+2 \Phi(t, \mathbf{x})+\mathcal{O}(4) \\
& g_{i j}(t, \mathbf{x})=-\delta_{i j}+2 \Psi(t, \mathbf{x})+\mathcal{O}(4) \\
& g_{i 0}(t, \mathbf{x})=\mathcal{O}(3)
\end{aligned}
$$

In this limit, the Ricci scalar can be written as

$$
R \sim R^{(2)}(t, \mathbf{x})+\mathcal{O}(4)
$$

At $\mathcal{O}(2)$-order in metric perturbations, in the Newtonian limit, eqs. (1.60) and (1.61) can be re-written by using the Lagrangian in eq. (1.62) as

$$
\begin{aligned}
& R_{00}^{(2)}-\frac{R^{(2)}}{2}-f_{0}^{\prime \prime} \nabla^{2} R^{(2)}=\mathcal{X} T_{00}^{(0)} \\
& -3 f_{0}^{\prime \prime} \nabla^{2} R^{(2)}-R^{(2)}=\mathcal{X} T^{(0)}
\end{aligned}
$$

where $\nabla$ is the Laplacian and $R_{00}^{(2)}=\nabla^{2} \Phi(t, \mathbf{x})$ (Weinberg, 1972). These expressions can be simplified since (a) we can set $f_{0}^{\prime}=1$ with an adequate choice of units and (b) as a result of (1.60), in the limit that the space-time is asymptotically flat, using eqs. (1.69), (1.70), and (1.71) we derive $f(0)=0$.

For a perfect fluid, the energy-momentum tensor is given by eq. (1.19). Assuming that the fluid is composed by dust $(p=0)$, the field equations read

$$
\begin{aligned}
& \nabla^{2} \Phi-\frac{R^{(2)}}{2}-f_{0}^{\prime \prime} \nabla^{2} R^{(2)}=\mathcal{X} \rho \\
& -3 f_{0}^{\prime \prime} \nabla^{2} R^{(2)}-R^{(2)}=\mathcal{X} \rho
\end{aligned}
$$

where $\rho$ is the mass density. It is important to stress that imposing $f_{0}^{\prime \prime}=0$, the Poisson equation $\nabla^{2} \Phi=4 \pi G \rho$ is recovered

$$
\begin{aligned}
& \nabla^{2} \Phi-\frac{R^{(2)}}{2}=\mathcal{X} \rho \\
& -R^{(2)}=\mathcal{X} \rho,
\end{aligned}
$$

that implies

$$
\nabla^{2} \Phi=\frac{\mathcal{X}}{2} \rho
$$

and we recover the Poisson equation. 


\subsubsection{The Post-Newtonian limits}

In the Post-Newtonian limit, one is interested in describing the motion of the particles and more in general of a system, beyond the Newtonian order by including corrections at the second and fourth order in the perturbation expansion of the metric. As shown by Stelle (1978), an $R^{2}$-theory of gravity introduces a Yukawa-like correction term in the Newtonian potential. Therefore, this correction also appears in any $f(R)$ model that can be expanded in Taylor series. The Yukawa correction introduces a characteristic scale length in the problem and the Gauss theorem is not locally valid. It is verified only asymptotically, when the Yukawa correction goes to zero ${ }^{8}$. However, the conservation laws preserved since the Bianchi identities are still valid.

In Appendix B we present the solution of the field equations in the weak field limit for a spherically symmetric matter distribution. Therefore, the Newtonian potential can be recast as

$$
\Phi(r)=-\left(\frac{G M(\leq r)}{f_{0}^{\prime} r}+\frac{L \delta_{1}(t) e^{-\frac{r}{L}}}{6 r}\right)
$$

where $\delta_{1}(t)$ is an arbitrary function of time that depends on the coefficients of the Taylor expansion of $f(R)$, and

$$
L=\sqrt{\frac{-f_{0}^{\prime}}{\left(6 f_{0}^{\prime \prime}\right)}} .
$$

The eq. (1.80) suggests redefining the gravitational constant as $G_{e f f}=G / f_{0}^{\prime}$. Only in GR, $f(R)=R$, one can recover the Newtonian potential in the Newtonian limit. The parameters $f_{0}^{\prime}, f_{0}^{\prime \prime}$, and the function $\delta_{1}(t)$ measure by how much the specifying $f(R)$-model deviates from GR. The parameter $L$ can be interpreted as an effective length, characteristic of the system. A more commonly used expression of eq. (1.80) is

$$
\Phi(r)=-\frac{G M(r)}{(1+\delta) r}\left(1+\delta e^{-\frac{r}{L}}\right)
$$

where the first term represents a Newtonian potential associated to baryonic point-like mass $M /(1+\delta)$. The parameters $\delta$ is related to the coefficients of the Taylor series (1.62) as follow

$$
f_{0}^{\prime}=1+\delta
$$

Moreover, eqs. (1.80) and (1.82) are equivalent when $\delta_{1}(t)$ is constant in time and $\delta_{1}=-\frac{6 G M(r)}{L^{2}} \frac{\delta}{1+\delta}$ and $L \propto \sqrt{-\delta /(1+\delta)}$.

The physical meaning of $L$ requires a detailed discussion. As pointed out by Capozziello and De Laurentis (2011), compared to GR, a theory of second order, $f(R)$

\footnotetext{
${ }^{8}$ The equivalence between a spherically symmetric distribution and point-like distribution is not valid, and how the matter is distributed in the space is very important.
} 
gravity is fourth-order and contains extra degrees of freedom that can be expressed as a new characteristic scale length in the weak field limit. In general, one can extend the paradigm for $(2 k+2)$-order theories of gravity saying that, increasing the order of theory of two derivation orders adds a characteristic scale length in Newtonian limit (Quandt and Schmidt, 1991). From this point of view, $L$ can be interpreted as an extra gravitational radius similar to the Schwarzschild radius. As a result, the gravitational interaction will depend on the size of self-gravitating system; gravity is not longer scale invariant, and the Gauss theorem holds only asymptotically. However, Bianchi identities hold for $f(R)$ as for any ETGs and conservation laws are verified like in GR.

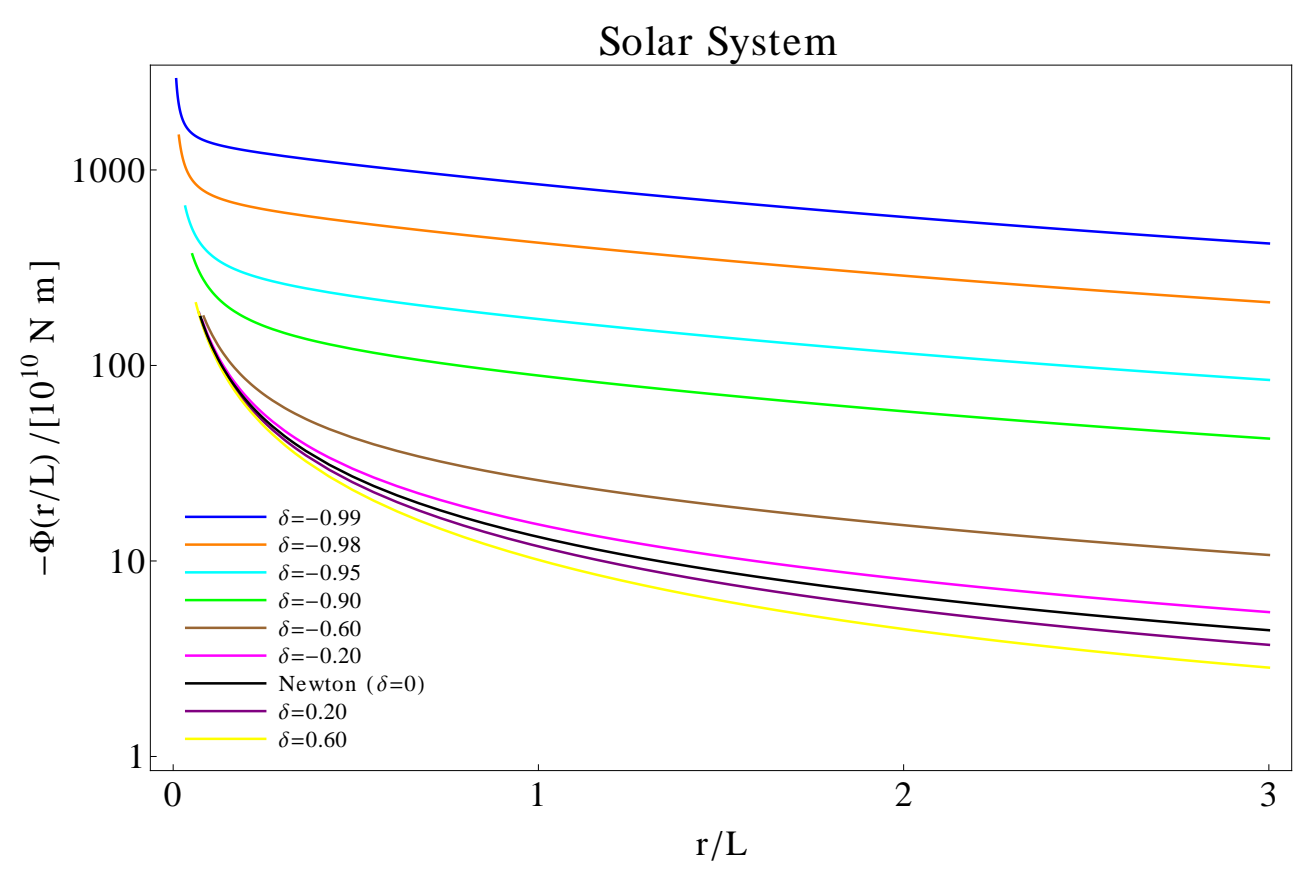

Figure 1.6: Modified Newtonian potential at the scale of the Solar System.

Based on physical arguments we can constrain the value of $\delta$ to be in the range $-1<\delta<1$. In fact if $\delta<-1$ the gravitational potential becomes repulsive. In the limit $\delta$ going to zero we recover the Newtonian potential, so even observations of the gravitational dynamics in the weak field limit can constraint ETGs. Indeed, any ETGs that differs too much from GR in the weak field limit is rejected by observational constraint. In Fig. 1.6, we show the modified Newtonian potential of eq. (1.82) at the scale of the Solar System $(L=1 \mathrm{AU}) . \delta$ varies in the range $[-1,1]$, and only for values very close to $\delta=0$ the modification at those scales start to be negligible.

Eq. (1.82) was used by Sanders (1984) to reproduce the rotation curves of spiral galaxies. For negative values $-1<\delta<0$ the Yukawa correction adds a repulsive term to the Newtonian gravitational potential an produces flat rotation curves in the limit $r \gg L$. Fitting the data of a small sample of spiral galaxies Sanders (1984) found $-0.95 \lesssim \delta \lesssim-0.92$. More recently, data on spirals (Capozziello et al. (2007), Cardone 
and Capozziello (2011)) and on velocity dispersion of elliptical galaxies (Napolitano et al., 2012) showed similar results. As part of this thesis, we will show that cluster pressure profiles (De Martino et al., 2014) also show similar constraints.

\subsection{Discussion and remarks}

The concordance $\Lambda \mathrm{CDM}$ model is supported by a wide range of observations. It describes the period of acceleration of our spatially flat Universe (Hinshaw et al. (2013), Planck Collaboration (2013j)). It requires two unknown matter components: DM that accounts for the clustering of galaxies and LSS, and a cosmological constant $\Lambda$ that dominates the energy budget and drives the present cosmic acceleration. From the theoretical point of view, a cosmological constant is problematic since it requires a high degree of fine-tuning in the initial conditions at the Planck scales. If the alternative to DM and a cosmological constant is to modifying GR the new theory could make predictions fundamentally different not just at cosmological scales, but at all scales. If the need to introduce DM and DE can be seen as a failure of GR to explain astrophysical and cosmological phenomena, not such failure exists at the well measured Solar System scales. Generically, ETGs predict Yukawa-like corrections of the Newtonian potential (Capozziello et al., 2009b) that will alter the dynamics on a regime where GR is very well tested. For this reason, a chameleon mechanism is introduced to mask its effects. Nevertheless, it could have an effect on the apparent anomalous longrange acceleration in the Pioneer 10/11, Galileo, and Ulysses spacecrafts, although a more conventional explanation has been widely accepted, alternatives exists using a modified theory of gravity (Anderson et al., 2002). Present and future dataset from observations of LSS, SNeIa, CMB, lensing, GWs, and experiments in particle physics, will be capable of confirming or ruling out both GR or ETGs. In this context, the goal of this thesis is to study various astrophysical phenomena from scale of a few AU to cosmological distances. Even at scales were gravity is well tested and GR describes the observations with great accuracy, it is important to study if ETGs describe, in some limit, the same phenomena with equal or better accuracy, without bringing strong modifications that would rule out these new descriptions of gravity. For this reason, we will start by analyzing phenomena on small scales, such as star formation and GWs emission from binary systems, to conclude with cosmological tests. 


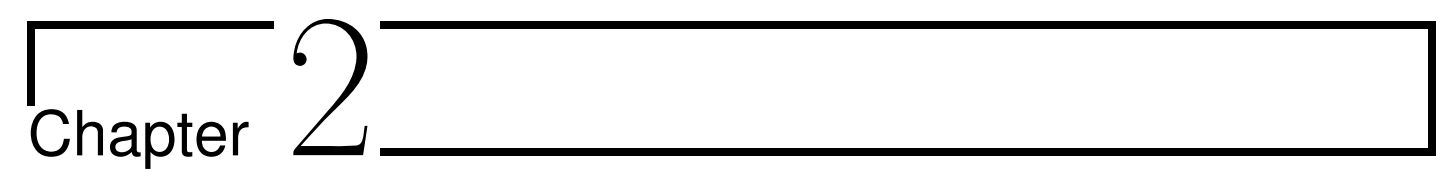

\title{
Jeans Instability in $f(R)$ theories of
}

\author{
gravity
}

\section{Contents}

2.1 Gravitational instability and star formation . . . . . . 32

2.2 The Modified Poisson equation in $f(R)$-gravity . . . . . 35

2.3 Jeans criteria for gravitational instability in $f(R)$-gravity $\quad \mathbf{3 6}$

2.4 The Jeans mass limit in $f(R)$-gravity . . . . . . . 38

2.4.1 The Jeans Mass - Temperature relation . . . . . . . . . . . 39

2.5 Discussion and future perspectives ........... 41

Ctellar astrophysics has been studied in the framework of $f(R)$-gravity in order to $\checkmark$ validate alternative theories of gravity at the Solar Systems scale, and to provide alternative explanations to peculiar objects (e.g. magnetars, stars in the instability strip, protostars, etc. (Cooney et al. (2010), Upadhye et al. (2010))) or to astrophysical processes such as star formation (Capozziello et al. (2011), Chang and Hui (2011), Capozziello et al. (2012)).

These models are not without difficulty: Kobayashi and Maeda (2008) claimed that in $f(R)$ gravity the dynamics of scalar fields prevents the formation of relativistic stars; nevertheless, analytic $f(R)$-models are naturally screened at those scales (see for more details Sect. 1.7.2) and in fact allow the formation of collapsed objects without requiring any ad-hoc screening mechanism.

In this chapter, we will study star formation in these alternative theories of gravity. We will analyze Jeans instability of self-gravitating systems in GR and in $f(R)$-gravity in order to see if ETGs lead to different results that could potentially serve as a test of these theories. We will study both the fluid and collisionless self-gravitating limits, and we will compute a new dispersion relation and, consequently, a new Jeans length for the instability in ETGs. 


\subsection{Gravitational instability and star formation}

Gravitational systems are usually treated as fluids. But galaxies are a mixture of several systems. Together with stars, the interstellar medium contains clouds of cold and ionized gas and dust, like Giant Molecular Clouds, HII regions, Bok Globules, etc. These clouds are usually stable, with pressure (due to a finite temperature) balancing self-gravity. But stellar clusters are better explained as collisionless systems. Although the physical processes of a collapsing clouds are well described in the fluid limit, it can not take into account some effects due to the particle nature of the interstellar medium (such as Landau Damping) that could much be larger when a different theory of gravitation is assumed. Therefore, we will carry out our study using the statistical mechanics approach.

The dynamic of self-gravitating collisionless systems is described by the the collisionless Boltzmann equation coupled with the Poisson equation.

$$
\begin{aligned}
& \frac{\partial f(\vec{r}, \vec{v}, t)}{\partial t}+\left(\vec{v} \cdot \vec{\nabla}_{r}\right) f(\vec{r}, \vec{v}, t)-\left(\vec{\nabla} \Phi(\vec{r}, \vec{v}, t) \cdot \vec{\nabla}_{v}\right) f(\vec{r}, \vec{v}, t)=0, \\
& \vec{\nabla}^{2} \Phi(\vec{r}, t)=4 \pi G \int f_{f}(\vec{r}, \vec{v}, t) d \vec{v} .
\end{aligned}
$$

If the system is in equilibrium then the distribution function $f^{(0)}(\vec{r}, v)$ is also time independent. Once the system undergoes a small perturbations these equations can be linearized and the small perturbations to the equilibrium can be written as

$$
\begin{aligned}
& f(\vec{r}, \vec{v}, t)=f^{(0)}(\vec{r}, \vec{v})+\epsilon f^{(1)}(\vec{r}, \vec{v}, t), \\
& \Phi(\vec{r}, t)=\Phi_{0}(\vec{r})+\epsilon \Phi_{1}(\vec{r}, t),
\end{aligned}
$$

where $\epsilon \ll 1$ is a small real number. $f^{(1)}$ and $\Phi_{1}$ are the perturbed distribution function and gravitational potential, respectively. Substitution in eqs. ((2.1), (2.2)) leads to (Binney and Tremaine, 1994)

$$
\begin{aligned}
& \frac{\partial f^{(1)}(\vec{r}, \vec{v}, t)}{\partial t}+\vec{v} \cdot \frac{\partial f^{(1)}(\vec{r}, \vec{v}, t)}{\partial \vec{r}}-\vec{\nabla} \Phi_{1}(\vec{r}, t) \cdot \frac{\partial f^{(0)}(\vec{r}, \vec{v})}{\partial \vec{v}}-\vec{\nabla} \Phi_{0}(\vec{r}) \cdot \frac{\partial f^{(1)}(\vec{r}, \vec{v}, t)}{\partial \vec{v}}=0 \\
& \vec{\nabla}^{2} \Phi_{1}(\vec{r}, t)=4 \pi G \int f^{(1)}(\vec{r}, \vec{v}, t) d \vec{v} .
\end{aligned}
$$

From these equations we can derive the dispersion relation that describes the growing and decaying solutions.

Before proceeding further, let us assume that a spherically symmetric and homogeneous cloud is in equilibrium at some initial density $\rho_{0}$ and pressure $p_{0}$. To simplify, 
the initial temperature is taken to be zero $v_{0}=0$. Jean's analysis has a well known drawback: since there are no internal motions in the initial configuration, the Euler equation requires the gravitational potential to be $\nabla \Phi_{0}=0$ while the Poisson equation requires $\nabla \Phi_{0}=4 \pi G \rho_{0}$, implying $\rho_{0}=0$. The overcome this inconsistency Jeans introduced the "Swindle" approximation. In this ad hoc assumption, the Poisson equation is assumed to describe the relation between perturbed quantities; the gravitational potential of the unperturbed matter distribution is arbitrarily set to zero. Clearly, there is no theoretical justification for this ansatz but in some cases is acceptable (Binney and Tremaine, 1994). Similarly, in our statistical approach the equilibrium state is assumed to be homogeneous and independent of time. By setting $f^{(0)}(\vec{x}, \vec{v}, t)=f^{(0)}(\vec{v})$, using the "Jeans Swindle" approximation and Fourier transforming the resulting equations we obtain

$$
\begin{aligned}
& -i \omega f^{(1)}+\vec{v} \cdot\left(i \vec{k} f^{(1)}\right)-\left(i \vec{k} \Phi_{1}\right) \cdot \frac{\partial f^{(0)}}{\partial \vec{v}}=0, \\
& -k^{2} \Phi_{1}=4 \pi G \int f^{(1)} d \vec{v},
\end{aligned}
$$

that yield the following dispersion relation

$$
1+\frac{4 \pi G}{k^{2}} \int \frac{\vec{k} \cdot \frac{\partial f^{(0)}}{\partial \vec{v}}}{\vec{v} \cdot \vec{k}-\omega} d \vec{v}=0 .
$$

At the conditions of the Interstellar Medium, temperature and number density are $T \sim 20 \mathrm{~K}$ and $n \sim 10^{8} \mathrm{~m}^{-3}$. If the cloud is in thermodynamical equilibrium we can assume that $f^{(0)}$ is given by the Maxwell-Boltzmann distribution

$$
f^{(0)}=\frac{\rho_{0}}{\left(2 \pi \sigma^{2}\right)^{\frac{3}{2}}} e^{-\frac{v^{2}}{2 \sigma^{2}}}
$$

where $\sigma$ is the dispersion velocity due to the mean temperature of the system. Choosing a reference frame were $\vec{k}=(k, 0,0)$ and substituting in eq. (2.9), we obtain

$$
1-\frac{2 \sqrt{2 \pi} G \rho_{0}}{k \sigma^{3}} \int \frac{v_{x} e^{-\frac{v_{x}^{2}}{2 \sigma^{2}}}}{k v_{x}-\omega} d v_{x}=0 .
$$

The stability limit is obtained by setting $\omega=0$

$$
k^{2}(\omega=0)=\frac{4 \pi G \rho_{0}}{\sigma^{2}}=k_{J}^{2}
$$

This formula defines the Jeans length of a collisionless system. In the fluid the result would be similar except that the velocity dispersion $\sigma$ is substituted by the sound speed $c_{s}$ of the medium. Eq. (2.11) indicates that in a clouds all perturbations with wavelengths $\lambda>\lambda_{J}$ (where $\lambda=2 \pi / k$ ) would be unstable. 
To evaluate the integral in eq. (2.11) for real, nonzero values of $\omega$, one must decide how to integrate around the singularity at $\omega=k v_{x}$. To study the instability, one can rewrite the dispersion relation as

$$
1-\frac{k_{J}^{2}}{k^{2}} W(Z)=0
$$

defining the quantity

$$
W(Z) \equiv \frac{1}{\sqrt{2 \pi}} \int \frac{x e^{-\frac{x^{2}}{2}}}{x-Z} d x,
$$

where $Z=\omega / k \sigma$. For the unstable modes, one can set $\omega=i \omega_{I}$ and $\operatorname{Re}(W(Z))=0$ (see Appendix $\mathrm{C}$ for more details), and substituting the following identities

$$
\begin{aligned}
& \int_{0}^{\infty} \frac{x^{2} e^{-x^{2}}}{x^{2}+\beta^{2}} d x=\frac{1}{2} \sqrt{\pi}-\frac{1}{2} \pi \beta e^{\beta^{2}}[1-\operatorname{erf}(\beta)], \\
& \operatorname{erf}(\beta)=\frac{2}{\sqrt{\pi}} \int_{0}^{z} e^{-t^{2}} d t
\end{aligned}
$$

in the dispersion relation, we obtain

$$
k^{2}=k_{J}^{2}\left\{1-\sqrt{\pi} \frac{\omega_{I}}{\sqrt{2} k \sigma} e^{\left(\frac{\omega_{I}}{\sqrt{2} k \sigma}\right)^{2}}\left[1-\operatorname{erf}\left(\frac{\omega_{I}}{\sqrt{2} k \sigma}\right)\right]\right\}
$$

In Fig. 2.1 we represent the dispersion relation in the fluid limit $\omega=k^{2}-k_{J}^{2}$ (red solid line) and in the collisionless limit (eq. (2.17)) (blue solid line). For $\lambda<\lambda_{J}$ pressure builds up sufficiently fast to stop the growth of density perturbations. In a fluid, the perturbations propagate as sound waves, while on a collisionless stellar system the operating mechanisms is Landau-damping. In summary, the stability of an infinite homogeneous stellar system is very close to that of a fluid. In both case there is an instability when $k<k_{J}$. From this analysis, it is straightforward to obtain the minimum mass for an overdensity to collapse. This Jeans Mass scale is obtained by setting $\omega=0$ (see (2.12)). It is defined as the mass contained within a sphere of diameter $\lambda_{J}$ :

$$
M_{J}=\frac{4 \pi}{3} \rho_{0}\left(\frac{1}{2} \lambda_{J}\right)^{3}
$$

where

$$
\lambda_{J}^{2}=\frac{\pi \sigma^{2}}{G \rho_{0}}
$$

is the Jeans length. By substituting eq. (2.19) in eq. (2.18) we recover

$$
M_{J}=\frac{\pi}{6} \sqrt{\frac{1}{\rho_{0}}\left(\frac{\pi \sigma^{2}}{G}\right)^{3}} .
$$

As an example, let us compute the Jeans Mass of a diffuse cloud of pure $\mathrm{H}$ with temperature $T=50 \mathrm{~K}$ and number density $n=5 \cdot 10^{8} \mathrm{~m}^{-3}$. Then $\rho_{0}=m_{H} n_{H}=$ 


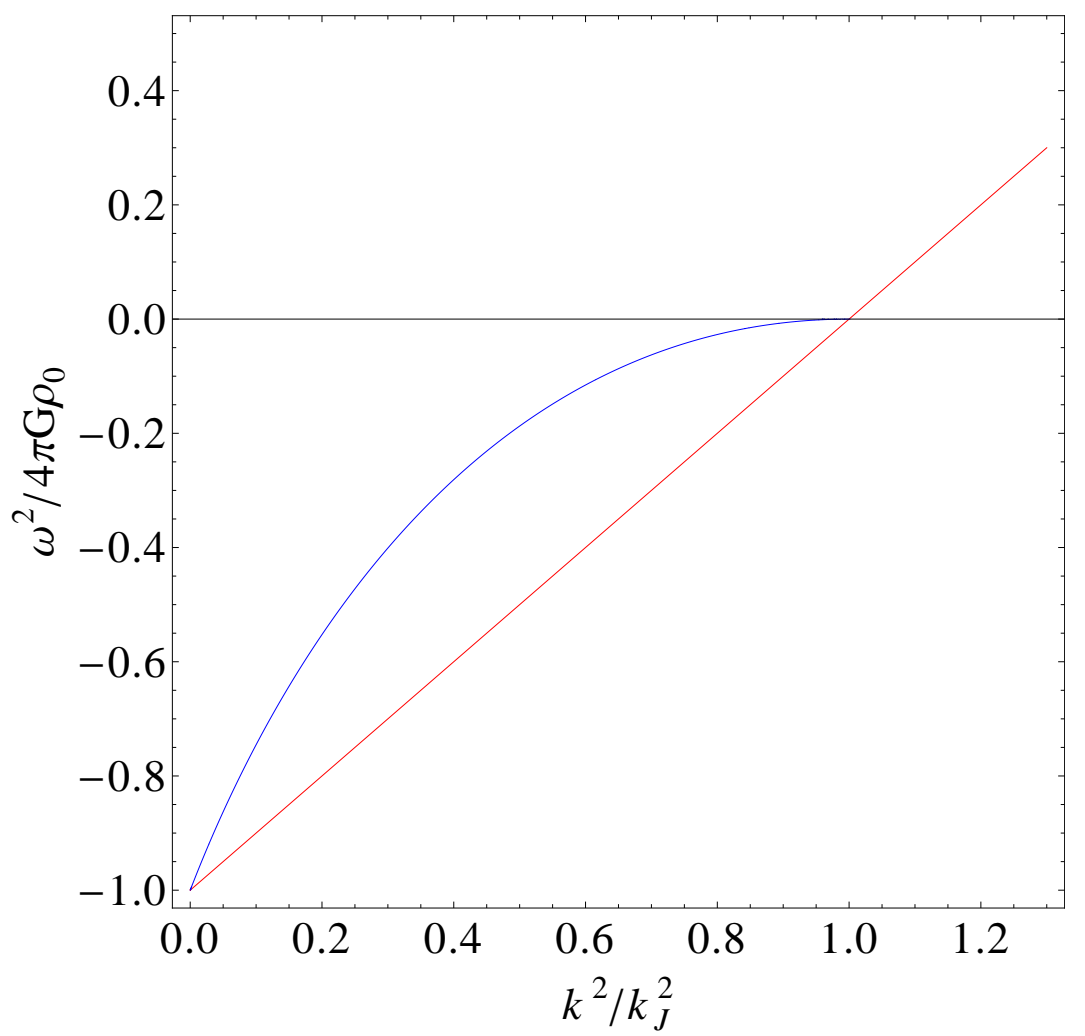

Figure 2.1: The dispersion relation for an infinite homogeneous fluid (red line) and an infinite collisionless stellar system (blue line).

$8.4 \cdot 10^{-19} \mathrm{~kg} \mathrm{~m}^{-3}$. From eq. $(2.20), M_{J} \sim 1500 M_{\odot}$. This is a factor $10-1000$ times smaller than the mass of typical HI clouds. These clouds are unstable to gravitational collapse and are the natural regions of star formation. Let us now study how ETGs modify the dispersion relation. We shall demonstrate that eq. (2.17) is the GR limit of a more general relation.

\subsection{The Modified Poisson equation in $f(R)$-gravity}

Starting from the field equations (1.75) and (1.76), one can write a modified Poisson equations for $f(R)$-gravity. Let us start by restoring in eqs. (1.75) and (1.76) the speed of light $c$

$$
\begin{aligned}
& \frac{1}{c^{2}} \nabla^{2} \Phi-\frac{R^{(2)}}{2}-f_{0}^{\prime \prime} \nabla^{2} R^{(2)}=\mathcal{X} \rho c^{2}, \\
& -3 f_{0}^{\prime \prime} \nabla^{2} R^{(2)}-R^{(2)}=\mathcal{X} \rho c^{2} .
\end{aligned}
$$

Inserting eq. (2.22) in eq. (2.21) we obtain

$$
\nabla^{2} \Phi-\frac{c^{2} R^{(2)}}{6}=\frac{16}{3} \pi G \rho
$$


Remembering that

$$
R^{(2)} \simeq \frac{1}{2} \nabla^{2} g_{00}^{(2)}-\frac{1}{2} \nabla^{2} g_{i i}^{(2)}
$$

and using the metric approximation of eqs. (1.69), (1.70), and (1.71) then

$$
R^{(2)} \simeq \nabla^{2}(\Phi-\Psi)
$$

where the potential $\Psi$ is related to the metric components $g_{i i}^{(2)}$. It represents a relativistic degree of freedom in the weak field limit (Weinberg, 1972). Introducing eq. (2.25) in eqs. (2.21) and (2.22), the field equations now read

$$
\begin{aligned}
& \nabla^{2} \Phi+\nabla^{2} \Psi-2 f_{0}^{\prime \prime} \nabla^{4} \Phi+2 f_{0}^{\prime \prime} \nabla^{4} \Psi=2 \mathcal{X} \rho \\
& \nabla^{2} \Phi-\nabla^{2} \Psi+3 f_{0}^{\prime \prime} \nabla^{4} \Phi-3 f_{0}^{\prime \prime} \nabla^{4} \Psi=-\mathcal{X} \rho .
\end{aligned}
$$

Let us remark again that by setting $f_{0}^{\prime \prime}=0$ we recover the standard Poisson (see Sect. 1.7.1). Equations (2.26) and (2.27) allow us to re-analyze the Jeans Instability in the statistical mechanic approach following similar arguments than those presented in Sect. 2.1

\subsection{Jeans criteria for gravitational instability in $f(R)$ - gravity}

Following Sect. 2.1 we introduce the collisionless Boltzmann and Poisson equations. In $f(R)$-gravity we have two coupled equations to describe the two gravitational potentials $((2.26)$ and $(2.27))$. These equations are

$$
\begin{aligned}
& \frac{\partial f(\vec{r}, \vec{v}, t)}{\partial t}+\left(\vec{v} \cdot \vec{\nabla}_{r}\right) f(\vec{r}, \vec{v}, t)-\left(\vec{\nabla} \Phi \cdot \vec{\nabla}_{v}\right) f(\vec{r}, \vec{v}, t)=0, \\
& \nabla^{2}(\Phi+\Psi)-2 f_{0}^{\prime \prime} \nabla^{4}(\Phi-\Psi)=16 \pi G \int f(\vec{r}, \vec{v}, t) d \vec{v}, \\
& \nabla^{2}(\Phi-\Psi)+3 f_{0}^{\prime \prime} \nabla^{4}(\Phi-\Psi)=-8 \pi G \int f(\vec{r}, \vec{v}, t) d \vec{v} .
\end{aligned}
$$

Following the procedure outlined in Sect. 2.1 we consider first order perturbations, represented by $f^{(1)}$, to the initial state of equilibrium. The linearized system becomes

$$
\begin{aligned}
& \frac{\partial f^{(1)}(\vec{r}, \vec{v}, t)}{\partial t}+\vec{v} \cdot \frac{\partial f^{(1)}(\vec{r}, \vec{v}, t)}{\partial \vec{r}}-\vec{\nabla} \Phi_{1}(\vec{r}, t) \cdot \frac{\partial f^{(0)}(\vec{r}, \vec{v})}{\partial \vec{v}}+ \\
& -\vec{\nabla} \Phi_{0}(\vec{r}) \cdot \frac{\partial f^{(1)}(\vec{r}, \vec{v}, t)}{\partial \vec{v}}=0,
\end{aligned}
$$




$$
\begin{aligned}
& \nabla^{2} \Phi_{1}+\nabla^{2} \Psi_{1}-2 f_{0}^{\prime \prime} \nabla^{4} \Phi_{1}+2 f_{0}^{\prime \prime} \nabla^{4} \Psi_{1}=16 \pi G \int f^{(1)}(\vec{r}, \vec{v}, t) d \vec{v}, \\
& \nabla^{2} \Phi_{1}-\nabla^{2} \Psi_{1}+3 f_{0}^{\prime \prime} \nabla^{4} \Phi_{1}-3 f_{0}^{\prime \prime} \nabla^{4} \Psi_{1}=-8 \pi G \int f^{(1)}(\vec{r}, \vec{v}, t) d \vec{v},
\end{aligned}
$$

Like in standard GR, we use the "Jeans Swindle" approximation and we set $\Phi_{0}(\vec{r})=0$ and $\Psi_{0}(\vec{r})=0$. In Fourier space we have

$$
\begin{aligned}
& -i \omega f^{(1)}+\vec{v} \cdot\left(i \vec{k} f^{(1)}\right)-\left(i \vec{k} \Phi_{1}\right) \cdot \frac{\partial f^{(0)}}{\partial \vec{v}}=0, \\
& -k^{2}\left(\Phi_{1}+\Psi_{1}\right)-2 f_{0}^{\prime \prime} k^{4}\left(\Phi_{1}-\Psi_{1}\right)=16 \pi G \int f^{(1)} d \vec{v}, \\
& k^{2}\left(\Phi_{1}-\Psi_{1}\right)-3 f_{0}^{\prime \prime} k^{4}\left(\Phi_{1}-\Psi_{1}\right)=8 \pi G \int f^{(1)} d \vec{v} .
\end{aligned}
$$

A relation between $\Phi_{1}$ and $\Psi_{1}$, in Fourier space, can be obtained by combining eqs. (2.35) and (2.36)

$$
\Psi_{1}=\frac{3-4 f_{0}^{\prime \prime} k^{2}}{1-4 f_{0}^{\prime \prime} k^{2}} \Phi_{1}
$$

then, inserting this relation in eq. (2.35) and combining it with Eq. (2.34), after some algebra, we can write the dispersion relation as

$$
1-4 \pi G \frac{1-4 f_{0}^{\prime \prime} k^{2}}{3 f_{0}^{\prime \prime} k^{4}-k^{2}} \int\left(\frac{\vec{k} \cdot \frac{\partial f^{(0)}}{\partial \vec{v}}}{\vec{v} \cdot \vec{k}-\omega}\right) d \vec{v}=0 .
$$

Following the same arguments than in the GR case, let us assume that the system is in thermal equilibrium and the distribution function is well approximated by a Maxwell distribution. Again, we choose a reference frame so $\vec{k}=(k, 0,0)$; then eq. (2.38) becomes

$$
1+\frac{2 \sqrt{2 \pi} G \rho_{0}}{\sigma^{3}} \frac{1-4 f_{0}^{\prime \prime} k^{2}}{3 f_{0}^{\prime \prime} k^{4}-k^{2}}\left[\int \frac{k v_{x} e^{-\frac{v_{x}^{2}}{2 \sigma^{2}}}}{k v_{x}-\omega} d v_{x}\right]=0 .
$$

If we set $f_{0}^{\prime \prime}=0$ we recover the GR result of eq. (2.11). The integration of (2.39) is performed as in eq. (2.11) (see Appendix C) and let us quote the result here

$$
1+\mathcal{G} \frac{1-4 f_{0}^{\prime \prime} k^{2}}{3 f_{0}^{\prime \prime} k^{4}-k^{2}}\left[1-\sqrt{\pi} x e^{x^{2}}(1-\operatorname{erf}[x])\right]=0
$$

where $x=\frac{\omega_{I}}{\sqrt{2} k \sigma}$ and $\mathcal{G}=\frac{4 G \pi \rho_{0}}{\sigma^{2}}$.

To make an easier comparison between the GR and the $f(R)$ solutions we normalize the dispersion relation of the classical Jeans length of eq. (2.19). This can be done by fixing the parameter of $f(R)$-gravity as

$$
f_{0}^{\prime \prime}=-\frac{1}{k_{J}^{2}}=-\frac{\sigma^{2}}{4 \pi G \rho_{0}}
$$


In this particular case the dispersion relation reads

$$
\frac{3 k^{4}}{k_{j}^{4}}+\frac{k^{2}}{k_{j}^{2}}=\left(\frac{4 k^{2}}{k_{j}^{2}}+1\right)\left[1-\sqrt{\pi} x e^{x^{2}}(1-\operatorname{erf}[x])\right]=0 .
$$

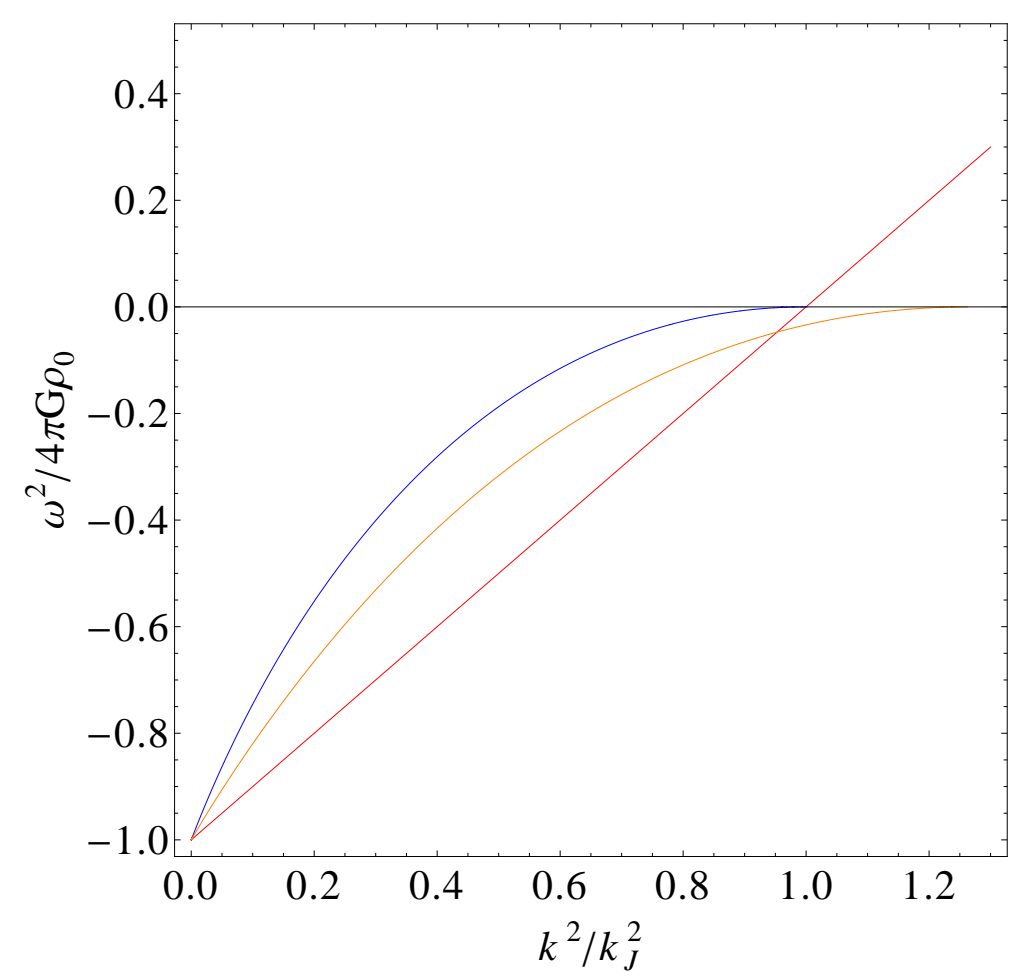

Figure 2.2: Red and blue lines represent the dispersion relations of the fluid and statistical mechanic limit in GR (see Fig. 2.1. The orange line represents the dispersion relation of (2.42) for the value for $f_{0}^{\prime \prime}$ given by $(2.41)$.

In Fig. 2.2 we compare the new solution of eq. (2.42) and our earlier result of (2.17). The red line gives the the differences between $f(R)$ and Newtonian gravity. The red, blue and orange lines represent the dispersion relation in the fluid limit and the statistical limit of GR and $f(R)$, respectively. Figure 2.2 shows than a different theory of gravity changes the limit of instability. The limit in $f(R)$ is higher than the classical case (blue and red lines). Therefore, the Jeans mass decreases in ETGs and changes the initial conditions at the start of the collapse in interstellar clouds. We will expand this argument in the following section.

\subsection{The Jeans mass limit in $f(R)$-gravity}

Following the analysis in the Newtonian gravity we compute the Jeans mass within a sphere of diameter the Jeans length. The Jeans length is obtained by solving eq. (2.42) with the condition $\omega=0$. The collapse in ETGs occurs for

$$
\tilde{k}_{J}^{2}=1.2637 k_{J}^{2}
$$


Alternatively, we can obtain an analytical expression by solving eq. (2.39) with $\omega=0$. The solution is

$$
3 \sigma^{2} f_{0}^{\prime \prime} k^{4}-\left(16 \pi G \rho_{0} f_{0}^{\prime \prime}+\sigma^{2}\right) k^{2}+4 \pi G \rho_{0}=0 .
$$

As indicated in Sec 1.6.2 we consider only models with $f_{0}^{\prime \prime}<0$, so eq. (2.44) present solutions for real values of $k$. The numerical solution of eq. (2.43) can be expressed as

$$
\tilde{k}_{J}^{2}=\frac{2}{3}(3+\sqrt{21}) \pi \frac{G \rho}{\sigma^{2}} .
$$

The relation with the Newtonian value of the Jeans instability is

$$
\tilde{k}_{J}^{2}=\frac{1}{6}(3+\sqrt{21}) k_{J}^{2}
$$

From this scale we can show that the Jeans mass is

$$
\tilde{M}_{J}=6 \sqrt{\frac{6}{(3+\sqrt{21})^{3}}} M_{J},
$$

proportional to the standard Newtonian value. This last expression can be used to predict the Jeans Mass in different astrophysical environments.

\subsubsection{The Jeans Mass - Temperature relation}

In modern astrophysics the term "star formation" indicates the processes from which dense regions within molecular clouds collapse to form stars. Multi-wavelength observations and numerical modeling are key to study star formation (Falle (2007), Klessen et al. (2009)). Due to observational limitations the process is rather uncertain. Models consider the formation of individual stars and the formation of stellar clusters (McKee and Ostriker, 2007). The actual physical process are strongly influenced by the environment. The physical and chemical properties of the Interstellar Medium (ISM) in the Galaxy could be very different going from hot X-ray emitting plasma to cold molecular gas. As a first approximation it is possible to distinguish between (see Scheffler and Elsasser (1982), Kippenhahn and Weigert (1990), Dopita and Sutherland (2003), Carroll and Ostlie (2007) for details):

- Diffuse Hydrogen Clouds. Cold clouds of gas with temperature in the range $10 \div 50 \mathrm{~K}$ and up to $50 \div 100 \mathrm{kpc}$ away from galactic center. They can be studied by their $21 \mathrm{~cm}$ line emission of $\mathrm{HI}$.

- Diffuse Molecular Clouds are generally self-gravitating, magnetized, turbulent fluids systems, observed in the sub-mm range. Most of the molecular gas is $\mathrm{H}_{2}$ and the rest is CO. The physical conditions are very similar to those of HI clouds except these clouds are more massive. Typically, the mass is in the range $3 \div 100 M_{\odot}$, the temperature in $15 \div 50 \mathrm{~K}$ and the particle density in $(5 \div 50) \times 10^{8}$ $\mathrm{m}^{-3}$. 
- Giant Molecular Clouds are large complexes of dust and gas with masses $10^{5} \div 10^{6} M_{\odot}$. The temperature is $\sim 15 \mathrm{~K}$, and the number density of particles is $(1 \div 3) \times 10^{8} \mathrm{~m}^{-3}$ (Blitz et al. (1984), Lada and Kylafis (1999), McKee and Ostriker (2007)).

- HII regions. Gas clouds with temperatures in the range $10^{3} \div 10^{4} \mathrm{~K}$, emitting primarily in radio and IR. At low frequencies, emission is associated to free-free electron transitions (thermal Bremsstrahlung). Their densities range from over a $10^{6}$ particles per $\mathrm{cm}^{3}$ in the ultra-compact HII regions to only a few particles per $\mathrm{cm}^{3}$ in the largest and most extended areas. Their total masses range between $10^{2}$ and $10^{5} \mathrm{M}_{\odot}$ (Anderson et al., 2009).

- Bok Globules are dark clouds of dense cosmic dust and gas in which star formation could take place. They are found within HII regions and typically have masses between 2 and $50 \mathrm{M}_{\odot}$ contained within a region of about one light year.

We can compute the Jeans Mass for the different environments using the results of the previous section. Let us define the ISM density $\rho_{0}$ and velocity dispersion $\sigma$ as

$$
\rho_{0}=m_{H} n_{H} \mu, \quad \sigma^{2}=\frac{k_{B} T}{m_{H}}
$$

where $n_{H}$ is the number density of particles, $\mu$ the mean molecular weight, $k_{B}$ the Boltzmann constant and $m_{H}$ is the proton mass. The Jeans mass of eq. (2.20) now reads

$$
M_{J}=\frac{\pi}{6} \sqrt{\frac{1}{\rho_{0}}\left(\frac{\pi k_{B} T}{G m_{H}}\right)^{3}},
$$

In Tab.2.1 and Fig.2.3 we compare the Jeans Mass in GR and $f(R)$ for the different interstellar clouds. Let us remark that by altering the dynamics of the gravitational field, smaller fragments will collapse in $f(R)$ than in the standard Newtonian gravity.

\begin{tabular}{|l|ccccc|}
\hline \hline Subject & $\begin{array}{c}\mathrm{T} \\
(\mathrm{K})\end{array}$ & $\begin{array}{c}\mathrm{n} \\
\left(10^{8} \mathrm{~m}^{-3}\right)\end{array}$ & $\mu$ & $\begin{array}{c}M_{J} \\
\left(M_{\odot}\right)\end{array}$ & $\begin{array}{c}\tilde{M}_{J} \\
\left(M_{\odot}\right)\end{array}$ \\
\hline \hline Diffuse Hydrogen Clouds & 50 & 5.0 & 1 & 795.13 & 559.68 \\
Diffuse Molecular Clouds & 30 & 50 & 2 & 82.63 & 58.16 \\
Giant Molecular Clouds & 15 & 1.0 & 2 & 206.58 & 145.41 \\
Bok Globules & 10 & 100 & 2 & 11.24 & 7.91 \\
\hline \hline
\end{tabular}

Table 2.1: Jeans masses derived from Eq. (2.20) (Newtonian gravity) and $(2.47)(f(R)$ gravity). 


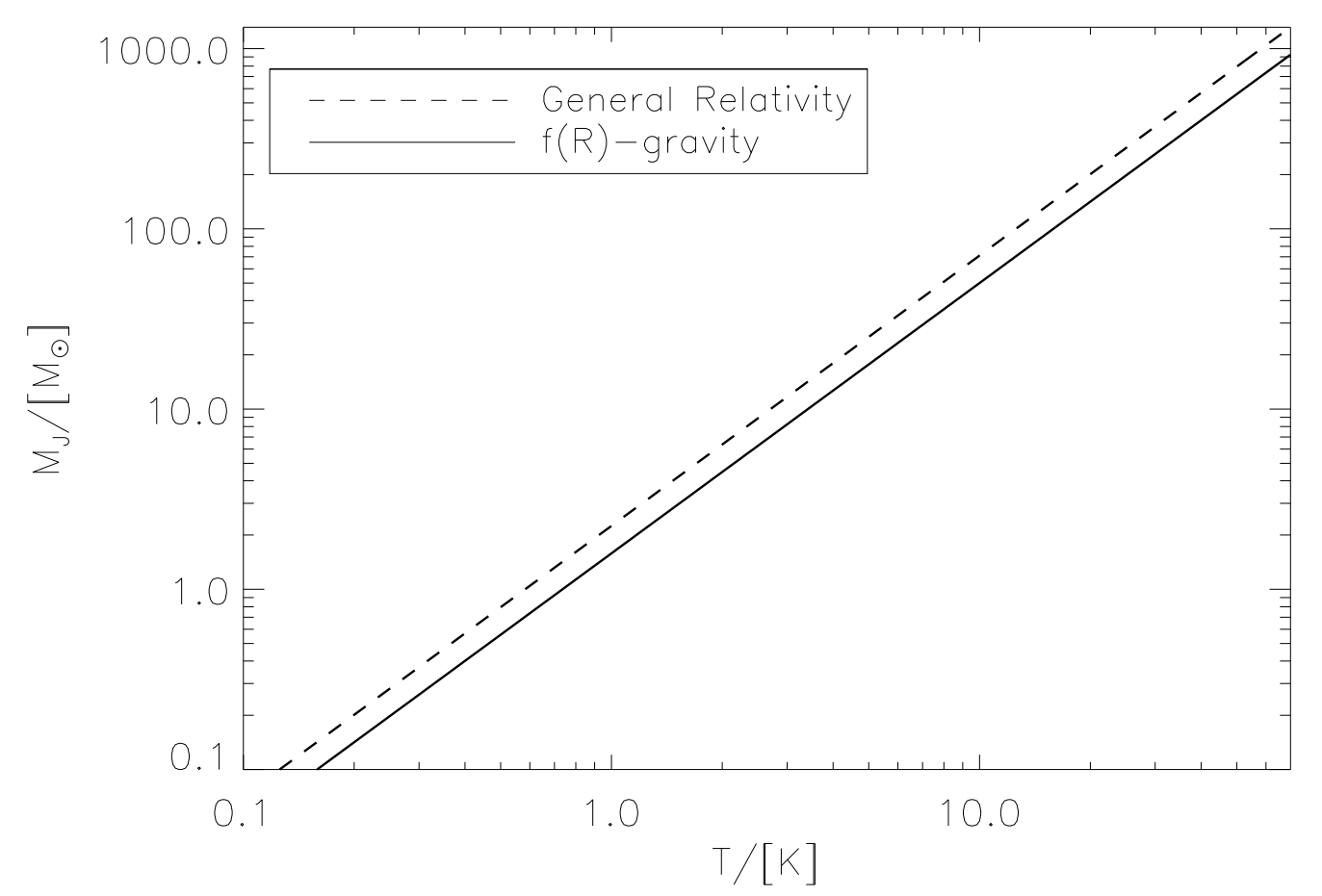

Figure 2.3: $M_{J}-T$ relation for the interstellar medium. The solid line corresponds to $f(R)$ gravity and the dashed-line to Newtonian gravity.

In Table 2.2 we present the physical properties of a subsample of molecular clouds from the catalog of Roman-Duval et al. (2010) and their corresponding Jeans masses computed in Newtonian gravity and $f(R)$ (eqs. (2.20) and (2.47)). In all case we the different is substantial. Modifying the description of the gravitational field changes the Jeans Mass. Since it is smaller in modified theories of gravity than in Newtonian gravity, star formation will be more efficient in the former than in the later.

\subsection{Discussion and future perspectives}

In this Chapter we have analyzed how by modifying our description of gravity, the conditions of star formation change. This is our first step of our goal of describing how different astronomical phenomena depend on the underlying gravitational field. Here, we have studied how Jeans instability was modified. We have derived the Poisson and Boltzmann equations for collisionless systems in the Newtonian limit of $f(R)$-gravity (eqs. (2.26) and (2.27)). We have obtained a new dispersion relation (2.40) that defines the instability criteria. We have shown that the classic result is recovered in the appropriate limit, by setting $f_{0}^{\prime \prime}=0$. For a particular case we obtained analytic expressions for the Jeans length and Jeans mass. The comparison of the statistical mechanics approach in standard gravity and in $f(R)$ is given in Fig. 2.2. In general, the 


\begin{tabular}{|l|cccc|}
\hline \hline Objects & $\mathrm{T}$ & $\mathrm{n}$ & $M_{J}$ & $\tilde{M}_{J}$ \\
& $(\mathrm{~K})$ & $\left(10^{8} \mathrm{~m}^{-3}\right)$ & $\left(M_{\odot}\right)$ & $\left(M_{\odot}\right)$ \\
\hline \hline GRSMC G053.59+00.04 & 5.97 & 1.48 & 18.25 & 12.85 \\
GRSMC G049.49-00.41 & 6.48 & 1.54 & 21.32 & 15.00 \\
GRSMC G018.89-00.51 & 6.61 & 1.58 & 22.65 & 15.94 \\
GRSMC G030.49-00.36 & 7.05 & 1.66 & 22.81 & 16.06 \\
GRSMC G035.14-00.76 & 7.11 & 1.89 & 28.88 & 20.33 \\
GRSMC G034.24+00.14 & 7.15 & 2.04 & 29.61 & 20.84 \\
GRSMC G019.94-00.81 & 7.17 & 2.43 & 29.80 & 20.98 \\
GRSMC G038.94-00.46 & 7.35 & 2.61 & 31.27 & 22.01 \\
GRSMC G053.14+00.04 & 7.78 & 2.67 & 32.06 & 22.56 \\
GRSMC G022.44+00.34 & 7.83 & 2.79 & 32.78 & 23.08 \\
GRSMC G049.39-00.26 & 7.90 & 2.81 & 35.64 & 25.09 \\
GRSMC G019.39-00.01 & 7.99 & 2.87 & 35.84 & 25.23 \\
GRSMC G034.74-00.66 & 8.27 & 3.04 & 36.94 & 26.00 \\
GRSMC G023.04-00.41 & 8.28 & 3.06 & 38.22 & 26.90 \\
GRSMC G018.69-00.06 & 8.30 & 3.62 & 40.34 & 28.40 \\
GRSMC G023.24-00.36 & 8.57 & 3.75 & 41.10 & 28.93 \\
GRSMC G019.89-00.56 & 8.64 & 3.87 & 41.82 & 29.44 \\
GRSMC G022.04+00.19 & 8.69 & 4.41 & 47.02 & 33.10 \\
GRSMC G018.89-00.66 & 8.79 & 4.46 & 47.73 & 33.60 \\
GRSMC G023.34-00.21 & 8.87 & 4.99 & 48.98 & 34.48 \\
GRSMC G034.99+00.34 & 8.90 & 5.74 & 50.44 & 35.50 \\
GRSMC G029.64-00.61 & 8.90 & 6.14 & 55.41 & 39.00 \\
GRSMC G018.94-00.26 & 9.16 & 6.16 & 55.64 & 39.16 \\
GRSMC G024.94-00.16 & 9.17 & 6.93 & 56.81 & 39.99 \\
GRSMC G025.19-00.26 & 9.72 & 7.11 & 58.21 & 40.97 \\
\hline \hline
\end{tabular}

Table 2.2: Physical properties and Jeans masses of a subsample of molecular clouds from the catalog of Roman-Duval et al. (2010)

Jeans mass is lower in $f(R)$ than in GR, speeding up the fragmentation of molecular class and favoring the formation of stars.

The approach developed by us can be generalized for other theories of modified gravity like non-local gravity, Gauss-Bonnet, string-inspired gravity and other approaches. In these cases, the resulting Poisson equation could be even more complicated due to the presence of extra scalar fields. The bottom line is that we have design a test that could discriminate between models of gravity. To facilitate a comparison with observations we need to include other physical effects that also affect the formation of stars, such as magnetic fields, turbulence and collisions. It is important to study stellar phenomena using $f(R)$-gravity in order to test the predictions with observations. 
For this reason, in the next chapter we will the gravitational waves emission from a binary system. In this case we will be exploring gravitational phenomena in strong field regime. 


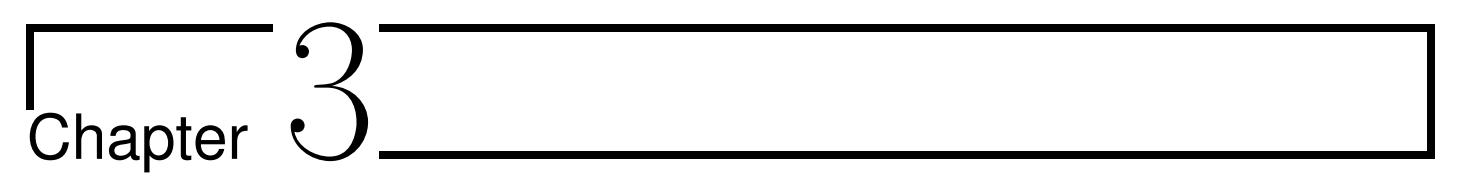

\section{Gravitational Wave emission in Extended Theories of Gravity}

\section{Contents}

3.1 Gravitational Waves: general framework and emission from binary systems . . . . . . . . . . . . 47

3.1.1 Application to binary systems . . . . . . . . . . . . 50

3.2 Energy-momentum tensor and quadrupolar emission in

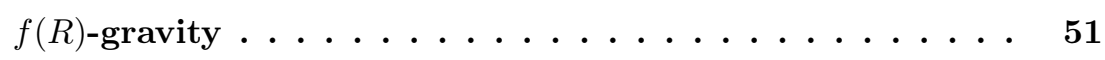

3.3 First time derivative of the orbital period in $f(R)$-gravity $\quad \mathbf{5 2}$

3.4 Constraints on ETGs from binary systems . . . . . . 53

3.5 Massive or massless gravitons? . . . . . . . . 57

3.5.1 An upper limit on the graviton mass from PSR J0348 + 0432. 57

3.6 Conclusions and future perspectives .......... 58

Tinstein's theory of gravity is a very good description of local gravitational phe1 nomena. After one century, theoretical predictions and measurements remain in very good agreement, in particular at the scale of the Solar System (Will, 2006). In Ch.1, we pointed out that at astrophysical and cosmological scales the need for DM and DE could be understood as shortcomings of the theory. Alternative theories have to provide equal or better description of the observations at all scales. Extended Theory of Gravities have been compared with data at many different scales (Allemandi et al. (2005), Capozziello and Troisi (2005), Capozziello et al. (2009a), Berry and Gair (2011), Capozziello et al. (2011), Cardone and Capozziello (2011), Capozziello et al. (2012), Amendola and Euclid Theory Working Group (2013), De Martino et al. (2014)). In this Chapter we will devote our analysis to Gravitational Waves (GWs) emission.

In the GR framework, the linearized field equations show that small perturbations of the metric propagate as waves (Weinberg (1972), Maggiore (2007)). The gravi- 
tational interaction is mediated by a single massless boson known as graviton. By contrast, in ETGs the extra degrees of freedom allow the existence of massive gravitational modes. A massive graviton mode affects the waveforms and polarization states of GWs and so, it can be used to test ETGs (Capozziello et al., 2008, Capozziello and De Laurentis, 2011). These modes have already been studied in the literature; for instance, the orbital motion of planets in the Solar System led to a constraint on the graviton mass of $m_{g} \leq 10^{-21} \mathrm{eV}$ (Will, 1998); from clusters of galaxies, Goldhaber and Nieto (1974) set the upper limit on $2 \times 10^{-29} \mathrm{heV}$, where $h$ is the Hubble constant in units of $100 \mathrm{~km} \mathrm{~s}^{-1} \mathrm{Mpc}^{-1}$ while from the power spectrum of weak lensing the upper limit is $m_{g}<7 \times 10^{-32} \mathrm{eV}$ (Choudhury et al., 2004). The first estimation of graviton mass from the emission of GWs in binary systems led to the upper limit $7.6 \times 10^{-20} \mathrm{eV}$ (Will, 2006), that is weaker but consistent with the others.

Currently, GWs emission has been indirectly detected in binary systems of collapsed objects by means of timing data in binary pulsars like the Hulse-Taylor pulsar PSR B1913 + 16. The energy loss of this system is well explained by emission of GWs. For this result, R.A. Hulse and J. H. Taylor were awarded the Physics Nobel Prize in 1993. Similar results were later confirmed in others relativistic binary systems. The precise measurements of the orbital parameters of this and other binary systems like PSR J0348 + 0432 make them very good laboratories for testing gravity in the limit of strong field, and one of the most promising tools. The best measured Post-Keplerian parameter is the time derivative of the orbital period $\dot{P}_{b}$ (Hulse and Taylor (1975), Damour and Esposito-Farèse (1998), Weisberg et al. (2010), Antoniadis et al. (2013)). Measurements of the Hulse-Taylor showed a discrepancy at the $\sim 1 \%$ with respect to the expected value (Hulse and Taylor (1975), Weisberg et al. (2010)). The discrepancy could be explained by astrophysical effects that can complicate the evolution of those systems like mass transfer between the components. In addition, there are several kinematic effects that need to be considered. First, the Shklovskii effect, related to the proper motion of the binary stars. The transverse velocity of the system results in an increasing projected distance of the pulsar to the Solar System barycenter, which affects any observed change in periodicities in the system (Shklovskii, 1973). The variation of the period due to this effect is expressed as

$$
\dot{P}_{\mathrm{b}}^{\mathrm{Shk}}=P_{\mathrm{b}} \frac{\mu^{2} d}{c},
$$

where $P_{\mathrm{b}}$ is the orbital period around the center of mass, $\mu$ is the reduced mass and $d$ is the distance to the Solar System. Second, the difference of the Galactic accelerations between the Sun and the system gives an apparent period increment

$$
\dot{P}_{\mathrm{b}}^{\mathrm{Acc}}=P_{\mathrm{b}} \frac{a_{c}}{c},
$$

where $a_{c}$ is the acceleration of the binary system around the galactic center. Finally, 
if the gravitational constant $\dot{G}$ varies in time, it will appear as a change in the pulsar period (Damour and Taylor (1991), Damour et al. (1988), Hofmann et al. (2010), Nordtvedt (1990)):

$$
\dot{P}_{\mathrm{b}}^{\dot{G}}=-2 P_{b} \frac{\dot{G}}{G} .
$$

Nevertheless, the discrepancies could also be understood using a different formulation of gravity (Freire et al., 2012). In this Chapter we will study the variation of the orbital period in an analytic $f(R)$ theory of gravity compared with GR. We will evaluate the first time derivative of the orbital and compare the predictions for a sample of binary systems. As a result of this study, we will set up bounds on the parameters of the theory and on the graviton mass (De Laurentis and De Martino (2013a,b)).

\subsection{Gravitational Waves: general framework and emission from binary systems}

Binary systems of collapsed objects are optimal laboratories to test gravity in the strong field limit (Damour and Esposito-Farèse (1998)) as opposed to solar system tests that constrain gravity at the same scales but in the weak field limit. Such systems could be composed of two compact objects such as Neutron Stars (NSs), White Dwarfs (WDs) or Black Holes (BHs). During the gradual evolution of inspiraling coalescing binaries the system loses energy and angular momentum, causing a shrinking of the orbit (Hulse and Taylor (1975), Weisberg and Taylor (2002),Nice and Thorsett (2003), Stairs (2004)). Nevertheless, the system also loses linear momentum as first suggested by Bekestein (1973) which computed the formula of the emission flux of linear momentum in linearized gravity showing that it is a quadrupole-octupole effect. The flux of linear momentum carried out by the GWs emission is of lower order than that of the energy and angular momentum.

The emission of GWs is described in the weak field limit. The space-time metric is assumed to deviate only slightly from the Minkowskian metric: $g_{\mu \nu}=\eta_{\mu \nu}+h_{\mu \nu}$ (for more details see Maggiore (2007), and Weinberg (1972)). A linear approximation is first order in $h_{\mu \nu}$ and eqs. (1.4), (1.5) can be written as

$$
\begin{aligned}
& \Gamma_{\mu \nu}^{\alpha}=\frac{1}{2} \eta^{\alpha \lambda}\left(\partial_{\nu} h_{\lambda \mu}+\partial_{\mu} h_{\lambda \nu}-\partial_{\lambda} h_{\mu \nu}\right), \\
& R_{\mu \nu \beta \alpha}=\frac{1}{2}\left(\partial_{\beta \nu} h_{\mu \alpha}+\partial_{\alpha \mu} h_{\nu \beta}-\partial_{\beta \mu} h_{\nu \alpha}-\partial_{\alpha \nu} h_{\mu \beta}\right) .
\end{aligned}
$$

Let us remark that eq. (3.5) is invariant under the transformation $h_{\mu \nu} \rightarrow h_{\mu \nu}-\partial_{\mu} \xi_{\nu}-$ $\partial_{\nu} \xi_{\mu}$. Before writing the field equations, we introduce the trace-reverse tensor

$$
\bar{h}^{\mu \nu}=h^{\mu \nu}-\frac{1}{2} \eta^{\mu \nu} h
$$


with $h=\eta_{\alpha \beta} h^{\alpha \beta}$ and $\bar{h}=-h$ (what explains the name). After a straightforward calculation we obtain

$$
\square \bar{h}_{\mu \nu}+\eta_{\mu \nu} \partial^{\rho} \partial^{\lambda} \bar{h}_{\rho \lambda}-\partial^{\rho} \partial_{\mu} \bar{h}_{\rho \nu}-\partial^{\rho} \partial_{\nu} \bar{h}_{\rho \mu}+\mathcal{O}\left(h^{2}\right)=-\frac{16 \pi G}{c^{4}} T_{\mu \nu},
$$

where the d'Alembertian operator is defined as $\square=\eta_{\rho \sigma} \partial^{\rho} \partial^{\sigma}$. The equations simplify in the Lorentz gauge (also known as harmonic gauge)

$$
\partial_{\nu} \bar{h}^{\mu \nu}=0
$$

Then, eqs. (3.7) become

$$
\square \bar{h}_{\mu \nu}=-\frac{16 \pi G}{c^{4}} T_{\mu \nu} .
$$

By choosing a gauge, the metric tensor $h_{\mu \nu}$ only contains six independent components. The equations for the vacuum $\left(T_{\mu \nu}=0\right)$ are

$$
\square \bar{h}_{\mu \nu}=0
$$

In the Lorentz one can set $\bar{h}_{\mu \nu}=h_{i j}^{\mathrm{TT}}$, where $h_{i j}^{\mathrm{TT}}$ is the transverse-traceless tensor satisfying the following relations

$$
h^{00}=0, \quad h^{0 i}=0, \quad \partial_{i} h^{i j}=0, \quad h^{i i}=0 .
$$

If the wave propagates along the $z$-axis, the traceless-transverse tensor can be written as

$$
h_{i j}^{\mathrm{TT}}(t, z)=\left(\begin{array}{ccc}
h_{+} & h_{\times} & 0 \\
h_{\times} & -h_{+} & 0 \\
0 & 0 & 0
\end{array}\right) \cos \left[\omega\left(t-\frac{z}{c}\right)\right],
$$

where $h_{+}$and $h_{\times}$denote the two independent polarization modes. The two modes, plus $(+)$ and cross $(\times)$, differ by a rotation of $45^{\circ}$. Fig. 3.1 represents the motion of point-like particles distributed on a ring due to a GW propagating perpendicularly to the plane of the ring. The solid lines represent the force lines associated to the two polarization modes.

For an observer at a very large distance, compared with the size of the binary system, the Gravitational Wave energy flux emitted per unit area is

$$
\frac{d E}{d t d A}=\frac{c^{3}}{16 \pi G}\left\langle\dot{h}_{+}^{2}+\dot{h}_{\times}^{2}\right\rangle .
$$

The energy emitted in the form of gravitational waves can be very significant. During a Supernovae event, a Gravitational Wave burst lasting a few ms has a luminosity of $d E /(d t) \sim 10^{52} \mathrm{erg} / \mathrm{s}$. For comparison, the neutrino luminosity is $\sim 10^{53} \mathrm{erg} / \mathrm{s}$ and the photon emission in optical band is $\sim 10^{43} \mathrm{erg} / \mathrm{s}$, radiated during periods that can vary from few seconds to about a week, respectively. 


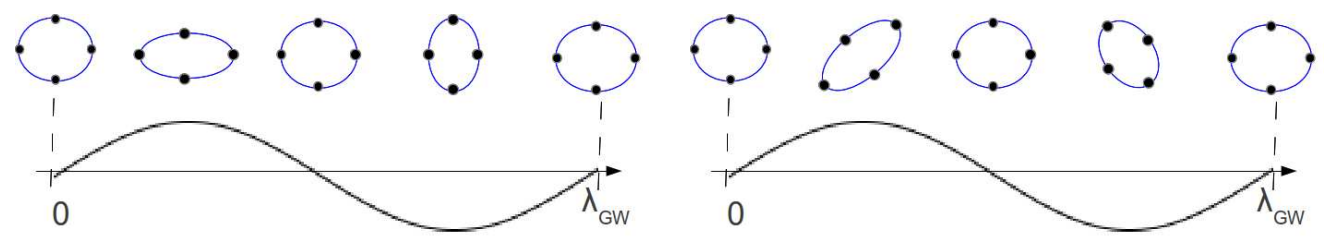

Figure 3.1: Motion of point particles distributed on ring as due to the interaction with a GW that propagates perpendicular to the plane of the ring and the lines of the force generated by the wave. The left panel represents a wave with polarization $(+)$ and the right panel with polarization $(\times)$.

If the internal motions of the binary system are non-relativistic, simple solutions of eq. (3.9) can be derived neglecting the self-gravity of the system. Under these simplifying assumptions, the transverse-traceless tensor is

$$
h_{i j}^{\mathrm{TT}}(t, \mathbf{x})=\frac{1}{r} \frac{2 G}{c^{4}} \Lambda_{i j, k l}(\mathbf{n}) \ddot{M}^{k l}\left(t-\frac{r}{c}\right),
$$

where $r$ is the distance from the source to the observer. The tensor $\Lambda_{i j, k l}(\mathbf{n})$ is related to the projector operator $P_{i j}=\delta_{i j}-n_{i} n_{j}$ as

$$
\Lambda_{i j, k l}=P_{i k} P_{j l}-\frac{1}{2} P_{i j} P_{k l}
$$

and $M^{k l}$ are the momenta of the mass density defined as follows ${ }^{1}$ (Maggiore, 2007)

$$
\begin{aligned}
M & =\frac{1}{c^{2}} \int d^{3} x T^{00}(t, \mathbf{x}) \\
M^{i} & =\frac{1}{c^{2}} \int d^{3} x T^{00}(t, \mathbf{x}) x^{i}, \\
M^{i j} & =\frac{1}{c^{2}} \int d^{3} x T^{00}(t, \mathbf{x}) x^{i} x^{j}
\end{aligned}
$$

If GWs propagate along the direction $\mathbf{n}=(\cos \phi, \sin \theta, \sin \phi, \sin \theta, \cos \theta)$ then from eq. (3.14) we obtain

$$
\begin{aligned}
h_{+}= & \frac{G}{r c^{4}}\left\{\ddot{M}_{11}\left(\sin ^{2} \phi-\cos ^{2} \theta \cos ^{2} \phi\right)+\ddot{M}_{22}\left(\cos ^{2} \phi-\cos ^{2} \theta \sin ^{2} \phi\right)-\ddot{M}_{33} \sin ^{2} \theta-\right. \\
& \left.-\ddot{M}_{12} \sin 2 \phi\left(1+\cos ^{2} \theta\right)+\ddot{M}_{13} \cos \phi \sin 2 \theta+\ddot{M}_{23} \sin 2 \theta \sin \phi\right\}
\end{aligned}
$$

and

$$
\begin{aligned}
h_{\times}= & \frac{2 G}{r c^{4}}\left\{\frac{1}{2}\left(\ddot{M}_{11}-\ddot{M}_{22}\right) \cos \theta \sin 2 \phi-\ddot{M}_{12} \cos \theta \cos 2 \phi-\ddot{M}_{13} \sin \theta \sin \phi+\right. \\
& \left.+\ddot{M}_{23} \cos \phi \sin \theta\right\} .
\end{aligned}
$$

${ }^{1}$ From the conservation law $\partial_{\mu} T^{\mu \nu}=0$, setting $\nu=0$, one can obtain the relation $\partial_{0} T^{00}+\partial_{i} T^{i 0}=$ 0. The integration over a volume containing the source yields the conservation of the mass $\dot{M}=0$. Similarly, one can derive the conservation of the momentum $\ddot{M}^{i}=0$ 
Using the eq (3.13), the power radiated in the form of GWs is

$$
\frac{d E}{d \Omega d t}=\frac{r^{2} c^{3}}{32 \pi G}\left\langle\dot{h}_{i j}^{\mathrm{TT}} \dot{h}_{i j}^{\mathrm{TT}}\right\rangle=\frac{G}{8 \pi c^{5}} \Lambda_{k l, m p}(\mathbf{n})\left\langle\dddot{Q}_{k l} \dddot{Q}_{m p}\right\rangle,
$$

where the traceless quadrupole tensor $Q_{i j}$ is defined as

$$
Q_{i j}=M_{i j}-\frac{1}{3} \delta_{i j} M_{k k}
$$

Taking into account that

$$
\int \frac{d \Omega}{4 \pi} n_{i} n_{j}=\frac{1}{3} \delta_{i j}
$$

and

$$
\int \frac{d \Omega}{4 \pi} n_{i} n_{j} n_{k} n_{l}=\frac{1}{15}\left(\delta_{i j} \delta_{k l}+\delta_{i k} \delta_{j l}+\delta_{i l} \delta_{j k}\right),
$$

the total radiated power is

$$
\frac{d E}{d t}=\frac{G}{5 c^{5}}\left\langle\dddot{Q}_{i j} \dddot{Q}_{i j}\right\rangle,
$$

or equivalently, in terms of the mass-density momentum,

$$
\frac{d E}{d t}=\frac{G}{5 c^{5}}\left\langle\dddot{M}_{i j} \dddot{M}_{i j}-\frac{1}{3}\left(\dddot{M}_{k} k\right)^{2}\right\rangle .
$$

The formalism reviewed above is fully general and can be applied to any source of GWs. Next, we will particularize it for binary systems.

\subsubsection{Application to binary systems}

At present, binary systems of collapsed objects provide the best evidence of the GW emission. In this subsection we will summarize the main theoretical aspects. To simplify the presentation, let us assume that the orbits of both stars are Keplerian and they move on the $(x, y)$-plane. Let us denote by $m_{p}$ the mass of the pulsar, $m_{c}$ of its companion and $\mu=\frac{m_{c} m_{p}}{m_{c}+m_{p}}$ the reduced mass of the system. In the center-of-mass frame, the relative coordinates are

$$
x(t)=r(t) \cos \psi, \quad y(t)=r(t) \sin \psi, \quad z(t)=0,
$$

and the radial distance between the two bodies evolves as

$$
r(t)=\frac{a\left(1-\epsilon^{2}\right)}{1+\epsilon \cos (\psi(t))}
$$

where $\psi$ is the eccentric anomaly. Both magnitudes depend on time. The only nonzero components of the mass-momentum are in the plane of the motion and can be written as

$$
M_{i j}=\mu r^{2}\left(\begin{array}{cc}
\cos ^{2} \psi & \sin \psi \cos \psi \\
\sin \psi \cos \psi & \sin ^{2} \psi
\end{array}\right)_{i j}
$$


where $i, j=(x, y)$ are the indexes in the orbital plane. To compute the radiated power one is required to compute the third derivative of the quadrupole. The calculation is simplified by using the following relation (Maggiore, 2007)

$$
\dot{\psi}=\left(\frac{G m_{c}}{a^{3}}\right)^{\frac{1}{2}}\left(1-\epsilon^{2}\right)^{-\frac{3}{2}}(1+\epsilon \cos \psi)^{2}
$$

where $a$ is the semi-major-axis, and $\epsilon$ the eccentricity of the orbit. Defining the quantity

$$
\mathcal{H}_{1}=\frac{(2 \pi)^{5 / 3} G^{2 / 3} m_{c} m_{p}}{P_{b}^{5 / 3}\left(1-\epsilon^{2}\right)^{5 / 2}}
$$

the only third derivatives of the quadrupole different from zero are

$$
\begin{aligned}
& \dddot{M}_{11}=\mathcal{H}_{1} \sin 2 \psi(\epsilon \cos \psi+1)^{2}(3 \epsilon \cos \psi+4), \\
& \left.\dddot{M}_{22}=-\mathcal{H}_{1} \epsilon(3 \cos 2 \psi+5)+8 \cos \psi\right) \sin \psi(\epsilon \cos \psi+1)^{2}, \\
& \dddot{M}_{12}=-\mathcal{H}_{1}(\epsilon \cos \psi+1)^{2}(5 \epsilon \cos \psi+3 \epsilon \cos 3 \psi+8 \cos 2 \psi) .
\end{aligned}
$$

The average radiated power on an orbital period is

$$
\frac{d E}{d t}=\frac{1}{P_{b}} \int_{0}^{P_{b}} d t \frac{d E(\psi)}{d t}=\frac{1}{P_{b}} \int_{0}^{2 \pi} \frac{d \psi}{\dot{\psi}} \frac{d E(\psi)}{d t}
$$

and since $\frac{\dot{P}_{b}}{P_{b}}=-\frac{3}{2} \frac{\dot{E}}{E}$, the time variation of the orbital period becomes

$$
\dot{P}_{b}=-\frac{3}{15}\left(\frac{P_{b}}{2 \pi}\right)^{-\frac{5}{3}} \frac{\mu G^{\frac{5}{3}}\left(m_{c}+m_{p}\right)^{\frac{2}{3}}}{c^{5}\left(1-\epsilon^{2}\right)^{\frac{7}{2}}}\left(37 \epsilon^{4}+292 \epsilon^{2}+96\right) .
$$

The time derivative of the orbital period is the best determined Post-Keplerian parameter. Consequently, this magnitude provides the best constraints on theories of gravitation (Damour and Esposito-Farèse, 1998).

\subsection{Energy-momentum tensor and quadrupolar emission in $f(R)$-gravity}

To compute GWs emission in $f(R)$ we will follow the derivation of the previous section. By considering the field equations (1.75) and (1.76) in presence of matter, and using the convention on the signature $(+,-,-,-)$, it is possible compute the energy momentum tensor of gravitational field in $f(R)$-gravity. Adopting the definition given in Landau and Lifshitz (1962) and De Laurentis and Capozziello (2011), and imposing that it satisfies a conservation law as required by the Bianchi identities, ones can write

$$
t_{\alpha}^{\lambda}=\frac{1}{\sqrt{-g}}\left[\left(\frac{\partial \mathcal{L}}{\partial g_{\rho \sigma, \lambda}}-\partial_{\xi} \frac{\partial \mathcal{L}}{\partial g_{\rho \sigma, \lambda \xi}}\right) g_{\rho \sigma, \alpha}+\frac{\partial \mathcal{L}}{\partial g_{\rho \sigma, \lambda \xi}} g_{\rho \sigma, \xi \alpha}-\delta_{\alpha}^{\lambda} \mathcal{L}\right] .
$$


In GR, the energy radiated by a source is determined by the the time variation of the quadrupole. At each time the flux of energy loss by the system through a surface of area $r^{2} d \Omega$ in the direction $\hat{x}$ will be

$$
\frac{d E}{d t}=r^{2} \int d \Omega \hat{x}^{i} t^{0 i}
$$

Averaging over the period, the eq. (3.38) reads

$$
\left\langle\frac{d E}{d t}\right\rangle=r^{2} \int d \Omega \hat{x}^{i}\left\langle t^{0 i}\right\rangle
$$

In order to express the eq. (3.39) in term of the quadrupole emission, as in GR (Maggiore, 2007), one defines the momenta of mass-energy distribution using eqs. (3.16), (3.17), (3.18). Analyzing the radiation in terms of multipoles, De Laurentis and Capozziello (2011) have expressed the energy-momentum tensor in term of multipoles and, after integrating over all directions, in terms of the quadrupolar emission (for details, see Appendix D). The result is

$$
\underbrace{\left\langle\frac{d E}{d t}\right\rangle}_{\text {(total })}=\frac{G}{60 c^{5}}\langle\underbrace{f_{0}^{\prime}\left(\dddot{Q}^{i j} \dddot{Q}_{i j}\right)}_{\mathrm{GR}}-\underbrace{f_{0}^{\prime \prime}\left(\dddot{Q}^{i j} \dddot{Q}_{i j}\right)}_{f(R)}\rangle .
$$

In the limit $f_{0}^{\prime \prime} \rightarrow 0$ and $f_{0}^{\prime} \rightarrow \frac{4}{3}$, eq. (3.40) becomes

$$
\underbrace{\left\langle\frac{d E}{d t}\right\rangle}_{\mathrm{GR}}=\frac{G}{45 c^{5}}\left\langle\dddot{Q}^{i j} \dddot{Q}_{i j}\right\rangle
$$

recovering the relativistic result.

\subsection{First time derivative of the orbital period in $f(R)$ - gravity}

To make concrete prediction, we follow the GR case (Sect. 3.1.1). The power radiated in the form of GWs is given by eq. (3.40). Therefore, using the quadrupole matrix, given by eq. (3.29), the radius of the orbit (eq. (3.28)) and its eccentric anomaly (eq. (3.30)), we computed the time derivatives of degrees three and four in eq. (3.40)

$$
\begin{aligned}
& \dddot{Q}_{11}=\mathcal{H}_{1} \sin 2 \psi(\epsilon \cos \psi+1)^{2}(3 \epsilon \cos \psi+4), \\
& \dddot{Q}_{22}=-\mathcal{H}_{1}(8 \cos \psi+\epsilon(3 \cos 2 \psi+5)) \times \sin \psi(\epsilon \cos \psi+1)^{2}, \\
& \dddot{Q}_{12}=-\mathcal{H}_{1}(\epsilon \cos \psi+1)^{2} \times(5 \epsilon \cos \psi+3 \epsilon \cos 3 \psi+8 \cos 2 \psi),
\end{aligned}
$$


and

$$
\begin{aligned}
& \dddot{Q}_{11}=\mathcal{H}_{2}\left[15 \epsilon^{2} \cos 4 \psi+50 \epsilon \cos 3 \psi+\left(12 \epsilon^{2}+32\right) \cos 2 \psi+6 \epsilon \cos \psi-3 \epsilon^{2}\right], \\
& \dddot{Q}_{22}=-\mathcal{H}_{2}\left[15 \epsilon^{2} \cos 4 \psi+50 \epsilon \cos 3 \psi+\left(24 \epsilon^{2}+32\right) \cos 2 \psi+14 \epsilon \cos \psi-7 \epsilon^{2}\right], \\
& \dddot{Q}_{12}=2 \mathcal{H}_{2} \sin \psi\left[15 \epsilon^{2} \cos 3 \psi+50 \epsilon \cos 2 \psi+\left(33 \epsilon^{2}+32\right) \cos \psi+30 \epsilon\right]
\end{aligned}
$$

where we have defined $\mathcal{H}_{1}$ as given in eq. (3.31) and $\mathcal{H}_{2}$ as

$$
\mathcal{H}_{2}=\frac{2^{2 / 3} \pi^{8 / 3} G^{2 / 3} m_{c} m_{p}(\epsilon \cos \psi+1)^{3}}{P_{b}^{8 / 3}\left(\epsilon^{2}-1\right)^{4} \sqrt[3]{m_{c}+m_{p}}} .
$$

Taken the average radiated power over an orbital period and with eq. (3.40) and, again, the relation $\frac{\dot{P}_{b}}{P_{b}}=-\frac{3}{2} \frac{\dot{E}}{E}$, the time variation of the orbital period is

$$
\begin{aligned}
\dot{P}_{b}= & -\frac{3}{20}\left(\frac{P_{b}}{2 \pi}\right)^{-\frac{5}{3}} \frac{\mu G^{\frac{5}{3}}\left(m_{c}+m_{p}\right)^{\frac{2}{3}}}{c^{5}\left(1-\epsilon^{2}\right)^{\frac{7}{2}}} \times\left[f_{0}^{\prime}\left(37 \epsilon^{4}+292 \epsilon^{2}+96\right)-\frac{f_{0}^{\prime \prime} \pi^{2} P_{b}^{-1}}{2\left(1+\epsilon^{2}\right)^{3}} \times\right. \\
& \left.\times\left(891 \epsilon^{8}+28016 \epsilon^{6}+82736 \epsilon^{4}+43520 \epsilon^{2}+3072\right)\right] .
\end{aligned}
$$

Before concluding, let us remarks that if we set $f_{0}^{\prime \prime}=0$, (that is $f(R)=R$ ) we recover the GR result of eq. (3.36). In the next section we will constrain this parameter using observations.

\subsection{Constraints on ETGs from binary systems}

The time derivative of the orbital period $\dot{P}_{b}$ is the best measured orbital parameter and it is the one that offers the strongest constraints on theories since can be directly related to the parameters of the theory. The normal approach would be to assume the Lagrangian of our theory and predict the energy loss through gravitational waves, i.e., particularize eq. (3.49) for any given Lagrangian. We are going to take the inverse approach. We will use the data to constraint $f(R)$-parameters and reconstruct the Lagrangian. We will assume that the difference between the variation of the observed binary period $\dot{P}_{b_{O b s}}$ and the GR prediction is a consequence of GR not being the correct theory of gravity, then the difference would be the $f(R)$ correction

$$
\dot{P}_{b_{O b s}}-\dot{P}_{G R}=f_{0}^{\prime \prime} \dot{P}_{b_{f(R)}} \text {. }
$$

We will not look for alternative explanations based on astrophysical phenomena like mass transfer between the systems, stellar winds and other complications. In this 
context, the uncertainty $\delta$ of the measured period will translates into an uncertainty on second derivative of gravitational theory, $\sigma_{f_{0}^{\prime \prime}}$ as

$$
\dot{P}_{b_{O b s}} \pm \delta-\dot{P}_{G R}=\left(f_{0}^{\prime \prime} \pm \sigma_{f_{0}^{\prime \prime}}\right) \dot{P}_{b_{f(R)}} .
$$

Isolating $f_{0}^{\prime \prime}$ and $f_{0_{ \pm \delta}}^{\prime \prime}$ from eqs. (3.50) and (3.51) we obtain

$$
f_{0}^{\prime \prime}=\frac{\Delta \dot{P}_{b}}{\dot{P}_{b_{f(R)}}}
$$

and

$$
f_{0}^{\prime \prime} \pm \sigma_{f_{0}^{\prime \prime}}=\frac{\dot{P}_{b_{O b s}} \pm \delta-\dot{P}_{G R}}{\dot{P}_{b_{f(R)}}} .
$$

From public catalogs of binary systems we selected a sample of Observed Relativistic Binary Pulsars (ORBP) with well measured orbital parameters: period ( $P_{b_{O b s}}$, in days), its time variation $\left(\dot{P}_{b_{O b s}}\right)$, experimental uncertainty $( \pm \delta)$, General Relativity $\left(\dot{P}_{G R}\right)$, projected semi-major axis $\operatorname{asin}(i)$ (in light seconds), orbital eccentricity $\epsilon$, mass of the two components $m_{p}, m_{c}$ (in solar masses). For those pulsars where the orbital decay due to the galactic or Shklovskii acceleration are available, we have subtracted these contributions. All the observed properties together with the GR prediction of the time derivative of the period $\left(\dot{P}_{G R}\right)$ are given in Table 3.1. For the first five systems the masses have been determined reliably while for the remaining sample, masses were estimated taking $m_{p}=1.4 M_{\odot}$ and assuming either $i=60^{\circ}$ or $i=90^{\circ}$. The last four columns show the results of our analysis. For each system in the Table, we give: $\Delta \dot{P}_{G R}$ (equal to $-f_{0}^{\prime \prime} \dot{P}_{b_{f(R)}}$, see $(3.52)$ ), $\dot{P}_{b_{f(R)}}, f_{0}^{\prime \prime}$ (given by (3.52)) and finally, the error on $f_{0}^{\prime \prime}$ and computed from the difference $\frac{\left(f_{0}^{\prime \prime}+\sigma_{f_{0}^{\prime \prime}}\right)-\left(f_{0}^{\prime \prime}-\sigma_{f_{0}^{\prime \prime}}\right)}{2}$. Our results show that for most binary systems the value of $f_{0}^{\prime \prime}$ is very different of zero (by many order of magnitudes). For those systems we can only conclude that external factors like mass transfer, uncertainties on orbital parameters or on the mass of the stars are large and need to be taken into account.

In Fig. 3.2 we show the $f(R)$ prediction of $\dot{P}_{b}$ for different values of $f_{0}^{\prime \prime}$ for two specific systems (solid black line), the measured period (blue line) and its corresponding error bars (red lines). The green line shows the GR prediction. As expected, the black and green lines cross at $f_{0}^{\prime \prime}=0$. The error bars reported in the last column in Table 3.1 are obtained from where the black line crosses the red lines. In the panel (a) for the system $\mathbf{J} \mathbf{2 1 2 9}+\mathbf{1 2 1 0} \mathbf{C}$ the GR prediction $\dot{P}_{G R}$ is compatible with the measured value. We point out the GR value of $\dot{P}_{G R}$ is recovered for $f^{\prime \prime}(r)=0$ (green square), while to justify the difference between $\dot{P}_{b_{O b s}}$ and $\dot{P}_{G R}$ we show the value of $f_{0}^{\prime \prime}$ (blue square) and its error band $f_{0}^{\prime \prime} \pm \sigma_{f_{0}^{\prime \prime}}$ (red square) as computed in eqs. (3.52) and (3.53). In the last panel (b) there are reported for $\mathbf{J 0 7 5 1 + 1 8 0 7}$ the same data but in this case the $\dot{P}_{G R}$ is $\mathbf{O U T}$ of the error band determined by the experimental errors $\pm \delta$. It is possible to 
Table 3.1: Observational data on binary pulsars. The columns, from left to write, are name, orbital period $T_{b}$ (in days), projected semi-major axis $a(\sin i)$ (in light seconds), eccentricity $\epsilon$, time variation of the period $\dot{P}_{O b s}$, GR prediction $\dot{P}_{G R}$, observational uncertainty $\pm \delta$ of $\dot{P}_{O b s}$ and mass of the components $m_{p}$ and $m_{c}$ (in solar masses). Then, there included our results: the difference between $\dot{P}_{b_{o b s}}$ and $\dot{T}_{G R}$, the correction $\dot{P}_{b_{f(R)}}, f_{0}^{\prime \prime}$ derived from (3.50) shown in (3.52), error on $f_{0}^{\prime \prime}$.

\begin{tabular}{|c|c|c|c|c|c|c|c|c|c|c|c|c|c|}
\hline Name & $\begin{array}{c}T_{b} \\
\text { (days) }\end{array}$ & $\begin{array}{c}a \\
\text { (lsec) }\end{array}$ & $\begin{array}{c}i \\
\text { (degrees) }\end{array}$ & $\epsilon$ & $\begin{array}{c}\dot{P}_{b_{O b s}} \\
\left(10^{-12}\right)\end{array}$ & $\begin{array}{c}\dot{T}_{G R} \\
\left(10^{-12}\right)\end{array}$ & $\begin{array}{c} \pm \delta \\
\left(10^{-12}\right)\end{array}$ & $\begin{array}{c}m_{p} \\
\left(M_{\odot}\right)\end{array}$ & $\begin{array}{c}m_{c} \\
\left(M_{\odot}\right)\end{array}$ & $\begin{array}{c}\Delta \dot{P}_{G R} \\
\left(10^{-12}\right)\end{array}$ & $\begin{array}{c}\dot{P}_{b_{f(R)}} \\
\left(10^{-12}\right)\end{array}$ & $f_{0}^{\prime \prime}$ & $\sigma_{f_{0}^{\prime \prime}}$ \\
\hline $\bar{J} 2129+1210 \mathrm{C}^{*}$ & 0.335282049 & 2.51845 & - & 0.681395 & $\begin{array}{l}-3.96 \\
\end{array}$ & $\begin{array}{l}-3.94 \\
\end{array}$ & 0.05 & 1.358 & 1.354 & -0.0217 & 0.601 & 0.04 & 0.08 \\
\hline $\mathrm{J} 1915+1606^{+}$ & 0.322997449 & 2.341782 & $54.12^{\circ}$ & 0.6171334 & -2.423 & -2.403 & 0.001 & 1.4398 & 1.3886 & -0.0204 & 0.210 & 0.10 & 0.05 \\
\hline J0737-3039A $\#$ & 0.102251562 & 1.415032 & $88.69^{\circ}$ & 0.0877775 & -1.252 & -1.248 & 0.017 & 1.3381 & 1.2489 & -0.00423 & 0.0186 & 0.23 & 0.09 \\
\hline $\mathrm{J} 1141-6545^{\S}$ & 0.197650959 & 1.858922 & $73^{\circ}$ & 0.171884 & -0.403 & -0.387 & 0.025 & 1.27 & 1.02 & -0.0165 & 0.00388 & 4.25 & 6.44 \\
\hline $\mathrm{J} 1537+1155^{\nabla}$ & 0.420737299 & 3.7294626 & $78.4^{\circ}$ & 0.2736767 & -0.138 & -0.192 & 0.0001 & 1.3332 & 1.3452 & 0.0539 & 0.00142 & -37.90 & 0.07 \\
\hline $\mathrm{J} 1738+0333^{\diamond}$ & 0.3547907399 & 0.343429 & $32.6^{\circ}$ & $3.4 \mathrm{E}-7$ & -0.017 & -0.0277 & 0.0031 & 1.46 & 0.181 & -0.00156 & $1.06 \mathrm{E}-4$ & -14.7 & 29.2 \\
\hline $\mathrm{J} 0751+1807 \triangleleft$ & 0.263144267 & 0.3966127 & $65.8^{\circ}$ & $7.1 \mathrm{E}-7$ & -0.031 & -0.017 & 0.009 & 1.7 & 0.67 & 0.141 & $8.98 \mathrm{E}-4$ & -157.0 & 10.02 \\
\hline J0024-7204J ${ }^{\square}$ & 0.120664938 & 0.0404021 & $60^{\circ}$ & 0 & -0.55 & -0.03 & 0.13 & 1.4 & 0.024 & -0.522 & $3.13 \mathrm{E}-4$ & 1670 & 415 \\
\hline J1701-3006B ${ }^{\boldsymbol{\Lambda}}$ & 0.144545417 & 0.2527565 & $84.7^{\circ}$ & 0 & -5.12 & -0.09 & 0.062 & 1.4 & 0.14 & -5.03 & $8.81 \mathrm{E}-4$ & 5710 & 70.4 \\
\hline $\mathrm{J} 2051-0827^{\star}$ & 0.099110251 & 0.045052 & $30^{\circ}$ & 0 & -15.5 & -0.03 & 0.8 & 1.4 & 0.027 & -15.5 & $4.77 \mathrm{E}-4$ & $3.24 \mathrm{E}+4$ & $1.68 \mathrm{E}+3$ \\
\hline J1909-3744 & 1.533449475 & 1.8979910 & $86.4^{\circ}$ & $1.302 \mathrm{E}-07$ & -0.55 & -0.003 & 0.03 & 1.57 & 0.212 & -0.547 & $2.62 \mathrm{E}-6$ & $2.09 \mathrm{E}+05$ & $1.14 \mathrm{E}+04$ \\
\hline $\mathrm{J} 1518+4904^{\ddagger}$ & 8.634005096 & 20.044002 & $<47^{\circ}$ & 0.24948451 & 0.24 & -0.001 & 0.22 & 1.56 & 1.05 & 0.241 & $3.42 \mathrm{E}-7$ & $-7.05 \mathrm{E}+5$ & $6.43 \mathrm{E}+3$ \\
\hline $\mathrm{J} 1959+2048 \bullet$ & 0.381966607 & 0.0892253 & $65^{\circ}$ & 0 & 14.7 & -0.003 & 0.8 & 1.4 & 0.022 & 14.7 & $1.07 \mathrm{E}-5$ & $-1.38 \mathrm{E}+6$ & $7.51 \mathrm{E}+4$ \\
\hline $\mathrm{J} 2145-0750^{\text {枯 }}$ & 6.83893 & 10.164108 & - & 0.0000193 & 0.4 & -0.0005 & 0.3 & 1.4 & 0.5 & 40.1 & $1.00 \mathrm{E}-7$ & $-4.00 \mathrm{E}+6$ & $2.99 \mathrm{E}+6$ \\
\hline $\mathrm{J} 0437-4715^{\oplus}$ & 5.74104646 & 3.36669708 & $137.58^{\circ}$ & 0.00001918 & 0.159 & -0.0004 & 0.283 & 1.76 & 0.254 & 15.9 & $1.04 \mathrm{E}-7$ & $-1.57 \mathrm{E}+6$ & $2.73 \mathrm{E}+6$ \\
\hline J0045-7319 ${ }^{\circledast}$ & 51.169451 & 174.2576 & $44^{\circ}$ & 0.807949 & $-3.03 \mathrm{E}+5$ & -0.02242 & $9 \mathrm{E}+3$ & 1.4 & 8.8 & $3.02 \mathrm{E}-05$ & $1.11 \mathrm{E}-4$ & $2.74 \mathrm{E}+9$ & $8.13 \mathrm{E}+7$ \\
\hline $\mathrm{J} 2019+2425^{\star}$ & 76.51163479 & 38.7676297 & $63^{\circ}$ & 0.00011109 & -30.0 & -0.000006 & 60.0 & 1.33 & 0.35 & -30.0 & $1.11 \mathrm{E}-10$ & $2.71 \mathrm{E}+11$ & $5.41 \mathrm{E}+11$ \\
\hline $\mathrm{J} 1623-2631^{*}$ & 191.44281 & 64.80946 & $40^{\circ}$ & 0.02531545 & 400.0 & -0.000003 & 600.0 & 1.3 & 0.8 & $4.00 \mathrm{E}-8$ & $2.02 \mathrm{E}-11$ & $-1.98 \mathrm{E}+13$ & $2.97 \mathrm{E}+13$ \\
\hline \multicolumn{14}{|c|}{ Anderson et al. (1990), Jacoby et al. (2006) } \\
\hline "Burgay et al. (2 & 3), Kramer et al. & 2006) & \multirow{2}{*}{\multicolumn{11}{|c|}{ Kaspi et al. (2000), Bhat et al. (2008) }} \\
\hline \multicolumn{3}{|c|}{$\begin{array}{l}\text { Burgay et al. (2003), Kramer et al. (2006) } \\
\nabla \text { Stairs et al. (2002), Konacki et al. (2003) }\end{array}$} & & & & & & & & & & & \\
\hline \multicolumn{3}{|c|}{ Lundgren et al. (1995), Nice et al. (2008) } & \multicolumn{11}{|c|}{$\begin{array}{l}\diamond \text { Freire et al. (2012) } \\
\text { Freire et al. (2003). Camilo et al. (2000) }\end{array}$} \\
\hline \multirow{2}{*}{\multicolumn{3}{|c|}{$\begin{array}{l}\triangle \text { Possenti et al. (2003), Lynch et al. (2012) } \\
\text { Jacoby et al. (2003), Verbiest et al. (2009) }\end{array}$}} & \multicolumn{11}{|c|}{ * Stappers et al. (1996), Doroshenko et al. (2001) } \\
\hline & & & \multirow{2}{*}{\multicolumn{11}{|c|}{$\begin{array}{l}\text { Nice et al. (1996), Janssen et al. (2008) } \\
\text { Bailes et al. (1994), Verbiest et al. }\end{array}$}} \\
\hline \multirow{2}{*}{\multicolumn{3}{|c|}{$\begin{array}{l}\text { - Fruchter et al. (1988), Arzoumanian et al. (1994) } \\
\text { Johnston et al. (1993), Verbiest et al. (2008) }\end{array}$}} & & & & & & & & & & & \\
\hline & & & $\circledast$ McConnel & et al. (1991), K & spi et al. (199 & & & & & & & & \\
\hline \multicolumn{3}{|c|}{ - Nice et al. $(1993,2001)$} & \multicolumn{11}{|c|}{ - Lyne et al. (1988), Thorsett et al. (1999) } \\
\hline
\end{tabular}


see for $f_{0}^{\prime \prime}=0$ that the GR value of $\dot{P}_{G R}$ is recovered, but in this case the $f_{0}^{\prime \prime}$ values are much greater than the previous ones. Let us remark that both systems predict values of $f_{0}^{\prime \prime}$ different by orders of magnitude. Therefore, systems like $J 0751+1807$ are very difficult to accommodate in the ETGs framework. For these system we need a more accurate analysis of systematics and physical processes that can altered the measured period.

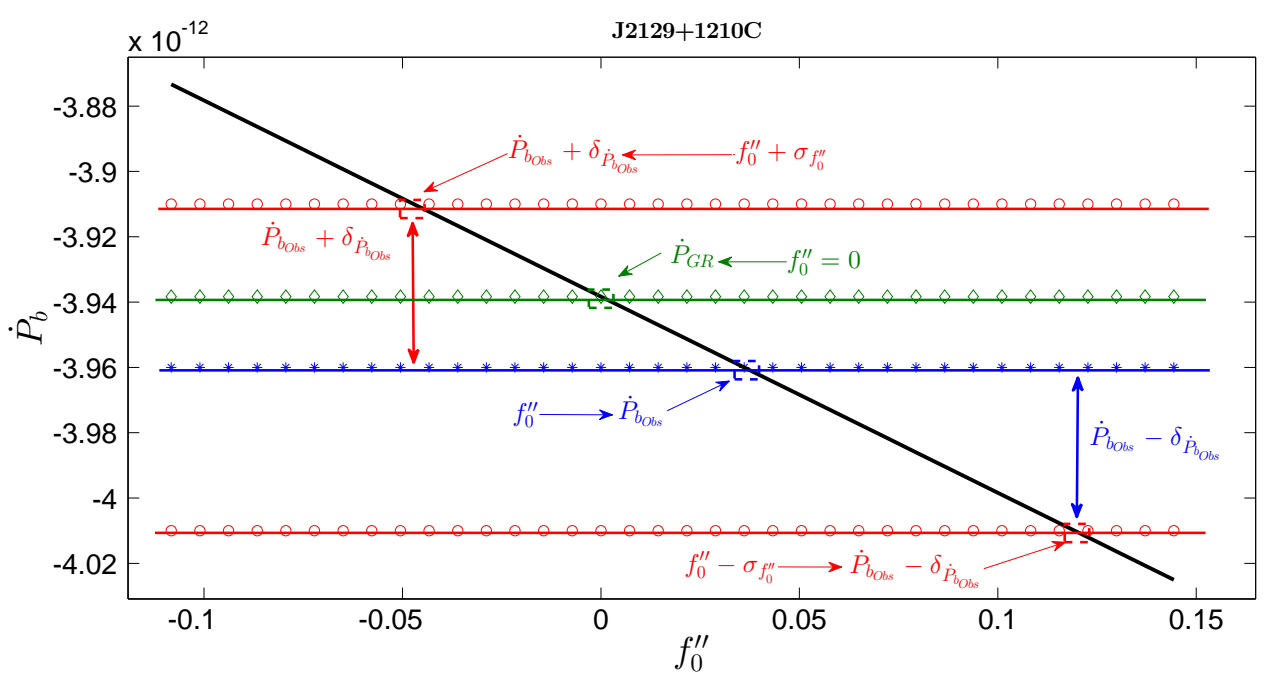

(a)

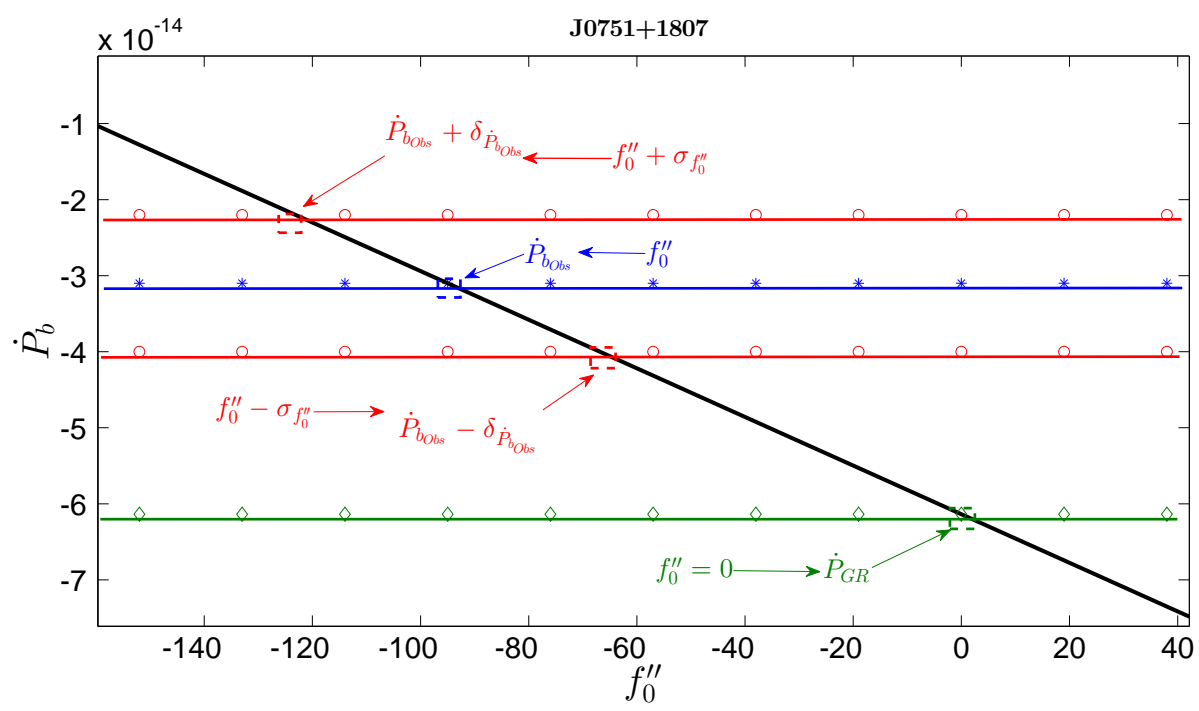

(b)

Figure 3.2: Time variation of the orbital period as a function of $f_{0}^{\prime \prime}$ (solid black line) measured values (blue line) and observational uncertainties (red lines). The green line represents the GR prediction. 


\subsection{Massive or massless gravitons?}

In Extended Theories of Gravity, higher-order terms in the Lagrangian or the induced scalar fields give rise to massive gravitons in a natural way (Bogdanos et al., 2010), adding extra polarization modes that could potentially be detected on CMB temperature anisotropies. The Yukawa correction in the post Newtonian regime depends on the characteristic length of the self-gravitating system $L$ (see Sect. 1.7.2) that is related to the mass of these gravitational modes. Basically, the mass of the graviton is given by eq. (1.63) that, by using eqs. (1.81) and (1.83), can be recast as

$$
m_{g}=\sqrt{3} / L
$$

Therefore, upper limits on the mass of the graviton can be obtained from constraints on the value of $L$.

\subsubsection{An upper limit on the graviton mass from PSR $J 0348+0432$.}

The binary pulsar PSR $J 0348+0432$ is composed by a pulsar spinning at $39 \mathrm{~ms}$ with mass $m_{p}=2.01 \pm 0.04 M_{\odot}$ and a WD companion with mass $m_{c}=0.172 \pm 0.003 M_{\odot}$. Since the masses of the system are very well estimated, and the high mass of the pulsar provide a good test bed of ETGs and provide upper limits on the mass of the graviton (Antoniadis et al., 2013). All the parameters of this system are given in Table 3.2. The intrinsic variation of the orbital period, after subtracting the kinematic effects, is $\dot{P}_{b}=(-2.73 \pm 0.45) \times 10^{-13}$.

\begin{tabular}{|l|c|}
\hline \hline Name & Value \\
\hline \hline Pulsar Mass, $m_{p}$ & $2.01 \pm 0.04 M_{\odot}$ \\
Companion Mass, $m_{c}$ & $0.172 \pm 0.003 M_{\odot}$ \\
Orbital period, $P_{b}$ (days) & 0.102 \\
eccentricity, $e$ & $2.36008 \times 10^{-6}$ \\
Shklovskii effect, $\dot{P}_{\mathrm{b}}^{\text {Shk }}$ & $0.0129_{-0.0021}^{+0.0025} \times 10^{-13}$ \\
Galactic Acceleration, $\dot{P}_{\mathrm{b}}^{\text {Acc }}$ & $0.0037_{-0.0005}^{+0.0006} \times 10^{-13}$ \\
Gravitational Term, $\dot{P}_{\mathrm{b}}^{\dot{G}}$ & $(0.0003 \pm 0.0018) \times 10^{-13}$ \\
Orbital Decay, $\dot{P}_{b}$ & $(-2.73 \pm 0.45) \times 10^{-13}$ \\
\hline \hline
\end{tabular}

Table 3.2: PSR $J 0348+0432$ : orbital parameters and kinematic contributions to the orbital decay.

Fig. 3.3 shows the intersection of $\dot{P}_{b}$ with the measured value and its uncertainties of PSR $J 0348+0432$ for different values of $f_{0}^{\prime \prime}$. solid black, blue and red lines and the red dashed lines correspond to the GR prediction, the $f(R)$ prediction, the observed orbital period and its uncertainties, respectively. The result is $f_{0}^{\prime \prime}=4.02 \pm 5.48$; the 
central value is represented by a blue triangle fully comparable with the range given in De Laurentis and De Martino (2013a), and compatible with zero.

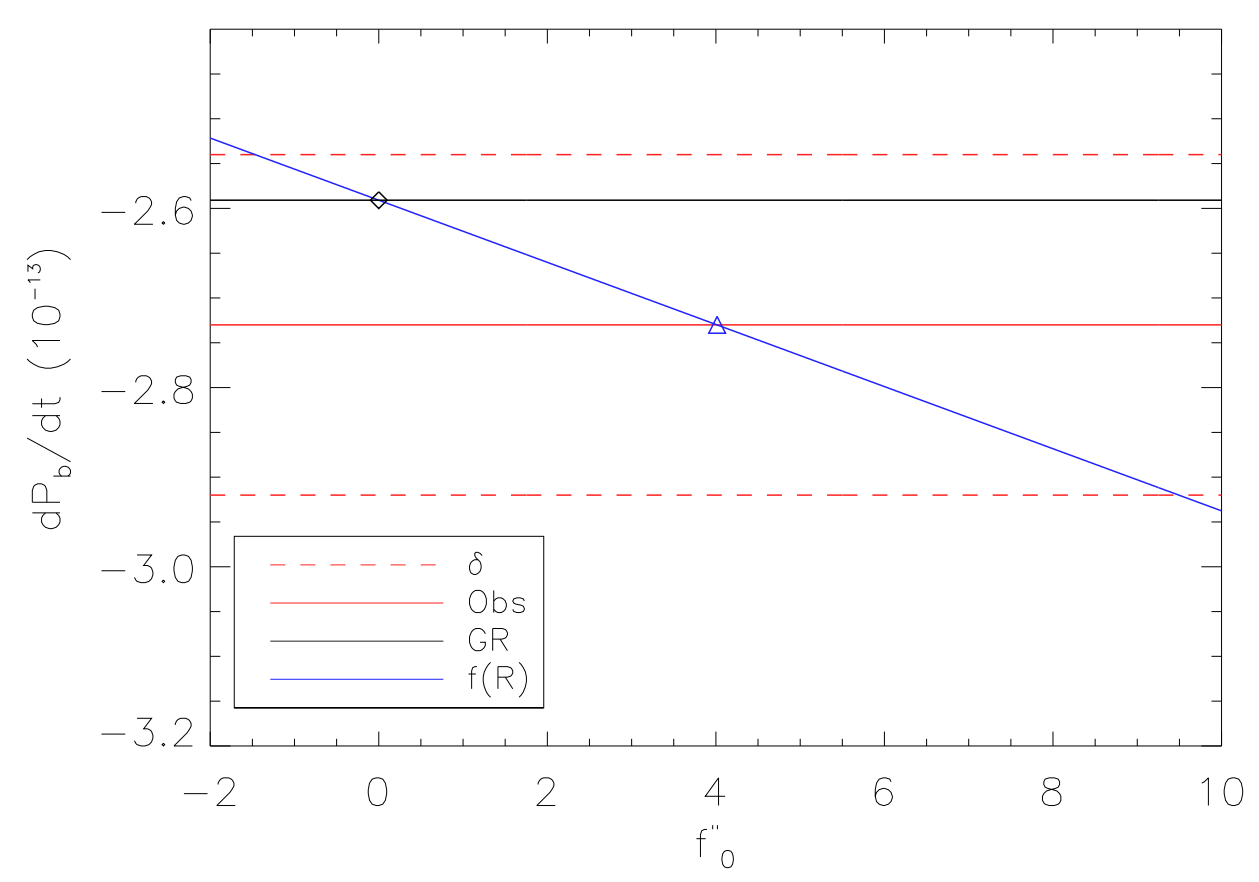

Figure 3.3: Analysis of the binary system PSR $J 0348+0432$.

From the range of the allowed values of $f_{0}^{\prime \prime}$ we can compute an upper limit on the graviton mass. Following Finn and Sutton (2002), the upper limit on the graviton mass is related to the difference between theoretical prediction and observation

$$
m^{2} \leq \frac{24}{5} F(e)\left(\frac{2 \pi \hbar}{c^{2} P_{\mathrm{b}}}\right)^{2} \frac{\dot{P}_{b_{o b s}}-\dot{P}_{b_{f(R)}}}{\dot{P}_{b_{f(R)}}}
$$

where

$$
F(e)=\frac{1+\frac{73}{24} e^{2}+\frac{37}{96} e^{4}}{\left(1-e^{2}\right)^{3}} .
$$

From the data on Table 3.2 we obtain the following upper limit

$$
m_{g}<5.95 \times 10^{-20} \mathrm{eV} / c^{2}
$$

comparable to the constraint derived from PSR $B 1913+16$. The constrain is similar to the one obtained by Antoniadis et al. (2013) in Brans-Dicke theory.

\subsection{Conclusions and future perspectives}

In this chapter we have computed the gravitational emission from binary systems in the post-Minkowskian limit of Extended Theories of Gravity. In this framework, GW have other polarizations modes than the plus $(+)$ and cross $(\times)$ modes predicted 
by General Relativity (Capozziello and De Laurentis, 2011). Extended Theories of Gravity allow massive and ghosts modes that could be detected using ground-based interferometric detectors, like VIRGO and LIGO (Abbott et al., 2009), and the future space-based interferometric detector, like eLISA (Amaro-Seoane et al., 2013). We have shown that ETGs can also be tested using timing array data from binary pulsars. We extended the quadrupole emission formalism of GR to analytic $f(R)$ models of gravity, computing model parameters. First, we derive an analytical expression of the quadrupole radiation rate singling out the GR and $f(R)$-gravity contributions. We have compared the predictions of both theories using a sample of relativistic binary systems. The main source of error in our estimates is the uncertainty in the mass of each component. Another source of uncertainty is the amount of mass transfer between the components (if any). If there is no matter accretion or mass loss within the system, the discrepancy between the GR prediction and the observations could be an indication of gravitational effects described by an analytical $f(R)$-theory of gravity.

Next, from the data on PSR $J 0348+0432$ we set an upper bound on the mass of the graviton. While the data is compatible with the GR prediction, it does not rule out ETGs. Our bound is comparable with other bounds derived from galaxies, clusters of galaxies and other sources (Antoniadis et al., 2013). To improve our results, we would need to compute the Post-Keplerian parameters and masses of the system using the analytic $f(R)$-theory itself and not just its Newtonian limit, without making specific assumptions about the mass of the pulsar or the inclination of the orbit. Second, we would need to take into account the hydrodynamical effects due to the transfer of the matter within the binary system. About the former, the best solution would be to obtain mass estimates using "theory-independent" methods such as spectroscopic techniques.

From our analysis of star formation and gravitational wave emission we have shown that $f(R)$-gravity can explain phenomena similarly to GR in the weak and strong field limits without invoking any chameleon mechanism. We made predictions that could potentially discriminate GR from ETGs. Our next step will be to test the analytic $f(R)$ models at more "proper" scales. Since the dynamics of galaxies have been extensively studied in the literature we will concentrate in the scale of cluster of galaxies and at cosmological scales. 


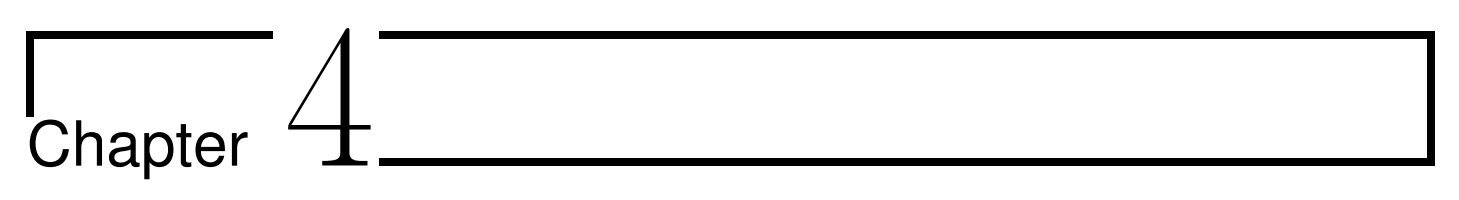

\section{Cluster pressure profiles in Extended Theory of gravity}

\section{Contents}

4.1 The Sunyaev-Zel'dovich effect: cluster of galaxies . . . . 63

4.1 .1 Cluster properties ............... . 64

4.1 .2 Isothermal $\beta$-profile . . . . . . . . . . . . 65

4.1.3 Komatsu-Seljak profile. . . . . . . . . . . . . . 66

4.1.4 The universal pressure profile . . . . . . . . . . 69

$4.2 f(R)$ pressure profile ............... 70

4.3 Data and methodology .............. 71

4.3.1 X-ray Cluster Catalog . . . . . . . . . . . . . 72

4.3.2 Cosmic Microwave Background data . . . . . . . . . . . . . 73

4.3.3 The predicted thermal Sunyaev-Zeldovich profile . . . . . . 75

4.4 Estimation of the $f(R)$ parameters . . . . . . . 77

4.5 Discussion and future perspectives .......... 79

T Tersions of Extended Theories of Gravity that modify the Newtonian potential in $V$ the weak field limit could explain the accelerated expansion of the Universe and, at the same time, describe the clustering of the large scale structure without requiring DM. Since, the exact functional form of the $f(R)$-Lagrangian is unknown, we need to combine theoretical considerations with observations to build a theoretical framework where to accommodate the astronomical observations. Thus, it is important to test potential models using all available data in order to predict the exact functional form of the $f(R)$-Lagrangian directly from the observations.

Analytic $f(R)$ models give rise to a Yukawa-like correction of the gravitational potential in the Post Newtonian limit (see for details Appendix B). These models have been used to explain the flat rotation curves of spiral galaxies (Cardone and Capozziello, 2011) and the velocity dispersion of elliptical galaxies (Napolitano et al., 
2012).

Clusters are the largest virialized object in the Universe and will allow us to test $f(R)$ on scales larger than galactic ones, intermediate between stellar and cosmological scales. Capozziello et al. (2009a) showed that ETGs are a viable alternative to the DM halo model. They provide a description of the distribution of baryonic matter (stars+gas) in agreement with observations. This study considered X-ray observations of 12 clusters. Schimdt et al. (2009) and Ferraro et al. (2011) have constrained particular models of $f(R)$ gravity using the number of cluster found in numerical simulations. Since $f(R)$ models have more degrees of freedom than GR, simulations are required to be specific for each particular Lagrangian so only one model can be constrained per simulation.

A more promising tool to constrain ETGs using clusters is based on the SZ effect (Sunyaev and Zeldovich $(1972,1980)$; a technical presentation of the effect will be given below). Clusters generate temperature fluctuation in the Cosmic Microwave Background. Several groups have recently reported measurement of the TSZ effect. Data was taken by the Atacama Cosmology Telescope (ACT) (Hand et al. (2011), Sehgal et al. (2011), Hasselfield et al. (2013), Menanteau et al. (2013)), the South Pole Telescope (SPT) (Staniszewski et al. (2009), Vanderlinde et al. (2010), Williamson et al. (2011), Benson et al. (2013)) and the Planck satellite (Planck Collaboration (2012, 2013l,p,r)). The data present several discrepancies with the theoretical expectations based on the $\Lambda$ CDM model. WMAP 7 yrs data (Komatsu et al., 2011) have shown that the contribution of the unresolved cluster population is smaller than expected. Planck Collaboration $(2012,2013 \mathrm{r}$ ) found the amplitude of the TSZ effect in the Coma cluster was $\sim 10-15 \%$ lower than the value expected based on X-ray observations. These discrepancies can be explained in two different ways: (A) they are due to the existence of complex structures and substructures in clusters of galaxies, (B) they reflect a limitation of the theoretical modeling (Fusco-Femiano et al., 2013). To study if these discrepancies are due to the theoretical model, we have constructed pressure profiles of cluster of galaxies in ETGs. We assume that the gas is in hydrostatic equilibrium within the potential well of the modified gravitational field of the cluster, but we do not assume the existence of any DM component. The assumption of the hydrostatic equilibrium has been verified to be valid for the intermediate regions of clusters using numerical simulations based on the concordance cosmology. In the inner regions, the physics of baryons is more complex, while in the outer regions it is dominated by non-equilibrium processes (Kravtsov and Borgani, 2012, Shi and Komatsu, 2014).

Since it is crucial to test those models on different scales, in this chapter we will construct the pressure profiles of 579 cluster of galaxies and we will compare them with those measured using the Thermal Sunyaev-Zeldovich (TSZ) effect on the Planck 
foreground clean map SMICA.

\subsection{The Sunyaev-Zel'dovich effect: cluster of galaxies}

When CMB photons cross a cluster of galaxies, they are scattered by the free electrons residing in its deep potential well. In the 1970's R. Sunyaev and Ya. Zel'dovich described the temperature anisotropies imprinted on the CMB radiation due to this interaction. Observations of these anisotropies are currently carried out and have become a powerful observational probe to study the evolution of structure in the Universe.

Clusters are the largest virialized objects in the Universe, with a virial mass in the range $10^{13} \div(\mathrm{few}) 10^{15} \mathrm{M}_{\odot}$. The mass of baryons in cluster is composed at least by two components, A) diffuse Intra Cluster Medium (ICM), and B) stars. The baryon fraction in cluster is

$$
\frac{M_{g a s}}{M_{c l}}+\frac{M_{s t a r}}{M_{c l}} \simeq 0.07 h^{-1.5}+0.05
$$

therefore, most of baryons in clusters are not in, galaxies but are in the diffuse ICM (White et al. (1993), White and Fabian (1995), Lubin et al. (1996)). The gas is highly ionized, with temperatures larger than $T_{e}>1 \mathrm{keV}$. The incoming CMB photons are inverse Compton scattered by the free electrons and gain energy when crossing the potential wells of clusters. As a result of the interaction, the CMB blackbody spectrum is distorted and clusters induce secondary temperature anisotropies on the CMB. We can distinguish two SZ components: the thermal component (hereafter TSZ, Sunyaev and Zeldovich (1972)) due to the thermal motion of the electrons in the potential well of the cluster and the kinematic (KSZ, Sunyaev and Zeldovich (1980)) component due to the motion of the cluster as a whole with respect to the rest frame defined by the Cosmic Microwave Background. Neglecting relativistic corrections, the TSZ and KSZ contributions in the direction of a cluster $\hat{n}$ are given by

$$
\frac{T(\hat{n})-T_{0}}{T_{0}}=\int\left[g(\nu) \frac{k_{B} T_{e}}{m_{e} c^{2}}+\frac{\vec{v}_{c l} \hat{n}}{c}\right] d \tau
$$

where, $k_{B}$ the Boltzmann constant, $m_{e} c^{2}$ the electron annihilation temperature, $c$ the speed of light and $\nu$ the frequency of observation and $\vec{v}_{c l}$ the peculiar velocity of the cluster. We denoted $d \tau=\sigma_{T} n_{e} d l$ the cluster optical depth to the SZ effect, with $\sigma_{T}$ Thomson cross section, $n_{e}(l)$ the electron density evaluated along the line of sight $l ; T_{0}$ is the current Cosmic Microwave Background blackbody temperature and $g(\nu)$ is the frequency dependence of TSZ effect. It is customary to introduce the Comptonization parameter defined as

$$
y_{c}=\frac{k_{B} \sigma_{T}}{m_{e} c^{2}} \int n_{e}(r) T_{e}(r) d l=\frac{\sigma_{T}}{m_{e} c^{2}} \int P_{e}(r) d l
$$

In the non-relativistic limit,

$$
g(x)=x \operatorname{coth}(x / 2)-4
$$


where the reduced frequency $x$ is given by $x=h \nu / k_{B} T$.

Several properties make the SZ effect a very important tool in Cosmology. First, the distortion once it is produced does not decay with distance, making it a very useful tool to detect clusters at high redshifts. Second, the TSZ effect depends on frequency. No known astrophysical sources follow the same law so clusters can be distinguish from other foreground contributions. For very hot clusters $\left(T_{e}>10 \mathrm{keV}\right)$, relativistic corrections must be included (Itoh et al., 1998, Nozawa et al., 1998). The extra terms due to the relativistic corrections are given in Appendix E. As an illustration, in Fig. 4.1 we show the effect of the relativistic correction for a cluster with electron temperature in the range $T_{e}=[2,10] \mathrm{keV}$. The frequency dependence of the TSZ distortion is independent of redshift. The figure shows $g(\nu)$ without (solid black line, see eq. (4.4)) and with relativistic corrections at different temperatures (color lines).

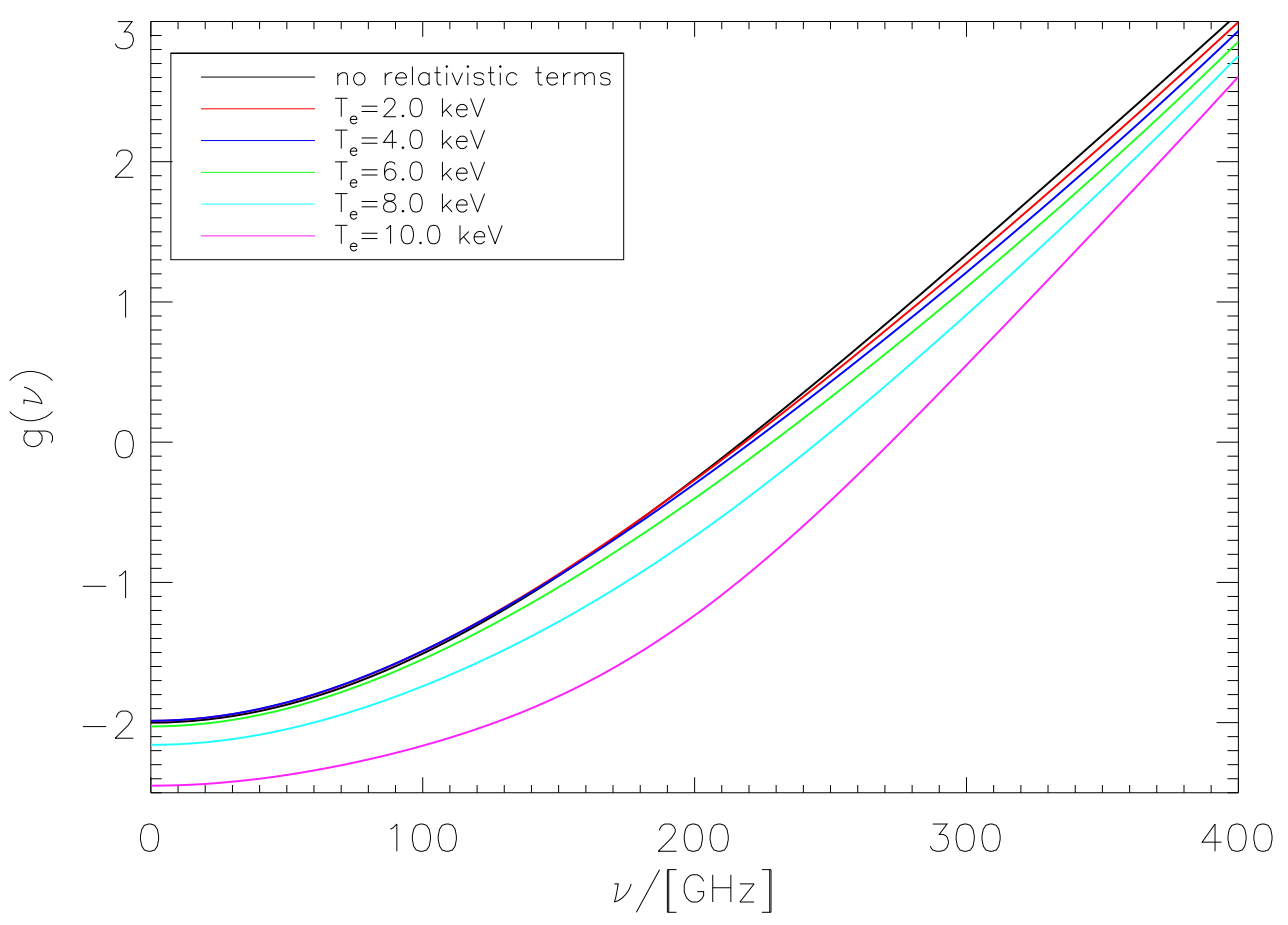

Figure 4.1: Spectral dependence of SZ effect in the cluster. Spectral dependence of the TSZ effect without relativistic corrections (black line) and including the relativistic at different temperatures.

\subsubsection{Cluster properties}

In clusters, dynamical estimates of the mass indicate that DM constitutes about $85 \%$ of the total mass. Typically they contain from hundreds to up to one thousand galaxies within a region of $2 \mathrm{Mpc}$, but their mass represents a mere $3 \%$ of the total mass of the cluster. Most of the baryon mass resides in a hot Inter Cluster Medium 
gas, making up to $12 \%$ of the total mass, with temperatures in the range from $10^{7}$ to $10^{8} \mathrm{~K}$. The ICM is highly rarefied; electron number densities are typically $n_{e} \sim 10^{-4}-$ $10^{-2} \mathrm{~cm}^{-3}$. Clusters are strong X-rays sources, with a luminosity $L_{X} \sim 10^{43}-10^{45} \mathrm{erg} / \mathrm{s}$; they represent the largest virialized systems in the Universe. The virial radius is typically $r_{v i r} \sim 1-3 \mathrm{Mpc}$. We can define the virial mass $M_{v i r}$ of the cluster as the mass enclosed within this radius. The virial mass ranges from $\sim 10^{13} \mathrm{M}_{\odot}$ for rich groups of galaxies to $\sim 10^{15} \mathrm{M}_{\odot}$ for the more massive systems. It is given by

$$
M_{v i r}=\frac{4 \pi}{3}\left[\Delta_{c}(z) \rho_{c}(z)\right] r_{v i r}^{3},
$$

where $\Delta_{c}(z)$ is the overdensity of a collapsed spherical and uniform mass distribution (top hat). It depends on the cosmological parameters of the background model, $\Omega_{m}$ and $\Omega_{\Lambda}$ (Bryan and Norman, 1998).

Other definitions of mass in terms of different length scales also appear often in the literature. The radius $r_{500}$, defined as the radius at which the mean overdensity of the cluster is 500 times the critical density of the Universe at the same redshift $\rho_{c}(z)=2.775 \times 10^{11} E^{2}(z) h^{2} M_{\odot} \mathrm{Mpc}^{-3}$. This definition is rather convenient since scaling relations based on numerical simulations and X-ray observations exist that allow to determine $r_{500}$ for individual clusters. For instance (Böhringer et al., 2007)

$$
r_{500}=\frac{(0.753 \pm 0.063) h^{-1} \mathrm{Mpc}}{h(z)}\left(\frac{L_{X}}{10^{44} h^{-2} \mathrm{erg} \mathrm{s}^{-1}}\right)^{0.228 \pm 0.015} .
$$

By definition, $M_{500}$ is the mass enclosed within $r_{500}$ :

$$
M_{500} \equiv \frac{4 \pi}{3}\left[500 \rho_{c}(z)\right] r_{500}^{3} .
$$

In Table 4.1 we summarize typical magnitudes of clusters collected from the literature.

\subsubsection{Isothermal $\beta$-profile}

At temperatures in the range $T_{e}=0.5 \div 15 \mathrm{keV}$, the ICM emits at X-ray frequencies due to thermal Bremsstrahlung. This emission can be used to characterize the mass and radial density profile of the gas. One of the first to propose a radial distribution for the ICM were Cavaliere and Fusco-Femiano $(1976,1978)$. They suggested that if gas and galaxies are in equilibrium in the potential $\phi(r)$ of the cluster, their radial distribution, represented by $\rho_{\text {gal }}$ and $n_{\text {gas }}$ are related through the following equation

$$
\frac{1}{n_{\text {gas }}(r)} \frac{d}{d r}\left[\frac{k_{B} T_{\text {gas }}(r)}{\mu m_{p}} n_{\text {gas }}(r)\right]=\frac{1}{\rho_{\text {gal }}(r)} \frac{d\left(\sigma_{r} \rho_{\text {gal }}(r)\right.}{d r}
$$

where $m_{p}$ the proton mass, $\mu$ the mean molecular weight, $T_{g a s}(r)$ the gas temperature and $\sigma_{r}$ the galaxy velocity dispersion, assumed to be isotropic. For an isothermal case, 


\begin{tabular}{|cccccccc|}
\hline \hline $\begin{array}{c}l \\
(\mathrm{deg})\end{array}$ & $\begin{array}{c}b \\
(\mathrm{deg})\end{array}$ & $z$ & $\begin{array}{c}T_{e}^{X} \\
(\mathrm{keV})\end{array}$ & $\begin{array}{c}L_{X} \\
\left(10^{44} \mathrm{erg} / \mathrm{s}\right)\end{array}$ & $\begin{array}{c}n_{e 0} \\
\left(\mathrm{~m}^{-3}\right)\end{array}$ & $\begin{array}{c}r_{500} \\
(\mathrm{Mpc})\end{array}$ & $\begin{array}{c}M_{500} \\
\left(10^{15} M_{\odot}\right)\end{array}$ \\
\hline \hline 107.2 & -45.2 & 0.12 & 1.48 & 3.84 & 1990 & 0.77 & 0.26 \\
177.9 & -53.5 & 0.19 & 4.38 & 5.89 & 3550 & 0.95 & 0.50 \\
220.6 & -38.5 & 0.07 & 0.63 & 2.77 & 2770 & 0.64 & 0.15 \\
263.7 & -22.5 & 0.16 & 6.11 & 6.74 & 3770 & 1.03 & 0.65 \\
152.7 & 33.8 & 0.12 & 1.17 & 3.51 & 2980 & 0.72 & 0.22 \\
259.4 & 40.2 & 0.08 & 0.60 & 2.73 & 4210 & 0.63 & 0.15 \\
66.7 & 68.5 & 0.16 & 3.12 & 5.14 & 5810 & 0.89 & 0.41 \\
11.4 & 49.4 & 0.04 & 1.39 & 3.74 & 9340 & 0.78 & 0.27 \\
28.9 & 44.5 & 0.03 & 1.60 & 3.95 & 1790 & 0.81 & 0.30 \\
50.4 & 31.2 & 0.16 & 3.74 & 5.53 & 3520 & 0.93 & 0.46 \\
54.0 & -45.1 & 0.06 & 1.28 & 3.63 & 4740 & 0.76 & 0.25 \\
348.3 & -64.8 & 0.06 & 2.25 & 4.52 & 15360 & 0.86 & 0.38 \\
38.9 & -69.3 & 0.10 & 1.00 & 3.30 & 5520 & 0.71 & 0.21 \\
81.3 & -68.5 & 0.08 & 1.28 & 3.62 & 4000 & 0.75 & 0.25 \\
57.0 & 88.0 & 0.02 & 3.53 & 5.41 & 3860 & 0.97 & 0.53 \\
\hline \hline
\end{tabular}

Table 4.1: Magnitudes (observed and derived) of clusters obtained from the literature Kocevski and Ebeling (2006): $(l, b)$ are the galactic latitude and longitude, $z$ the redshift, $L_{X}, T_{X}$ the $\mathrm{X}$-ray luminosity and central electron temperature, $n_{e 0}$ the central electron density, and $r_{500}$, $M_{500}$ the derived radii derived masses.

$T_{\text {gas }}$ and $\sigma_{r}$ are independent of position and eq. (4.8) leads to an electron number density that follows the so-called isothermal $\beta$-model

$$
n_{e}(r)=n_{e 0}\left(1+\left(\frac{r}{r_{c}}\right)^{2}\right)^{-3 \beta / 2}
$$

where $n_{e 0}$ is the central density and $r_{c}$ a scale length characteristic of every cluster and known as core radius. The observed values of $\beta$, obtained from X-ray surface brightness profiles is found to be in the range $\beta=[0.6-0.8]$ (Jones and Forman, 1984). To estimate SZ effect it is necessary to integrate the radial density and temperature profiles to obtain the Comptonization parameter as shown by the eq. (4.3).

The $\beta$-profile has been used extensively in X-ray studies of the clusters (for more details see Sarazin (1986), Birkinshaw (1999)). However, it only represents a good fit for the inner part of clusters and overpredicts the pressure profile outside $r_{c}$ (AtrioBarandela et al., 2008).

\subsubsection{Komatsu-Seljak profile.}

The $\beta$ model is a good fit to the X-ray emitting region of clusters but fails to describe the cluster outskirts so other profiles have been introduced. Komatsu and Seljak (2001) (KS) constructed a cluster pressure profile assuming the gas is in hydrostatic equilibrium within the gravitational potential generated by the dark matter, described 
by a Navarro-Frenk-White (NFW) profile (Navarro et al., 1997). In addition, the gas is assumed to follow a polytropic equation of state, $P \propto \rho^{\gamma}$. Then, on top of the parameters needed to describe the dark matter distribution, we need two parameters to describe the gas: the polytropic index $\gamma$ and the central electron pressure $P(0)$. In the KS model, one further imposes that gas and dark matter follow the same profile at some fix radius (around the virial radius) it is possible to fix the polytropic index, leaving only the central pressure as a free parameter. The KS profile must be considered as an average model since it does not take into account effects such non-thermal pressure, gas cooling or star formation that could alter the profile of individual clusters (Bode et al., 2009, Shi and Komatsu, 2014).

To write down the KS profile, first we rewrite the gas pressure profile as

$$
P_{\text {gas }}(r)=P_{\text {gas }}(0)\left[y_{\text {gas }}\left(r / r_{s}\right)\right]^{\gamma},
$$

where $r_{s} \equiv \frac{r_{v i r}}{c}$ represents the scale radius of the NFW DM profile, with $c$ a concentration parameter and $r_{v i r}$ the virial radius. The equation of hydrostatic equilibrium and Poisson equation give the following solution for the gas pressure profile

$$
y_{\text {gas }}(x) \equiv\left\{1-B\left[1-\frac{\ln (1+x)}{x}\right]\right\}^{1 /(\gamma-1)},
$$

where the quantities

$$
\begin{gathered}
B \equiv 3 \eta^{-1}(0) \frac{\gamma-1}{\gamma}\left[\frac{\ln (1+c)}{c}-\frac{1}{1+c}\right]^{-1}, \\
\gamma=1.137+8.94 \times 10^{-2} \ln (c / 5)-3.68 \times 10^{-3}(c-5),
\end{gathered}
$$

and

$$
\eta(0)=2.235+0.202(c-5)-1.16 \times 10^{-3}(c-5)^{2} .
$$

are obtained by numerical fits to the solutions. The central pressure, $P_{\text {gas }}(0)$, is given by

$$
P_{g a s}(0)=55.0 h^{2} \mathrm{eV} \mathrm{cm}^{-3}\left[\frac{\rho_{\text {gas }}(0)}{10^{14} h^{2} M_{\odot} \mathrm{Mpc}^{-3}}\right]\left[\frac{k_{B} T_{g a s}(0)}{8 \mathrm{keV}}\right],
$$

where $T_{\text {gas }}(0)$ is the central gas temperature given by the following relation

$$
k_{B} T_{\text {gas }}(0)=8.80 \mathrm{keV} \eta(0)\left[\frac{M_{v i r} /\left(10^{15} h^{-1} M_{\odot}\right)}{r_{v i r} /\left(1 h^{-1} \mathrm{Mpc}\right)}\right] .
$$

In order to determine the central gas density $\rho_{\text {gas }}(0)$, one needs to assume that its value is equal to $\rho_{\text {gas }}\left(r_{v i r}\right)=\frac{\Omega_{b}}{\Omega_{m}} \rho_{d m}\left(r_{v i r}\right)$, where $\Omega_{b} / \Omega_{m}$ is the cosmic baryon fraction. Using this assumption we obtain

$$
\begin{aligned}
\rho_{\text {gas }}(0)= & 7.96 \times 10^{13} h^{2} M_{\odot} \operatorname{Mpc}^{-3}\left(\frac{\Omega_{b}}{\Omega_{m}}\right) \frac{M_{\text {vir }} /\left(10^{15} h^{-1} M_{\odot}\right)}{\left[r_{\text {vir }} /\left(1 h^{-1} \mathrm{Mpc}\right)\right]^{3}} \times \\
& \times \frac{c^{2}}{(1+c)^{2}} \frac{1}{y_{\text {gas }}(c)}\left[\ln (1+c)-\frac{1}{1+c}\right]^{-1} .
\end{aligned}
$$


Nevertheless, since $\frac{\Omega_{b}}{\Omega_{m}}$ is just an upper limit of the baryon fraction in clusters one can expect that the observed gas pressure to be smaller.

For any given clusters, the relation between the viral mass and the mass within $r_{500}$ requires to integrate the matter distribution with radius. For the NFW profile (Navarro et al., 1997)

$$
\rho_{\mathrm{NFW}}(r)=\frac{\rho_{\mathrm{s}}}{\left(r / r_{\mathrm{s}}\right)\left(1+r / r_{\mathrm{s}}\right)^{2}},
$$

one ends up solving the following non-linear equation for $M_{v i r}$ :

$$
M_{v i r} \frac{m\left(c r_{500} / r_{v i r}\right)}{m(c)}=M_{500}
$$

where $m(x) \equiv \ln (1+x)-x /(1+x)$, and $r_{v i r}$ is related to $M_{v i r}$ by eq (4.5).

In Fig. 4.2 we represent the ratios $r_{500} / r_{v i r}$ and masses $M_{500} / M_{v i r}$ as function of the concentration parameter $c$. Both ratios were computed at $z=0$ for the $\Lambda$ CDM model with $\Omega_{m} \sim 0.27$ and $\Delta_{c}(0) \simeq 95$.

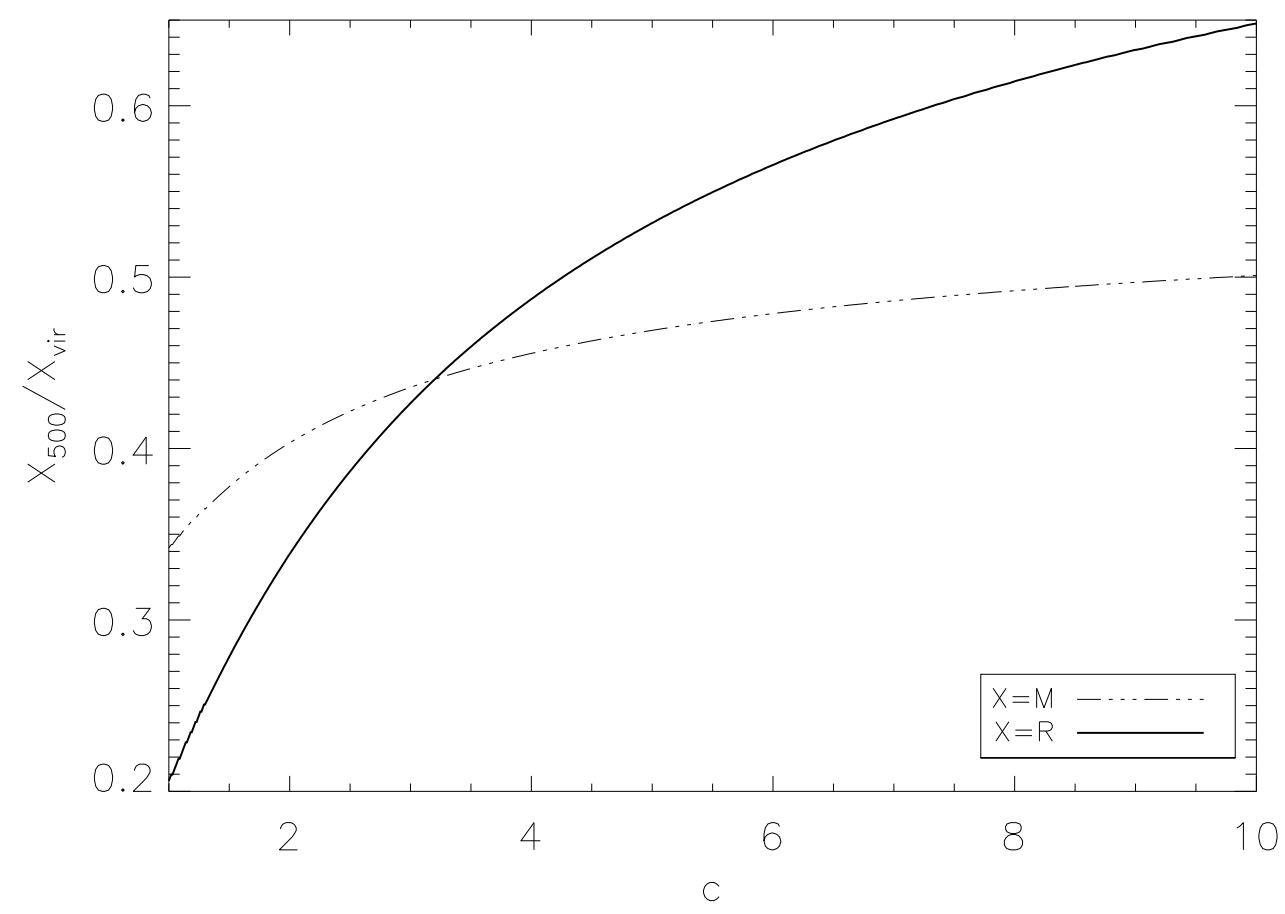

Figure 4.2: Ratios $r_{500} / r_{v i r}$ (solid line) and overdensity mass to virial mass $M_{v i r} / M_{500}$ (dotdashed line) as a function of the concentration parameter, in the concordance $\Lambda \mathrm{CDM}$ model at $z=0$.

Finally, to construct the profile one still needs to specify the concentration parameter. Seljak (2000) showed that

$$
c_{\text {seljak }}=\frac{5.09}{1+z}\left(\frac{M_{\mathrm{vir}}}{10^{14} h^{-1} M_{\odot}}\right)^{-0.2},
$$


More recent $N$-body simulations gave a slightly different scaling (Duffy et al., 2008)

$$
c_{d u f f y}=\frac{5.72}{(1+z)^{0.71}}\left(\frac{M_{\mathrm{vir}}}{10^{14} h^{-1} M_{\odot}}\right)^{-0.081}
$$

This formula makes clusters of galaxies $\left(M_{\odot} \gtrsim 10^{14} M_{\odot}\right)$ more concentrated than $c_{\text {seljak }}$ would predict.

\subsubsection{The universal pressure profile}

The interest for obtaining a good description of the temperature anisotropies on the CMB generated by clusters led to propose phenomenological parameterizations based on the generalized Navarro-Frank-White profiles (Arnaud et al., 2010). The proposed profile is

$$
P_{e}(x)=1.65(h / 0.7)^{2} \mathrm{eV} \mathrm{cm}^{-3} E^{8 / 3}(z) \times\left[\frac{M_{500}}{3 \times 10^{14}(0.7 / h) M_{\odot}}\right]^{2 / 3+\alpha_{p}} p(x),
$$

where $x=r / r_{500}, \alpha_{p}=0.12, E(z)=\left[\Omega_{m}(1+z)^{3}+\Omega_{\Lambda}\right]^{1 / 2}$ for $\Lambda \mathrm{CDM}$ and

$$
p(x) \equiv \frac{P_{0}}{\left(c_{500} x\right)^{\gamma}\left[1+\left(c_{500} x\right)^{\alpha}\right]^{(\beta-\gamma) / \alpha}},
$$

The parameters were derived using X-Ray data of 33 clusters with masses in the range $10^{14} \leq M_{500} \leq 10^{15}$ and redshift $z \leq 0.2$ and the results of numerical simulations to fit the outer parts of clusters.

Recently, the Planck Collaboration has fit eq. (4.22) profiles to the combined SZ and X-Ray. They have reconstructed the gas mass fraction profile out to $3 r_{500}$ for a sample of 62 nearby massive clusters from the Planck cluster Catalog within the mass range $0.2 \leq M_{500}\left(10^{15} M_{\odot}\right) \leq 2$ with redshifts $z \leq 0.5$ (Planck Collaboration (2011a,b, 2013o,q)). Similarly, Sayers et al. (2013) analyzed 45 massive clusters with median mass $M_{500}=9 \times 10^{14} M_{\odot}$ and median redshift $z=0.42$. All the best-fit values regarding the cluster universal pressure profile are summarized in Table 4.2.

\begin{tabular}{|cccccc|}
\hline \hline Profile Model & $c_{500}$ & $\alpha$ & $\beta$ & $\gamma$ & $P_{0}$ \\
\hline \hline Arnaud 2010 & 1.177 & 1.051 & 5.4905 & 0.3081 & $8.403 h_{70}^{3 / 2}$ \\
Planck 2012 & 1.81 & 1.33 & 4.13 & 0.31 & 6.41 \\
Sayers 2013 & 1.18 & 0.86 & 3.67 & 0.67 & 4.29 \\
\hline \hline
\end{tabular}

Table 4.2: Best-fit parameter of the universal pressure profile from X-ray and SZ observations.

With the upcoming of Planck, South Pole and Atacama Cosmology Telescope data, the TSZ effect of more than 300 clusters has been measured. This opens the possibility of using cluster pressure profiles to test gravity on Mpc scales. 


\section{$4.2 \quad f(R)$ pressure profile}

The dynamical effect of DM in galaxy clusters can be replaced by a Yukawa-like correction to the Newtonian potential in analytical $f(R)$ theories, eq. (1.82). Eq. (1.82) describes the gravitational potential in $f(R)$ gravity. We will assume that this is the potential of clusters of galaxies. To construct their pressure profile we will assume that the gas is in hydrostatic equilibrium within the modified potential well

$$
\frac{d P(r)}{d r}=-\rho(r) \frac{d \Phi(r)}{d r},
$$

and the gas follows a polytropic equation of state

$$
P(r) \propto \rho^{\gamma}(r)
$$

The system of equations (1.82),(4.24) and (4.25) together with the equation for the mass conservation

$$
\frac{d M(r)}{d r}=4 \pi \rho(r)
$$

form a closed system that can be solved numerically to compute the pressure profiles of any cluster as a function of the two extra gravitational parameters $(\delta, L)$ and the polytropic index $\gamma$. We integrate eqs. (1.82),(4.24), (4.25), (4.26) on a grid centered on the cluster, as shown in Fig. 4.3a; the blue square represents the cluster center. Once the pressure has been computed at each point in the grid, it is integrated along the line of sight, represented by the red line. The resulting pressure profile as a function of angular separation is shown in Fig. 4.3b. The red square corresponds to the line of sight of Fig. 4.3a. The pressure profile of Fig. 4.3b does not include a Dark

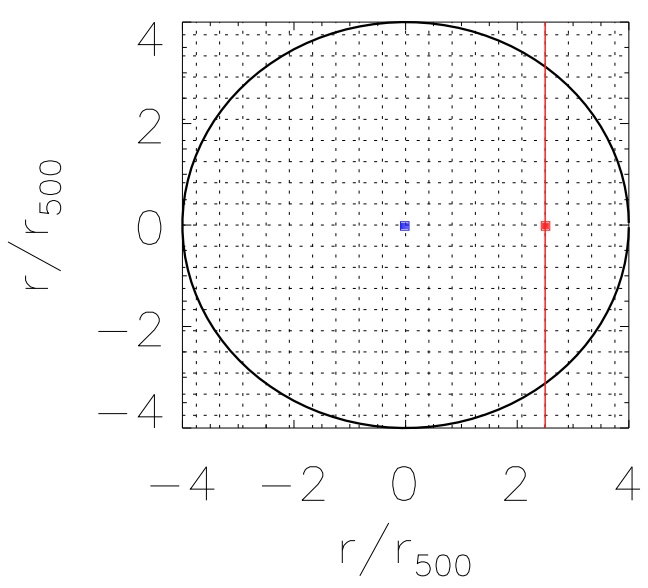

(a)

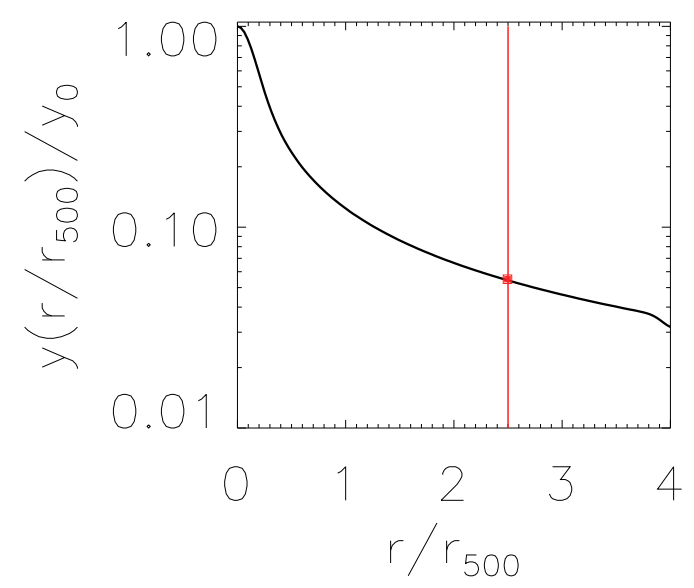

(b)

Figure 4.3: (a) Grid use for the numerical solution of eqs. (1.82),(4.24), (4.25) and (4.26). The blue square represents the center of the cluster and the red line one line of sight. (b) resulting pressure profile. The red square corresponds to the line of sight of (a).

Matter component, only baryons in hydrostatic equilibrium within a Yukawa modified 
Newtonian potential. Since there is no DM in our model, the density $\rho(r)$ is meant to represent $\rho_{\text {gas }}(r)$. In Fig. 4.4, we compare the pressure profile of the Coma cluster, integrated along the line of sight, of analytic $f(R)$ with the DM models with NFW profiles given in Table 4.2. For convenience, all distances are written in units of $r_{500}$ and the angular scale is $\theta_{500}=r_{500} / d_{A}^{C o m a}$ where $d_{A}^{C o m a}$ is the angular diameter distance of Coma. Coma cluster is the last one in Table 4.1, Dashed, solid and dash-dotted lines correspond to universal profiles with the Arnaud et al. (2010), Planck Collaboration (2012) and Sayers et al. (2013) parameters, respectively. The long-dashed line corresponds to the Komatsu-Seljak model (rewritten, for sake of convenience, in terms of $\left.r_{5} 00\right)$ with concentration parameter $c=5.16$ and scale radius $r_{s}=0.37 \mathrm{Mpc}$, the green solid line to the $\beta$ model (see parameter in last line of Table 4.1), and the red solid line to the $f(R)$ model with $\delta=-0.98$ and $L=1 \mathrm{Mpc}$.

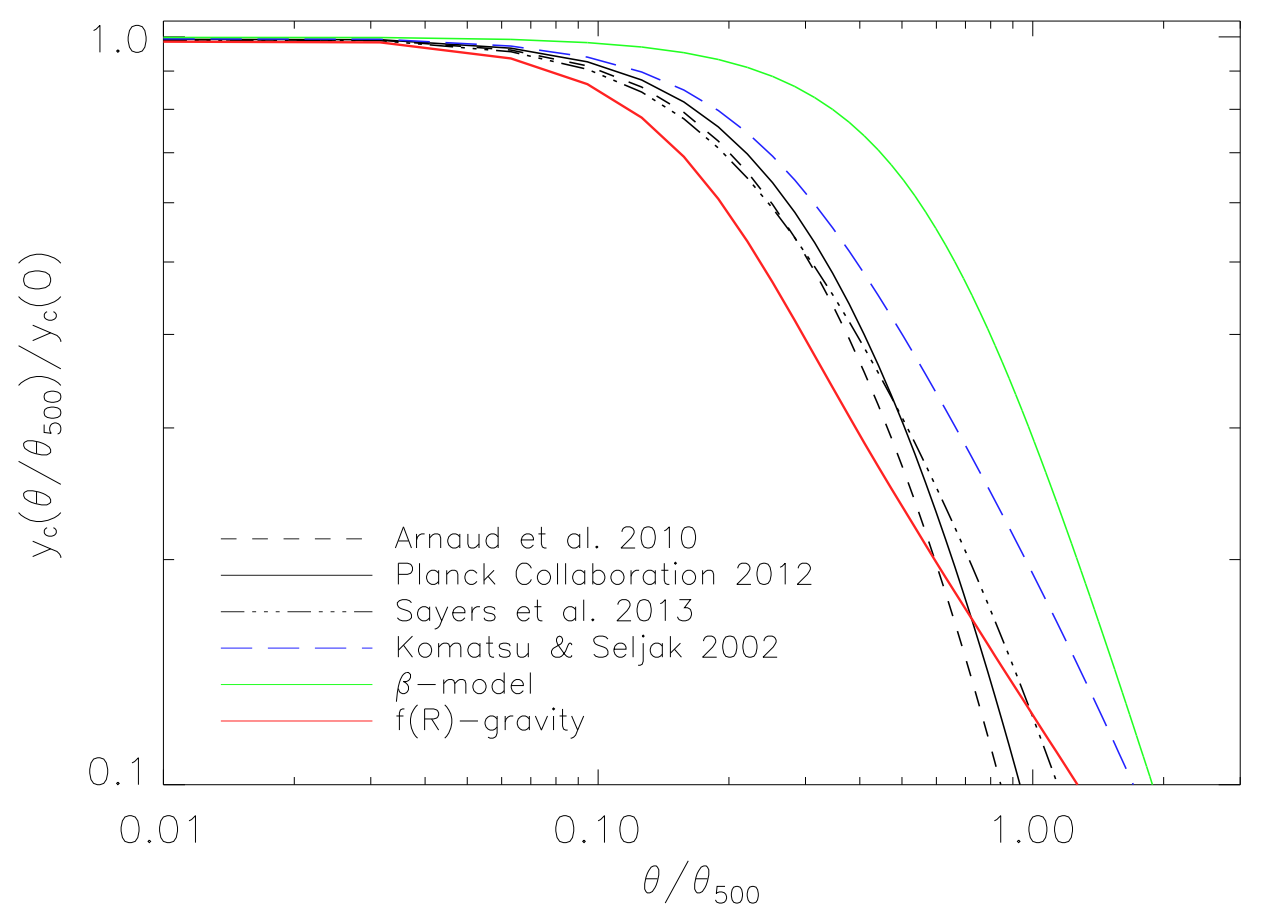

Figure 4.4: Pressure profiles integrated along the line of sight for the Coma cluster. The $f(R)$ model (red solid line), three universal profiles (dashed, solid and dash-dotted lines), the Komatsu-Seljak (long dashed line) and $\beta=2 / 3$ models are shown. Parameters of the universal profiles are given in Table 4.2. The angular diameter distance is that of the Coma cluster $(z=0.023)$.

\subsection{Data and methodology}

To constrain ETGs using clusters of galaxies we will compare the cluster profiles computed in the previous section for a sample of 579 clusters with Planck foreground 
clean SMICA data from April 13th, 2013 data release.

\subsubsection{X-ray Cluster Catalog}

Our X-ray Cluster Catalog was constructed from three flux limited cluster samples: the ROSAT-ESO Flux Limited X-ray catalog (REFLEX, see Böhringer et al. (2004)), the extended Brightest Cluster Sample (eBCS, see Ebeling et al. (1998, 2000)) and the Clusters in the Zone of Avoidance (CIZA, see Ebeling et al. (2002), and Kocevski et al. (2007)). The details of catalog are given in Kocevski and Ebeling (2006). Briefly, all three surveys have X-ray fluxes greater than $3 \times 10^{-12} \mathrm{erg} \mathrm{cm}^{-2} \mathrm{~s}^{-1}$ in the $[0.1$ 2.4] keV ROSAT band. REFLEX is composed of 447 clusters at redshift $z \leq 0.3$. Their declinations are $\delta<2.5^{\circ}$, and their Galactic latitudes are $|b|>20^{\circ}$. The eBCS catalog is centered on the Northern hemisphere $\left(\delta>0^{\circ}\right)$ and contains 290 clusters at Galactic latitude $|b|>20^{\circ}$, and at redshifts of $z \leq 0.3$. Finally, CIZA contains another 165 clusters at redshift below 0.3 .

In order to have an homogeneous sample of clusters, the physical properties were consistently computed using publicly available RASS data. To determine cluster positions, point sources within the aperture of $1.5 h_{50}^{-1} \mathrm{Mpc}$ radius were removed from the centroid of each cluster. The unabsorbed X-ray fluxes were obtained by measuring total X-ray count rates, and taking into account exposure time and background. From the fluxes, X-ray luminosities were determined using cosmological luminosity distance and a temperature-dependent $K$-correction. In the last step all clusters dominated by a point sources are removed and a flux cut at $3 \times 10^{-12} \mathrm{erg} \mathrm{cm}^{-2} \mathrm{~s}^{-1}$ is applied. At the end of processing, the catalog contains 349 REFLEX, 268 eBCS, and 165 CIZA clusters at $z \leq 0.3$, corresponding to 782 clusters over the entire sky. In our analysis we used the 579 clusters that were outside the minimal Planck mask (that removes $\sim 20 \%$ of the sky in the Plane of the Galaxy). Figure 4.5 shows distribution on the sky of the clusters used in this analysis.

For each cluster our catalog contains the X-ray electron temperature derived from the $L_{X}-T_{X}$ relation given in White and Forman (1997), the core radii $r_{c}$ and central electron densities $n_{e, 0}$ derived from ROSAT data. The spatial properties of the X-ray emitting gas were derived by fitting the $\beta$-model to the canonical value of $\beta=2 / 3$ (Jones and Forman, 1984).

Atrio-Barandela et al. (2008) have shown that the predicted values of the averaged cluster pressure profile and the measured one in WMAP 3yr data were in agreement with the $\beta$ model for the inner part of the clusters where the discrepancy between the TSZ prediction and observation was below 10\%. But they disagree in the outskirt, where the $\beta$ does not describe the cluster profile. Nevertheless, the data derived from $\mathrm{X}$-ray properties will be used to construct cluster pressure profiles. 


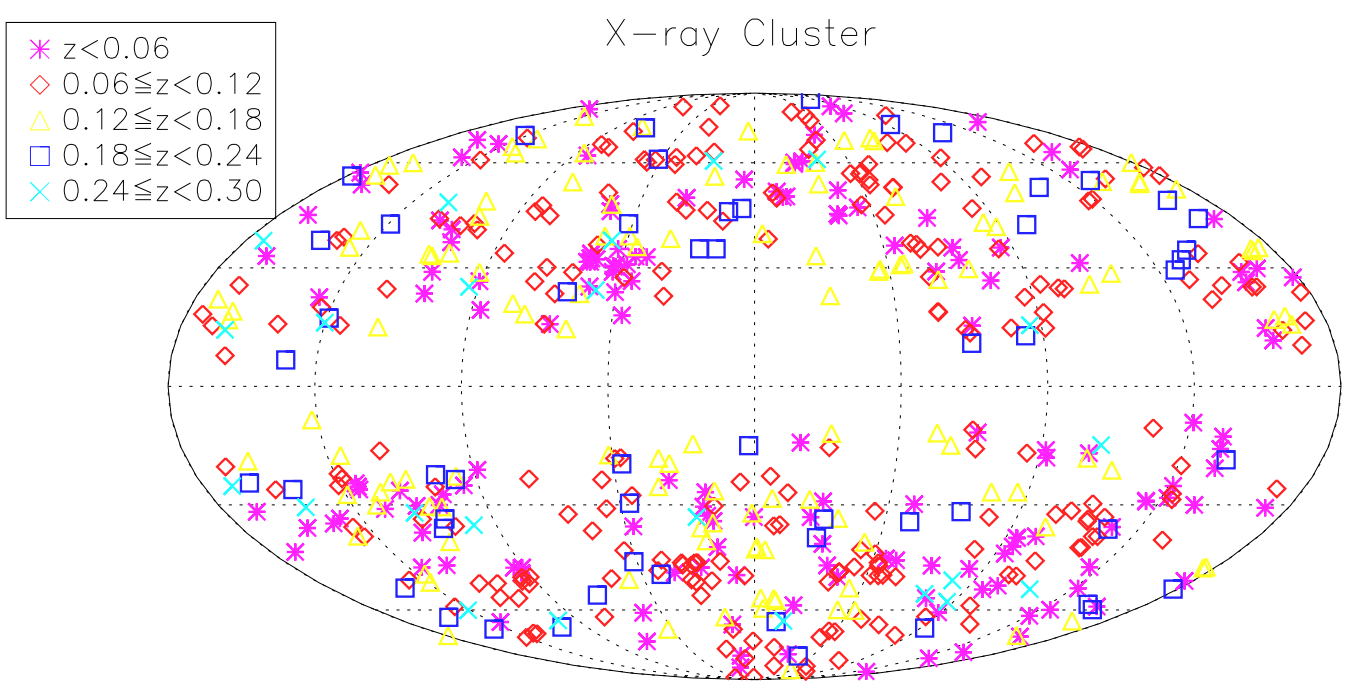

Figure 4.5: Distribution on the sky of the clusters used in this section.

\subsubsection{Cosmic Microwave Background data}

In 2013, the final WMAP 9 yrs data release was updated (Bennett et al., 2013) and Planck made public its first all sky data on CMB temperature anisotropies. Planck produced nine maps in the frequency range from 32 to $845 \mathrm{GHz}^{1}$. The high resolution, frequency coverage and low noise of Planck data allowed to determine the CMB power spectrum, set limits on primordial non-Gaussianity, measured the integrated Sachs-Wolfe (ISW) and gravitational lensing power spectra, etc (Planck Collaboration, 2013f,g,i,j,k,h,l,m,n,o). Unfortunately, the Planck Collaboration did not published foreground clean maps at all frequency channels as WMAP did. Instead, they used component separation methods to construct single Cosmic Microwave Background foreground clean maps combining all nine frequency channels (Planck Collaboration, 2013f) that would be the most useful data set for our study. Since foregrounds would dilute the cluster signal, we will use foreground clean data but without frequency information.

Component separation techniques reconstruct a map of CMB temperature fluctuations as an Internal Linear Combination (ILC) of all nine Planck frequencies. The SMICA map was constructed by given different weights to different frequencies in $\ell$ space. The Planck collaboration fit the amplitude and spectral parameters of CMB and foregrounds in the harmonic domain to remove the foreground contamination (Planck Collaboration, 2013f). All input maps have the same resolution and effective beam. This method products a map of 5 arcmin resolution. Other foreground clean maps have been constructed using other techniques. For comparison, we will replicate our analysis in the Needlet-Internal Linear Combination (NILC) map. NILC was constructed in Needlet space using ILC technique from 44 to $857 \mathrm{GHz}$, including mul-

\footnotetext{
${ }^{1}$ http://irsa.ipac.caltech.edu/Missions / planck.html
} 
tipoles up to $\ell=3200$. In this method, weights are different in different parts of the sky as well as on different multipoles. The mask used excludes the Galactic plane, the point sources and very bright regions of the sky. As for SMICA, all input maps have the same angular resolution Planck Collaboration (2013f).

Since these two maps were constructed using different techniques, they will differ in amplitude, distribution and spatial properties of the foreground residuals (Planck Collaboration, 2013g) so, by performing the analysis on both maps one can test for systematics. In Fig. 4.6 we plot the SMICA (a), NILC (b), mask (c) and cluster template used $(\mathrm{d})$. The template contains the pressure profiles of all clusters in our catalog in a $f(R)$ model with parameters $\delta=-0.99, L=0.5 \mathrm{Mpc}$ and polytropic index $\gamma=1.0$. For a better view, the scale is plot using a logarithmic scale. The resolution of our maps is Healpix $N_{\text {side }}=2048$ (Górski et al., 2005).

(a) SMICA at $N_{\text {side }}=2048$

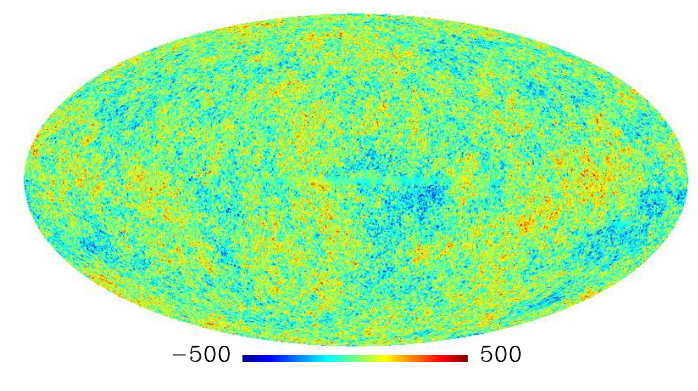

(c) Planck Minimal mask at $\mathrm{N}_{\text {side }}=2048$

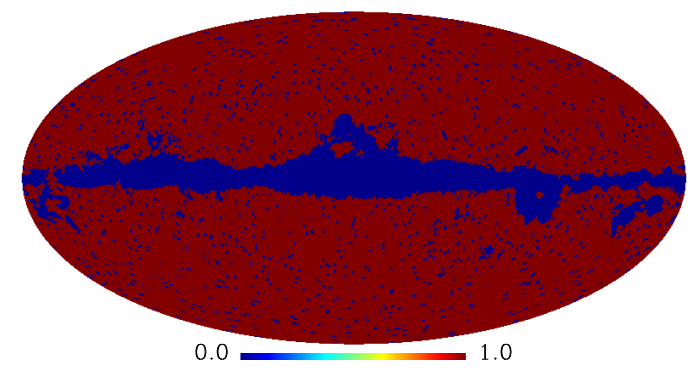

(b) NILC at $\mathrm{N}_{\text {side }}=2048$

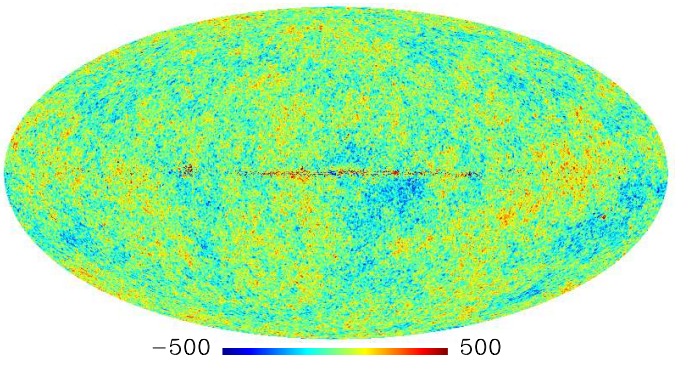

(d) X-ray Cluster template at $\mathrm{N}_{\text {side }}=2048$

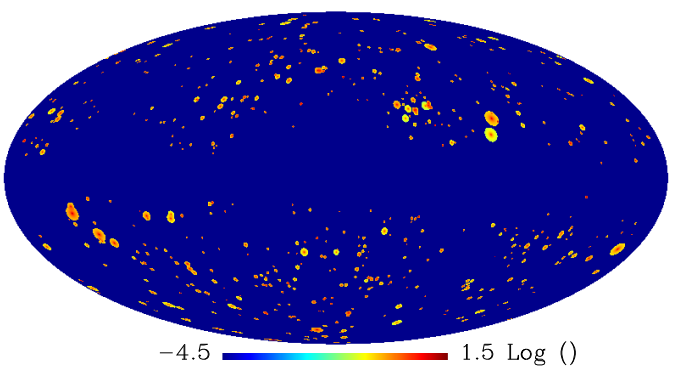

Figure 4.6: CMB maps SMICA (a) and NILC(b), galactic and point source mask (C) and cluster template (d) at resolution $N_{\text {side }}=2048$. The mask removes $\sim 33 \%$ of sky. The cluster TSZ template corresponds to $f(R)$ gravity with $\delta=-0.99, \zeta=0.1$ and polytropic index $\gamma=1.0$

At the center of each cluster the temperature anisotropy was measured over a disc of radius $r_{500} / 2$, where the $r_{500}$, radius of the cluster. Next, several consecutive measurements are obtained by averaging the signal over rings of width $r_{500} / 2$. Each data point corresponds to an angular separation averaged of the angular separation from the center of the pixels in each disc or ring. The square root of dispersion around the mean is about $0.1 r_{500}$ for the central disc and $\leq 0.05 r_{500}$ for the rings. The measured temperature profiles are dominated by the intrinsic CMB signal. To obtain a statistically significant signal that can be compared with the theoretical data, we 
stack the temperature anisotropies at the cluster locations. In Fig. 4.7a, we present the profiles measured in SMICA (diamonds) and NILC (triangles) for different cluster samples. In blue the stacked profile of the most luminous clusters, with $L_{X}[0.1-$ $2.4 \mathrm{keV}] \geq 2.5 \times 10^{44} \mathrm{erg} / \mathrm{s}$, in red the profile of the closest clusters, with $z \leq 0.16$, and in black the result for our full sample. The results from NILC and SMICA differ by less than $1 \%$. This implies that while the component separation techniques erase the frequency dependence of the TSZ effect, they do not distort the cluster profile and do not introduce systematics into our analysis. In Fig. $4.7 \mathrm{~b}$ we compare the profiles
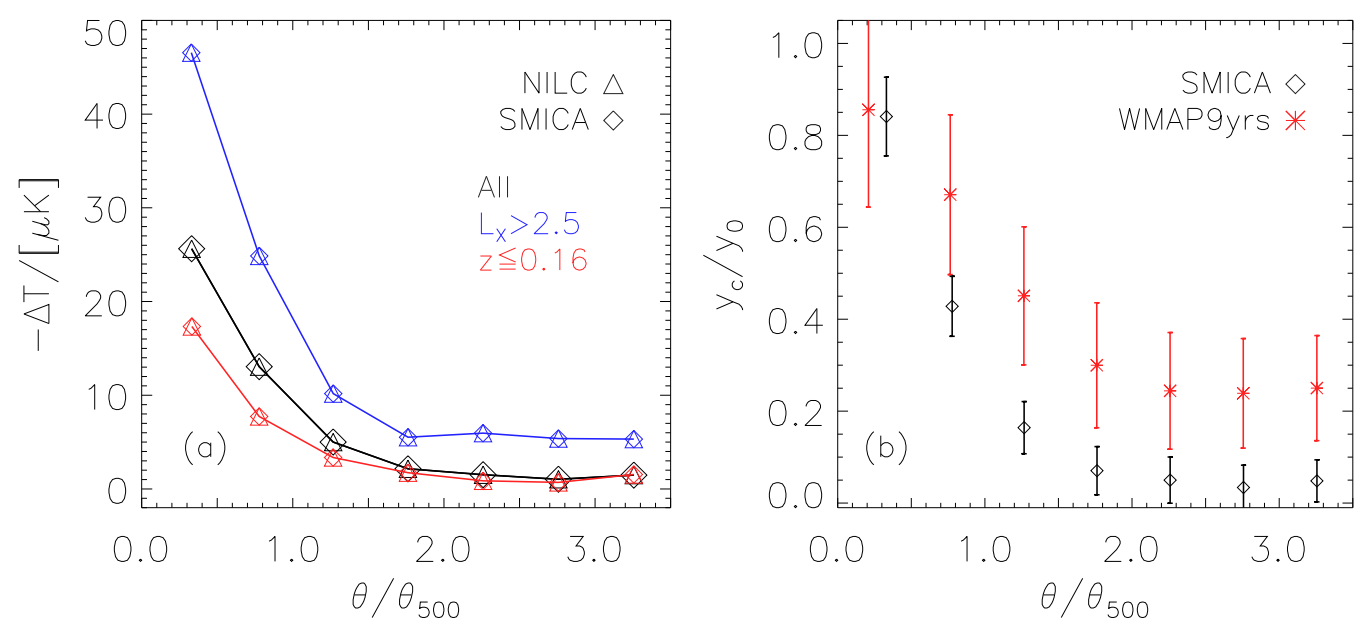

Figure 4.7: (a) Averaged temperature anisotropy profile for different cluster samples. Diamonds and triangles correspond to SMICA and NILC data. Profiles correspond to two cluster samples (blue and red solid lines) and to our full catalog (solid black line). (b) Comparison of the cluster profiles in SMICA (black diamonds) and WMAP 9yr W-band data (red asterisks) for our full cluster sample.

measured in SMICA (black diamonds) and in WMAP 9yr W-band data (red asterisks). The associated error bars are computed by evaluating 1,000 times the average profile at 579 random positions. When computing the simulations, we masked a disc of radius 80 arcmin around each cluster in our sample. WMAP and Planck show consistent profiles, the main difference being larger error bars and offset in WMAP emission at higher angular separations.

\subsubsection{The predicted thermal Sunyaev-Zeldovich profile}

To constrain an ETGs using data on galaxy cluster profiles, we need to compare the measured profiles with the theoretical expectation for the clusters in our sample. First, for cluster the scale length $r_{500}$ is computed using eq.(4.6) considered it to be exact. The pressure profile is computed solving eqs. (1.82), (4.24), (4.25) and (4.26) numerically as a function of $(\delta, L, \gamma)$. To account for the possibility that $L$, that describe the dependence of $f(R)$ on the scale of the system, might dependent on the physical properties of the cluster, we have considered two parametrization: (A) depends linearly 
on the size of the cluster, $L=\zeta r_{500}$ and (B) where $L=[0.1 ; 20] \mathrm{Mpc}$, is the same for all clusters.

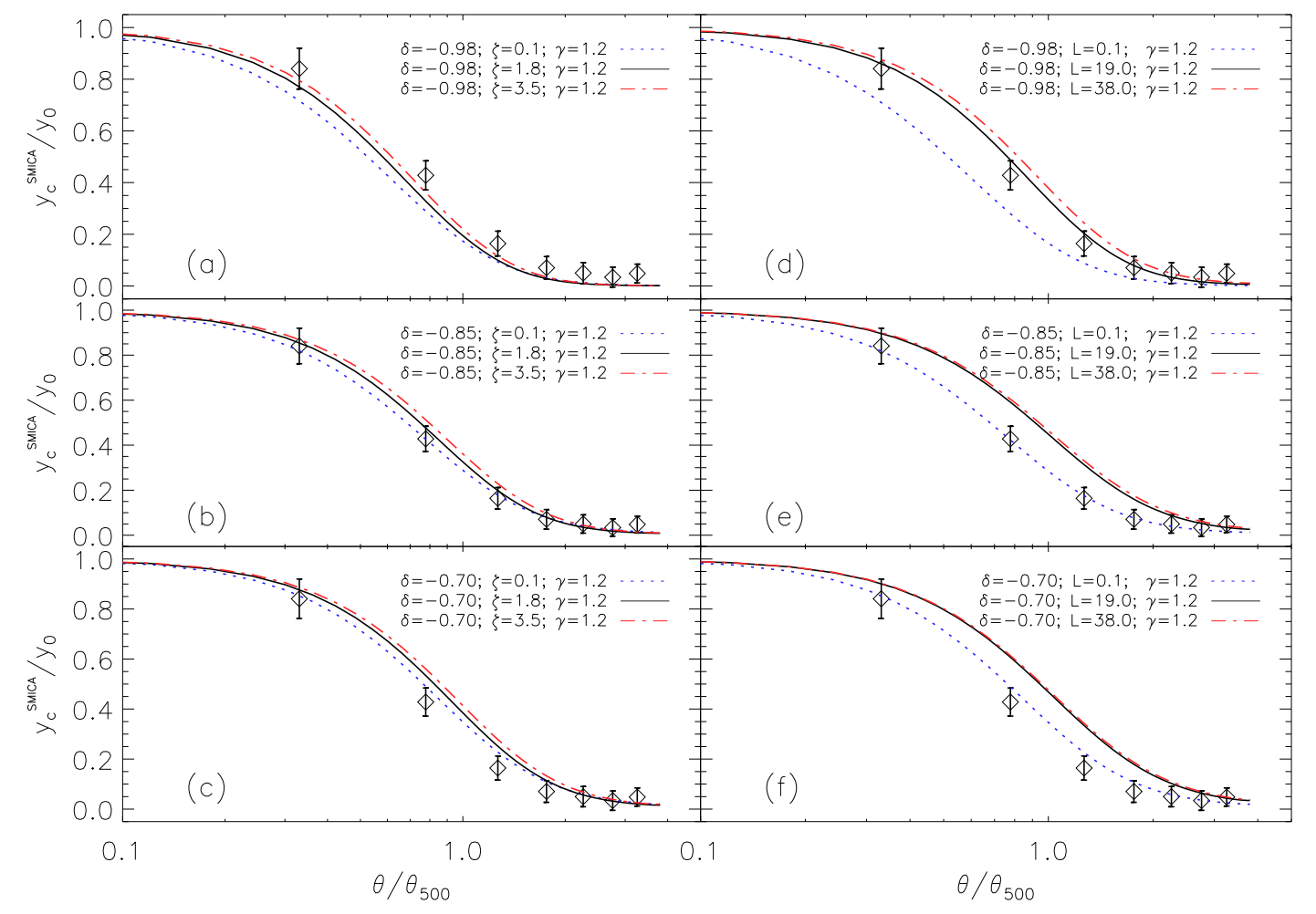

Figure 4.8: Pressure profiles of clusters in $f(R)$ gravity for different model parameters compared with the SMICA data. (a)-(c) correspond to the parametrization $L=\zeta r_{500}$ (Model A), while (d)-(f) correspond to $L=$ const for all clusters (Model B). Each model was averaged on the whole cluster sample, and normalized to the unity at the center.

To illustrate how the integrated pressure profiles depend on model parameters, in Fig. 4.8, we plot the profiles convolved with a Gaussian beam of 5 arcmin resolution. Our model only predicts the shape of the profile, but not its central anisotropy that needs to be fit to the data. This is not a limitation since, as the Planck Collaboration did not made publicly available the weights used to obtain the SMICA map, we can not determine the overall amplitude of the Comptonization parameter because the effective $g(\nu)$ is unknown. Then, we can only use the shape of the profile to constrain the model but not the amplitude. The data points are the profiles measured on SMICA, normalized to unity, with their corresponding error bars.

In Figs. 4.8a-c, $L$ is different for each cluster (Model A), and in Figs. 4.8d-f, $L$ is the same for all clusters (Model B). In order to avoid overcrowding the plots, we represent models with $\gamma=1.2$. Each panel shows the variation of $L$ for fixed $\delta$. For $L \geq 20 \mathrm{Mpc}$, profiles are essentially identical. This behavior could have been expected because $L$ is a correction of the Newtonian potential that becomes negligible for large $L$. 


\subsection{Estimation of the $f(R)$ parameters}

To find the best fit model, we generate cluster pressure profiles varying the parameters $(\delta, L, \gamma)$. The range of parameters was chosen on physical grounds. Since if $\delta<-1$ the potential is repulsive, and it diverges at $\delta=-1$, then $\delta=[-0.99,1.0]$. In model A we chose $L=\zeta r_{500}, \zeta=[0.1,4]$. The upper bound of the interval corresponds to the size of the cluster outskirts Planck Collaboration (2012). In model B, $L=[0.1,20] \mathrm{Mpc}$ is the same for all clusters. It ranges from the core radius to the mean cluster separation scale. The polytropic index was chosen in the range $\gamma=[1.0,1.6]$, that corresponds to an isothermal and adiabatic monoatomic gas. Profiles were constructed by taking 30 equally spaced steps in the three parameters. The three dimensional pressure profiles were integrated along the line of sight, and convolved with a Gaussian beam of 5 arcmin, the resolution of the SMICA map. For each set of parameters we compute the likelihood function $\log \mathcal{L}=-\chi^{2} / 2$ as

$$
\chi^{2}(\mathbf{p})=\Sigma_{i, j=0}^{N}\left(y\left(\mathbf{p}, x_{i}\right)-d\left(x_{i}\right)\right) C_{i j}^{-1}\left(y\left(\mathbf{p}, x_{j}\right)-d\left(x_{j}\right)\right),
$$

where $N=7$ is the number of data points, $y\left(\mathbf{p}, x_{i}\right)$ represents the averaged profile on all cluster sample, $d\left(x_{i}\right)$ is the SMICA average profile, and finally, $C_{i, j}$ is the correlation function between bins. The $\chi^{2}$ function depends on the parameters $\mathbf{p}=(\delta, L, \gamma)$. As indicated, error bars were computed choosing the same number of clusters, 579, than the data, but outside the mask and the known locations of clusters. $C_{i j}$ is the average correlation between bins averaged over all clusters and simulations.

In Figs. (4.9) and (4.10), we show the $68 \%$ and $95 \%$ confidence contours for the different pairs of model parameters of Model A and Model B, respectively. The value of model parameters that correspond to the best fit model are given in Table 4.3.
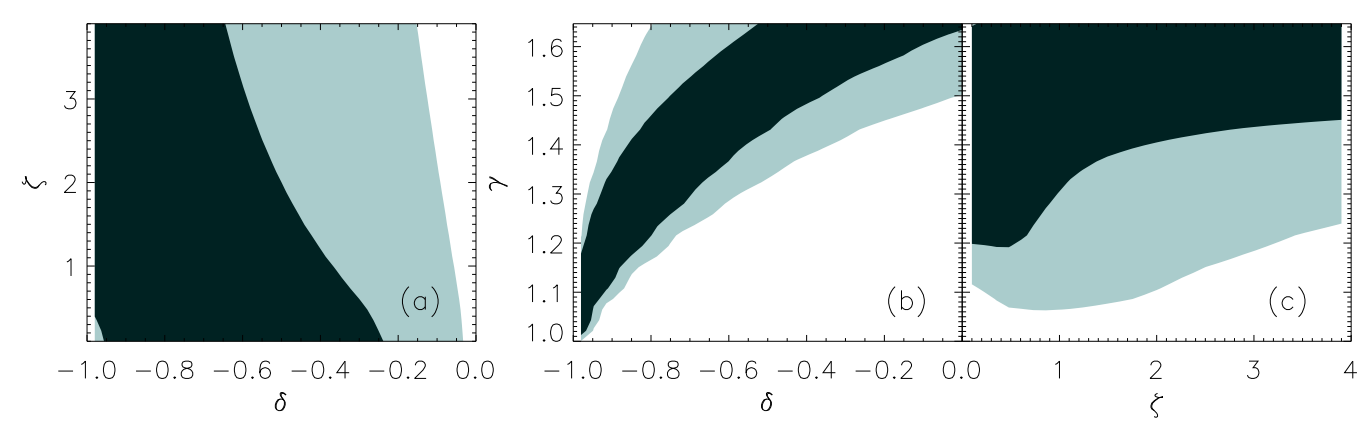

Figure 4.9: Confidence contours for pairs of parameters of Model A. Contours are at the $68 \%$ and $95 \%$ confidence level.

In Figs. 4.8a-c, $L$ is different for each cluster (Model A), and in Figs. 4.8d-f, $L$ is the same for all clusters (Model B). In order to avoid overcrowding the plots, we represent models with $\gamma=1.2$. Each panel shows the variation of $L$ for fixed $\delta$. For $L \geq 20 \mathrm{Mpc}$, profiles are essentially identical. This behavior could have been expected 

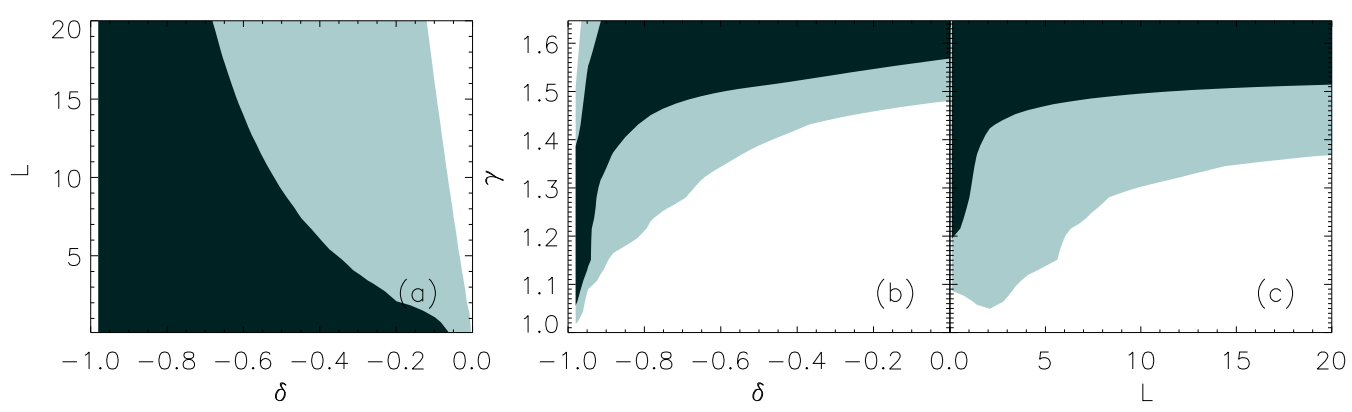

Figure 4.10: Same as in Fig. 4.9 for Model B.

because $L$ is a correction of the Newtonian potential that becomes negligible for large $L$.

\begin{tabular}{|l|cccccc|}
\hline \hline Model & $\delta$ & $\begin{array}{c}\mathrm{L} \\
(\mathrm{Mpc})\end{array}$ & $\gamma$ & $f_{0}^{\prime}$ & $\begin{array}{c}f_{0}^{\prime \prime} \\
\left(\mathrm{kpc}^{-2}\right)\end{array}$ & $\chi_{\text {dof }}^{2}$ \\
\hline \hline Model A $\left(L=\zeta<r_{500}>\right)$ & -0.98 & 1.12 & 1.07 & 0.02 & -0.003 & 0.25 \\
Model B & -0.98 & 1.91 & 1.07 & 0.02 & -0.001 & 0.25 \\
\hline Arnaud et al. (2010) & - & - & - & - & - & 1.38 \\
Planck Collaboration (2012) & - & - & - & - & - & 2.27 \\
Sayers et al. (2013) & - & - & - & - & - & 7.70 \\
$\beta(=2 / 3)$-model & - & - & - & - & - & 15.17 \\
\hline \hline
\end{tabular}

Table 4.3: $\chi^{2}$ per degree of freedom $\left(\chi_{d o f}^{2}\right)$ for the $\beta$-model, universal models with parameters given in Table 4.2, and for the two $f(R)$-parametrization considered in this work.

In Fig. 4.9 and Fig. $4.101 \sigma$ contours are cut by our physical limitations on $\delta$ and $\gamma$, and as a consequence, the 2D contours around the best fit model are not closed. Then, a confidence interval can not be derived from the data and only upper or lower limits can be given. At the $68 \%$ and $95 \%$ confidence levels, for model $\mathrm{A}$ those limits are $\delta<[-0.46,-0.10], \zeta<[2.5,3.7]$ and $\gamma>[1.35,1.12]$ and $\delta<[-0.43,-0.08], L<[12,19]$ Mpc and $\gamma>[1.45,1.2]$ for the Model B. Since the pressure profiles are very similar each other (see Fig. 4.8) we can not have strong constraints on model parameters. The data does not have enough statistical power to discriminate between models and parameterizations, as confirmed by low values of $\chi_{d o f}^{2}$. Even if our constraints are weak, we can extract useful information. First, the polytropic index is unconstrained by the data. Second, the characteristic scale length $L$ is very similar in both models (see Table 4.3), so we can not distinguish between parameterizations (A) and (B). Third, the data rules out $\delta=0$ at the $95 \%$ confidence level. The value $\delta \simeq 0$ corresponds to the standard Newtonian potential without DM. So our results indicate that baryons alone 
can not fit the cluster pressure profiles. Fourth, at the $1 \sigma$ level $L$ is compatible with zero, this condition corresponds to a gravitational field described by the Newtonian potential, but generated by a mass $M^{\prime}=M /(1+\delta)$. Since $M^{\prime} \gg M$, the gravitational field generated is analogue to one generated by a system that contains a large fraction of Dark Matter distributed like the baryonic gas. That is, either DM or a modified theory of gravity is required. Finally, in both models A and B we find the same correlation between the gravity parameters $L$ and $\delta$ that Sanders (1984) and Napolitano et al. (2012) found using spiral and elliptical galaxies, respectively. To fit the data larger values of $L$ require lower values of $\delta$. The central values of $\delta$ and $L$ are comparable with those derived from galaxies. In this context, the dynamics of galaxies and clusters can be described by ETGs with a single set of parameters.

To compare our model with the concordance model we have computed the $1 \mathrm{D}$ likelihood of each of the models given in Table 4.2 and their $\chi^{2}$ per degree of freedom. The results are given in Table 4.3. Like for WMAP (Atrio-Barandela et al., 2008), the $\beta$ model does not fit cluster pressure profiles. Since we do not fit model parameters to the data and just take the values of Table 4.2, the discrepancies between the three universal sets of parameters are not relevant since The results of ETGs fit rather better than the profiles on the concordance model. Therefore, ETGs could represent a viable description of the dynamics at the scale of clusters.

\subsection{Discussion and future perspectives}

In this chapter, we have explored the possibility to describe cluster pressure profiles in ETGs. Our model is based on introducing a Yukawa-like correction to the Newtonian potential in the weak field approximation of $f(R)$ gravity. We have fit the pressure profiles measured on the SMICA data to theoretical profiles generated for each cluster for each set of parameters. We have assumed that the baryonic gas is in hydrostatic equilibrium within the potential well of clusters. This hypothesis can only be tested using hydrodynamical simulations. However, as shown by Shi and Komatsu (2014), non-thermal pressure terms could be important in the description of cluster profiles. We lack a numerical study of these effects in ETGs, and therefore we could not include them in our analysis, somewhat weakening our conclusions. Within this limitation, models based on $f(R)$-gravity that do not require Dark Matter halos appear as a viable alternative to generalized NFW models. In other words, DM and ETGs models present equivalent descriptions of the cluster dynamics, and they could be discriminated only by some signature at fundamental scales, i.e. the discovery of new particles non-interacting at electromagnetic level, or the clear evidence of some new gravitational mode not related to General Relativity (Capozziello and De Laurentis (2011), Bogdanos et al. (2010)). 
In this study, we have not used frequency information because the foreground contamination Planck maps, publicly available, has not been removed. This limitation in the available information has increased our error bars widening our final contours than what they would be otherwise. A more detailed study would require using frequency information to benefit from the well know frequency dependence of the TSZ effect what would certainly lead to stronger constraints. 


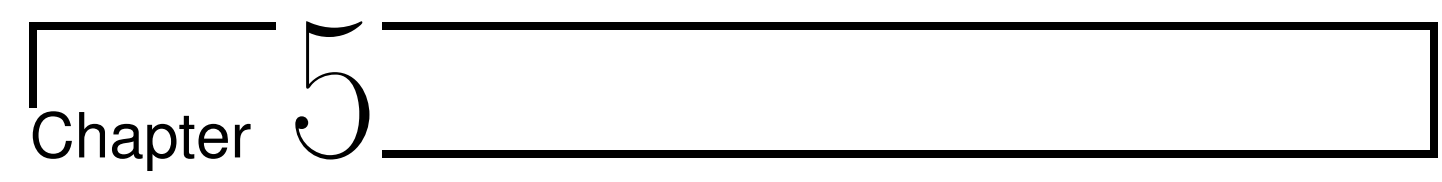

\section{Redshift evolution of the Cosmic} Microwave Background blackbody temperature

This study was carried out before the Planck Collaboration released their nominal maps in 2013; we used ancillary data such as masks, noise inhomogeneities from WMAP data release. The cosmological parameters correspond to WMAP 5year data.

\section{Contents}

5.1 Constraining redshift evolution of CMB temperature . . 83

5.2 Cluster templates and final maps . . . . . . . . 88

5.2.1 TSZ templates from X-ray selected clusters . . . . . . . 88

5.2.2 TSZ templates from N-Body hydrodynamical simulation . . . 89

5.2 .3 Final maps . . . . . . . . . . . . . . . . . . . . . . . 91

5.3 Data analysis and results . . . . . . . . . . 92

5.3.1 Ratio Method: analysis, systematics and results . . . . . . . 93

5.3.2 Fit Method: analysis, systematics and results . . . . . . . 96

5.4 Discussion and future perspectives $\ldots \ldots \ldots \ldots$

he adiabatic evolution of the Universe is one of the fundamental pillars of the
Big Bang paradigm. As a result of thermal equilibrium and photon number conservation in the early Universe, the CMB is a blackbody with a very high degree of accuracy. As a result, the blackbody temperature scales linearly in redshift, eq. (1.40). Its present temperature is $T_{0}=2.725 \pm 0.002 \mathrm{~K}$, measured with the FIRAS instrument in the COBE satellite (Fixsen et al. (1996), Mather et al. (1999)) and, more recently, using WMAP data (Bennett et al., 2003). However, deviations from adiabatic evolution would bring about a deep change in our understanding of physical theories at a fundamental level. Models like decaying vacuum energy density and/or 
gravitational 'adiabatic' photon creation predict the blackbody temperature scales nonlinearly as $T_{\mathrm{CMB}}(z)=T_{0}(1+z)^{1-\alpha}$ (Matyjasek (1995), Overduin and Cooperstock (1998), Lima et al. (2000), Puy (2004), Jetzer et al. (2011)). A value $\alpha \neq 0$ can be induced by a violation of the equivalence principle, if a fundamental constant such as fine-structure varies with $z$ (Murphy et al. (2003), Srianand et al. (2004)). Variation on the number density of photons could produced CMB distortions that are tightly constrain by FIRAS. It could be generated in decaying DE models (Freese et al. (1987), Lima and Trodden (1996), Jetzer et al. (2011)) or in models with axion-photon-like couplings (Jaeckel and Ringwald, 2010).

Currently there are two observational techniques that provide estimates of $T_{\mathrm{CMB}}(z)$ at redshifts $z>0$ :

(A) spectroscopic measurements of quasar spectra can help to identify excitation lines of high redshift clouds in equilibrium with CMB photons (Bahcall and Wolf (1968), LoSecco et al. (2001)). Unfortunately, the CMB is not the only source of excitation of the interstellar medium so quasar lines only provide an upper limit to the CMB blackbody spectra at the redshift of the cloud and large error bars $(\Delta T \geq 0.6 \mathrm{~K})$ (Meyer et al. (1986), Songaila et al. (1994a,b), Lu et al. (1996), Roth and Bauer (1999)). The first unambiguous measurement, with a considerably large error bar, was given by (Srianand et al., 2000). Recently, Noterdaeme et al. (2011) obtained a direct and precise measurement using the rotational excitation of carbon monoxide $(\mathrm{CO})$ in five systems. They constrained the deviation from linear scaling to be $\alpha=-0.007 \pm 0.027$ at $z \sim 3$.

(B) The second technique uses multi-frequency measurements of the TSZ effect, that is independent of redshift if the Universe evolves adiabatically (see eq. (1.41)), to estimate the CMB temperature at the location of clusters. Battistelli et al. (2002) probed $T(z)$ scaling relation using the spectral measurements of the SZ effect in two clusters of galaxies: COMA, at $z=0.0231$ and A2163 at $z=0.203$. They measured $\alpha=-0.16_{-0.32}^{+0.34}$. Luzzi et al. (2009), using multi-frequency measurements at different redshifts in the range $0.023 \pm 0.546$, showed $\alpha$ to be consistent with adiabatic evolution.

The two methods described above are complementary. They have different systematics and probe different redshifts. Spectroscopic measurements probe $z=[2 \div 4]$ while the SZ probes $z=[0 \div 1]$. While spectroscopic methods probe farther back in redshift, low-redshift measurements are important too. They probe the Universe when the expansion started to accelerate. In many theoretical models, there is a phase transition (Mortonson et al. (2009), Nunes et al. (2009)) which could be better traced with low redshift measurements.

In this chapter we will analyze if Planck data can constrain the evolution of the CMB black body temperature. The work presented here was previous to the first release of Planck data. Our analysis is based on simulations and we made use of 
WMAP mask to define our cluster sample and CMB simulations. For real CMB data, the effect of foregrounds has to be taken into account. It will depend both on the amplitude and shape of their power spectrum. Therefore, since Planck data were not publicly available, we did not include those contributions in our analysis. Foreground originate from different sources and are strongly dependent on frequency. Synchrotron and bremsstrahlung dominate the emission in the low frequency channels, dust, cosmic infrared background sources dominate at the highest frequencies while the $\mathrm{CO}$ has rotational lines at 100, 217 and $353 \mathrm{GHz}$. Because of their different origin, amplitude and distribution, foreground residuals are difficult to model. However, Galactic emissions do not correlate with clusters, and even if they can not be modeled accurately, in the real data we can expect to understand their effect on the value of $\alpha$ by carrying the analysis on cluster sub-samples in different regions of the sky. On other hand, diffuse and point-like radio sources can be found in cluster cores, in particular among the very X-ray luminous clusters. Again, this contribution could bias our estimate of $\alpha$ (Brunetti et al., 2007). Finally, the radio halos are found only in merging clusters (Cassano et al., 2010), whereas radio emission from individual cluster of galaxies is usually limited to the brightest ones and observed almost exclusively in relaxed systems. We aspect to understand their systematic by taking sub-sample of clusters.

In March 2013 the Planck Collaboration released its first year of data. Many of the effects simulated here can be studied in the released data. Maps of CO emission, dust, synchrotron, point sources and other foreground contributions have been derived from multi-frequency separation methods (Planck Collaboration, 2013g,e,q)) that offer the possibility to quantify their contribution in the real data. At the time we made this study the data was not available and we could only assume that those systematics could be evaluated using cluster subsamples, binning them by redshift and/or by galactic latitude.

\subsection{Constraining redshift evolution of Cosmic Microwave Background temperature}

Temperature anisotropies due to SZ effects are given by eq. (4.2), and their frequency dependence by the eq. (4.4). Under the Standard Cosmological Model they are redshift independent. However, if the Universe evolves non-adiabatically, the CMB temperature will vary in redshift and the most common functional forms studied in literature are: $T(z)=T_{0}(1+z)^{1+\alpha}$ (Lima et al., 2000) and $T(z)=T_{0}(1+\beta z)$ (LoSecco et al., 2001). In both parameterizations, $\alpha=0$ and $\beta=1$ correspond to adiabatic evolution. For redshifts $z \leq 0.7-1$ the differences between both models are small (see Fig. 5.1), so that we will restrict ourselves in studying the first model, that, hereafter, we will call $\alpha$-model. For this model, the reduced frequency changes with 
redshift as $x=x_{0}(1+z)^{\alpha}$, and the TSZ frequency dependence, $g(\nu)$, will depends on $\alpha, g(\nu)=g(\nu, \alpha)$. As a consequence, by measuring the frequency dependence of the TSZ effect at different redshifts we could constrain $\alpha$.

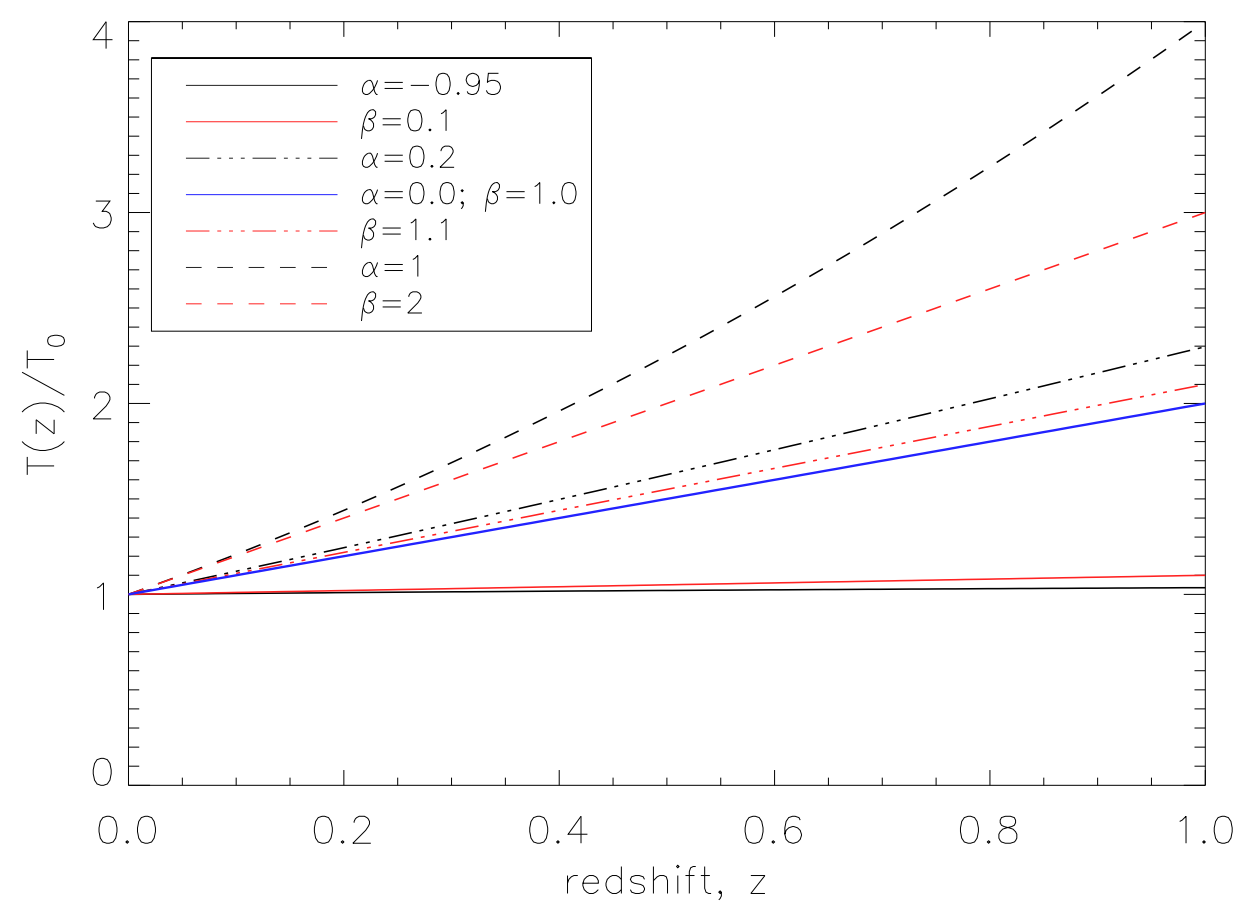

Figure 5.1: Parameterizations of the CMB temperature dependence with redshift. Black and red correspond to the $\alpha$ and $\beta$ parameterizations.

The function $g(\nu, \alpha)$ characterizes uniquely the TSZ contribution, but since, at each frequency, Planck channels are sensitive to a wide range of frequencies, then the spectral dependence of the TSZ measurements is not exactly $g(\nu)$ but must be convolved with the band width of the channels

$$
\bar{g}\left(\nu_{0}, \alpha\right)=\int_{0}^{\infty} g(\nu, \alpha) e^{\left(\nu-\nu_{0}\right)^{2} / 2 \sigma_{\nu}^{2}} d \nu
$$

Hereafter, for sake of simplicity, $g(\nu, \alpha)$ will refer to the frequency averaged dependence given in eq (5.1).

As shown in Fig. 4.1b, for an adiabatic evolution the TSZ is null at $\nu \simeq 217 G H z$. Measuring the zero cross frequency of clusters at different redshifts, deviations from adiabatic evolution could be measured (Fabbri et al., 1978). An alternative technique uses ratios of the TSZ anisotropies at different frequencies (Rephaeli (1980), Luzzi et al. (2009)). By taking ratios, the dependence on gas temperature $T_{e}$ and on the 
cluster profile is removed

$$
\frac{\left(\frac{\Delta T\left(\nu_{1}, z^{*}\right)}{T_{\mathrm{CMB}}}\right)}{\left(\frac{\Delta T\left(v_{2}, z^{*}\right)}{T_{\mathrm{CMB}}}\right)}=\frac{g\left(\nu_{1}\right)}{g\left(\nu_{2}\right)}
$$

Nevertheless, the analysis becomes more complicated since the distribution of temperature ratios is highly non-Gaussian (Luzzi et al., 2009). For non-adiabatic evolution, the ratios will depend on $\alpha$ as

$$
R\left(\nu_{1}, \nu_{2}, \alpha\right)=\frac{g\left(\nu_{1}, \alpha\right)}{g\left(\nu_{2}, \alpha\right)}
$$

For this ratio to give accurate measurements of $\alpha, \mathrm{CMB}$ temperature anisotropies, foreground residuals have to be small compare with the cluster anisotropy. In Fig 5.2 we plot the frequency dependence of the ratio (Fig 5.2a) and zero cross frequencies (Fig 5.2b) for different values of $\alpha$. In Fig 5.2a, the solid line represents the redshift
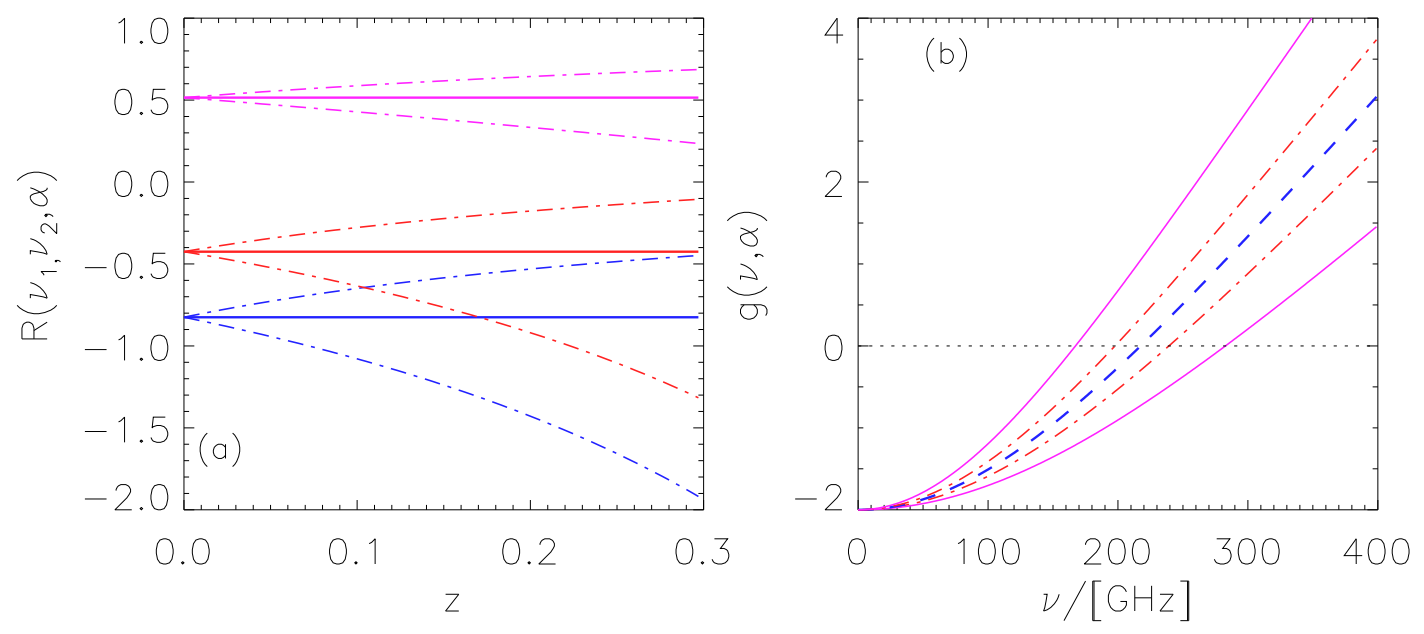

Figure 5.2: (a) variation of the ratio $g(\nu) / g(353 G H z)$ as a function of redshift for $\nu=143 \mathrm{GHz}$ (top set of curves), $\nu=100 \mathrm{GHz}$ (middle set) and $\nu=44 \mathrm{GHz}$ (lower set). The solid line correspond to adiabatic evolution, $\alpha=0$ and the dot-dashed lines $\alpha=1,-1$. (b) Spectral dependence for $\alpha=-1,1$ for two clusters located at $\mathrm{z}=0.3$ (solid lines) and $\mathrm{z}=0.1$ (dot-dashed line). The dashed lines correspond to adiabatic evolution and is the same for a cluster located at any redshift. The zero amplitude of the TSZ effect is indicated by the dotted line.

independent ratio $(\alpha=0)$; the dot-dashed lines bound the region where $\alpha=-1,1$. From top to bottom, the ratios are $R(\nu, 353 G H z, \alpha)$ with $\nu=143,100,44 \mathrm{GHz}$. In Fig $5.2 \mathrm{~b}$, we plot the spectral dependence $g(\nu, \alpha)$ for adiabatic evolution $(\alpha=0$, dashed line) and $\alpha=-1,1$ for a cluster at redshift $z=0.1$ (dot-dashed line) and $z=0.3$ (solid line).

However, the intrinsic CMB fluctuations are non-negligible. They dominate over the TSZ signal except for the most massive clusters, biasing the redshift dependence of $g(\nu, \alpha)$. For example, if the CMB is dominant then the ratio will be close to the 
unity. Furthermore, hot/cold CMB spots at a cluster shifts the zero cross frequency to frequencies lower/higher than $217 \mathrm{GHz}$. Moreover, other components could also complicate the analysis, such as instrument noise or foreground residuals. On other hand, the magnitudes $g(\nu, \alpha)$ and $R\left(\nu_{1}, \nu_{2}, \alpha\right)$ derived from the data will not follow the curves of Fig. 5.2. We have proposed to degrade the $217 \mathrm{GHz}$ channel angular resolution (the highest of the PLANCK channels) to that of the other channels to remove the intrinsic $\mathrm{CMB}$ fluctuations. In Fig 5.3a, we plot the ratio of the CMB removed maps at different frequencies

$$
R_{[-217 G H z]}\left(\nu_{1}, \nu_{2}, \alpha\right)=\frac{g\left(\nu_{1}, \alpha\right)-g(217 G H z, \alpha)}{g\left(\nu_{2}, \alpha\right)-g(217 G H z, \alpha)}
$$

and in Fig 5.3b we represent the TSZ spectral dependence

$$
g_{[-217 G H z]}(\nu, \alpha)=g(\nu, \alpha)-g(217 G H z, \alpha) .
$$

The lines follow the same conventions than in Fig 5.2.
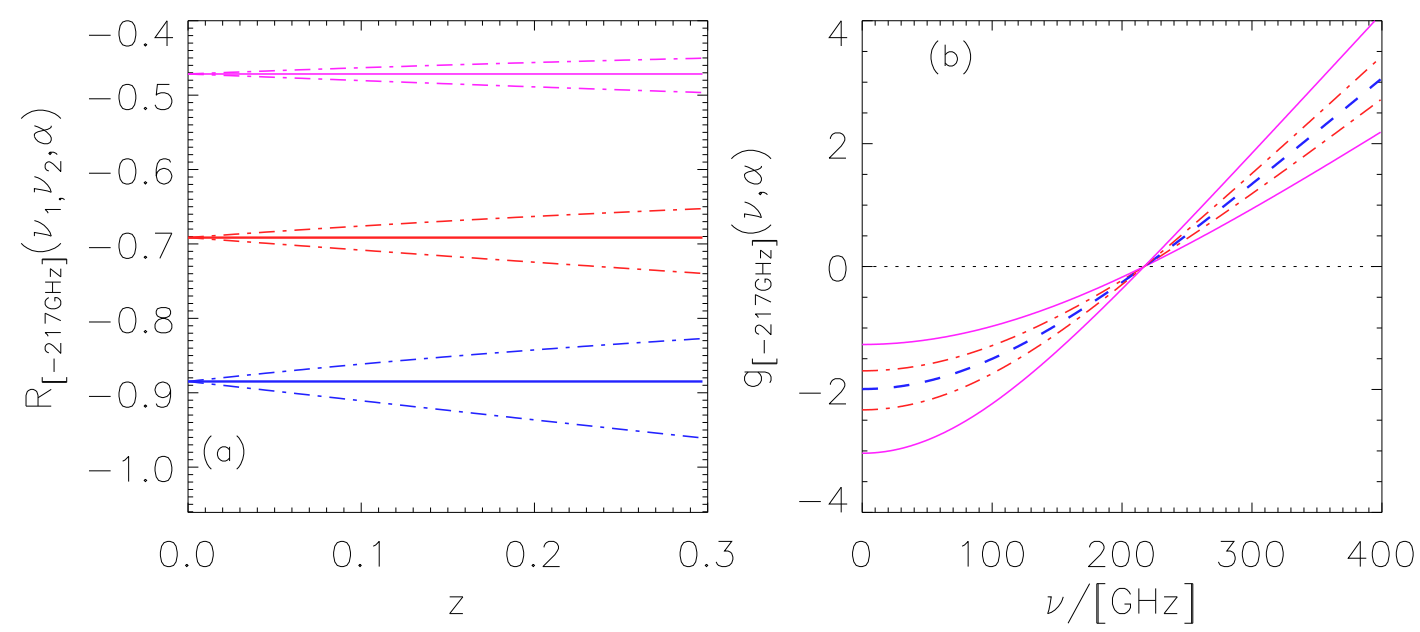

Figure 5.3: (a) Ratio of $r(\nu, 353 G H z)=[g(\nu)-g(217 G H z)] /[g(354 G H z)-g(217 G H z)]$ for $\nu=143,100$ and $44 \mathrm{GHz}$, and (b) Spectral dependence of $g(\nu)-g(217 G H z)$. Curves follow the same convention as in Fig. 5.2.

The estimator $R_{[-217 G H z]}$ has a weaker dependence on $\alpha$ than $R$. To make the test possible, a large number of clusters at high redshift are needed. Furthermore, the zero cross frequency is now independent of $\alpha$. In order to constrain $\alpha$, we need to fit the spectral shape to the data. This makes the method more complicated since we do not measure $g(\nu, \alpha)$ directly on CMB maps. The quantities that we measure are temperature anisotropies $\Delta T(\hat{n})=T_{0} y_{c} g(\nu, \alpha)$, so that, for example, if we consider a cluster at $z=0.1$, and $\alpha=-1,+1, g_{[-217 G H z]}(\nu, \alpha)$ changes by a $50 \%$ in the range of frequencies probed by PLANCK. But, if the Comptonization parameter $y_{c} \propto \int T_{X} d \tau$ was uncertain by the same factor, the subtracted maps $\Delta T_{[\nu,-217 G H z]}(\hat{n})=\left[\Delta T_{\nu}(\hat{n})-\right.$ $\left.\Delta T_{217 G H z}(\hat{n})\right]$ would be unable to constraint $\alpha$. To use the results of Fig $5.3 \mathrm{~b}$ we need 
to determine the cluster Comptonization parameter from X-ray data, so we can predict the amplitude of the $y_{c}$-map, and by measuring $\Delta T(\hat{n})$ at the PLANCK frequencies, constrain $\alpha$.

We simulated data by generating random maps that include a cosmological CMB signal and instrumental noise noise. To these maps we added the TSZ signal of a cluster template generated (i) from the X-ray data of a real cluster catalog and (ii) from a N-body simulation of clusters. The TSZ signal we use as reference is the adiabatic model $(\alpha=0)$. We design pipelines and we characterize their performance by computing $\alpha$ and comparing with the input data of $\alpha=0$.

For the X-ray selected cluster template we do not have information about the velocity field to include the KSZ contribution, so only the TSZ component was added, while for the N-Body simulated clusters both TSZ and KSZ contributions were included. In this way, by testing the performance of our methods in cluster templates constructed from an N-Body simulation, we can obtain a better understanding of all uncertainties and systematics. Our pipelines are:

- Pipeline (A): first, we assume that a component separation method will remove the intrinsic CMB contribution, leaving some residual. In here we adopted the filter described in Kashlinsky et al. (2009) to create our CMB residual template. This filter is designed to remove the primary CMB fluctuations from the concordance $\Lambda \mathrm{CDM}$ model by minimizing the mean squared deviation of the $\mathrm{CMB}$ measurements from the noise $\left\langle\left(\delta T_{C M B}-\text { noise }\right)^{2}\right\rangle$. It generates CMB residuals that are homogeneously distributed in the sky. In this respect we are being conservative; the true residuals are likely to be smaller away from the galactic plane (see Planck HFI Core Team 2011b, Figure 39), fact that could permit to estimate the effect of the residuals by analyzing cluster subsamples selected according to galactic latitude. Together with $\mathrm{CMB}$ residuals, the map will contain instrumental noise, KSZ and TSZ and some unknown level of foreground residuals. The CMB residuals are the same at all frequencies while the noise realization is different for each frequency. To simplify our simulations we take the amplitude of the CMB residuals to be $\left\langle\Delta T_{c m b, r e s}^{2}\right\rangle=1,10 \mu \mathrm{K}$. The temperature anisotropies at each pixel is

$$
\Delta T_{A}(\nu)=y_{c} g(\nu, \alpha=0) \pm \sigma_{\text {noise }}^{A}(\nu) \pm \Delta T_{C M B \text { res }} .
$$

- Pipeline (B): We use the $217 \mathrm{GHz}$ channel to remove exactly CMB and KSZ signals. At each frequency the resolution of the $217 \mathrm{GHz}$ is downgraded to the resolution of the channel before subtracting it. We checked the power spectrum of the final maps was a pure white noise, with a slightly larger variance, $\sigma_{\text {noise }}^{B}(\nu)$, 
due to the noise of the the $217 \mathrm{GHz}$ channel. At each pixel we measure

$$
\Delta T_{B}(\nu)=y_{c} g_{[217 G H z]}(\nu, \alpha=0) \pm \sigma_{\text {noise }}^{A}(\nu)
$$

All maps were constructed using HealPix package (Górski et al., 2005) with resolution $N_{\text {side }}=1024$.

\subsection{Cluster templates and final maps}

Let us briefly describe the construction of the TSZ templates, the simulated final maps and the implementation of our pipelines.

\subsubsection{TSZ templates from X-ray selected clusters}

We have used the X-ray cluster catalog described in Sec. 4.3.1, and since the minimal Planck mask (Fig. 4.6c) was not publicly available when this work was carried out, we perform our analysis on the 623 clusters that were outside WMAP Kp0 mask that is shown in Fig. 5.4.

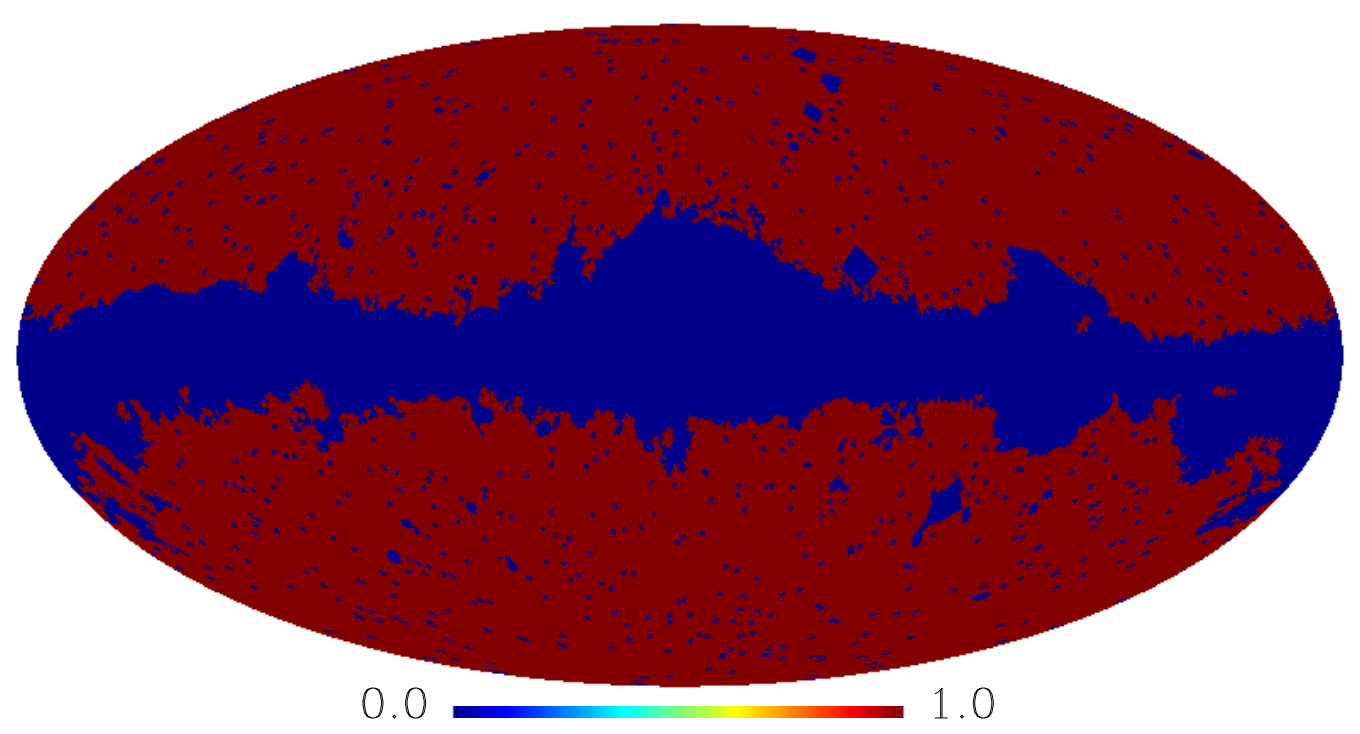

Figure 5.4: WMAP 7 yrs data: Kp0 Mask.

From the X-ray data we can predict the electron pressure profile assuming a cluster model for the gas. We assumed two models: the $\beta$-profile (Sect. 4.1.2) and the universal cluster pressure profile (Sect. 4.1.4). Then, templates are convolved with the antenna of each channel (the relevant data is given in Table 1.3) and multiplied by $g(\nu, \alpha=0)$ (our reference model) to generate TSZ templates. As an example, the resulting templates are shown in Fig. 5.5 for the $70 \mathrm{GHz}$ and $353 \mathrm{GHz}$ channels. 

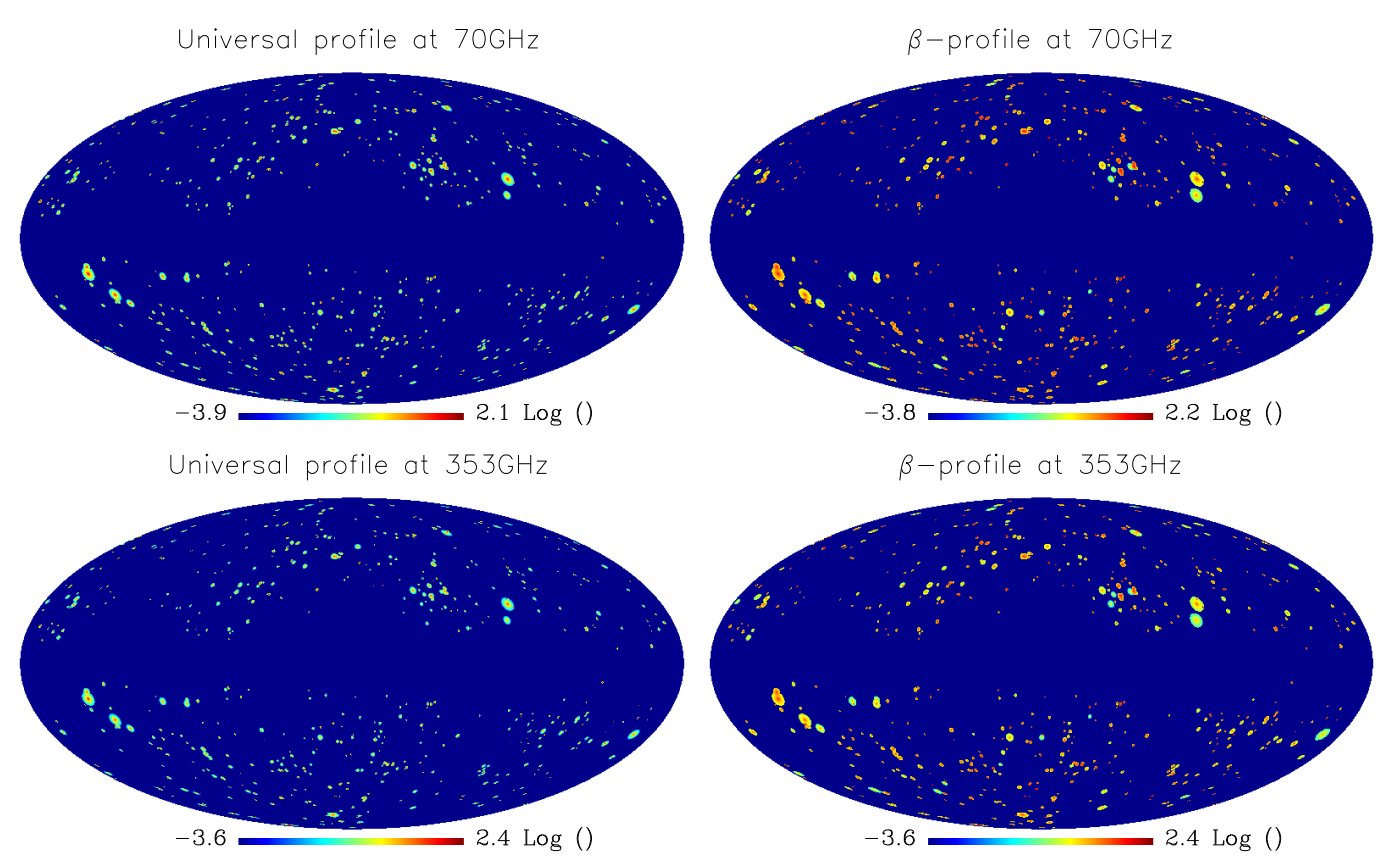

Figure 5.5: TSZ template containing the profile of 623 clusters outside WMAP Kp0 mask. The maps are represented in a logarithmic scale for clarity.

In Fig. 5.6a we represent the pressure profile of a real cluster, integrated along of line of sight for the $\beta=2 / 3$ (solid line) and universal pressure (dashed line) profiles, convolved with the antenna of the $44 \mathrm{GHz}$ map. The cluster is located at $z=0.094$ and has a mass $M_{500}=2.410^{14} \mathrm{~h}^{-1} \mathrm{M}_{\odot}$ and $r_{500}=746 \mathrm{~h}^{-1} \mathrm{kpc}$. In Fig. $5.6 \mathrm{~b}$ we plot the central value of Comptonization parameter $y_{c}$ of the 623 clusters considered in this study. The solid line represents the linear regression fit to the data and from this regression we obtain the following scaling: $y_{c}=24.5\left(M_{500} / 10^{14} \mathrm{~h}^{-1} \mathrm{M}_{\odot}\right)^{1.35}$.

\subsubsection{TSZ templates from N-Body hydrodynamical simulation}

The templates constructed from X-ray-selected clusters assume clusters to be spherically symmetric and relaxed. The hydro-simulated TSZ and KSZ templates contain clusters with different dynamical states (relaxed, merging systems, etc.), shapes and ellipticities. The TSZ and KSZ signals will be obtained by integrating along the line of sight so the projection effect due to low mass clusters and groups are included. Therefore, these templates are very well suited to study the effect of these systematics in the determination of $\alpha$.

The simulations we will used were constructed for the pre-launch Planck Sky model and are described in Delabrouille et al. (2013). The simulated cluster catalog contains the masses and radii and redshifts of all the clusters. The mass is $M_{200}$, the mass on a sphere of radius $r_{200}$, where the overdensity is 200 times the critical density; clusters have redshifts $z \leq 0.25$. The maps contains the SZ signals integrated along the 

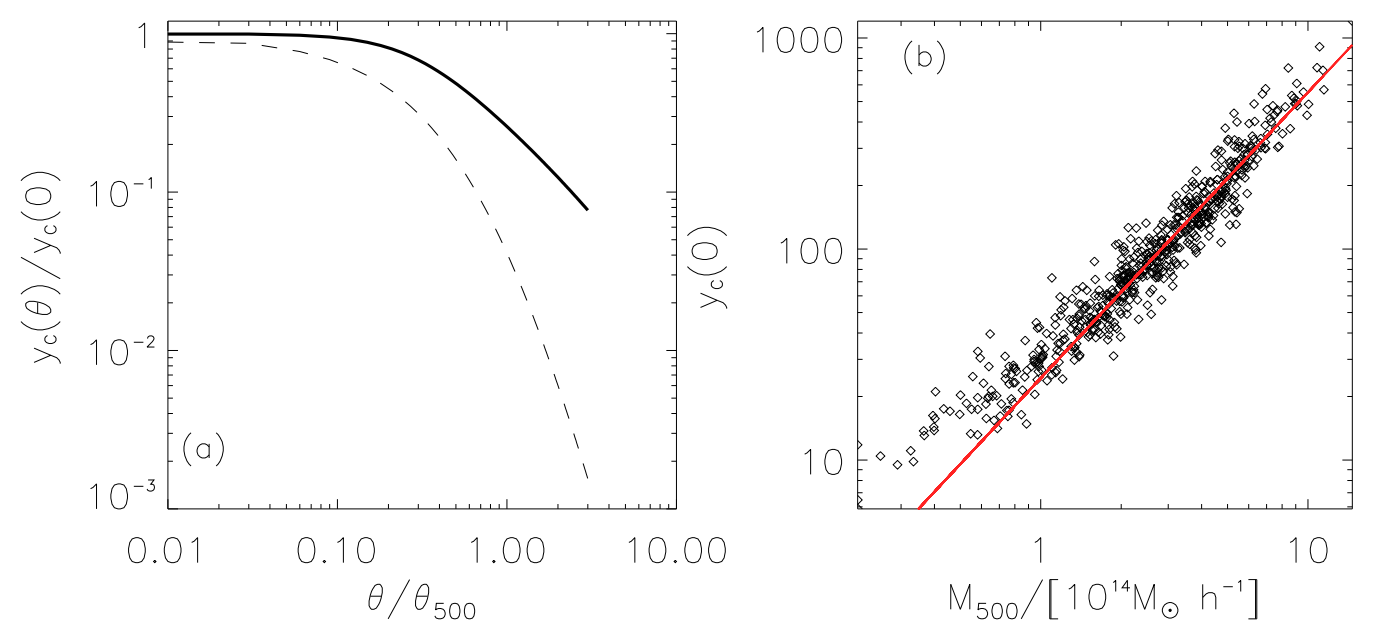

Figure 5.6: (a) Pressure profile integrated along the line of sight and convolved with antenna beam at $44 \mathrm{GHz}$ channel for a cluster of $M_{500}=2.410^{14} \mathrm{~h}^{-1} \mathrm{M}_{\odot}$ at $z=0.094$. The solid line corresponds to the $\beta=2 / 3$ profile and the dashed line to the universal profile. (b) Central value of the Comptonization parameter for all the clusters in our sample. The solid line corresponds to the linear regression fit to the data.

line of sight, and computed combining full hydrodynamic simulations following the "box stacking method". Templates are constructed in layers of co-moving thickness $100 \mathrm{~h}^{-1} \mathrm{Mpc}$ from the outputs of N-body hydrodynamical simulations. The light-cone integrations are carried out using the formula in da Silva et al. (2000, 2001). The range of redshifts was divided in seven layers, the innermost layer includes the local constrained simulation of Dolag et al. (2005), whereas all the other layers were produced from gas snapshots of the $\Lambda$ CDM simulation in De Boni et al. (2011). Both these simulations include explicit treatment for gas cooling, heating by UV, star formation, and feedback processes. The templates, in log-scale, are shown in Fig. 5.7
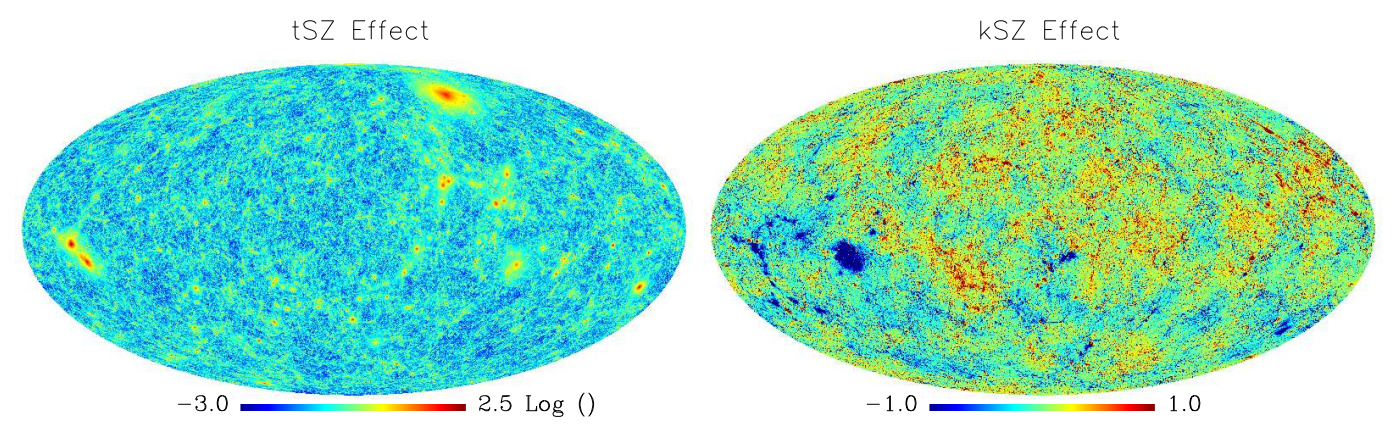

Figure 5.7: TSZ and KSZ templates from N-Body hydrodynamical simulations (Delabrouille et al., 2013).

To compare with our X-ray template, we express cluster properties in terms of $r_{500}$ instead of $r_{200}$. In Fig. 5.8a we present the mass and redshift distribution of our 623 real clusters. The solid red line is our selection function. In Fig. 5.8b we show all the clusters in the simulations that verify the selection criteria of the data. The linear 
patterns corresponds to the same cluster at different redshifts. In Fig. 5.8c,d we plot the mass and redshift distribution of all clusters in our simulation (solid line) that fulfill the selection criteria. The dashed line shows the same distributions of the X-ray clusters. For a better comparison, the histograms were normalized to unity. The main difference between the two samples is that there are 22 clusters in our catalog with redshifts larger than $\mathrm{z}=0.2$. Of all the simulated clusters, we selected randomly 623 to construct the simulated template.
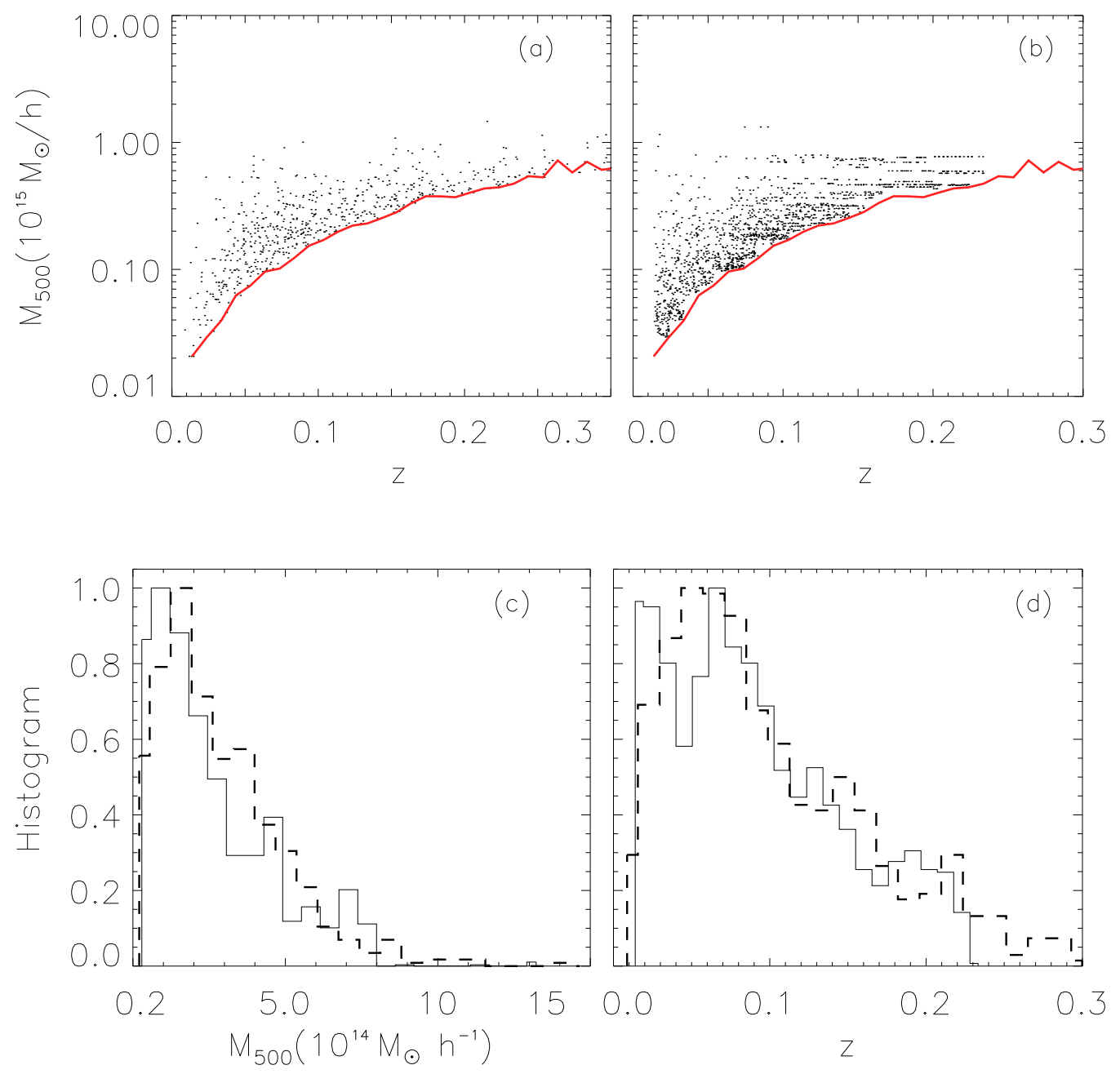

Figure 5.8: Comparison between X-Ray and simulated clusters. (a) Mass of X-ray clusters is shown as function of redshift; the selection function is represented by a thick red line. (b) Mass function of simulated clusters selected according to the selection function of the real clusters. (c) Mass and (d) redshift distribution of all the selected simulated clusters (solid line) and of the X-ray clusters (dashed line)

\subsubsection{Final maps}

For realistic simulations, noise and $\mathrm{CMB}$ anisotropies have to be added to the SZ templates. Noise maps were constructed as homogeneous and uncorrelated white noise 
by assuming the noise levels given in Table 1.3. For the cluster templates generated using X-ray data we have included relativistic corrections using the analytic formula derived by Itoh et al. (1998) and Nozawa et al. (1998). The KSZ template was added to the N-Body hydrodynamical simulation in order to understand how much it will affect the final constraint on the redshift evolution of the CMB temperature. However, it was not added to the template constructed using the X-ray data since individual cluster peculiar velocities have not been measured yet.

\subsection{Data analysis and results}

Let us now discuss how well our pipelines (A) and (B) recover the input value $\alpha=0$. Our signal will be the temperature anisotropy averaged over a disc of fixed radius at each cluster location. Each disc encompasses $N_{\text {pixel }}$. The white noise scale as $\sqrt{N_{\text {pixel }}}$ but other components like intrinsic CMB, foreground residuals or $(1 / f)$ noise do not. These latter contributions will bias our estimate of the the Comptonization parameter. Then, in each pipeline we implement the ratio and the fit method and we will discuss their relative merits. To simplify the notation, let the index $I=(A, B)$ represent the pipeline and let us redefine $g_{A}=g(\nu, \alpha), R_{A}=R\left(\nu_{1}, \nu_{2}, \alpha\right), g_{B}=g_{[-217 G H z]}(\nu, \alpha)$ and $R_{B}=R_{[-217 G H z]}\left(\nu_{1}, \nu_{2}, \alpha\right)$. The temperature anisotropy averaged over a disc is

$$
\left\langle\Delta T_{I}\left(\nu_{1}\right)\right\rangle=\bar{y}_{c} g_{I}\left(\nu_{1}\right) \pm \frac{\sigma_{\text {Noise } \nu_{1}}^{I}}{\sqrt{N_{p i x}}},
$$

where $N_{p i x}$ is the number of pixels occupied by the cluster. There will be an extra KSZ component for simulated cluster templates.

We tested the ratio and fit methods using the template constructed from simulations and we repeated the analysis using the template of X-ray selected clusters. We found no significant differences from the results computed using both templates. Therefore, projection effects, kSZ contributions, cluster dynamical state and deviations from spherical symmetry are averaged out over such a large cluster sample. Indeed, those effects are relevant when analyzing observations with less number of clusters (Battistelli et al. (2002), Luzzi et al. (2009)) but they are not relevant here. We found that the fit method constraints $\alpha$ equally in both pipelines but the ratio method performs better in pipeline B.

In order to test the contribution of different components, we performed the analysis in three mass bins of equal number $(\sim 208)$ of clusters, and three redshift bins, also with the same number of clusters. For each subsamples $\alpha \pm \sigma_{\alpha}$ was constrained by computing the likelihoods for different frequencies in order to determine the subset with the largest statistical power. Models were constructed subdividing the interval $\alpha=[-1,1]$ in 2001 steps, equally spaced, and performing 1,000 Monte-Carlo simulations for each cluster template, method and pipeline. Concerning the ratio method, we will present results 
only on simulated clusters since the results do not change with template. For the fit method we will present the analysis on simulated and real clusters, and we will show that X-ray information can improve the constraint on $\alpha$.

\subsubsection{Ratio Method: analysis, systematics and results}

In this method we estimate $\alpha$ from the ratios at different frequencies of the average temperature anisotropies on disc of fixed radius. Maps are brought to a common resolution before taking the ratio so there are no effect due to differences in the antenna beam. As discussed in Luzzi et al. (2009), the ratio of two Gaussian distributed variables is not a Gaussian. The probability distribution of the ratios, $\mathcal{P}_{j}\left(R_{I}\right)$, is

$$
\begin{aligned}
\mathcal{P}_{j}\left(R_{I}\left(\nu_{1}, \nu_{2}, \alpha\right)\right) & =\frac{1}{2 \pi \sigma_{1} \sigma_{2}} \times \\
& \times \int_{-\infty}^{\infty} x \exp \left(-\left[\frac{\left(x-\delta_{1}\right)^{2}}{2 \sigma_{1}^{2}}+\frac{\left(x R_{I}\left(\nu_{1}, \nu_{2}, \alpha\right)-\delta_{2}\right)^{2}}{2 \sigma_{2}^{2}}\right]\right) d x^{2}
\end{aligned}
$$

where $\delta_{j}\left(\nu_{i}\right)=\left\langle\Delta T_{j, I}\left(\nu_{i}\right)\right\rangle$ is the average temperature anisotropy, $\sigma_{i}$ is the Gaussian error associated to $j$-th cluster in the frequency channel $i$ and $R_{I}\left(\nu_{1}, \nu_{2}, \alpha\right)$ is the theoretical estimation of the ratio $\delta_{j}\left(\nu_{1}, \alpha\right) / \delta_{j}\left(\nu_{2}, \alpha\right)$. A few examples are given in Fig $5.2 \mathrm{a}$ and $5.3 \mathrm{a}$. The best fit of $\alpha$ is given by the maximum of the likelihood function

$$
-2 \log \mathcal{L}=\sum_{\nu_{1}, \nu_{2}} \sum_{j=1}^{N_{c l}} \log \left[\mathcal{P}_{j}\left(R_{I}\left(\nu_{1}, \nu_{2}, \alpha\right)\right)\right]
$$

Luzzi et al. (2009) showed that the ratio of two distribution is biased by the error on the denominator. When in the denominator $\left\langle\Delta T_{\nu_{2}}\right\rangle$ is close to zero, the ratio is very large. Although the probability $\mathcal{P}$ of a large ratio is very small (see eq. 5.3.1) to reduce this bias, in pipeline $A$ the denominator is reserved for the data with the smallest noise, 100 and $143 \mathrm{GHz} ; 217 \mathrm{GHz}$ is excluded from the denominator because the TSZ signal is zero at that frequency. In pipeline $B$ the biased is reduced by rejecting clusters for which $\left\langle\Delta T_{\nu_{2}}\right\rangle \leq 0.1 \sigma_{\text {noise }, \nu_{2}} / \sqrt{N_{\text {pix }}}$. In eq. (5.10), the errors $\sigma_{\text {ratio }, i}^{I}$ are computed for each cluster as the rms deviation of 1,000 simulations of the ratio $\left\langle\Delta T_{I}\left(\nu_{1}\right)\right\rangle /\left\langle\Delta T_{I}\left(\nu_{2}\right)\right\rangle$. The TSZ component is fixed to the value at the cluster location, and the noise is drawn from a Gaussian distribution with zero mean and variance $\left(\sigma_{\text {noise }, \nu}^{I}\right)^{2} / N_{p i x, i}$.

In the ratio method we do not need the cluster profile or any other observational properties. Templates of X-ray or simulated clusters will produce the same constraints on $\alpha$. Then, we shall only present the results for simulated clusters since for these templates include many real effects that could bias the results, like projection effects, cluster sphericity, relaxed and interacting clusters, etc, not included in the X-ray cluster template. 


\section{Results for a template of simulated clusters}

In Fig. 5.9 we represent the likelihood function of the ratio method for pipeline $A$, with CMB residuals of amplitude $\Delta T_{C M B \text {,res }}=1 \mu \mathrm{K}$. In Fig. 5.9 (a) the likelihood is computed for three redshift bins, marginalizing over 10 frequency ratios. The redshift bins were selected so they would have the same number of clusters: $z=[\leq 0.11,0.11-$ $0.17,>0.17$, represented in the figure by the black dot-dashed, red dashed and blue solid lines. As expected, the high redshift bin provides the strongest constraint $\alpha=$ $-0.052 \pm 0.011$. In Fig. 5.9(b) cluster are binned by mass. Again, the intervals are chosen to have the same number of clusters: $M_{500} \leq 2 \times 10^{14} \mathrm{~h}^{-1} \mathrm{M}_{\odot}$, (black dot-dashes), $M_{500}=2-3.6 \times 10^{14} \mathrm{~h}^{-1} \mathrm{M}_{\odot}$ (red dashes) and $M_{500} \geq \times 3.6 \times 10^{14} \mathrm{~h}^{-1} \mathrm{M}_{\odot}$ (blue solid line). For the most massive clusters $\alpha=-0.028 \pm 0.013$. In Fig. 5.9(c) we show the the full likelihood for one single realization (black dashed line) and also for 1,000 realization of the sky (histogram). The blue thick solid line is a Gaussian fit to the histogram. For a single simulation, we obtain $\alpha=-0.02 \pm 0.02$, while the average over the ensemble of gives $\langle\alpha\rangle=-0.045 \pm 0.010$. If the CMB intrinsic temperature anisotropies are not

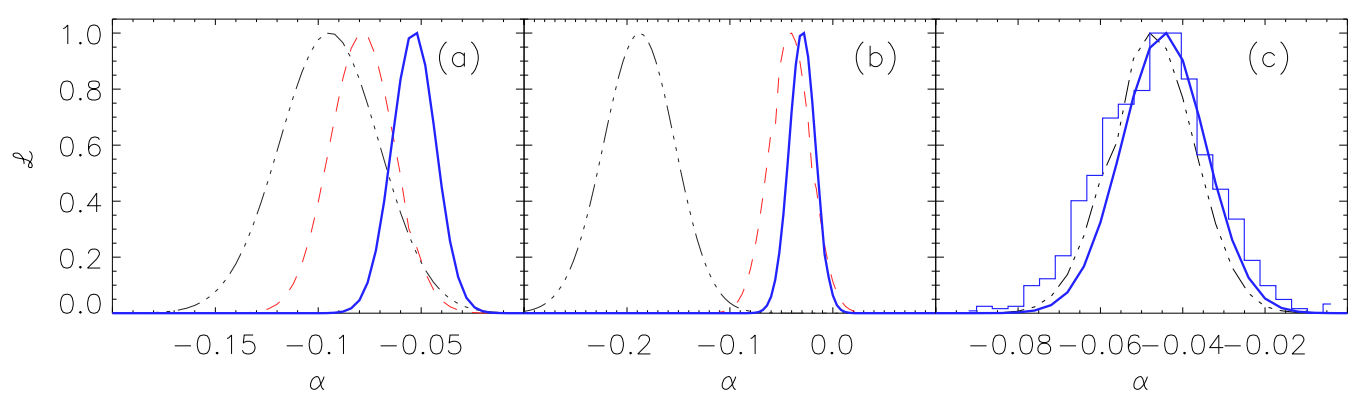

Figure 5.9: Likelihood function of the ratio method. (a) Likelihood for subsets of clusters selected by redshift, $z=([<0.11],[0.11-0.17],[>0.17])$. black dotted dash, red thin dashed, blue thick solid lines to clusters within the redshift intervals given in Table 5.1. (b) Likelihood for 3 mass intervals $M_{500}=([<0.192],[0.192-0.365],[>0.36]) \times 10^{15} M_{\odot} / h,(\mathrm{c})$ Full likelihood, including all clusters and the 10 different map ratios.

reduced significantly, the ratio method provides bad estimates of $\alpha$. For instance, if as large as $\Delta T_{C M B r e s}=10 \mu \mathrm{K}$, then for an $\alpha=0$ template, the measured value is $\alpha=-0.25 \pm 0.05$ (see Fig. 5.10).

We also computed the value of $\alpha$ with pipeline $B$. Using the 5 differencing maps $\Delta T_{[\nu-217 G H z]}(\hat{n})$ we constructed 8 different ratios $R_{[-217 G H z]}\left(\nu_{1}, \nu_{2}, \alpha\right)$. The results are presented in Fig. 5.11; lines follow the same conventions as in Fig. 5.9. In Fig. 5.11c we plot the full likelihood, including all clusters and all ratios. For this particular simulation $\alpha=0.00 \pm 0.01$. To check whether the method was biased, we carried out one thousand noise simulations and found that the average value and rms dispersion was $\langle\alpha\rangle=-0.003$ and $\sigma_{\alpha}=0.011$, removing the bias. The results of both pipeline $A$, and $B$ are summarized in Table 5.1. As one could expect, the most massive and the 


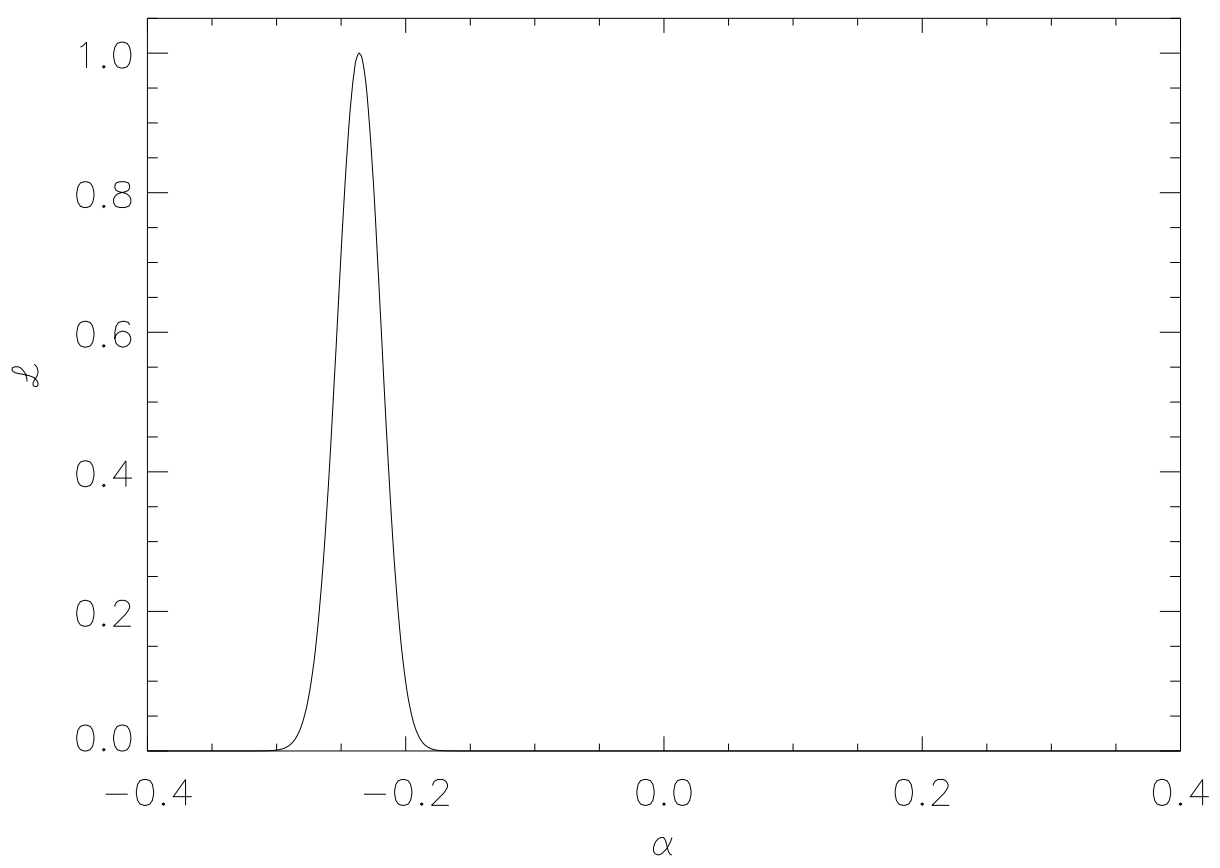

Figure 5.10: Likelihood computed including all clusters and all ratios for pipeline $A$ with residuals $\Delta T_{C M B \text { res }}=10 \mu \mathrm{K}$. While the input value is $\alpha=0$, the measured value is $\alpha=$ $-0.25 \pm 0.05$.

most distant clusters are the ones that provide the strongest constraint on $\alpha$.

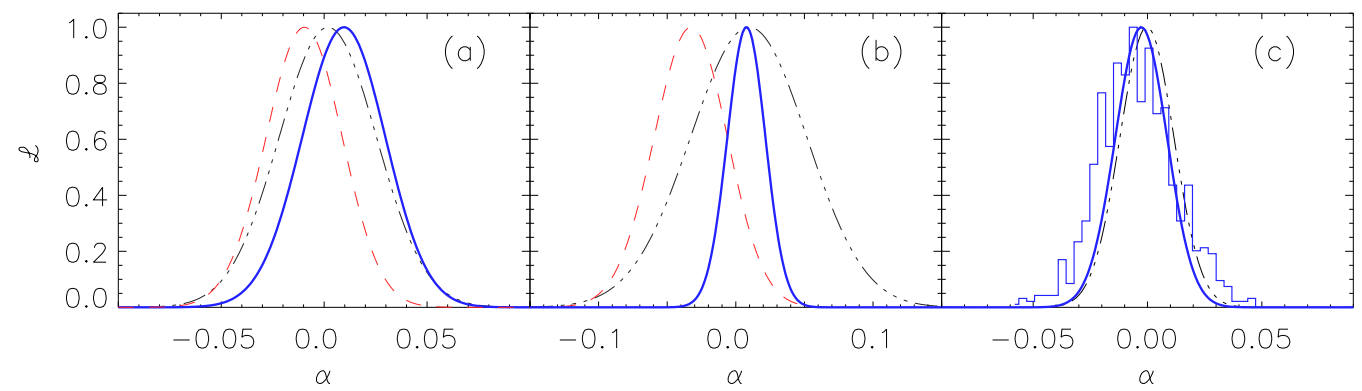

Figure 5.11: Likelihood function of the ratio method for pipeline B; panels and lines follow the conventions of Fig. 5.9.

To conclude, this method provides an unbiased estimate of $\alpha$ only when cosmological CMB signal and foreground residuals are removed to a level of few $\mu \mathrm{K}$. While we have not included foreground residuals in our analysis, we expect their effect not to be significant and we could infer their relative importance by analyzing cluster sub-samples selected according to Galactic latitude. 


\begin{tabular}{|c|ccc|}
\hline \hline & \multicolumn{3}{|c|}{ Redshift } \\
& $\leq \mathbf{0 . 1 1}$ & $\mathbf{0 . 1 1 - 0 . 1 7}$ & $>\mathbf{0 . 1 7}$ \\
\hline$\alpha_{A}$ & $0.10 \pm 0.02$ & $-0.08 \pm 0.02$ & $-0.05 \pm 0.01$ \\
$\alpha_{B}$ & $0.002 \pm 0.024$ & $0.01 \pm 0.02$ & $-0.01 \pm 0.02$ \\
\hline & \multicolumn{3}{|c|}{$M_{500} /\left[10^{15} M_{\odot} h^{-1}\right]$} \\
& $<\mathbf{0 . 1 9 2 8}$ & $\mathbf{0 . 1 9 2 8 - 0 . 3 6 3}$ & $>\mathbf{0 . 3 6 3}$ \\
\hline$\alpha_{A}$ & $-0.18 \pm 0.03$ & $-0.04 \pm 0.02$ & $-0.03 \pm 0.01$ \\
$\alpha_{B}$ & $0.01 \pm 0.04$ & $-0.03 \pm 0.03$ & $-0.008 \pm 0.014$ \\
\hline & & & \\
& $\mathbf{1} \mathbf{S k y}$ & $\mathbf{1 0 0 0} \mathbf{S k y}$ & \\
\hline$\alpha_{A}$ & $-0.02 \pm 0.02$ & $-0.045 \pm 0.010$ \\
$\alpha_{B}$ & $0.00 \pm 0.01$ & $-0.003 \pm 0.011$ \\
\hline \hline
\end{tabular}

Table 5.1: Results for a template of simulated clusters. Results from Fig. 5.9 and Fig. 5.11.

\subsubsection{Fit Method: analysis, systematics and results}

In this method we fit the frequency dependence of each cluster to the behavior in Figs. 5.2b (pipeline A) and 5.3b (pipeline B). The likelihood function is

$$
-2 \log \mathcal{L}=\sum_{\nu} \sum_{i=1}^{N_{c l}}\left[\frac{\left\langle\Delta T_{I}(\nu)\right\rangle-\bar{y}_{c} g_{I}(\nu, \alpha)}{\sigma_{\text {noise }, \nu, i}^{I}}\right]^{2},
$$

where $\sigma_{\text {noise }, \nu, i}^{I}=\sigma_{\text {noise }, \nu}^{I} / \sqrt{N_{\text {pix }, i}}$.

As indicated in Table 1.3, each frequency channel has different angular resolution. Convolution with the beam dilutes TSZ anisotropies differently at each frequency and the TSZ signal does not scale exactly as $g(\nu, 0)$. This effect is illustrated in Fig 5.12. Open squares represent the anisotropy measured by averaging the temperature difference $\Delta T_{[\nu,-217 G H z]}$ on a disc of radius $2 \theta_{500}$. The solid red lines represent the frequency dependence $g_{[-217 G H z]}(\nu, 0)$ given in Fig 5.3b. The dilution depends on the beam and the angular extent of the cluster. Fig 5.12a corresponds to a cluster at redshift $z=0.218$, with mass $M_{500}=3.64 \times 10^{14} \mathrm{M}_{\odot} \mathrm{h}^{-1}$ and angular size $9.4^{\prime}$, where as Fig $5.12 \mathrm{~b}$ to a cluster is at $z=0.058$, with mass $M_{500}=7.7 \times 10^{14} \mathrm{M}_{\odot} \mathrm{h}^{-1}$ and size $42^{\prime}$. The effect is largest at $44 \mathrm{GHz}$, the channel with the smallest resolution (see Table 1.3) and is more noticeable when the clusters are less extended. Therefore, to deconvolve the beam from the data, we will need a fit to the pressure profile of our clusters and for that, we need an independent measurement of the Comptonization parameter, $y_{c}$. Consequently, this method requires more information than the ratio method.

Hereafter, we will refer as deconvolution problem the problem of deconvolving the antenna beam from the measurement. In the catalog of simulated clusters the size, ellipticity and profile of each cluster so for such clusters, we could determine exactly 


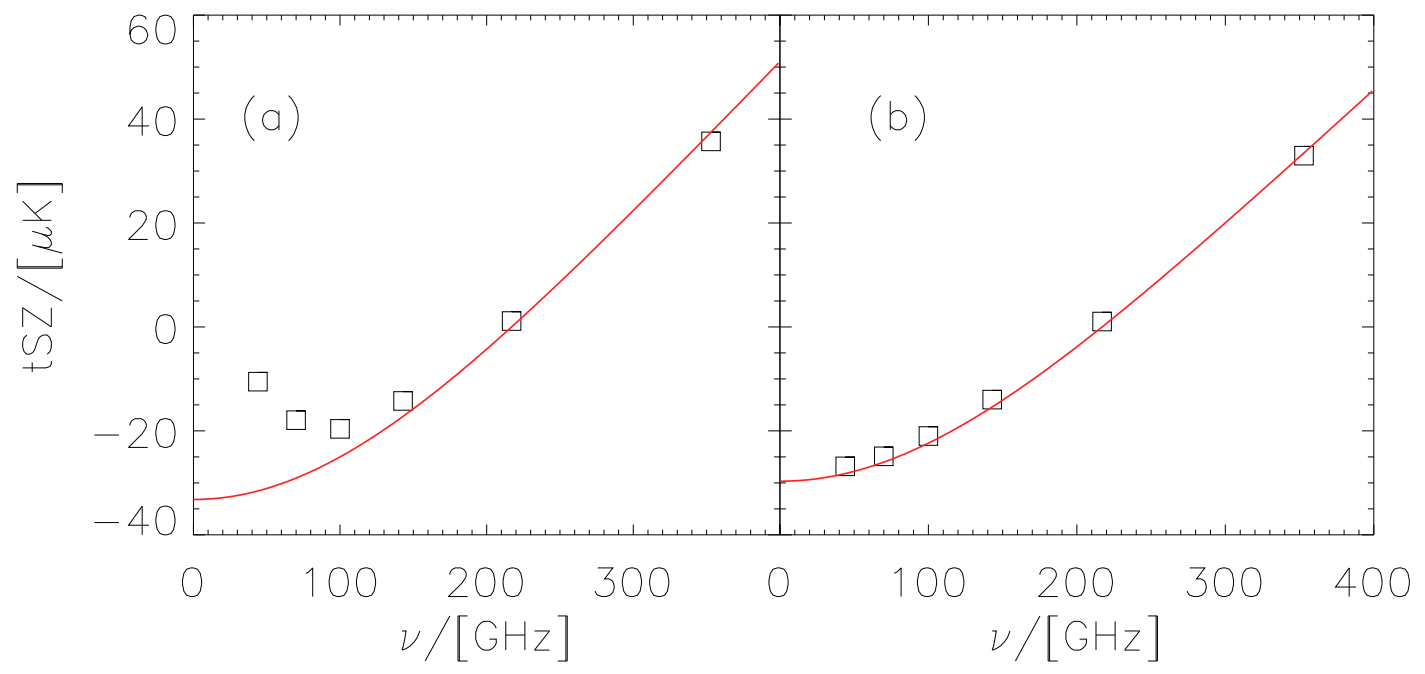

Figure 5.12: Effect of the beam dilution on the spectral dependence of the TSZ effect. Open squares correspond to the anisotropy measured on a disk of extent $2 \theta_{500}$. The solid line represents the TSZ scaling $g(\nu, 0)$. The data in (a) and (b) corresponds to clusters with masses $M_{500}=(3.64,7.7) \times 10^{14} \mathrm{~h}^{-1} \mathrm{M}_{\odot}$, are located at redshift $z=(0.218,0.058)$ and subtend an angle $\theta_{500}=\left(9.4^{\prime}, 42^{\prime}\right)$, respectively.

the deconvolution factor, $\mathcal{F}$, as

$$
\mathcal{F}=\frac{\left\langle y_{c}\right\rangle}{\left\langle y_{c} * B(\nu)\right\rangle},
$$

where $\left\langle y_{c}\right\rangle$ is the original Comptonization of the cluster and $\left\langle y_{c} * B(\nu)\right\rangle$ its convolution with the beam, averaged over a fixed solid angle. The factor $\mathcal{F}$ would be different for resolved and unresolved clusters, and it would depend on the cluster profile and redshift.

While $\mathcal{F}$ can be exactly measured for clusters extracted from a simulation, this is not the case for real clusters. To compute $\mathcal{F}$ we need to construct a pressure profile for each cluster. For our catalog of X-ray selected clusters, the only available information is restricted to the (smaller) X-ray emitting region. Then, we need to adopt several models for the pressure profile of the more extended TSZ temperature anisotropies. This will introduce an extra uncertainty when comparing the measured TSZ effect with the theoretical prediction. For illustration, in Fig 5.13 we plot the deconvolution factor as function of redshift for the Planck channels with the lowest and highest resolution: $44 \mathrm{GHz}$ (open squares) and $345 \mathrm{GHz}$ (solid black circles). Each panel corresponds to a different cluster subsample, selected by mass: (a) $M_{500}=(5-6) \times 10^{14} \mathrm{~h}^{-1} \mathrm{M}_{\odot}$ with a total of 110 clusters and (b) $M_{500}>6 \times 10^{14} \mathrm{~h}^{-1} \mathrm{M}_{\odot}$, with 131 clusters. To avoid overcrowding in (a) we plot only the clusters resolved at $345 \mathrm{GHz}$ and the unresolved ones at $44 \mathrm{GHz}$. In (b) all clusters are resolved at $353 \mathrm{GHz}$. At $44 \mathrm{GHz}$, clusters with redshift $z \geq 0.08$ are unresolved and only those are shown. Arrows indicate the deconvolution factor of the clusters plotted in Fig 5.12a,b. Finally, the 
straight solid lines represent a linear regression fit to the deconvolution factors in each mass range and channel. Let us denote the deconvolution factor estimated by linear
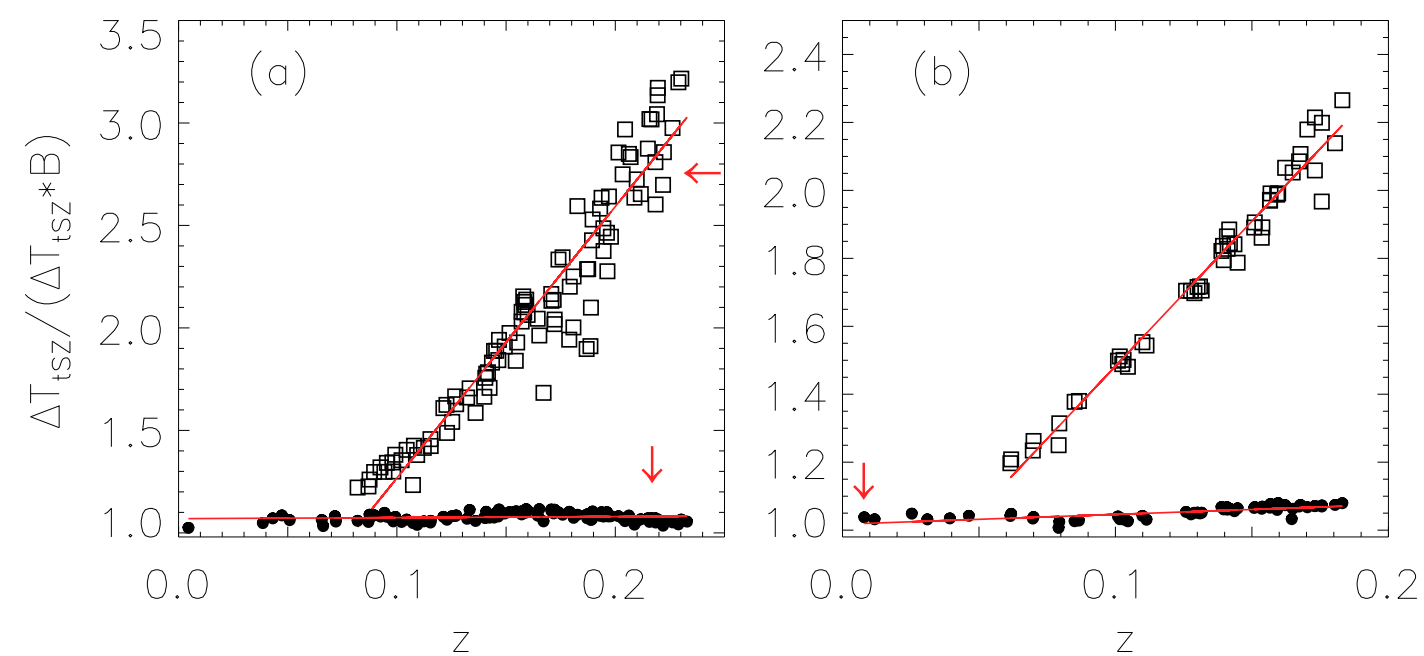

Figure 5.13: Deconvolution factor for clusters in the mass range (a) $M_{500}=5-6 \times 10^{14} \mathrm{~h}^{-1} \mathrm{M}_{\odot}$ and (b) $M_{500}>6 \times 10^{14} \mathrm{~h}^{-1} \mathrm{M}_{\odot}$. Solid black circles represent the deconvolution factor for the $353 \mathrm{GHz}$ channel and open squares for the $44 \mathrm{GHz}$ channel. All clusters are resolved at 353 $\mathrm{GHz}$ but, for simplicity, at $44 \mathrm{GHz}$ only the fraction of unresolved clusters is shown. Arrows indicate the deconvolution factor of the clusters of Fig. 5.12.

regression as $\mathcal{F}_{\text {lin }}$ and the dispersion around $\mathcal{F}_{\text {lin }}$ as $\Delta \mathcal{F}$. For our real clusters we will estimate their deconvolution using these relations, i.e., the deconvolution factor of eq. (5.12) will be

$$
\mathcal{F}=\mathcal{F}_{\text {lin }} \pm \Delta \mathcal{F}
$$

where $\Delta \mathcal{F}$ represents the uncertainty of the estimated deconvolution factor. The deconvolved temperature anisotropy will be

$$
\Delta T_{T S Z}^{d e c}=\left(y_{c} g(\nu, \alpha=0) * B\right) \mathcal{F}_{l i n},
$$

and it would differ from the the true signal $y_{c} g(\nu, \alpha=0)$ by an amount

$$
\sigma_{\mathcal{F}, i}=\left(y_{c} g(\nu, \alpha=0) * B\right) \Delta \mathcal{F} \text {. }
$$

As this uncertainty is uncorrelated with the instrumental noise at the cluster location, it can be included in the likelihood analysis of eq. 5.11 by adding it in quadrature to the instrumental noise

$$
\sigma_{\text {tot }, i}^{2}=\sigma_{\text {noise }, i}^{2}+\sigma_{\mathcal{F}, i}^{2} .
$$

In our pipeline we estimated deconvolution factors and their uncertainties in three mass bins of equal number of clusters. The bins chosen were the same that those used in the ratio method. The values for resolved and unresolved clusters and their uncertainties are given in Table 5.2. When a zero value is given, all clusters in that bin were resolved. 


\begin{tabular}{|c|c|ccc|}
\hline \hline & $\nu$ & \multicolumn{3}{|c|}{$\mathrm{M}_{500} /\left(\frac{10^{14} M_{\odot}}{h}\right)$} \\
\hline & & $<1.92$ & $1.92-3.65$ & $>3.65$ \\
\hline & & \multicolumn{3}{|c|}{ Resolved Clusters } \\
\hline & $44 \mathrm{GHz}$ & $0.187[187]$ & $0.078[45]$ & $0.053[26]$ \\
& $70 \mathrm{GHz}$ & $0.066[210]$ & $0.072[181]$ & $0.057[102]$ \\
$\Delta \mathcal{F}_{\text {lin }}$ & $100 \mathrm{GHz}$ & $0.054[210]$ & $0.037[210]$ & $0.043[210]$ \\
& $143 \mathrm{GHz}$ & $0.053[210]$ & $0.031[210]$ & $0.028[210]$ \\
& $353 \mathrm{GHz}$ & $0.054[210]$ & $0.030[210]$ & $0.024[210]$ \\
\hline & & \multicolumn{3}{|c|}{ Unresolved Clusters } \\
\hline & $44 \mathrm{GHz}$ & $0.143[23]$ & $0.247[165]$ & $0.183[184]$ \\
& $70 \mathrm{GHz}$ & $0.000[0]$ & $0.050[29]$ & $0.060[108]$ \\
$\Delta \mathcal{F}_{\text {lin }}$ & $100 \mathrm{GHz}$ & $0.000[0]$ & $0.000[0]$ & $0.000[0]$ \\
& $143 \mathrm{GHz}$ & $0.000[0]$ & $0.000[0]$ & $0.000[0]$ \\
& $353 \mathrm{GHz}$ & $0.000[0]$ & $0.000[0]$ & $0.000[0]$ \\
\hline $\mathrm{Ncl}$ & & 210 & 210 & 210 \\
\hline \hline
\end{tabular}

Table 5.2: Deconvolution Factors and their uncertainties for different frequencies and different subsamples selected according to their mass.

\section{Results for a template of simulated clusters}

We repeat the same set of simulations that in the ratio method, but considering only the case in which $\Delta T_{C M B r e s}=1 \mu \mathrm{K}$.

The results of Fig. 5.14 correspond to pipeline $A$; cluster subsamples and line conventions are those of Fig. 5.9: the black dot-dashed, red thin dashed and blue thick solid lines correspond to the low, intermediate high redshift/mass bins, respectively. Fig. 5.14c demonstrates that the fit method produces less bias and a more accurate determination of $\alpha$. Choosing one realization at random we found $\alpha=-0.02 \pm 0.03$, whereas the mean and dispersion from 1,000 realizations was $\langle\alpha\rangle=-0.02 \pm 0.02$.

In Fig. 5.15 we present the results for pipeline B. For one realization chosen at random we obtained $\alpha=-0.008 \pm 0.022$. This result is compatible with the input model, $\alpha=0$ at the $1 \sigma$ confidence level. The mean and rms of 1,000 simulations is $\langle\alpha\rangle=-0.017 \pm 0.021$.

To conclude, our numerical simulations clearly shows that the fit method is more accurate than the ratio method. It provides an unbiased estimate of $\alpha$. Therefore, if the Comptonization parameter $y_{c}$ can be derived from X-ray observations, the fit method is clearly superior to the ratio method. For reference, the results are summarized in Table 5.3. The table shows that for the randomly chosen simulations, pipeline $A$ is better than pipeline $B$ since it provides smaller error bars. But on the average over 


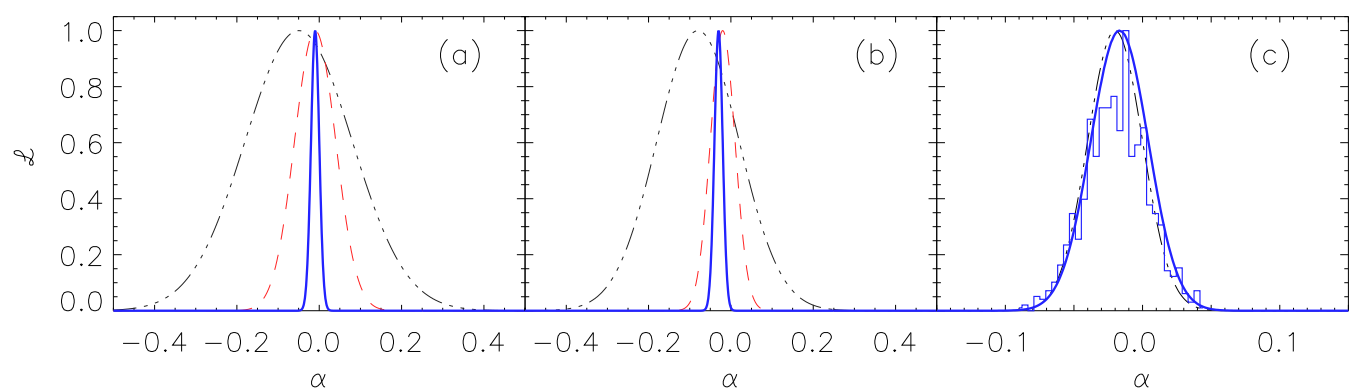

Figure 5.14: Likelihood function for different cluster subsets in pipeline A. Redshift intervals and line conventions are the same as in Fig. 5.11. In (a) clusters were selected by redshift; in (b) by mass. In (c) the black dash-dotted line represents the likelihood including all clusters and all frequencies. The blue solid line is the average likelihood of $10^{3}$ realizations of CMB residuals.

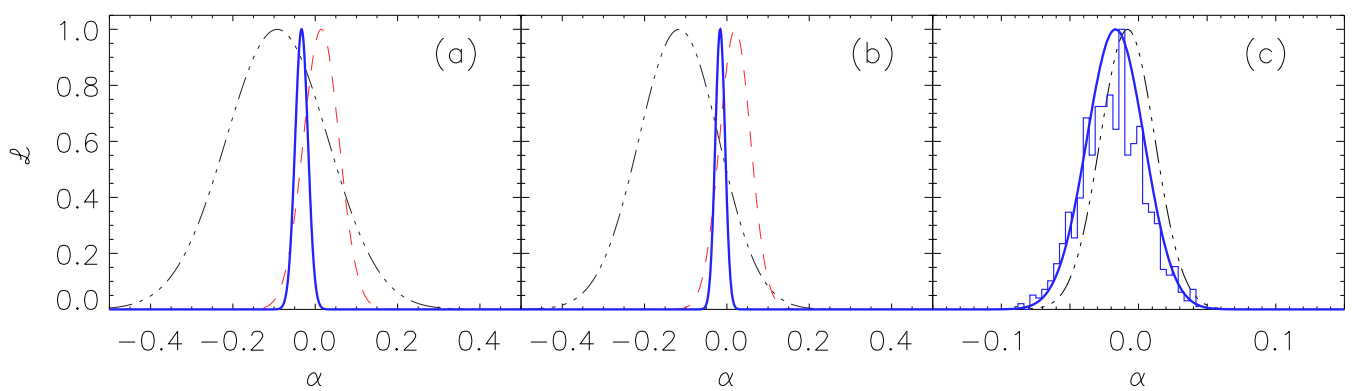

Figure 5.15: Likelihood function with the fit method in pipeline B. Lines follow the same convention as in Fig. 5.14.

1,000 simulations the errors are identical, indicating that both methods are equivalent.

\section{Results for a template of X-ray selected clusters}

We now proceed to estimate $\alpha$ using a catalog of real clusters. Since, as we have shown in the previous section, pipeline $A$ and $B$ give comparable results, we will only quote those of pipeline $B$. We constructed cluster pressure profiles using (1) the $\beta(=2 / 3)$-profile and (2) the universal pressure profile of eq.(4.23), with the parameters given in Table 4.2. At each frequency we deconvolve the antenna using the deconvolution factors of Table 5.2. We measured the temperature at each cluster location, fit the theoretically expected $g_{[-217 G H z]}(\nu, \alpha)$ of Fig. $5.3 \mathrm{~b}$ and computed the likelihoods for different cluster subsets. These likelihoods are presented in Fig. 5.16. Panels (a,b,c) correspond to the $\beta$-profile and panels (d,e,f) to the universal profile. Our results are summarized in Tables 5.4 and 5.5.

In Figs. 5.16(a) and (d) we represent the likelihood function for three different frequencies: $44 \mathrm{GHz}$ (dashed), $100 \mathrm{GHz}$ (solid) and $343 \mathrm{GHz}$ (dot-dashed line). The most restrictive result is from the $100 \mathrm{GHz}$ channel. The final likelihood is dominated by the channels that have the highest resolution and lowest noise. In Fig. 5.16(b) and 


\begin{tabular}{|c|ccc|}
\hline \hline & \multicolumn{3}{|c|}{ Redshift range } \\
& $<\mathbf{0 . 0 6 7 2}$ & $\mathbf{0 . 0 6 7 2 - 0 . 1 2 5}$ & $\mathbf{0 . 1 2 5 - 0 . 3 0 0}$ \\
\hline$\alpha_{A}$ & $-0.05 \pm 0.18$ & $-0.01 \pm 0.05$ & $-0.01 \pm 0.01$ \\
$\alpha_{B}$ & $-0.10 \pm 0.21$ & $0.02 \pm 0.07$ & $-0.02 \pm 0.03$ \\
\hline & \multicolumn{3}{|c|}{$\mathrm{M}_{500} /\left(\frac{10^{15} M_{\odot}}{h}\right)$} \\
& $<\mathbf{0 . 1 9 2}$ & $\mathbf{0 . 1 9 2 - 0 . 3 6 5}$ & $>\mathbf{0 . 3 6 5}$ \\
\hline$\alpha_{A}$ & $-0.08 \pm 0.13$ & $-0.02 \pm 0.03$ & $-0.03 \pm 0.02$ \\
$\alpha_{B}$ & $-0.12 \pm 0.11$ & $0.02 \pm 0.06$ & $-0.02 \pm 0.02$ \\
\hline & \multicolumn{3}{|c}{ Full sample } \\
& $\mathbf{1 ~ S k y}$ & $\mathbf{1 0 0 0} \mathbf{~ S k y}$ \\
\hline$\alpha_{A}$ & $-0.02 \pm 0.03$ & $-0.02 \pm 0.02$ \\
$\alpha_{B}$ & $-0.008 \pm 0.022$ & $-0.02 \pm 0.02$ \\
\hline \hline
\end{tabular}

Table 5.3: Results pipelines (A) and (B) on clusters selected from a N-body simulation. The figures correspond to the two randomly chosen realizations of Figs. 5.14 and 5.15, and to the average of 1,000 simulations.

\begin{tabular}{|cc|ccc||c|}
\hline \hline \multicolumn{5}{|c}{} & \multicolumn{4}{c|}{$\mathrm{M}_{500} /\left(\frac{10^{15} M_{\odot}}{h}\right)$} & \\
& $\nu(\mathrm{GHz})$ & $<\mathbf{0 . 1 9 2}$ & $\mathbf{0 . 1 9 2 - 0 . 3 6 5}$ & $>\mathbf{0 . 3 6 5}$ & $\mathbf{0 . 1 9 2}$ \\
\hline \hline $\mathrm{Ncl}$ & & 200 & 215 & 208 & 623 \\
\hline & $\mathbf{4 4}$ & $0.05 \pm 0.20$ & $-0.07 \pm 0.09$ & $0.025 \pm 0.026$ & $0.02 \pm 0.02$ \\
& $\mathbf{7 0}$ & $-0.05 \pm 0.21$ & $-0.04 \pm 0.8$ & $-0.007 \pm 0.024$ & $-0.01 \pm 0.02$ \\
$\alpha_{\nu}$ & $\mathbf{1 0 0}$ & $0.06 \pm 0.07$ & $-0.02 \pm 0.03$ & $0.003 \pm 0.007$ & $0.002 \pm 0.007$ \\
& $\mathbf{1 4 3}$ & $-0.04 \pm 0.11$ & $0.04 \pm 0.04$ & $0.02 \pm 0.01$ & $0.03 \pm 0.01$ \\
& $\mathbf{3 5 3}$ & $0.10 \pm 0.24$ & $0.005 \pm 0.093$ & $0.02 \pm 0.02$ & $0.02 \pm 0.02$ \\
\hline$\alpha$ & & $0.03 \pm 0.05$ & $-0.003 \pm 0.021$ & $0.011 \pm 0.006$ & $0.011 \pm 0.005$ \\
\hline & & & $\mathbf{D e c o n v o l u t i o n}$ & & \\
\hline & $\mathbf{4 4}$ & $-0.25 \pm 0.29$ & $-0.27 \pm 0.13$ & $-0.12 \pm 0.08$ & $-0.17 \pm 0.07$ \\
& $\mathbf{7 0}$ & $-0.10 \pm 0.20$ & $-0.12 \pm 0.10$ & $-0.03 \pm 0.03$ & $-0.04 \pm 0.03$ \\
$\alpha_{\nu}$ & $\mathbf{1 0 0}$ & $-0.01 \pm 0.10$ & $-0.03 \pm 0.03$ & $0.00 \pm 0.01$ & $-0.005 \pm 0.014$ \\
& $\mathbf{1 4 3}$ & $-0.06 \pm 0.13$ & $0.03 \pm 0.04$ & $0.02 \pm 0.01$ & $0.02 \pm 0.01$ \\
& $\mathbf{3 5 3}$ & $-0.008 \pm 0.265$ & $-0.008 \pm 0.096$ & $0.01 \pm 0.03$ & $0.01 \pm 0.03$ \\
\hline$\alpha$ & & $-0.05 \pm 0.07$ & $-0.02 \pm 0.02$ & $0.006 \pm 0.009$ & $0.002 \pm 0.009$ \\
\hline$\langle\alpha\rangle$ & & & - & - & $0.009 \pm 0.008$ \\
\hline \hline
\end{tabular}

Table 5.4: Constraints on $\alpha$ obtained using the $\beta(=2 / 3)$-profile. There are reported constraints from different subsamples, for frequency channel, and the total ones.

(e), we represent the likelihood for the three mass bins used in the ratio analysis. We have marginalized over frequencies. Dashed, dot-dashed and solid lines correspond to the low, intermediate and high mass intervals. The most massive clusters dominate the signal since they have stronger signal and are, on average, at higher redshift than the lower and intermediate mass samples. Fig. 5.16(c) and (f) represent 1 realization of the 


\begin{tabular}{|c|c|c|c|c|c|}
\hline & $\nu(\mathrm{GHz})$ & $<0.192$ & $\begin{array}{r}M_{500} \\
\mathbf{0 . 1 9 2 - 0 . 3 6 5}\end{array}$ & $\begin{array}{l}\left.\frac{10^{15} M_{\odot}}{h}\right) \\
>\mathbf{0 . 3 6 5}\end{array}$ & $>0.192$ \\
\hline $\mathrm{Ncl}$ & & 200 & 215 & 208 & 623 \\
\hline & 44 & $>1.0$ & $<-1.0$ & $<-1.0$ & $<-1.0$ \\
\hline & 70 & $>1.0$ & $0.60 \pm 1.92$ & $-0.30 \pm 0.50$ & $0.31 \pm 0.46$ \\
\hline$\alpha_{\nu}$ & 100 & $-0.21 \pm 0.62$ & $0.03 \pm 0.23$ & $0.005 \pm 0.071$ & $0.005 \pm 0.068$ \\
\hline & 143 & $0.49 \pm 0.27$ & $0.11 \pm 0.11$ & $0.002 \pm 0.031$ & $0.01 \pm 0.03$ \\
\hline & 353 & $-0.67 p m 0.40$ & $0.03 \pm 0.14$ & $-0.02 \pm 0.03$ & $-0.02 \pm 0.03$ \\
\hline$\alpha$ & & $0.06 \pm 0.16$ & $0.02 \pm 0.06$ & $0.07 \pm 0.014$ & $0.007 \pm 0.014$ \\
\hline & & \multicolumn{3}{|c|}{ Deconvolution } & \\
\hline \multirow{5}{*}{$\alpha_{\nu}$} & 44 & $-0.40 \pm 0.90$ & $-0.40 \pm 0.40$ & $-0.28 \pm 0.14$ & $-0.30 \pm 0.13$ \\
\hline & 70 & $0.05 \pm 0.82$ & $-0.40 \pm 0.30$ & $-0.06 \pm 0.08$ & $-0.07 \pm 0.07$ \\
\hline & 100 & $0.23 \pm 0.30$ & $-0.14 \pm 0.11$ & $0.002 \pm 0.027$ & $-0.004 \pm 0.026$ \\
\hline & 143 & $-0.18 \pm 0.42$ & $0.20 \pm 0.15$ & $0.06 \pm 0.03$ & $0.06 \pm 0.03$ \\
\hline & 353 & $0.60 \pm 0.84$ & $0.12 \pm 0.30$ & $0.04 \pm 0.06$ & $0.05 \pm 0.06$ \\
\hline$\alpha$ & & $0.10 \pm 0.22$ & $-0.06 \pm 0.08$ & $0.020 \pm 0.018$ & $0.020 \pm 0.020$ \\
\hline$\langle\alpha\rangle$ & & - & - & - & $-0.011 \pm 0.018$ \\
\hline
\end{tabular}

Table 5.5: Constraints on $\alpha$ obtained using the universal profile. There are reported constraints from different subsamples, for frequency channel, and the total ones.

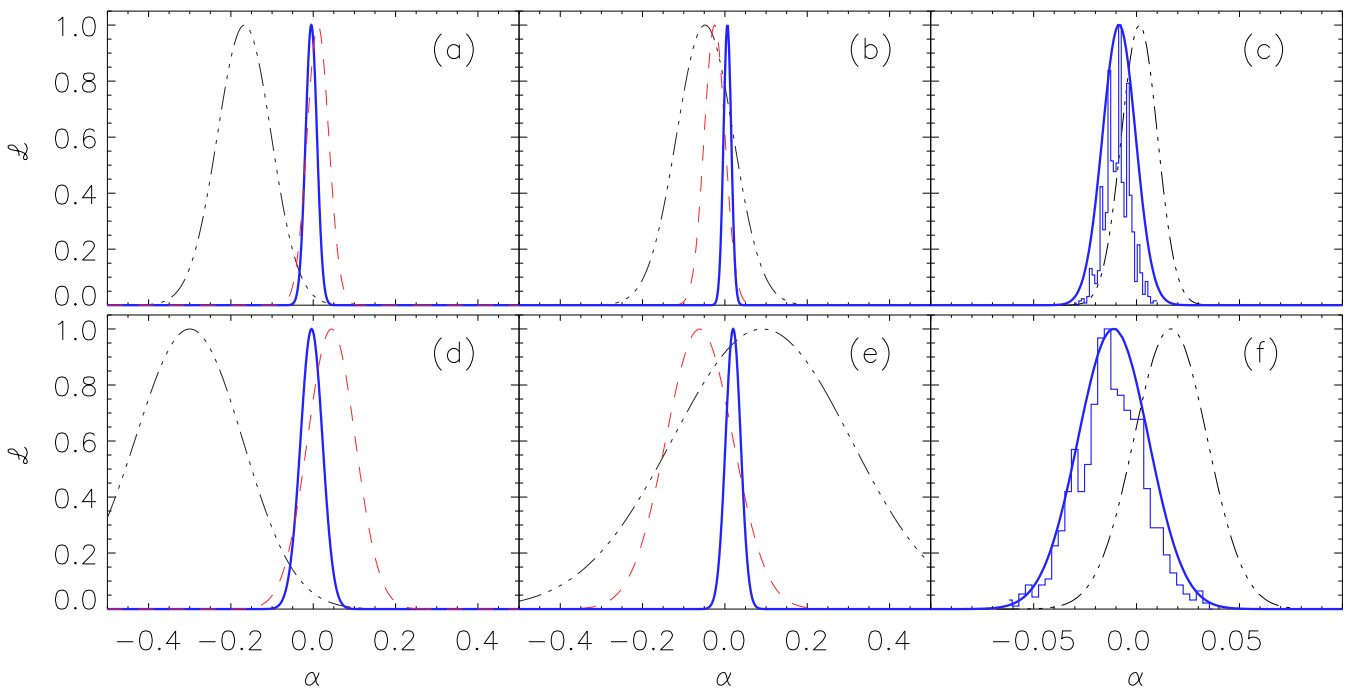

Figure 5.16: Likelihood functions for the fit method using our pipeline (B) applied to a catalog of real clusters. In panels ( $\mathrm{a}, \mathrm{b}, \mathrm{c}$ ) cluster profiles were $\beta=2 / 3$ models with the values of Table 5.4; in (d,e,f) clusters were the universal profiles of Table 5.5. In (a,d), (e,f) the black dotted dash, red thin dashed, blue thick solid lines corresponds to the 44, 100, and $143 \mathrm{GHz}$ channel and to intermediate and high mass bin, respectively. In (c,f) the black dash-dotted line corresponds to the full likelihood of a single realization, including all clusters and all frequencies, and the blue solid line is the average likelihood of $10^{3}$ realizations.

sky (dot-dashed line), and the histograms of 1,000 simulations constructed using the $\beta=2 / 3$ and the universal pressure profile. Blue solid lines are a fit to the histogram. The mean and rms dispersion of the estimated values are $\langle\alpha\rangle=-0.011 \pm 0.018$ for 
the universal profile and $\langle\alpha\rangle=0.009 \pm 0.008$ for the $\beta$-model profile. Since all cluster properties are identical, then TSZ effect integrated over the cluster extent will be larger for the $\beta$-model than for the universal profile, as shown in Fig 5.6a. Therefore, it must constrain $\alpha$ better, as shown.

As a conclusion, our results show that a catalog of real, X-ray selected clusters constrain $\alpha$ as well as clusters drawn from an hydrodynamical N-Body simulation. Simulated clusters include all projection effects up to redshift $z=0.25$. These effects are not included in the templates of our real clusters, and being the results comparable, projection effects can not play a significant role. Indeed, as shown in Fig 5.16e, the full likelihood is dominated by the most massive clusters for which projection effects have been shown not to be significant.

\subsection{Discussion and future perspectives}

In this Chapter we have analyzed the possibility of constraining the evolution history of the CMB blackbody temperature using Planck data. This evolution is usually constrained using excitation lines in quasars at redshifts $z \simeq 2-3$. We have explored if the TSZ effect can offer similar o better precision to the more interesting redshift range $z \leq 1$, when the Universe expansion started to accelerate. We have tested two different, but complementary, methodologies: (A) Taking ratios of temperatures at two different frequencies provides biased estimates, dominated by the errors in the denominator; (B) fits of the spectral dependence of the TSZ effect provides an unbiased estimation but requires to determine the mean Comptonization parameter by independent means. The bias in method (A) can be reduced by choosing low noise data in the denominator. The data needed in (B) could be derived from X-ray measurements. Comparison of results at different frequencies involves deconvolving the data from the antenna beam. The deconvolution could affect the constraints derived from frequency channels with different angular resolution and deconvolution requires the cluster profile to be known. Since the error introduced by the deconvolution is uncorrelated with noise and foreground residuals, it can be easily included in the error bar as indicated by eq. (5.16).

We predict that the final uncertainty on $\alpha$ will be of the order on $0.01-0.02$ that represents an improvement of a factor $2-3$ better than those obtained from quasar spectra by Noterdaeme et al. (2011). Let us remark that the rms dispersion of $\alpha$ on 1,000 simulations, $\sigma_{\alpha}$, is very similar to the error on $\alpha$ in one single realization, both in the ratio and in the fit method, indicating that our pipelines are efficient. However, the final accuracy will depend on how well foregrounds can be removed from the data.

At present our catalog is restricted to clusters with $z \leq 0.3$, we have not extended our analysis beyond that redshift. Adding more clusters with current or future obser- 
vations will help to detect possible deviations from adiabatic evolution, especially if clusters have higher mass and are at higher redshifts. A extended dataset will improve the precision on $\alpha$ beyond the 1-2\% level.

In April 2013 Planck released its first nominal maps after one year of integration. For the purposes of this investigation the Planck collaboration did not provide provide foreground clean maps, or the y-Compton map. At present, we are developing techniques to clean up the data using Planck ancillary information. In the meanwhile, Hurier et al. (2014) have constrained $\alpha=0.009 \pm 0.017$ applying the methodology discussed in this chapter. Since they were part of the Planck Collaboration, they could use the MILCA code ${ }^{1}$ to produce the Planck $y$-Compton map and use it to constrain the CMB blackbody temperature evolution. We are trying to carry out an independent analysis based on the techniques elaborated here, but so far we have found that foreground residuals are an important effect.

\footnotetext{
${ }^{1}$ MILCA: modified internal linear component algorithm (MILCA) that generalizes the ILC approach to the case of multiple astrophysical components for which the electromagnetic spectrum is known (Hurier et al., 2013).
} 


\title{
Conclusions and future perspectives.
}

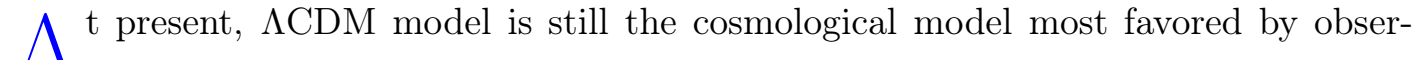
1 vations (Sect. 1.3). It presents several theoretical difficulties that need to be understood in order to reach a self-consistent description of the Universe at every scale. These shortcomings could be overcome by extending the theory of gravitational interaction from GR to more general theories, termed generically as ETGs. In these theories, dark matter and dark energy are not needed to explain the present period of accelerated expansion of the Universe or the dynamics of galaxies. Nevertheless, a definitive alternative theory of gravity has not been reached. ETGs give rise to fourth order partial differential field equations that are more complicated to solve and the results more difficult to compare with observations. Conceptually, there agreement has not been reached on the best way to formulate an alternative theory of gravity, and many controversies have not been solved yet (Sect. 1.6.1), ETGs have attracted interest in the last decades. There exists a methodological approach that allows to compare directly whatever version of ETGs with GR. The idea is that any relativistic theory of gravity has to recover the well-tested results of GR in its weak field limit. Many mechanisms have been proposed to guarantee that the tight constraints from observations are met (Sect. 1.6.3). In order to reach a definitive theory of gravita-
\end{abstract} tion, ETGs need to be tested in all possible astrophysical and cosmological scenarios and scales. In this thesis, we have focused our work in testing analytical $f(R)$ gravity models, a particular class of ETGs, that are naturally screened at Solar System scale without invoking any outer mechanisms but resorting to an extra scale length at its weak field limit. Our calculations were performed in the Jordan frame without conformal transformations to the Einstein frame since such transformations could obscured the interpretation of the results.

In order to test ETGs at Solar system scale, we analyzed the Jeans instability in $f(R)$ gravity in the Newtonian limit of the theory, following the classical procedure. We obtained a new dispersion relation eq. (2.42) that led to a new Jeans length (2.43). We argued that the Newtonian value is an upper limit for the Jean mass corresponding 
to $f(R)=R$ or, in other words, when the correction to the Lagrangian are negligible, $f_{0}^{\prime \prime} \simeq 0$. The approach can be generalized to other versions of alternative theories of gravity, and represent a first test for any of these theories. This work needs to be generalized by introducing magnetic field, collisions and turbulence in order to construct more realistic models of fragmentation phase of the interstellar clouds in order to describe the formation and evolution of the stars in $f(R)$ gravity.

Next, we tested the strong field regime at Solar System scales by analyzing the GWs emitted by the binary system. We found an analytical formula for the first derivative of the orbital period in $f(R)$ gravity (3.49) and we compared the predictions of both theories, GR and ETGs, using a sample of relativistic binary systems. Our results give a clear indication that the dynamic and the emission of GWs in binary systems can be accommodated in ETGs and, for some particular systems with very accurate mass estimates it is possible to obtain an upper limit on graviton mass which is consistent with results presented in literature. However, we need to further improve the calculations computing all Post-Keplerian parameters and masses using the ETG itself. The data also need to be improved; the masses need to be estimated without assumptions about the mass of the pulsar or the inclination of the orbit, taking into account the effect of mass transfer within the binary system.

We studied ETGs on cluster scales. We constructed the cluster pressure profile of gas in hydrostatic equilibrium in a potential well solution of $f(R)$-gravity. Our model depends on two $f(R)$ parameters and the equation of state of the gas, taken to be polytropic. The model was integrated along the line of sight in order to construct the cluster profile and compare it to the data. As data we used the SMICA map provided by Planck Collaboration in April 2013 and a proprietary X-ray cluster catalog. We stacked the cluster profile on the SMICA map and we compared the data with our theoretical models producing the contours of confidence levels on pairs of parameters. We showed that the Yukawa-like correction to the Newtonian potential can provide a viable alternative to explain the cluster pressure profile without DM. We expect to obtain tighter constraints by repeating the analysis in maps at different frequencies and fitting the cluster profile cluster by cluster. However, this requires foreground clean maps that are in preparation.

Finally, we explored how to test adiabatic evolution of the Universe using Planck CMB data. We considered two types of datasets depending on how the the cosmological signal was removed: using a filter or using the $217 \mathrm{GHz}$ map. We applied two different statistical estimators, based on the ratio of temperature anisotropies at two different frequencies, and on a fit to the spectral variation of the cluster signal with frequency. The ratio method is very sensitive to the CMB residuals present in the data and if they have an amplitude close to $\sim 10 \mu \mathrm{K}$ give strongly biased results. The fit method is not so sensitive to the presence of residuals. To test for systematics, we construct a template 
from clusters drawn from a hydro-simulation included in the pre-launch Planck Sky Model. We demonstrate that, using a proprietary catalog of X-ray selected clusters with measured redshifts, electron densities and X-ray temperatures, we can constrain deviations of adiabatic evolution, measured by the parameter $\alpha$ in the redshift scaling $T(z)=T_{0}(1+z)^{1-\alpha}$, with an accuracy of $\sigma_{\alpha}=0.011$ in the most optimal case and with $\sigma_{\alpha}=0.018$ for a less optimal case. These results represent a factor 2-3 improvement over similar measurements carried out using quasar spectral lines and a factor 6-20 with respect to earlier results using smaller cluster samples. We will applied the techniques developed here to foreground clean Planck maps once they become available. 


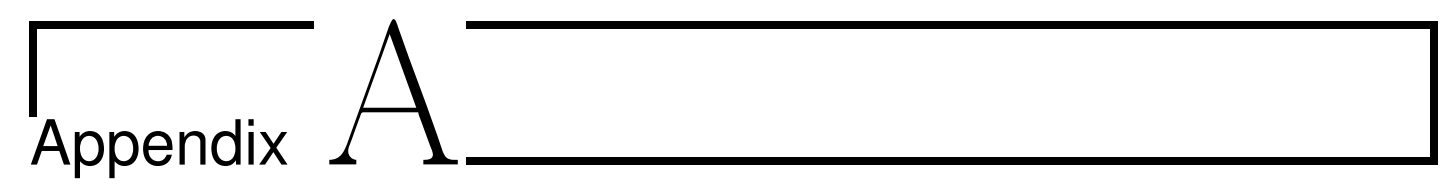

\section{Fundamental constants and conversion}

\section{factors}

The main purpose of this appendix is to collect together some of the physical constant, and unit conversions that are most used in this thesis.

\begin{tabular}{|lccc|}
\hline \hline Name & Symbol & Value & Unit \\
\hline Boltzmann constant & $k_{B}$ & $=1.3806488 \times 10^{-23}$ & $\mathrm{~J} \mathrm{~K}^{-1}$ \\
& & $=8.6173324 \times 10^{-5}$ & $\mathrm{eV} \mathrm{K}{ }^{-1}$ \\
Electron mass energy equivalent & $m_{e} c^{2}$ & $=0.510998928$ & $\mathrm{MeV}$ \\
Gravitation constant & $G$ & $=6.67428(67) \times 10^{-11}$ & $\mathrm{~kg}^{-1} \mathrm{~m}^{3} \mathrm{~s}^{-2}$ \\
Planck constant & $\hbar$ & $=1.054571 \times 10^{-34}$ & $\mathrm{~J} \mathrm{~s}$ \\
& & $=6.582843 \times 10^{-16}$ & $\mathrm{eV} \mathrm{s}$ \\
Thomson cross section & $\sigma_{T}$ & $0.6652458734 \times 10^{-28}$ & $\mathrm{~m}^{2}$ \\
Speed of light in vacuum & $c$ & $2.99792458 \times 10^{8}$ & $\mathrm{~m} \mathrm{~s}^{-1}$ \\
\hline \hline
\end{tabular}

Table A.1: Physical constants of interest. 


\begin{tabular}{|lc|}
\hline \hline Name & Conversion factor \\
\hline Astronomical Unit & $\mathrm{AU}=149597871 \times 10^{3} \mathrm{~m}$ \\
Dimensionless Hubble parameter & $h=H_{0} / 100 \mathrm{~km} \mathrm{~s}^{-1} \mathrm{Mpc}^{-1}$ \\
Energy & $1 \mathrm{GeV}=1.6022 \times 10^{-10} \mathrm{~J}$ \\
Hubble time & $H_{0}^{-1}=9.7776 \times 10^{9} \mathrm{~h}^{-1} \mathrm{yr}$ \\
Hubble radius & $c / H_{0}=2997.9 \mathrm{~h}^{-1} \mathrm{Mpc}$ \\
keV as temperature unit & $1 \mathrm{keV}=1.160510^{7} \mathrm{~K}$ \\
Parsec & $1 \mathrm{pc}=3.0856 \times 10^{16} \mathrm{~m}$ \\
Solar Luminosity & $1 \mathrm{~L}_{\odot}=3.90 \times 10^{26} \mathrm{~W}$ \\
Solar Mass & $1 \mathrm{M}_{\odot}=1.989 \times 10^{30} \mathrm{~kg}$ \\
\hline \hline
\end{tabular}

Table A.2: Astrophysical and Cosmological quantities of interest. 


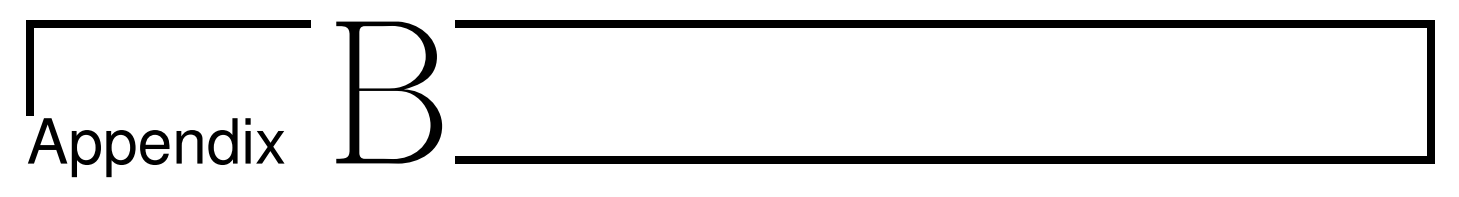

\section{Yukawa-like correction to Newtonian gravitational potential}

In this appendix we will show that in analytic $f(R)$-gravity the weak field limit gives rise to a Yukawa-like correction to Newtonian gravitational potential. We will use the metric approach, where the connections are function of the metric tensor.

To evaluate the Post-Newtonian limit, we need to consider corrections up to fourth order in the perturbation expansion of the metric. We start by expanding the metric in Taylor series of $v^{2}$ and we obtain (Weinberg, 1972)

$$
\begin{aligned}
& g_{00}(c t, r)=1+g_{00}^{(2)}(c t, r)+g_{00}^{(4)}(c t, r)+\mathcal{O}(6), \\
& g_{i j}(c t, r)=-\delta_{i j}+g_{i j}^{(2)}(c t, r)+g_{i j}^{(4)}(c t, r)+\mathcal{O}(6), \\
& g_{i 0}(c t, r)=g_{i 0}^{(3)}(c t, r)+g_{i 0}^{(5)}(c t, r)+\mathcal{O}(6)
\end{aligned}
$$

The inverse of the metric tensor verifies

$$
\begin{gathered}
g^{i \mu} g_{0 \mu}=g^{i 0} g_{00}+g^{i j} g_{0 j}=0 \\
g^{0 \mu} g_{0 \mu}=g^{00} g_{00}+g^{0 j} g_{0 j}=1 \\
g^{i \mu} g_{j \mu}=g^{i 0} g_{j 0}+g^{i k} g_{j k}=\delta_{i j} .
\end{gathered}
$$

Carrying out the Taylor expansion

$$
\begin{aligned}
& g^{00}(c t, r)=1+g^{00^{(2)}}(c t, r)+g^{00^{(4)}}(c t, r)+\ldots \\
& g^{i j}(c t, r)=-\delta^{i j}+g^{i j^{(2)}}(c t, r)+g^{i j^{(4)}}(c t, r)+\ldots \\
& g^{i 0}(c t, r)=g^{i 0^{(3)}}(c t, r)+g^{i 0^{(5)}}(c t, r)+\ldots
\end{aligned}
$$

we obtain

$$
\begin{array}{lll}
g^{00^{(2)}}=-g_{00}^{(2)} ; & g^{i j^{(2)}}=-g_{i j}^{(2)} ; & g^{i 0^{(3)}}=g_{i 0}^{(3)} \\
g^{00^{(4)}}=-g_{00}^{(4)} ; & g^{i j^{(4)}}=-g_{i j}^{(4)} ; & g^{i 0^{(5)}}=g_{i 0}^{(5)}
\end{array}
$$


To derive the Post-Newtonian limit of a single isolated mass we will consider spherically symmetric solutions with $\left(x^{0}, x^{1}, x^{2}, x^{3}\right) \equiv(c t, r, \theta, \varphi)$; then the metric can be written as

$$
d s^{2}=g_{\mu \nu} d x^{\mu} d x^{\nu}=g_{00}(c t, r) c^{2} d t^{2}-g_{11}(c t, r) d r^{2}+r^{2} d \Omega^{2},
$$

where $d \Omega^{2}$ is the solid angle. Eqs. (B.1)-(B.3) and (B.7)-(B.11) allow us to re-write the metric as follows

$$
\begin{aligned}
& g_{00}(c t, r)=1+g_{00}^{(2)}(c t, r)+g_{00}^{(4)}(c t, r), \\
& g_{11}(c t, r)=-1+g_{11}^{(2)}(c t, r) \\
& g_{22}(c t, r)=-r^{2} \\
& g_{33}(c t, r)=-r^{2} \sin \theta^{2},
\end{aligned}
$$

and

$$
\begin{aligned}
& g^{00}(c t, r)=1+\mathrm{g}^{00^{(2)}}(c t, r)+\mathrm{g}^{00^{(4)}}(c t, r)=1-g_{00}^{(2)}(c t, r)-g_{00}^{(4)}(c t, r), \\
& g^{11}(c t, r)=-1+\mathrm{g}^{11^{(2)}}(c t, r)=-1-g_{11}^{(2)}(c t, r), \\
& g^{22}(c t, r)=-r^{2}, \\
& g^{33}(c t, r)=-r^{2} \sin \theta^{2} .
\end{aligned}
$$

When evaluating the Christoffel symbols using eq. (1.4), we have to take into account that the space and time derivatives are of order

$$
\frac{\partial}{\partial x^{i}} \sim \frac{1}{r}, \quad \frac{\partial}{\partial x^{0}} \sim \frac{v}{r} .
$$

For the sake of simplicity, let us take $c=1$; then the Christoffel symbols at different orders are

For $\alpha=0$ :

$$
\Gamma_{\mu \nu}^{0}=\frac{1}{2} g^{0 \lambda}\left(g_{\lambda \mu, \nu}+g_{\nu \lambda, \mu}-g_{\mu \nu, \lambda}\right)=g^{00}\left(g_{0 \mu, \nu}+g_{\nu 0, \mu}-g_{\mu \nu, 0}\right),
$$

and the only non-vanishing components are

$$
\begin{aligned}
& \text { - } \Gamma_{11}^{0}=-\frac{1}{2}\left(1-\mathrm{g}_{00}^{(2)}-\mathrm{g}_{00}^{(4)}\right) \partial_{t} \mathrm{~g}_{11}^{(2)} \approx \frac{1}{2}\left(\partial_{t} \mathrm{~g}_{11}^{(2)}-\mathrm{g}_{00}^{(2)} \partial_{t} \mathrm{~g}_{11}^{(2)}\right)=\frac{1}{2} \partial_{t} \mathrm{~g}_{11}^{(2)}=\Gamma_{11}^{0} \\
& \text { - } \Gamma_{01}^{0}=\Gamma_{10}^{0}=\frac{1}{2}\left(1-\mathrm{g}_{00}^{(2)}-\mathrm{g}_{00}^{(4)}\right)\left(\partial_{r} \mathrm{~g}_{00}^{(2)}+\partial_{r} \mathrm{~g}_{00}^{(4)}\right) \approx \\
& \approx \frac{1}{2}\left(\partial_{r} \mathrm{~g}_{00}^{(2)}-\mathrm{g}_{00}^{(2)} \partial_{r} \mathrm{~g}_{00}^{(2)}+\partial_{r} \mathrm{~g}_{00}^{(4)}\right)=\Gamma_{01}^{0}+\Gamma_{01}^{0} \\
& \text { - } \Gamma_{00}^{0}=\frac{1}{2}\left(1-\mathrm{g}_{00}^{(2)}-\mathrm{g}_{00}^{(4)}\right)\left(\partial_{t} \mathrm{~g}_{00}^{(2)}+\partial_{t} \mathrm{~g}_{00}^{(4)}\right) \approx \\
& \approx \frac{1}{2}\left(\partial_{t} \mathrm{~g}_{00}^{(2)}-\mathrm{g}^{00(2)} \partial_{t} \mathrm{~g}_{00}^{(2)}+\partial_{t} \mathrm{~g}_{00}^{(4)}\right)=\frac{1}{2}\left(\partial_{t} \mathrm{~g}_{00}^{(2)}\right)=\stackrel{(3)}{0}
\end{aligned}
$$


For $\alpha=1$ :

$$
\Gamma_{\mu \nu}^{1}=\frac{1}{2} g^{1 \lambda}\left(g_{\lambda \mu, \nu}+g_{\nu \lambda, \mu}-g_{\mu \nu, \lambda}\right)=g^{11}\left(g_{1 \mu, \nu}+g_{\nu 1, \mu}-g_{\mu \nu, 1}\right)
$$

with the following non-vanishing components

$$
\begin{aligned}
& \text { - } \Gamma_{11}^{1}=\frac{1}{2}\left(-1-\mathrm{g}_{11}^{(2)}\right) \partial_{r} \mathrm{~g}_{11}^{(2)} \approx-\frac{1}{2}\left(\partial_{r} \mathrm{~g}_{11}^{(2)}+\mathrm{g}_{11}^{(2)} \partial_{r} \mathrm{~g}_{11}^{(2)}\right)=\Gamma_{11}^{1}+\Gamma_{11}^{1} \\
& \text { - } \Gamma_{22}^{1}=-\left(1+\mathrm{g}_{11}^{(2)}\right) r=\left(1+\mathrm{g}_{11}^{(2)}\right) \partial_{r} \mathrm{~g}_{22}^{(2)} \approx \Gamma_{22}^{(2)}+\Gamma_{22}^{1} ; \\
& \text { - } \Gamma_{33}^{1}=-\left(1+\mathrm{g}_{11}^{(2)}\right) r \sin ^{2} \theta=\left(1+\mathrm{g}_{11}^{(2)}\right) \partial_{r} \mathrm{~g}_{33}^{(2)} \approx \Gamma_{33}^{(2)}+\Gamma_{33}^{1} ; \\
& \text { - } \Gamma_{00}^{1}=-\frac{1}{2}\left(-1-\mathrm{g}_{11}^{(2)}\right)\left(\partial_{r} \mathrm{~g}_{00}^{(2)}+\partial_{r} \mathrm{~g}_{00}^{(4)}\right) \approx \\
& \approx \frac{1}{2}\left(\partial_{r} \mathrm{~g}_{00}^{(2)}+\mathrm{g}_{11}^{(2)} \partial_{r} \mathrm{~g}_{00}^{(2)}+\partial_{r} \mathrm{~g}_{00}^{(4)}\right)=\Gamma_{00}^{(2)}+\Gamma_{00}^{(4)} \\
& \text { - } \Gamma_{01}^{1}=\Gamma_{10}^{1}=-\frac{1}{2}\left(1+\mathrm{g}_{11}^{(2)}\right) \partial_{t} \mathrm{~g}_{11}^{(2)} \approx \\
& \approx-\frac{1}{2}\left(\partial_{t} \mathrm{~g}_{11}^{(2)}+\mathrm{g}^{11(2)} \partial_{t} \mathrm{~g}_{11}^{(2)}\right)=\frac{1}{2} \partial_{t} \mathrm{~g}_{11}^{(2)}=\Gamma_{01}^{1}
\end{aligned}
$$

For $\alpha=2$ :

$$
\Gamma_{\mu \nu}^{2}=\frac{1}{2} g^{2 \lambda}\left(g_{\lambda \mu, \nu}+g_{\nu \lambda, \mu}-g_{\mu \nu, \lambda}\right)=g^{22}\left(g_{2 \mu, \nu}+g_{\nu 2, \mu}-g_{\mu \nu, 2}\right)
$$

and the non-vanishing components are

$$
\begin{aligned}
& \text { - } \Gamma_{33}^{2}=-r^{4} \cos \theta \sin \theta=-\mathrm{g}_{22}^{(2)} \partial_{\theta} \mathrm{g}_{33}^{(2)}=\stackrel{(4)}{\Gamma_{33}^{2}} ; \\
& \text { - } \Gamma_{21}^{2}=\Gamma_{12}^{2}=r^{3}=-\mathrm{g}_{22}^{(2)} \partial_{r} \mathrm{~g}_{22}^{(2)}=\stackrel{(4)}{\Gamma_{21}^{2}} .
\end{aligned}
$$

For $\alpha=3$ :

$$
\Gamma_{\mu \nu}^{3}=\frac{1}{2} g^{3 \lambda}\left(g_{\lambda \mu, \nu}+g_{\nu \lambda, \mu}-g_{\mu \nu, \lambda}\right)=g^{33}\left(g_{3 \mu, \nu}+g_{\nu 3, \mu}\right)
$$

and the components different from zero are

$$
\begin{aligned}
& \text { - } \Gamma_{31}^{3}=\Gamma_{13}^{3}=r^{3} \sin ^{4} \theta=-\mathrm{g}_{33}^{(2)} \partial_{r} \mathrm{~g}_{33}^{(2)}=\Gamma_{31}^{3} \\
& \text { - } \Gamma_{32}^{3}=\Gamma_{23}^{3}=r^{4} \cos \theta \sin ^{3} \theta=-\mathrm{g}_{33}^{(2)} \partial_{\theta} \mathrm{g}_{33}^{(2)}=\stackrel{(4)}{\Gamma_{32}^{3}} .
\end{aligned}
$$


The calculation shows that the Christoffel symbols can be written as

$$
\Gamma_{\mu \nu}^{\alpha}=\stackrel{(2)}{\Gamma_{\mu \nu}^{\alpha}}+\stackrel{(3)}{\Gamma_{\mu \nu}^{\alpha}}+\stackrel{(4)}{\Gamma_{\mu \nu}^{\alpha}}
$$

For future reference, let us list all the non-vanishing components

$$
\begin{aligned}
& \stackrel{(2)}{\Gamma_{01}^{0}}=\frac{1}{2} \partial_{r} \mathrm{~g}_{00}^{(2)} ; \quad \stackrel{(2)}{\Gamma_{11}^{1}}=-\frac{1}{2} \partial_{r} \mathrm{~g}_{11}^{(2)} ; \quad \stackrel{(2)}{\Gamma_{22}^{1}}=\partial_{r} \mathrm{~g}_{22}^{(2)} ; \\
& \stackrel{(2)}{\Gamma_{33}^{1}}=\partial_{r} \mathrm{~g}_{33}^{(2)} ; \quad \Gamma_{00}^{1}=\frac{1}{2} \partial_{r} \mathrm{~g}_{00}^{(2)} \text {; } \\
& \stackrel{(3)}{\Gamma_{00}^{0}}=\frac{1}{2} \partial_{t} \mathrm{~g}_{00}^{(2)} ; \quad \stackrel{(3)}{\Gamma_{11}^{0}}=\frac{1}{2} \partial_{t} \mathrm{~g}_{11}^{(2)} ; \quad \Gamma_{01}^{1}=\frac{1}{2} \partial_{t} \mathrm{~g}_{11}^{(2)} ; \\
& \stackrel{(4)}{\Gamma_{01}^{0}}=\frac{1}{2}\left(-\mathrm{g}_{00}^{(2)} \partial_{r} \mathrm{~g}_{00}^{(2)}+\partial_{r} \mathrm{~g}_{00}^{(4)}\right) ; \quad{\stackrel{(4)}{\Gamma_{11}^{1}}}^{1}=-\frac{1}{2} \mathrm{~g}^{11^{(2)}} \partial_{r} \mathrm{~g}_{11}^{(2)} ; \\
& \stackrel{(4)}{\Gamma_{00}^{1}}=\frac{1}{2}\left(\mathrm{~g}_{11}^{(2)} \partial_{r} \mathrm{~g}_{00}^{(2)}+\partial_{r} \mathrm{~g}_{00}^{(4)}\right) ; \quad \Gamma_{22}^{(4)}=\mathrm{g}_{11}^{(2)} \partial_{r} \mathrm{~g}_{22}^{(2)} ;
\end{aligned}
$$

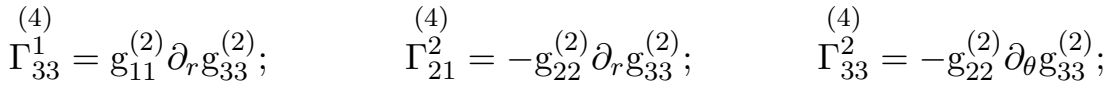

$$
\begin{aligned}
& \stackrel{(4)}{\Gamma_{31}^{3}}=-\mathrm{g}_{33}^{(2)} \partial_{r} \mathrm{~g}_{33}^{(2)} ; \quad \stackrel{(4)}{\Gamma_{32}^{3}}=-\mathrm{g}_{33}^{(2)} \partial_{\theta} \mathrm{g}_{33}^{(2)} \text {. }
\end{aligned}
$$

Using eq. (B.21), the Ricci tensor in eq. (1.7) reads

$$
\begin{aligned}
& R_{\mu \nu}=\partial_{\nu} \stackrel{(2)}{\Gamma}_{\mu \alpha}^{\alpha}+\partial_{\nu} \stackrel{(3)}{\Gamma}_{\mu \alpha}^{\alpha}+\partial_{\nu} \stackrel{(4)}{\Gamma}_{\mu \alpha}^{\alpha}-\partial_{\alpha} \stackrel{(2)}{\Gamma}_{\mu \nu}^{\alpha}-\partial_{\alpha} \stackrel{(3)}{\Gamma}_{\mu \nu}^{\alpha}-\partial_{\alpha} \stackrel{(4)}{\Gamma}_{\mu \nu}^{\alpha}+ \\
& +\left(\begin{array}{c}
(2) \\
\Gamma_{\mu \alpha}^{\lambda}
\end{array} \stackrel{(3)}{\Gamma_{\mu \alpha}^{\lambda}}+\stackrel{(4)}{\Gamma_{\mu \alpha}^{\lambda}}\right)\left(\stackrel{(2)}{\Gamma_{\lambda \nu}^{\alpha}}+\stackrel{(3)}{\Gamma_{\lambda \nu}^{\alpha}}+\stackrel{(4)}{\Gamma_{\lambda \nu}^{\alpha}}\right)+
\end{aligned}
$$

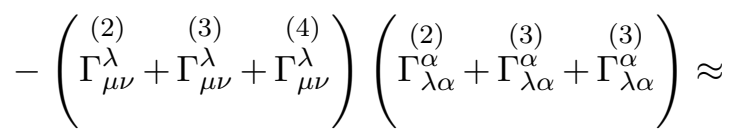

$$
\begin{aligned}
& \approx \partial_{\nu} \stackrel{(2)}{\Gamma}_{\mu \alpha}^{\alpha}-\partial_{\alpha} \stackrel{(2)}{\Gamma}_{\mu \nu}^{\alpha}+\partial_{\nu} \stackrel{(3)}{\Gamma}_{\mu \alpha}^{\alpha}-\partial_{\alpha} \stackrel{(3)}{\Gamma}_{\mu \nu}^{\alpha}+\partial_{\nu} \stackrel{(4)}{\Gamma}_{\mu \alpha}^{\alpha}-\partial_{\alpha} \stackrel{(4)}{\Gamma}_{\mu \nu}^{\alpha}+\stackrel{(2)}{\Gamma_{\mu \alpha}^{\lambda}} \stackrel{(2)}{\Gamma_{\lambda \nu}^{\alpha}}-\stackrel{(2)}{\Gamma_{\mu \nu}^{\lambda}} \stackrel{(2)}{\Gamma_{\lambda \alpha}^{\alpha}},
\end{aligned}
$$

and consequently

$$
\begin{aligned}
& R_{\mu \nu}^{(2)}=\left.\partial_{\nu} \stackrel{(2)}{\Gamma}_{\mu \alpha}^{\alpha}\right|_{\nu \neq 0}-\partial_{k} \Gamma_{\mu \nu}^{k}, \\
& R_{\mu \nu}^{(3)}=\left.\partial_{\nu} \stackrel{(3)}{\Gamma}_{\mu \alpha}^{\alpha}\right|_{\nu \neq 0}-\partial_{k} \stackrel{(3)}{\Gamma}_{\mu \nu}^{k}-\partial_{0} \stackrel{(2)}{\Gamma}_{\mu \nu}^{0}+\left.\partial_{\nu} \stackrel{(2)}{\Gamma}_{\mu \alpha}^{\alpha}\right|_{\nu=0}, \\
& R_{\mu \nu}^{(4)}=\left.\partial_{\nu} \stackrel{(4)}{\Gamma}_{\mu \alpha}^{\alpha}\right|_{\nu \neq 0}-\partial_{\alpha} \stackrel{(4)}{\Gamma}_{\mu \nu}^{\alpha}+\stackrel{(2)}{\Gamma}_{\mu \alpha}^{\lambda} \stackrel{(2)}{\Gamma}_{\lambda \nu}^{\alpha}-\stackrel{(2)}{\Gamma}_{\mu \nu}^{\lambda} \stackrel{(2)}{\Gamma}_{\lambda \alpha}^{\alpha}-\partial_{0} \Gamma_{\mu \nu}^{0}+\left.\partial_{\nu} \stackrel{(3)}{\Gamma}_{\mu \alpha}^{\alpha}\right|_{\nu=0}
\end{aligned}
$$

From eqs. (B.22), (B.23) and (B.24), the components of the Ricci tensor are 
2nd order components: $R_{\mu \nu}^{(2)}=\left.\partial_{\nu} \stackrel{(2)}{\Gamma}_{\mu \alpha}^{\alpha}\right|_{\nu \neq 0}-\partial_{k} \stackrel{(2)}{\Gamma}_{\mu \nu}^{k}$ and

$$
\begin{aligned}
& -R_{00}^{(2)}=-\partial_{k} \stackrel{(2)}{\Gamma}_{00}^{k}=-\partial_{r} \Gamma_{00}^{1}=-\frac{1}{2} \nabla^{2} \mathrm{~g}_{00}^{(2)} \\
& \text { - } R_{i j}^{(2)}=\partial_{j} \stackrel{(2)}{\Gamma}_{i \alpha}^{\alpha}-\partial_{k} \Gamma_{i j}^{k}=\partial_{j} \Gamma_{i 0}^{0}+\partial_{j} \Gamma_{i k}^{k}-\partial_{k} \Gamma_{i j}^{k}= \\
& =\frac{1}{2} \partial_{j} \partial_{i} \mathrm{~g}_{00}^{(2)}-\frac{1}{2} \partial_{j} \partial_{i} \mathrm{~g}_{k k}^{(2)}-\frac{1}{2} \partial_{j} \partial_{k} \mathrm{~g}_{i k}^{(2)}-\frac{1}{2} \partial_{k} \partial_{i} \mathrm{~g}_{k i}^{(2)}+\frac{1}{2} \nabla^{2} \mathrm{~g}_{i j}^{(2)} ;
\end{aligned}
$$

- $R_{i 0}^{(2)}=-\partial_{k} \Gamma_{i 0}^{k}=0$.

3rd order components: $R_{\mu \nu}^{(3)}=\left.\partial_{\nu} \stackrel{(3)}{\Gamma}_{\mu \alpha}^{\alpha}\right|_{\nu \neq 0}-\partial_{k} \stackrel{(3)}{\Gamma}_{\mu \nu}^{k}-\partial_{0} \stackrel{(2)}{\Gamma}_{\mu \nu}^{0}+\left.\partial_{\nu} \stackrel{(2)}{\Gamma}_{\mu \alpha}^{\alpha}\right|_{\nu=0}$, with

- $R_{00}^{(3)}=\partial_{0} \stackrel{(2)}{\Gamma}_{0 \alpha}^{\alpha}-\partial_{k} \Gamma_{00}^{k}-\partial_{0} \stackrel{(2)}{\Gamma}_{00}^{0}=0 ;$

- $R_{i j}^{(3)}=\partial_{j} \stackrel{(3)}{\Gamma}_{i \alpha}^{\alpha}-\partial_{k} \Gamma_{i j}^{k}-\partial_{0} \Gamma_{i j}^{0}=\partial_{j} \Gamma_{i k}^{k}-\stackrel{(3)}{k}_{k}^{k} \Gamma_{i j}^{k}=\partial_{j} \stackrel{(3)}{\Gamma}_{i 1}^{1}-\partial_{1} \Gamma_{i j}^{1}+\partial_{j} \Gamma_{i 2}^{2}+$

$$
-\partial_{2} \stackrel{(3)}{\Gamma}_{i j}^{2}+\partial_{j} \stackrel{(3)}{\Gamma}_{i 3}^{3}-\partial_{3} \stackrel{(3)}{\Gamma}_{i j}^{3}=0
$$

- $R_{i 0}^{(3)}=\partial_{0} \stackrel{(2)}{\Gamma_{i \alpha}^{\alpha}}-\partial_{k} \Gamma_{i 0}^{k}-\partial_{0} \stackrel{(2)}{\Gamma}_{i 0}^{0}=\partial_{0} \stackrel{(2)}{\Gamma}_{i k}^{k}+\partial_{0} \stackrel{(2)}{\Gamma}_{i 0}^{0}-\partial_{k} \Gamma_{i 0}^{k}-\partial_{0} \stackrel{(2)}{\Gamma}_{i 0}^{0}=$

$$
=\partial_{0} \Gamma_{i k}^{k}-\partial_{k} \Gamma_{i 0}^{k}=\frac{1}{2} \partial_{0} \partial_{i} \mathrm{~g}_{j j}^{(2)}-\frac{1}{2} \partial_{k} \partial_{i} \mathrm{~g}_{k 0}^{(3)}-\frac{1}{2} \partial_{k} \partial_{0} \mathrm{~g}_{i k}^{(2)}+\frac{1}{2} \nabla^{2} \mathrm{~g}_{i 0}^{(2)}=0 .
$$

\section{4th order components:}

$$
R_{\mu \nu}^{(4)}=\left.\partial_{\nu} \stackrel{(4)}{\Gamma}_{\mu \alpha}^{\alpha}\right|_{\nu \neq 0}-\partial_{\alpha} \stackrel{(4)}{\Gamma}_{\mu \nu}^{\alpha}+\stackrel{(2)}{\Gamma_{\mu \alpha}^{\lambda}} \stackrel{(2)}{\Gamma_{\lambda \nu}^{\alpha}}-\stackrel{(2)}{\Gamma_{\mu \nu}^{\lambda}} \stackrel{(2)}{\Gamma_{\lambda \alpha}^{\alpha}}-\partial_{0} \stackrel{(3)}{\Gamma}_{\mu \nu}^{0}+\left.\partial_{\nu} \stackrel{(3)}{\Gamma}_{\mu \alpha}^{\alpha}\right|_{\nu=0},
$$

and

$$
\begin{aligned}
& \text { - } R_{00}^{(4)}=-\partial_{k} \stackrel{(4)}{\Gamma}_{00}^{k}+\stackrel{(2)}{\Gamma_{0 \alpha}^{\lambda}} \stackrel{(2)}{\Gamma_{\lambda 0}^{\alpha}}-\stackrel{(2)}{\Gamma_{00}^{\lambda}} \stackrel{(2)}{\Gamma_{\lambda \alpha}^{\alpha}}-\stackrel{(3)}{\partial_{0}} \Gamma_{00}^{0}+\partial_{0} \stackrel{(3)}{\Gamma}_{0 \alpha}^{\alpha}=-\partial_{k} \Gamma_{00}^{k}+ \\
& +\stackrel{(2)}{\Gamma_{00}^{k}} \stackrel{(2)}{\Gamma_{k 0}^{0}}-\stackrel{(2)}{\Gamma_{00}^{k}} \stackrel{(2)}{\Gamma_{k i}^{i}}+\stackrel{(3)}{\partial}_{0}^{\Gamma_{0 k}^{k}}=-\partial_{1} \stackrel{(4)}{\Gamma}_{00}^{1}+\stackrel{(2)}{\Gamma_{00}^{1}} \stackrel{(2)}{\Gamma_{10}^{0}}-\stackrel{(2)}{\Gamma^{1}} \stackrel{(2)}{\Gamma^{1}}{ }_{11}^{1}+\partial_{0} \stackrel{(3)}{\Gamma}_{01}^{1}= \\
& =\frac{1}{2} \partial_{t}^{2} \mathrm{~g}_{k k}^{(2)}-\frac{1}{2} \nabla^{2} \mathrm{~g}_{00}^{(4)}-\frac{1}{2} \mathrm{~g}_{k l}^{(2)} \partial_{k} \partial_{l} \mathrm{~g}_{00}^{(2)}-\frac{1}{2} \partial_{l} \mathrm{~g}_{k l}^{(2)} \partial_{k} \mathrm{~g}_{00}^{(2)}+\frac{1}{4} \partial_{k} \mathrm{~g}_{00}^{(2)} \partial_{k} \mathrm{~g}_{00}^{(2)}+ \\
& +\frac{1}{4} \partial_{k} \mathrm{~g}_{l l}^{(2)} \partial_{k} \mathrm{~g}_{00}^{(2)}=\frac{1}{2} \partial_{t}^{2} \mathrm{~g}_{11}^{(2)}-\frac{1}{2} \nabla^{2} \mathrm{~g}_{00}^{(4)}-\frac{1}{2} \mathrm{~g}_{11}^{(2)} \nabla^{2} \mathrm{~g}_{00}^{(2)}-\frac{1}{2} \partial_{r} \mathrm{~g}_{11}^{(2)} \partial_{r} \mathrm{~g}_{00}^{(2)}+ \\
& +\frac{1}{4} \partial_{r} \mathrm{~g}_{00}^{(2)} \partial_{r} \mathrm{~g}_{00}^{(2)}+\frac{1}{4} \partial_{r} \mathrm{~g}_{11}^{(2)} \partial_{r} \mathrm{~g}_{00}^{(2)}=\frac{1}{2} \partial_{t}^{2} \mathrm{~g}_{11}^{(2)}-\frac{1}{2} \nabla^{2} \mathrm{~g}_{00}^{(4)}-\frac{1}{2} \mathrm{~g}_{11}^{(2)} \nabla^{2} \mathrm{~g}_{00}^{(2)}+ \\
& +\frac{1}{4} \partial_{r} \mathrm{~g}_{00}^{(2)} \partial_{r} \mathrm{~g}_{00}^{(2)}+\frac{1}{2} \partial_{r} \mathrm{~g}_{11}^{(2)} \partial_{r} \mathrm{~g}_{00}^{(2)}
\end{aligned}
$$


- $R_{i 0}^{(4)}=-\partial_{k} \Gamma_{i 0}^{k}+\stackrel{(2)}{\Gamma_{i \alpha}^{\lambda}} \stackrel{(2)}{\Gamma_{\lambda 0}^{\alpha}}-\stackrel{(2)}{\Gamma_{i 0}^{\lambda}} \stackrel{(2)}{\Gamma_{\lambda \alpha}^{\alpha}}-\partial_{0} \Gamma_{i 0}^{0}+\partial_{0} \stackrel{(3)}{\Gamma}_{i \alpha}^{\alpha}=$

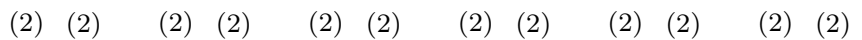

$$
=\Gamma_{i 0}^{\lambda} \Gamma_{\lambda 0}^{0}+\Gamma_{i 1}^{\lambda} \Gamma_{\lambda 0}^{1}+\Gamma_{i 2}^{\lambda} \Gamma_{\lambda 0}^{2}+\Gamma_{i 3}^{\lambda} \Gamma_{\lambda 0}^{3}-\Gamma_{i 0}^{\lambda} \Gamma_{\lambda 0}^{0}-\Gamma_{i 0}^{\lambda} \Gamma_{\lambda 1}^{1}+
$$

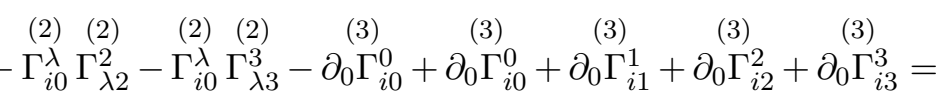

$=\stackrel{(2)}{\Gamma_{i 1}^{\lambda}} \stackrel{(2)}{\Gamma_{\lambda 0}^{1}}-\stackrel{(2)}{\Gamma_{i 0}^{\lambda}} \stackrel{(2)}{\Gamma_{\lambda 1}^{1}}=\stackrel{(2)}{=} \underset{\Gamma_{i 1}^{0}}{\left(\Gamma_{00}^{1}\right.}-\stackrel{(2)}{\Gamma^{0}} \stackrel{(2)}{\Gamma^{1}}{ }_{01}^{1}+\stackrel{(2)}{\Gamma^{1}} \stackrel{(2)}{\Gamma^{1}}{ }_{10}^{1}-\stackrel{(2)}{\Gamma^{1}} \stackrel{(2)}{\Gamma_{11}^{1}}=0 ;$

- $R_{i j}^{(4)}=\partial_{j} \stackrel{(4)}{\Gamma}_{i \alpha}^{\alpha}-\partial_{k} \stackrel{(4)}{\Gamma}_{i j}^{k}+\stackrel{(2)}{\Gamma_{i \alpha}^{\lambda}} \stackrel{(2)}{\Gamma^{\alpha}}{ }_{\lambda j}-\stackrel{(2)}{\Gamma}_{i j}^{\lambda} \stackrel{(2)}{\Gamma_{\lambda \alpha}^{\alpha}}-\partial_{0} \stackrel{(3)}{\Gamma}_{i j}^{0}=$

$$
\begin{aligned}
& =\partial_{j} \Gamma_{i 0}^{0}+\partial_{j} \Gamma_{i 1}^{1}+\partial_{j} \Gamma_{i 2}^{2}+\partial_{j} \stackrel{(4)}{\Gamma}_{i 3}^{3}-\partial_{1} \Gamma_{i j}^{1}-\partial_{2} \Gamma_{i j}^{2}-\partial_{3} \stackrel{(4)}{\Gamma}_{i j}^{3}-\partial_{0} \stackrel{(3)}{\Gamma}_{i j}^{0}+ \\
& \begin{array}{lllllllll}
(2) & (2) \quad(2) \quad(2) \quad(2) & (2) & \text { (2) } & \text { (2) } & \text { (4) } & \text { (4) } & \text { (4) } & \text { (4) }
\end{array} \\
& +\Gamma_{i 0}^{\lambda} \Gamma_{\lambda j}^{0}+\Gamma_{i 1}^{\lambda} \Gamma_{\lambda j}^{1}-\Gamma_{i j}^{\lambda} \Gamma_{\lambda 0}^{0}-\Gamma_{i j}^{\lambda} \Gamma_{\lambda 1}^{1}=\partial_{j} \Gamma_{i 0}^{0}+\partial_{j} \Gamma_{i 1}^{1}+\partial_{j} \Gamma_{i 2}^{2}+\partial_{j} \Gamma_{i 3}^{3}
\end{aligned}
$$

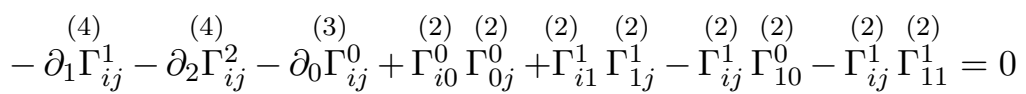

In summary, the non-vanishing components are

$$
\begin{aligned}
& R_{00}^{(2)}=-\frac{1}{2} \nabla^{2} \mathrm{~g}_{00}^{(2)} \\
& R_{i j}^{(2)}=\frac{1}{2} \partial_{j} \partial_{i} \mathrm{~g}_{00}^{(2)}-\frac{1}{2} \partial_{j} \partial_{i} \mathrm{~g}_{k k}^{(2)}-\frac{1}{2} \partial_{j} \partial_{k} \mathrm{~g}_{i k}^{(2)}-\frac{1}{2} \partial_{k} \partial_{i} \mathrm{~g}_{k i}^{(2)}+\frac{1}{2} \nabla^{2} \mathrm{~g}_{i j}^{(2)} \\
& R_{00}^{(4)}=\frac{1}{2} \partial_{t}^{2} \mathrm{~g}_{11}^{(2)}-\frac{1}{2} \nabla^{2} \mathrm{~g}_{00}^{(4)}-\frac{1}{2} \mathrm{~g}_{11}^{(2)} \nabla^{2} \mathrm{~g}_{00}^{(2)}+\frac{1}{4} \partial_{r} \mathrm{~g}_{00}^{(2)} \partial_{r} \mathrm{~g}_{00}^{(2)}+\frac{1}{2} \partial_{r} \mathrm{~g}_{11}^{(2)} \partial_{r} \mathrm{~g}_{00}^{(2)}
\end{aligned}
$$

Eqs. (B.25), (B.26) and (B.27) can be further simplified by assuming harmonic coordinates, that verify

$$
g^{\mu \nu} \Gamma_{\mu \nu}^{\alpha}=0 \Rightarrow\left\{\begin{array}{l}
g^{\mu \nu} \Gamma_{\mu \nu}^{0}=0 \\
g^{\mu \nu} \Gamma_{\mu \nu}^{k}=0
\end{array}\right.
$$

(for more details, see Weinberg (1972)). Eqs. (B.25), (B.26) and (B.27) allow us to compute the Ricci scalar that reads

$$
\begin{aligned}
R & =g^{\mu \nu} R_{\mu \nu}=g^{00} R_{00}+g^{11} R_{11}+g^{22} R_{22}+g^{33} R_{33}= \\
& =-\left(1+g_{00}^{(2)}+g_{00}^{(4)}\right)\left(R_{00}^{(2)}+R_{00}^{(4)}\right)-\left(-1+g_{11}^{(2)}\right) R_{11}^{(2)}-g_{22}^{(2)} R_{22}^{(2)}-g_{33}^{(2)} R_{33}^{(2)}= \\
& =-R_{00}^{(2)}-g_{00}^{(2)} R_{00}^{(2)}-R_{00}^{(4)}+R_{11}^{(2)}-g_{11}^{(2)} R_{11}^{(2)}-g_{22}^{(2)} R_{22}^{(2)}-g_{33}^{(2)} R_{33}^{(2)} .
\end{aligned}
$$

The Ricci scalar can be re-written as

$$
R=R^{(2)}+R^{(4)}
$$


where the 2nd and 4th order components are

$$
R^{(2)}=-R_{00}^{(2)}+R_{11}^{(2)}
$$

and

$$
R^{(4)}=-g_{00}^{(2)} R_{00}^{(2)}-R_{00}^{(4)}-g_{11}^{(2)} R_{11}^{(2)}-g_{22}^{(2)} R_{22}^{(2)}-g_{33}^{(2)} R_{33}^{(2)}
$$

respectively.

Using the vacuum solution of the field equations in Extended Theories of Gravity given by eqs. (1.56) and (1.57), we will restrict our analysis to $f(R)$-Lagrangians that can be Taylor expanded about a certain value $R=R_{0}$, as in eq. (1.62). Carrying the Taylor expansion of the Einstein tensor and its trace, in order to find specific solutions in the weak field limit, we need to expand eqs. (1.56), (1.57) and (1.58) to $\mathcal{O}(0), \mathcal{O}(2)$ e $\mathcal{O}(4)$. At zero order in perturbations we have the condition

$$
\frac{f_{0}}{2} g_{\mu \nu}^{(0)}=0
$$

that automatically implies $f_{0}=0$. Then, the terms in eqs. (1.57) and (1.58) can be rewritten as

$$
\begin{aligned}
\Delta G_{\mu \nu} & =-f_{0}^{\prime \prime}\left\{\partial_{\mu} \partial_{\nu} R-\Gamma_{\mu \nu}^{0} \partial_{t} R-\Gamma_{\mu \nu}^{r} \partial_{r} R-g_{\mu \nu}\left[\left(\partial_{t} g^{00}+g^{00} \partial_{t} \ln \sqrt{-g}\right) \partial_{t} R+\right.\right. \\
& \left.\left.+\left(\partial_{r} g^{11}+g^{11} \partial_{r} \ln \sqrt{-g}\right) \partial_{r} R+g^{00} \partial_{t}^{2} R+g^{11} \partial_{r}^{2} R\right]\right\}
\end{aligned}
$$

and

$$
\begin{aligned}
\Delta G & =3 f_{0}^{\prime \prime}\left[\left(\partial_{t} g^{00}+g^{00} \partial_{t} \ln \sqrt{-g}\right) \partial_{t} R+\left(\partial_{r} g^{11}+g^{11} \partial_{r} \ln \sqrt{-g}\right) \partial_{r} R+\right. \\
& \left.+g^{00} \partial_{t}^{2} R+g^{11} \partial_{r}^{2} R\right]
\end{aligned}
$$

Therefore, at second order of perturbations we obtain

$$
\begin{aligned}
& f_{0}^{\prime} r R^{(2)}-2 f_{0}^{\prime} \partial_{r} g_{t t}^{(2)}+8 f_{0}^{\prime \prime} \partial_{r} R^{(2)}-f_{0}^{\prime} r \partial_{r}^{2} g_{t t}^{(2)}+4 f_{0}^{\prime \prime} r R^{(2)}=0 \\
& f_{0}^{\prime} r R^{(2)}-2 f_{0}^{\prime} \partial_{r} g_{r r}^{(2)}+8 f_{0}^{\prime \prime} \partial_{r} R^{(2)}-f_{0}^{\prime} r \partial_{r}^{2} g_{t t}^{(2)}=0 \\
& 2 f_{0}^{\prime} g_{11}^{(2)}-r\left[f_{0}^{\prime} r R^{(2)}-f_{0}^{\prime} \partial_{r} g_{t t}^{(2)}-f_{0}^{\prime} \partial_{r} g_{r r}^{(2)}+4 f_{0}^{\prime \prime} \partial_{r} R^{(2)}+4 f_{0}^{\prime \prime} r \partial_{r}^{2} R^{(2)}\right]=0 \\
& f_{0}^{\prime} r R^{(2)}+6 f_{0}^{\prime \prime}\left[2 \partial_{r} R^{(2)}+r \partial_{r}^{2} R^{(2)}\right]=0 \\
& 2 g_{11}^{(2)}+r\left[2 \partial_{r} g_{t t}^{(2)}-r R^{(2)}+2 \partial_{r} g_{r r}^{(2)}+r \partial_{r}^{2} g_{t t}^{(2)}\right]=0 .
\end{aligned}
$$

This equations are very general but can be applied to specific theories selecting the corresponding coefficients $f_{i}$ in the Taylor expansion of eq. (1.62). The latter equation, eq. (B.39), corresponding to the trace, closes the system of equations. The solution of 
eqs. (B.34) to (B.39) is

$$
\begin{aligned}
& g_{t t}^{(2)}=\delta_{0}-\frac{\Upsilon}{f_{0}^{\prime} r}-\frac{\delta_{1}(t) e^{-r \sqrt{\xi}}}{3 \xi r}+\frac{\delta_{2}(t) e^{r \sqrt{\xi}}}{6(-\xi)^{3 / 2} r} \\
& g_{r r}^{(2)}=-\frac{\Upsilon}{f_{0}^{\prime} r}+\frac{\delta_{1}(t)[r \sqrt{\xi}+1] e^{-r \sqrt{\xi}}}{3 \xi r}-\frac{\delta_{2}(t)[\xi r+\sqrt{\xi}] e^{r \sqrt{\xi}}}{6 \xi^{2} r} \\
& R^{(2)}=\frac{\delta_{1}(t) e^{-r \sqrt{\xi}}}{r}-\frac{\delta_{2}(t) \sqrt{\xi} e^{r \sqrt{\xi}}}{2 \xi}
\end{aligned}
$$

where $\Upsilon$ is an arbitrary integration constant. The coefficient $\xi=-\frac{f_{0}^{\prime}}{6 f_{0}^{\prime \prime}}$ has units of (length $)^{-2}$; obviously, $f_{0}^{\prime}$ and $f_{0}^{\prime \prime}$ are the expansion coefficients of the Taylor of $f(R)$. The other integration constants, $\delta_{0}$ is dimensionless. The time dependent functions $\delta_{1}(t)$ and $\delta_{2}(t)$ have dimensions (length $)^{-1}$ and (length $)^{-2}$, respectively. This functions can be fixed in the weak field limit. Since in this limit $g_{t t}=1+2 \phi_{\text {grav }}=1+g_{t t}^{(2)}$ then $\delta_{0}=0$ and

$$
\Phi_{\text {grav }}=-\frac{\Upsilon}{2{f^{\prime}{ }_{0} r}^{\prime}}-\frac{\delta_{1}(t) e^{-r \sqrt{-\xi}}}{6 \xi r}+\frac{\delta_{2}(t) e^{r \sqrt{-\xi}}}{12(-\xi)^{3 / 2} r}
$$

In the limit $r \rightarrow \infty$, the potential must go to zero, then the growing term $\frac{\delta_{2}(t) e^{r \sqrt{-\xi}}}{12(-\xi)^{3 / 2} r}$ has to be set to zero by choosing $\delta_{2}(t) \equiv 0$. Finally,

$$
\lim _{r \rightarrow \infty}\left(-\frac{\Upsilon}{2 f^{\prime}{ }_{0} r}-\frac{\delta_{1}(t) e^{-r \sqrt{-\xi}}}{3 \xi r}\right)=-\frac{\Upsilon}{2 f^{\prime}{ }_{0} r},
$$

since in Einstein gravity $f_{0}^{\prime}=1$ then the Newtonian limit implies that $\Upsilon=2 G M$. In our analytic ETGs, $f_{0}^{\prime}$ represent a deviation with respect the standard Newton solution. Eq. (B.43) can be recast as

$$
\Phi_{\text {grav }}=-\left(\frac{G M}{f^{\prime}{ }_{0} r}+\frac{\delta_{1}(t) e^{-r \sqrt{-\xi}}}{6 \xi r}\right),
$$

and introducing the notation of $\sqrt{\xi}=-L^{-1}$, where now $L$ has units of length we arrive

$$
\Phi_{\text {grav }}=-\frac{G M}{f^{\prime}{ }_{0} r}\left(1+\frac{L^{2} f^{\prime}}{6 G M} \delta_{1}(t) e^{-\frac{r}{L}}\right)
$$

where $\delta_{1}(t)$ is an arbitrary function of time that depends by the Taylor coefficients. It is also possible to define an effective gravitational constant $G_{e f f}=G / f_{0}^{\prime}$ and a scale length $L \doteq\left(-\frac{6 f_{0}^{\prime \prime}}{f_{0}^{\prime}}\right)^{1 / 2}$, that depend on the specific $f(R)$ chosen. Eq. (B.46) means that only assuming $f(R)=R$ ones can recover the Newtonian potential, the parameters $f_{0}^{\prime}, f_{0}^{\prime \prime}$, and $\delta_{1}$ can be interpreted as indication of how much an $f(R)$-model is close to GR. Those assumptions need to be tested at Solar System scale where the actual observational constraints have to be matched (Berry and Gair, 2011). Furthermore, 
the parameter $L$ can be related to the effective mass $m=\left(3 / L^{2}\right)^{1 / 2}=\left(-{\frac{f_{0}^{\prime}}{3 f^{\prime \prime}}}^{1 / 2}\right)^{1 / 2}$ and can be interpreted also as an effective length. The eq. (B.46) can be recast as eq. (1.82), and from the comparison of eqs. (B.46) and (1.82) one can obtain $1+\delta=f_{0}^{\prime}$, and $\delta$ is related to $\delta_{1}(t)$ through

$$
\delta_{1}=-\frac{6 G M(r)}{L^{2}} \frac{\delta}{1+\delta},
$$

where $6 G M / L^{2}$ and $\delta_{1}$ are assumed quasi-constant. From the eq. (B.47) comes out the relation $L \propto \sqrt{-\delta /(1+\delta)}$. 


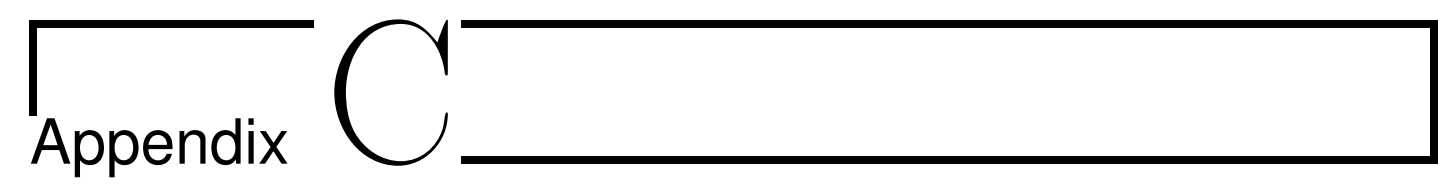

\section{Solutions of unstable modes of a stellar}

\section{system}

In order to compute the integral in eq. (2.14),

$$
W(Z) \equiv \frac{1}{\sqrt{2 \pi}} \int \frac{x e^{-\frac{x^{2}}{2}}}{x-Z} d x
$$

one has to choose a path (Binney and Tremaine, 1994). The contours are shown in Figure C.1, and three cases have to be considered:

1. $\operatorname{Im}(\omega)<0$ (damped solutions): waves with $k>k_{J}$ are damped. The Landau damping is not due to random collisions but to transferring energy to resonant particles. Landau damping does not contribute to the collapse of a selfgravitating structure.

2. $\operatorname{Im}(\omega)=0, \operatorname{Re}(\omega) \neq 0$ : let us assume that the average velocity of a particle is smaller than the phase velocity of the perturbation, the deviations from the above result are small if the imaginary part of $\omega$ is small compared to the real part

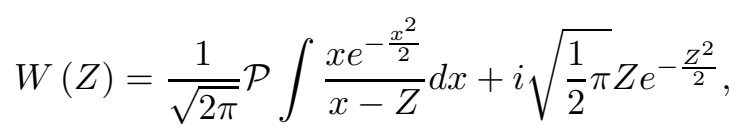

where $Z=\frac{\omega}{k \sigma}$. $\mathcal{P}$ denote the Cauchy principal value of the integral that is real, therefore the imaginary part has to be zero. This condition implies $\omega=0$, that means no undamped solutions in the system. This is the main difference with fluid that support undamped solutions as shown in Fig. 2.1.

3. $\operatorname{Im}(\omega)>0$ (unstable solutions): we compute the real and imaginary part of $\omega$

$$
\operatorname{Re}[W(Z)]=\frac{W(Z)+W^{*}(Z)}{2}=\frac{\operatorname{Re}(Z)}{\sqrt{2 \pi}} \int \frac{x e^{-\frac{x^{2}}{2}} d x}{(x-\operatorname{Re}(Z))^{2}+(\operatorname{Im}(Z))^{2}},
$$



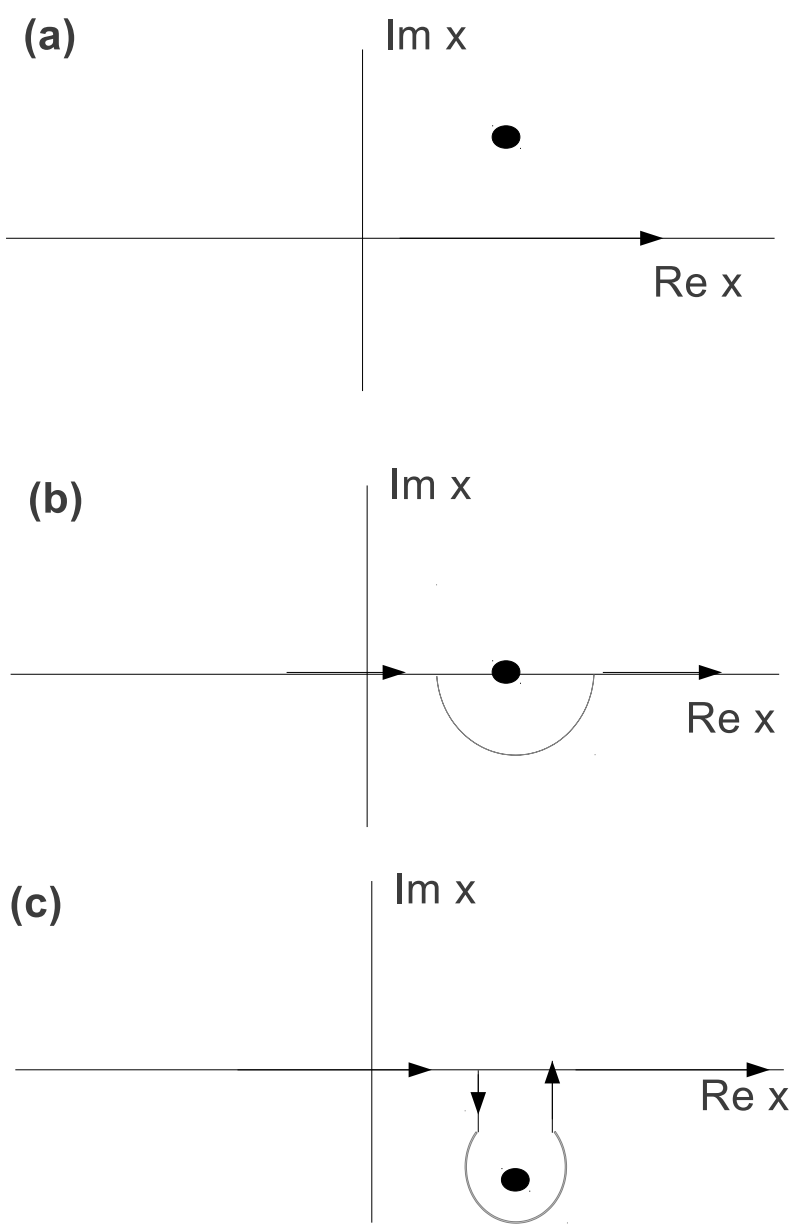

Figure C.1: Landau contours in the complex plane of the $\mathrm{x}$ coordinate.

$$
\operatorname{Im}[W(Z)]=\frac{W(Z)-W^{*}(Z)}{2}=\frac{\operatorname{Im}(Z)}{\sqrt{2 \pi}} \int \frac{x e^{-\frac{x^{2}}{2}} d x}{(x-\operatorname{Re}(Z))^{2}+(\operatorname{Im}(Z))^{2}}
$$

One can write:

$$
1-\frac{k_{J}^{2}}{k^{2}}\{\operatorname{Re}[W(Z)]+i \operatorname{Im}[W(Z)]\}=0,
$$

and setting real part of $\omega$ to be equal to zero $\omega=\omega_{R}+i \omega_{I}=i \omega_{I}$, and being

$$
\mathrm{Z}=\frac{\omega}{k \sigma} \Rightarrow \operatorname{Re}(Z)=0,
$$

One can perform the calculation as following

$$
1-\frac{k_{J}^{2}}{k^{2}}\{\operatorname{Im}[W(Z)]\}=0,
$$


where

$$
\begin{aligned}
& \operatorname{Im}[W(Z)]=\frac{1}{\sqrt{2 \pi}}\left[\int_{0}^{+\infty} \frac{x e^{-\frac{x^{2}}{2}}}{x-i Z_{I}} d x-\int_{0}^{-\infty} \frac{x e^{-\frac{x^{2}}{2}}}{x-i Z_{I}} d x\right]= \\
& =\frac{\sqrt{2}}{\sqrt{\pi}} \int_{0}^{+\infty} \frac{x^{2} e^{-\frac{x^{2}}{2}}}{x^{2}+Z_{I}^{2}} d x,
\end{aligned}
$$

and using the identities $(2.15),(2.16)$, it is obtained

$$
\operatorname{Im}[W(Z)]=\frac{1}{\sqrt{\pi}}\left(\sqrt{\pi}-\pi\left(\frac{\omega_{I}}{\sqrt{2} k \sigma}\right) e^{\left(\frac{\omega_{I}}{\sqrt{2} k \sigma}\right)^{2}}\left[1-\operatorname{erf}\left(\frac{\omega_{I}}{\sqrt{2} k \sigma}\right)\right]\right)
$$

from which one obtain the expression in eq. (2.17)

$$
k^{2}=k_{J}^{2}\left\{1-\sqrt{\pi} \frac{\omega_{I}}{\sqrt{2} k \sigma} e^{\left(\frac{\omega_{I}}{\sqrt{2} k \sigma}\right)^{2}}\left[1-\operatorname{erf}\left(\frac{\omega_{I}}{\sqrt{2} k \sigma}\right)\right]\right\} \text {. }
$$




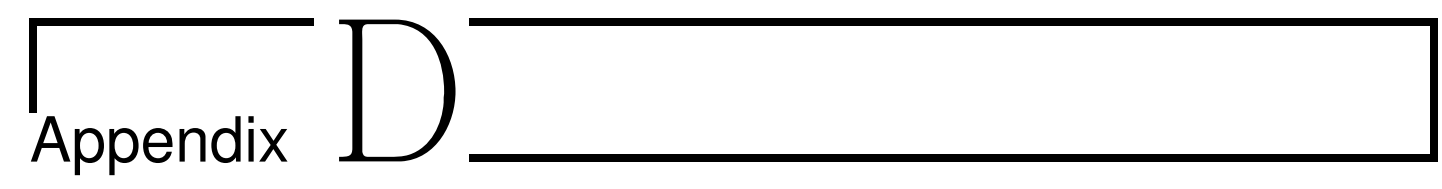

\section{Post-Minkowskian limit and}

\section{quadrupolar emission of gravitational waves in $f(R)$ gravity}

The post-Minkowskian limit is the weak field limit of the theory (Capozziello and De Laurentis, 2011). It does not require velocities to be small as in the Newtonian and Post-Newtonian limits. In the post-Minkowskian limit, one considers small perturbations $h_{\mu \nu}$ on a Minkowskian background $\eta_{\mu \nu}$. The metric element reads

$$
d s^{2}=\left(\eta_{\mu \nu}+h_{\mu \nu}\right) d x^{\mu} d x^{\nu}
$$

with $h_{\mu \nu} \ll \eta_{\mu \nu}\left(\mathcal{O}(h)^{2} \ll 1\right)$. At first order in the perturbation, the field equations (1.75) become

$$
f_{0}^{\prime}\left[R_{\mu \nu}^{(1)}-\frac{R^{(1)}}{2} \eta_{\mu \nu}\right]-f_{0}^{\prime \prime}\left[R_{, \mu \nu}^{(1)}-\eta_{\mu \nu} \square R^{(1)}\right]=\frac{\mathcal{X}}{2} T_{\mu \nu}^{(0)},
$$

and at the zero-order of eqs.(1.75), one gets the condition $f_{0}=0$ as in eq. (B.32).

Notice that the first order in the perturbation corresponds to the zero order in the energy momentum tensor, since Minkowski is a vacuum solution. The corresponding Ricci tensor and Ricci scalar at the first order in the perturbation are

$$
\begin{aligned}
& R_{\mu \nu}^{(1)}=h_{(\mu, \nu) \sigma}^{\sigma}-\frac{1}{2} \square h_{\mu \nu}-\frac{1}{2} h_{, \mu \nu}, \\
& R^{(1)}=h_{\sigma \tau}, \sigma \tau-\square h,
\end{aligned}
$$

with $h=h_{\sigma}^{\sigma}$ the trace of the perturbation tensor (not to be confused with the Hubble coefficient). Using eqs. (D.3) and (D.4) and defining the constant coefficient $\xi=-\frac{f_{0}^{\prime \prime}}{f_{0}^{\prime}}$, 
eq. (D.2) reads

$$
\begin{aligned}
& h_{(\mu, \nu) \sigma}^{\sigma}-\frac{1}{2} \square h_{\mu \nu}-\frac{1}{2} h_{, \mu \nu}-\frac{1}{2}\left(h_{\sigma \tau}{ }^{, \sigma \tau}-\square h\right) \eta_{\mu \nu}+ \\
& +\xi\left(\partial_{\mu \nu}^{2}-\eta_{\mu \nu} \square\right)\left(h_{\sigma \tau}{ }^{, \sigma \tau}-\square h\right)=\frac{\mathcal{X}}{2 f_{0}^{\prime}} T_{\mu \nu}^{(0)} .
\end{aligned}
$$

If we define

$$
\tilde{h}_{\mu \nu}=h_{\mu \nu}-\frac{h}{2} \eta_{\mu \nu}
$$

and imposing the gauge condition

$$
\tilde{h}_{, \mu}^{\mu \nu}=0,
$$

one can rewrite the field equations (and the trace) as

$$
\begin{aligned}
& \square \tilde{h}_{\mu \nu}+\xi\left(\eta_{\mu \nu} \square-\partial_{\mu \nu}^{2}\right) \square \tilde{h}=-\frac{\mathcal{X}}{f_{0}^{\prime}} T_{\mu \nu}^{(0)}, \\
& \square \tilde{h}+3 \xi \square^{2} \tilde{h}=-\frac{\mathcal{X}}{f_{0}^{\prime}} T^{(0)} .
\end{aligned}
$$

To evaluate the energy-momentum tensor, let us assume that the source term $T_{\mu \nu}$ occupies a finite region in space and outside this region $T_{\mu \nu}=0$. Then, from eqs. (D.3), (D.4) and the gauge condition (D.6) one obtains for the vacuum that

$$
R_{\mu \nu}^{(1)}=\square h_{\mu \nu}=0 .
$$

The energy-momentum tensor is defined by eq. (3.37) that, in $f(R)$-gravity, it reads

$$
\begin{aligned}
t_{\alpha}^{\lambda}= & f^{\prime}\left\{\left[\frac{\partial R}{\partial g_{\rho \sigma, \lambda}}-\frac{1}{\sqrt{-g}} \partial_{\xi}\left(\sqrt{-g} \frac{\partial R}{\partial g_{\rho \sigma, \lambda \xi}}\right)\right] g_{\rho \sigma, \alpha}+\frac{\partial R}{\partial g_{\rho \sigma, \lambda \xi}} g_{\rho \sigma, \xi \alpha}\right\}+ \\
& -f^{\prime \prime} R_{, \xi} \frac{\partial R}{\partial g_{\rho \sigma, \lambda \xi}} g_{\rho \sigma, \alpha}-\delta_{\alpha}^{\lambda} f .
\end{aligned}
$$

It is important to stress that the definitions of the energy-momentum tensor in GR and in $f(R)$-gravity are different. In GR, the second order derivatives in the stress-energy tensor give rise to a fourth divergence that can be set to zero, while in ETGs the extra term gives rise to a forth divergence after an integration by parts that produces differential terms of order higher than second.

The energy-momentum tensor (eq. D.11) contains a GR part and some additional terms that come from the $f(R)$ Taylor expansion. To see this, let us write $f \sim f_{0}^{\prime} R+$ $\mathcal{F}(R)$, where $\mathcal{F}$ contains the reminding terms, i.e., $\lim _{R \rightarrow 0} \mathcal{F} \rightarrow R^{2}$. Then

$$
\begin{aligned}
t_{\alpha}^{\lambda}= & f_{0}^{\prime} t_{\left.\alpha\right|_{\mathrm{GR}}}^{\lambda}+\mathcal{F}^{\prime}\left\{\left[\frac{\partial R}{\partial g_{\rho \sigma, \lambda}}-\frac{1}{\sqrt{-g}} \partial_{\xi}\left(\sqrt{-g} \frac{\partial R}{\partial g_{\rho \sigma, \lambda \xi}}\right)\right] g_{\rho \sigma, \alpha}+\frac{\partial R}{\partial g_{\rho \sigma, \lambda \xi}} g_{\rho \sigma, \xi \alpha}\right\}+ \\
& -\mathcal{F}^{\prime \prime} R_{, \xi} \frac{\partial R}{\partial g_{\rho \sigma, \lambda \xi}} g_{\rho \sigma, \alpha}-\delta_{\alpha}^{\lambda} \mathcal{F} .
\end{aligned}
$$


At the first order in the perturbation, eq. (D.12) reads

$$
\begin{aligned}
& t_{\alpha}^{\lambda} \sim t_{\alpha \mid h^{2}}^{\lambda}=f_{0}^{\prime} t_{\left.\alpha\right|_{\mathrm{GR}}}^{\lambda}+f_{0}^{\prime \prime} R^{(1)}\left[\left(-\partial_{\xi} \frac{\partial R^{(1)}}{\partial g_{\rho \sigma, \lambda \xi}}\right) g_{\rho \sigma, \alpha}+\frac{\partial R^{(1)}}{\partial g_{\rho \sigma, \lambda \xi}} g_{\rho \sigma, \xi \alpha}\right]+ \\
& \quad-f_{0}^{\prime \prime} R_{, \xi}^{(1)} \frac{\partial R^{(1)}}{\partial g_{\rho \sigma, \lambda \xi}} g_{\rho \sigma, \alpha}-\frac{1}{2} f_{0}^{\prime \prime} \delta_{\alpha}^{\lambda} R^{(1)^{2}}= \\
& \quad=f_{0}^{\prime} t_{\left.\alpha\right|_{\mathrm{GR}}}^{\lambda}+f_{0}^{\prime \prime}\left[R^{(1)}\left(\frac{\partial R^{(1)}}{\partial g_{\rho \sigma, \lambda \xi}} g_{\rho \sigma, \xi \alpha}-\frac{1}{2} R^{(1)} \delta_{\alpha}^{\lambda}\right)-\partial_{\xi}\left(R^{(1)} \frac{\partial R^{(1)}}{\partial g_{\rho \sigma, \lambda \xi}}\right) g_{\rho \sigma, \alpha}\right],
\end{aligned}
$$

where $R^{(1)}$ is defined as in (D.3) and (D.4). Therefore, as function of $h$ and $\eta$, one gets

$$
\left\{\begin{array}{l}
\frac{\partial R^{(1)}}{\partial g_{\rho \sigma, \lambda \xi}} \sim \frac{\partial R^{(1)}}{\partial h_{\rho \sigma, \lambda \xi}}=\eta^{\rho \lambda} \eta^{\sigma \xi}-\eta^{\lambda \xi} \eta^{\rho \sigma} \\
\frac{\partial R^{(1)}}{\partial g_{\rho \sigma, \lambda \xi}} g_{\rho \sigma, \xi \alpha} \sim h_{, \xi \alpha}^{\lambda \xi}-h_{\alpha}^{, \lambda},
\end{array}\right.
$$

and

$$
\begin{aligned}
t_{\alpha}^{\lambda} \sim f_{0}^{\prime} t_{\left.\alpha\right|_{\mathrm{GR}}}^{\lambda}+f_{0}^{\prime \prime}\left\{\left(h_{, \rho \sigma}^{\rho \sigma}-\square h\right)\left[h_{, \xi \alpha}^{\lambda \xi}-h_{\alpha}^{, \lambda}-\frac{1}{2} \delta_{\alpha}^{\lambda}\left(h_{, \rho \sigma}^{\rho \sigma}-\square h\right)\right]-h_{, \rho \sigma \xi}^{\rho \sigma}{ }_{, \alpha}^{\lambda \xi}+\right. \\
\left.\quad+h_{, \rho \sigma}^{\rho \sigma}{ }^{\lambda} h_{, \alpha}+h_{, \alpha}^{\lambda \xi} \square h_{, \xi}-\square h^{, \lambda} h_{, \alpha}\right\} .
\end{aligned}
$$

As shown in the equation above, $t_{\alpha}^{\lambda}$ consists of a sum of a GR contribution plus an extra term due to the $f(R)$-gravity

$$
t_{\alpha}^{\lambda}=f_{0}^{\prime} t_{\left.\alpha\right|_{\mathrm{GR}}}^{\lambda}+f_{0}^{\prime \prime} t_{\left.\alpha\right|_{f}}^{\lambda}
$$

Let us now assume that the source is very far away (far source approximation); then $h_{\mu \nu}$ can be written as functions of a single scalar variable $t^{\prime}$

$$
t^{\prime}=t-r
$$

where

$$
r^{2}=x_{i} x^{i}
$$

is the distance to the source. Eq. (D.17) can be re-constructed from the coordinate vector $x^{\mu}$ as

$$
t^{\prime}=k_{\lambda} x^{\lambda}
$$

by using the following conditions

$$
\begin{aligned}
& k_{0} \equiv-k^{0} \equiv 1, \quad k_{i} \equiv-\hat{x}_{i}, \\
& \hat{x}_{i} \equiv \frac{x^{i}}{r}
\end{aligned}
$$


Now, let us express the functional dependency by $t^{\prime}=t-r$ as

$$
h_{\mu \nu, \sigma}=\frac{\partial t^{\prime}}{\partial x^{\sigma}} \frac{d h_{\mu \nu}}{d t^{\prime}}=k_{\lambda} \delta_{\sigma}^{\lambda} \dot{h}_{\mu \nu}=k_{\sigma} \dot{h}_{\mu \nu}
$$

where

$$
h_{\mu \nu}=h_{\mu \nu}\left(k_{\lambda} x^{\lambda}\right)=h_{\mu \nu}\left(t^{\prime}\right) .
$$

Here the dot indicate the derivative with respect to the time and $\frac{\partial x^{\lambda}}{\partial x^{\sigma}}=\delta_{\sigma}^{\lambda}$.

Outside the source region,

$$
\square h_{\mu \nu}=0,
$$

and using eq. (D.22), one finds

$$
\square h_{\mu \nu}=h_{\mu \nu, \rho}{ }^{\rho}=\left(k_{\rho} \dot{h}_{\mu \nu}\right),^{\rho}=k_{\rho} k^{\rho} \ddot{h}_{\mu \nu},
$$

that implies

$$
k_{\rho} k^{\rho}=0
$$

Therefore, eq. (D.15) reads

$$
\begin{aligned}
t_{\alpha}^{\lambda}= & f_{0}^{\prime}\left(k^{\lambda} k_{\alpha} \dot{h}^{\rho \sigma} \dot{h}_{\rho \sigma}\right)+f_{0}^{\prime \prime}\left(k_{\rho} k_{\sigma} \ddot{h}^{\rho \sigma} k_{\xi} k_{\alpha} \ddot{h}^{\lambda \xi}-k_{\rho} k_{\sigma} \ddot{h}^{\rho \sigma} k^{\lambda} k_{\alpha} \ddot{h}-\frac{1}{2} k_{\rho} k_{\sigma} \ddot{h}^{\rho \sigma} \delta_{\alpha}^{\lambda} k_{\rho} k_{\sigma} \ddot{h}^{\rho \sigma}+\right. \\
& +\frac{1}{2} k_{\rho} k_{\sigma} \ddot{h}^{\rho \sigma} \delta_{\alpha}^{\lambda} \square h-k_{\xi} k_{\alpha} \ddot{h}^{\lambda \xi} \square h+k^{\lambda} k_{\alpha} \ddot{h} \square h+\frac{1}{2} \delta_{\alpha}^{\lambda} k_{\rho} k_{\sigma} \ddot{h}^{\rho \sigma} \square h-\frac{1}{2} \delta_{\alpha}^{\lambda}(\square h)^{2}+ \\
& \left.-k_{\rho} k_{\sigma} k_{\xi} \dddot{h}^{\rho \sigma} k_{\alpha} \dot{h}^{\lambda \xi}+k_{\rho} k_{\sigma} \dddot{h}^{\rho \sigma} k^{\lambda} k_{\alpha} \dot{h}+k_{\alpha} \dot{h}^{\lambda \xi} \square h_{, \xi}-\square h^{, \lambda} k_{\alpha} \dot{h}\right) .
\end{aligned}
$$

If we denote

$$
\begin{aligned}
& \dot{h}=\eta_{\xi \lambda} \dot{h}^{\lambda \xi}, \quad \ddot{h}=\eta_{\xi \lambda} \ddot{h}^{\lambda \xi}, \\
& k^{\lambda} \eta_{\xi \lambda}=k_{\xi}, \\
& k^{\lambda} k_{\alpha} \ddot{h}=k^{\lambda} k_{\alpha} \eta_{\xi \lambda} \ddot{h}^{\lambda \xi}=k_{\xi} k_{\alpha} \ddot{h}^{\lambda \xi},
\end{aligned}
$$

then we can further simplify eq. (D.27) and

$$
\begin{aligned}
t_{\alpha}^{\lambda}= & f_{0}^{\prime}\left(k^{\lambda} k_{\alpha} \dot{h}^{\rho \sigma} \dot{h}_{\rho \sigma}\right)+f_{0}^{\prime \prime}\left(k_{\rho} k_{\sigma} \ddot{h}^{\rho \sigma} k_{\xi} k_{\alpha} \ddot{h}^{\lambda \xi}-k_{\rho} k_{\sigma} \ddot{h}^{\rho \sigma} k^{\lambda} k_{\alpha} \ddot{h}+\right. \\
& \left.-\frac{1}{2} k_{\rho} k_{\sigma} \ddot{h}^{\rho \sigma} \delta_{\alpha}^{\lambda} k_{\rho} k_{\sigma} \ddot{h}^{\rho \sigma}-k_{\rho} k_{\sigma} k_{\xi} \dddot{h}^{\rho \sigma} k_{\alpha} \dot{h}^{\lambda \xi}+k_{\rho} k_{\sigma} \dddot{h}^{\rho \sigma} k^{\lambda} k_{\alpha} \dot{h}\right) .
\end{aligned}
$$

Notice that the fifth and sixth terms of eq. (D.31) are equal because of the relation,

$$
k_{\rho} k_{\sigma} k^{\lambda} k_{\alpha} \dddot{h}^{\rho \sigma} \dot{h}=k_{\rho} k_{\sigma} k^{\lambda} \dddot{h}^{\rho \sigma} \eta_{\xi \lambda} \dot{h}^{\lambda \xi}=k_{\rho} k_{\sigma} k_{\xi} \dddot{h}^{\rho \sigma} \dot{h}^{\rho \xi} r .
$$


Also, the second and third terms are equal, because of eq. (D.30), so eq. (D.31) reduces to

$$
\begin{aligned}
t_{\alpha}^{\lambda} & =f_{0}^{\prime}\left(k^{\lambda} k_{\alpha} \dot{h}^{\rho \sigma} \dot{h}_{\rho \sigma}\right)-\frac{1}{2} f_{0}^{\prime \prime}\left(k_{\rho} k_{\sigma} \ddot{h}^{\rho \sigma} \delta_{\alpha}^{\lambda} k_{\rho} k_{\sigma} \ddot{h}^{\rho \sigma}\right)= \\
& =f_{0}^{\prime}\left(k^{\lambda} k_{\alpha} \dot{h}^{\rho \sigma} \dot{h}_{\rho \sigma}\right)-\frac{1}{2} f_{0}^{\prime \prime}\left(k_{\rho} k_{\sigma} \ddot{h}^{\rho \sigma} \eta^{\lambda \xi} \eta_{\xi \alpha} k_{\rho} k_{\sigma} \ddot{h}^{\rho \sigma}\right)
\end{aligned}
$$

Finally, the energy-momentum tensor assume the following form

$$
t_{\alpha}^{\lambda}=\underbrace{f_{0}^{\prime} k^{\lambda} k_{\alpha}\left(\dot{h}^{\rho \sigma} \dot{h}_{\rho \sigma}\right)}_{G R}-\underbrace{\frac{1}{2} f_{0}^{\prime \prime} \delta_{\alpha}^{\lambda}\left(k_{\rho} k_{\sigma} \ddot{h}^{\rho \sigma}\right)^{2}}_{f(R)} .
$$

Let us remark that the order of derivatives has increased in two degrees reflecting the fact that in the metric approach $f(R)$-gravity is described by fourth-order field equations.

At this point, one can use eq.(D.34) to compute the energy radiated $(d E / d t)$. Like in GR we assume that $h_{\mu \nu}$ is a plane wave, we can compute the energy radiated averaging over an interval equal to or greater than orbital period $T$ (Landau and Lifshitz (1962), Maggiore (2007)). The average flux of energy through a surface of area $r^{2} d \Omega$ in the direction $\hat{x}$ is

$$
\left\langle\frac{d E}{d t}\right\rangle=r^{2} d \Omega \hat{x}^{i}\left\langle t^{0 i}\right\rangle
$$

therefore the eq. D.34 becomes

$$
\left\langle t_{\alpha}^{\lambda}\right\rangle=\left\langle f_{0}^{\prime} k^{\lambda} k_{\alpha}\left(\dot{h}^{\rho \sigma} \dot{h}_{\rho \sigma}\right)-\frac{1}{2} f_{0}^{\prime \prime} \delta_{\alpha}^{\lambda}\left(k_{\rho} k_{\sigma} \ddot{h}^{\rho \sigma}\right)^{2}\right\rangle
$$

\section{Multipole Analysis of radiated energy.}

As in GR, we can write $h_{\mu \nu}$ in terms of the stress-energy tensor

$$
h_{\mu \nu}(\vec{x}, t) \simeq 4 \int d^{3} \vec{x}^{\prime} \frac{T_{\mu \nu}\left(\vec{x}^{\prime}, t-\left|\vec{x}^{\prime}-\vec{x}\right|\right)}{\left|\vec{x}^{\prime}-\vec{x}\right|},
$$

therefore, expanding the time dependence of $h_{\mu \nu}$ in a Taylor series we obtain

$$
\begin{aligned}
h^{\mu \nu}(\vec{x}, t)= & \frac{4}{r}\left[\int d^{3} \vec{x}^{\prime} T^{\mu \nu}\left(\vec{x}^{\prime}, t^{\prime}\right)+\hat{x} \int d^{3} \vec{x}^{\prime} \vec{x}^{\prime} \frac{\partial T^{\mu \nu}(\vec{x}, t)}{\partial t^{\prime}}+\right. \\
& \left.+\frac{1}{2} \int d^{3} \vec{x}^{\prime}\left(\hat{x} \cdot \vec{x}^{\prime}\right)^{2} \frac{\partial^{2} T^{\mu \nu}(\vec{x}, t)}{\partial t^{\prime 2}}\right],
\end{aligned}
$$

where we have used the far source approximation

$$
\left|\vec{x}^{\prime}-\vec{x}\right|^{-1} \simeq \frac{1}{r}
$$


and

$$
\left|\vec{x}^{\prime}-\vec{x}\right| \simeq r-\hat{x} \cdot \vec{x}^{\prime}
$$

for $r>>\left|\vec{x}^{\prime}\right|$. Using the definition of the momenta of the mass-energy distribution in eqs. (3.16), (3.17), (3.18) and the conservation law $T^{\mu \nu}{ }_{, \nu}=0$, we obtain Weinberg (1972), Maggiore (2007)

$$
\begin{gathered}
\int d^{3} \vec{x} T^{i j}(\vec{x}, t)=\frac{1}{2} \frac{\partial^{2}}{\partial t^{2}} \int d^{3} \vec{x} x^{j} x^{k} T^{00}(\vec{x}, t)=\frac{1}{2} \ddot{M}^{i j}(t), \\
\int d^{3} \vec{x} T^{0 i}(\vec{x}, t)=\frac{\partial}{\partial t} \int d^{3} \vec{x} x^{k} T^{00}(\vec{x}, t)=\dot{M}^{i}(t), \\
\frac{\partial}{\partial t} \int d^{3} \vec{x} x^{k} T^{i 0}(\vec{x}, t)=\int d^{3} \vec{x} T^{i j}(\vec{x}, t)=\frac{1}{2} \ddot{M}^{i j}(t),
\end{gathered}
$$

Let us rewrite the $h^{\mu \nu}$ components, eq. (D.38), in terms of the momenta eqs. ((3.16)(3.18)):

- for the 00-component one has

$$
\begin{aligned}
h^{00}(\vec{x}, t)= & 4 \frac{1}{r}\left[\int d^{3} \vec{x}^{\prime} T^{00}\left(\vec{x}^{\prime}, t^{\prime}\right)+\hat{x}_{i} \frac{\partial}{\partial t^{\prime}} \int d^{3} \vec{x}^{\prime} x^{\prime i} T^{00}\left(\vec{x}^{\prime}, t^{\prime}\right)+\right. \\
& \left.+\frac{1}{2} \hat{x}_{i} \hat{x}_{j} \frac{\partial^{2}}{\partial t^{2}} \int d^{3} \vec{x}^{\prime} x^{i} x^{\prime j} T^{00}\left(\vec{x}^{\prime}, t^{\prime}\right)+\ldots\right]
\end{aligned}
$$

that it can be written as

$$
h^{00}(\vec{x}, t)=\frac{4}{f_{0}^{\prime}} \frac{1}{r}\left[M\left(t^{\prime}\right)+\hat{x}_{i} \dot{M}^{i}\left(t^{\prime}\right)+\frac{1}{2} \hat{x}_{i} \hat{x}_{j} \ddot{M}^{i j}\left(t^{\prime}\right)\right] .
$$

- For the $0 i$-components we only have to consider two terms on the expansion of eq.(D.38) in order to include terms up to the second order in the momentum

$$
h^{0 i}(\vec{x}, t)=4 \frac{1}{r}\left[\int d^{3} \vec{x}^{\prime} T^{0 i}\left(\vec{x}^{\prime}, t^{\prime}\right)+\hat{x}_{k} \frac{\partial}{\partial t^{\prime}} \int d^{3} \vec{x}^{\prime} x^{\prime k} T^{0 i}\left(\vec{x}^{\prime}, t^{\prime}\right)\right],
$$

By using eqs.(D.42) and (D.43), one obtains

$$
h^{0 i}(\vec{x}, t)=4 \frac{1}{r}\left[\dot{M}^{i}\left(t^{\prime}\right)+\frac{1}{2} \hat{x}_{k} \ddot{M}^{i k}\left(t^{\prime}\right)\right] .
$$

- For the $i j$-components we only need to consider the first term on eq.(D.38)

$$
h^{i j}(\vec{x}, t)=2 \frac{1}{r} \ddot{M}^{i j}\left(t^{\prime}\right) .
$$


Next, using the conservation of the energy momentum-tensor, we obtain

$$
\dot{M}=0, \quad \ddot{M}^{k}=0,
$$

and from eqs.(D.45), (D.47) and (D.48) we obtain

$$
\begin{aligned}
\dot{h}^{00} & =2 \frac{1}{r} \hat{x}_{i} \hat{x}_{j} \dddot{M}^{i j}, & \ddot{h}^{00} & =2 \frac{1}{r} \hat{x}_{i} \hat{x}_{j} \dddot{M}^{i j}, \\
\dot{h}^{0 i} & =2 \frac{1}{r} \hat{x}_{k} \dddot{M}^{i k}, & \ddot{h}^{0 i} & =2 \frac{1}{r} \hat{x}_{k} \dddot{M}^{i k}, \\
\dot{h}^{i j} & =2 \frac{1}{r} \dddot{M}^{i j}, & \ddot{h}^{i j} & =2 \frac{1}{r} \dddot{M}^{i j}
\end{aligned}
$$

In order to evaluate eq.(D.36) let us remind the reader that

$$
\dot{h}^{\rho \sigma} \dot{h}_{\rho \sigma}=\dot{h}^{00} \dot{h}_{00}+2 \dot{h}^{0 i} \dot{h}_{0 i}+\dot{h}^{i j} \dot{h}_{i j} .
$$

and

$$
\ddot{h}^{\rho \sigma} \ddot{h}_{\rho \sigma}=\ddot{h}^{00} \ddot{h}_{00}+2 \ddot{h}^{0 i} \ddot{h}_{0 i}+\ddot{h}^{i j} \ddot{h}_{i j}
$$

Introducing eqs. (D.50)-(D.52) onto eqs. (D.53) and (D.54) we obtain

$$
\dot{h}^{\rho \sigma} \dot{h}_{\rho \sigma}=\frac{4}{r^{2}}\left[\left(\hat{x}_{i} \hat{x}_{j} \dddot{M}^{i j}\right)^{2}-2\left(\hat{x}_{k} \dddot{M}^{i k}\right)\left(\hat{x}_{j} \dddot{M}^{i j}\right)+\left(\dddot{M}^{i j} \dddot{M}_{i j}\right)\right] \text {. }
$$

In complete analogy, replacing eqs. (D.50)-(D.52) onto eqs. (D.53) and (D.54) we obtain

$$
\ddot{h}^{\rho \sigma} \ddot{h}_{\rho \sigma}=\frac{4}{r^{2}}\left[\left(\hat{x}_{i} \hat{x}_{j} \dddot{M}^{i j}\right)^{2}-2\left(\hat{x}_{k} \dddot{M}^{i k}\right)\left(\hat{x}_{j} \dddot{M}^{i j}\right)+\left(\dddot{M}^{i j} \dddot{M}_{i j}\right)\right] .
$$

Finally, replacing eqs. (D.55) and (D.56) into eq.(D.36), one can rewrite the energymomentum tensor as

$$
\begin{aligned}
\left\langle t_{\alpha}^{\lambda}\right\rangle= & \left\langle f_{0}^{\prime} k^{\lambda} k_{\alpha} \frac{4}{r^{2}}\left[\left(\hat{x}_{i} \hat{x}_{j} \dddot{M}^{i j}\right)^{2}-2\left(\hat{x}_{k} \dddot{M}^{i k}\right)\left(\hat{x}_{j} \dddot{M}^{i j}\right)+\left(\dddot{M}^{i j} \dddot{M}_{i j}\right)\right]+\right. \\
& \left.-f_{0}^{\prime \prime} \delta_{\alpha}^{\lambda}\left(k_{\rho} k_{\sigma}\right)^{2} \frac{2}{r^{2}}\left[\left(\hat{x}_{i} \hat{x}_{j} \dddot{M}^{i j}\right)^{2}-2\left(\hat{x}_{k} \dddot{M}^{i k}\right)\left(\hat{x}_{j} \dddot{M}^{i j}\right)+\left(\dddot{M}^{i j} \dddot{M}_{i j}\right)\right]\right\rangle
\end{aligned}
$$

Using eq. (D.35) and integrating over all directions

$$
\left\langle\frac{d E}{d t}\right\rangle_{(\text {total })}=r^{2} \int d \Omega \hat{x}^{i}\left\langle t^{0 i}\right\rangle
$$

Let us notice that

$$
\hat{x}^{\alpha}\left\langle t^{0 i}\right\rangle=\hat{x}^{i} k^{0} k^{i}[\ldots]=\hat{x}^{i}(-1)\left(-\hat{x}^{i}\right)[\ldots]=[\ldots]
$$


which simplifies the evaluation of eq.(D.58). In addition,

$$
\int d \Omega \hat{x}^{i} \hat{x}^{j}=\frac{4 \pi}{3} \delta_{i j},
$$

and

$$
\int d \Omega \hat{x}^{i} \hat{x}^{j} \hat{x}^{l} \hat{x}^{m}=\frac{4 \pi}{15}\left(\delta_{i j} \delta_{l m}-\delta_{i l} \delta_{j m}\right),
$$

we finally arrive to

$$
\underbrace{\left\langle\frac{d E}{d t}\right\rangle}_{\text {(total })}=\frac{G}{60}\langle\underbrace{f_{0}^{\prime}\left(\dddot{Q}^{i j} \dddot{Q}_{i j}\right)}_{G R}-\underbrace{f_{0}^{\prime \prime}\left(\dddot{Q}^{i j} \dddot{Q}_{i j}\right)}_{f(R)}\rangle .
$$




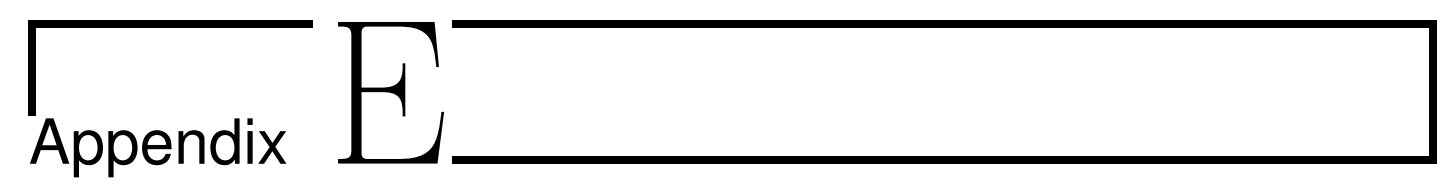

\section{Relativistic corrections of thermal}

\section{Sunyaev-Zeldovich effect}

In clusters with temperatures $k_{B} T_{e}>10 \mathrm{keV}$ relativistic effects become relevant. They can be included in our formalism by properly modifying eq. (4.3) (Itoh et al. (1998), Nozawa et al. (1998)). In the non-relativistic limit, the frequency dependence of the TSZ effect is

$$
g_{0}(x)=x \operatorname{coth}(x / 2)-4
$$

where the reduced frequency is $x=\frac{h \nu(z)}{k_{B} T(z)}$. In the relativistic case, extra terms appear

$$
g(x)=g_{0}(x)+x Y_{1}+x^{2} Y_{2}+x^{3} Y_{3}+x^{4} Y_{4}
$$

Each term of this series have an approximated analytic expression (Itoh et al. (1998), Nozawa et al. (1998)) that simplifies introducing relativistic corrections. The terms above are

$$
\begin{aligned}
& Y_{1}=-10+\frac{47}{2} \tilde{X}-\frac{42}{5} \tilde{X}^{2}+\frac{7}{10} \tilde{X}^{3}+\tilde{S}^{2} \frac{(-21+7 \tilde{X})}{5} \\
& Y_{2}=-\frac{15}{2}+\frac{1023}{8} \tilde{X}-\frac{868}{5} \tilde{X}^{2}+\frac{329}{5} \tilde{X}^{3}-\frac{44}{5} \tilde{X}^{4}+\frac{11}{30} \tilde{X}^{5}+\tilde{S}^{2}\left(-\frac{434}{5}+\right. \\
& \left.+\frac{658}{5} \tilde{X}-\frac{242}{5} \tilde{X}^{2}+\frac{143}{30} \tilde{X}^{3}\right)
\end{aligned}
$$




$$
\begin{aligned}
& Y_{3}=\frac{15}{2}+\frac{2505}{8} \tilde{X}-\frac{7098}{5} \tilde{X}^{2}+\frac{1425}{3} \tilde{X}^{3}-\frac{18594}{35} \tilde{X}^{4}+\tilde{S}^{2}\left(-\frac{7098}{10}+\right. \\
& \left.+\frac{14253}{5} \tilde{X}-\frac{102267}{35} \tilde{X}^{2}+\frac{156767}{140} \tilde{X}^{3}-\frac{1216}{7} \tilde{X}^{4}+\frac{64}{7} \tilde{X}^{5}\right)+\tilde{S}^{4}\left(-\frac{18594}{35}+\right. \\
& \left.+\frac{20500}{28} \tilde{X}-\frac{1920}{7} \tilde{X}^{2}+\frac{1024}{35} \tilde{X}^{3}\right)+\tilde{S}^{6}\left(-\frac{544}{21}+\frac{992}{105} \tilde{X}\right) \\
& Y_{4}=-\frac{135}{32}+\frac{30375}{128} \tilde{X}-\frac{62391}{10} \tilde{X}^{2}+\frac{614727}{40} \tilde{X}^{3}-\frac{124389}{10} \tilde{X}^{4}+\frac{355703}{8} \tilde{X}^{5}+ \\
& -\frac{16568}{21} \tilde{X}^{6}+\frac{7516}{105} \tilde{X}^{7}-\frac{22}{7} \tilde{X}^{8}+\frac{11}{210} \tilde{X}^{9}+\tilde{S}^{2}\left(-\frac{62391}{20}+\frac{614727}{20} \tilde{X}_{-}\right. \\
& \left.-\frac{1368279}{20} \tilde{X}^{2}+\frac{4624139}{80} \tilde{X}^{3}-\frac{157396}{7} \tilde{X}^{4}+\frac{30064}{7} \tilde{X}^{5}-\frac{2717}{7} \tilde{X}^{6}+\frac{2761}{210} \tilde{X}^{7}\right)+ \\
& +\tilde{S}^{4}\left(-\frac{124389}{10}+\frac{6046951}{160} \tilde{X}-\frac{248520}{7} \tilde{X}^{2}+\frac{481024}{35} \tilde{X}^{3}-\frac{15972}{7} \tilde{X}^{4}+\frac{18689}{140} \tilde{X}^{5}\right)+ \\
& +\tilde{S}^{6}\left(-\frac{70414}{21}+\frac{465992}{105} \tilde{X}-\frac{11792}{7} \tilde{X}^{2}+\frac{19778}{105} \tilde{X}^{3}\right)+\tilde{S}^{8}\left(-\frac{682}{7}+\frac{7601}{210} \tilde{X}\right)
\end{aligned}
$$

where

$$
\tilde{X}=x \operatorname{coth}\left(\frac{x}{2}\right)
$$

and

$$
\tilde{S}=\frac{x}{\sinh (x)} .
$$




\section{List of Figures}

1.1 Energy density contents of the Universe . . . . . . . . . . . . . . 9

1.2 Evolution of the energy components in units of the critical density . . . 10

1.3 SN Union 2.1 data set and some theoretical luminosity distances . . . . 13

1.4 Variation of the CMB power spectrum with cosmological parameters . . 16

1.5 Differences in resolution between WMAP and Planck maps . . . . . . 18

1.6 Modified Newtonian potential at Solar System scale. . . . . . . . . . 29

2.1 The dispersion relation of Jeans instability in GR . . . . . . . . . . 35

2.2 The dispersion relation of Jeans instability in ETGs . . . . . . . . . . . 38

$2.3 M_{J}-T$ relation for the interstellar medium . . . . . . . . . . . 41

3.1 Motion of point particles due to the interaction with a GW . . . . . 49

3.2 Time variation of the orbital period as a function of $f_{0}^{\prime \prime} \ldots \ldots 56$

3.3 Analysis of the binary system PSR $J 0348+0432 \ldots \ldots$. . . . . . . 58

4.1 Spectral dependence of SZ effect in the cluster . . . . . . . . . . . . 64

4.2 Ratios $r_{500} / r_{v i r}$ and overdensity mass to virial mass $M_{v i r} / M_{500} \ldots \ldots 8$

4.3 Integration of the cluster pressure profile in ETGs . . . . . . . . . . 70

4.4 Pressure profiles integrated along the line of sight for the Coma cluster . 71

4.5 Distribution on the sky of the X-ray clusters . . . . . . . . . . . 73

4.6 CMB maps SMICA and NILC, galactic and point source mask, and cluster template . . . . . . . . . . . . . . . . . . 74

4.7 Averaged temperature anisotropy profile . . . . . . . . . . . . . 75

4.8 Pressure profiles of clusters in $f(R)$ gravity . . . . . . . . . . . 76

4.9 Confidence contours for pairs of parameters of Model A . . . . . . . 77

4.10 Confidence contours for pairs of parameters of Model B . . . . . . . . 78

5.1 Parameterizations of the CMB temperature dependence with redshift . . 84

5.2 Ratio Method and spectral frequency dependence . . . . . . . . . . . . 85 
5.3 Ratio method and spectral frequency dependence on subtracted CMB maps . . . . . . . . . . . . . . . . . . . . . 86

5.4 WMAP 7 yrs data: Kp0 Mask . . . . . . . . . . . . . . . . . 88

5.5 TSZ template containing the profile of 623 clusters $\ldots$. . . . . . . . 89

5.6 Pressure profile and Comptonization parameter . . . . . . . . . . . . 90

5.7 TSZ and KSZ templates from N-Body hydrodynamical simulations . . . 90

5.8 Comparison between X-Ray and simulated clusters . . . . . . . . . . . 91

5.9 Likelihood function of the ratio method for pipeline $A$ on N-body simulation . . . . . . . . . . . . . . . . . . . . . . . . 94

5.10 Effect of CMB residual for pipeline $A$ on N-body simulation . . . . . . . 95

5.11 Likelihood function of the ratio method for pipeline $B$ on N-body simulation . . . . . . . . . . . . . . . . . . 95

5.12 Effect of the beam dilution on the spectral dependence of the TSZ effect 97

5.13 Deconvolution Factors . . . . . . . . . . . . . . . . . . . . . . 98

5.14 Likelihood function for different cluster subsets in pipeline A . . . . . . . 100

5.15 Likelihood function with the fit method in pipeline B . . . . . . . . . . 100

5.16 Likelihood functions for the fit method using pipeline (B) on real clusters102

C.1 Landau contours in the complex plane of the $\mathrm{x}$ coordinate. . . . . . . . 122 


\section{List of Tables}

1.1 Scaling in an FLRW Universe . . . . . . . . . . . . . 8

1.2 WMAP Mission characteristics. . . . . . . . . . . . . . . 17

1.3 Technical characteristics of Planck channels. . . . . . . . . . . . . 17

1.4 Best-fit cosmological parameters from WMAP 9yr data and Planck 2013

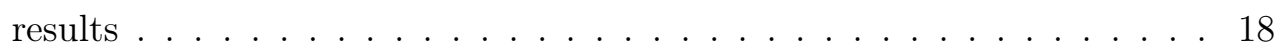

$1.5 f(R)$ models having viable cosmological solutions. . . . . . . . . . . 25

2.1 Jeans masses for different astrophysical systems . . . . . . . . . . . . . . 40

2.2 Physical properties and Jeans masses for a sample of molecular clouds . 42

3.1 Observational data on binary pulsars . . . . . . . . . . . . . 55

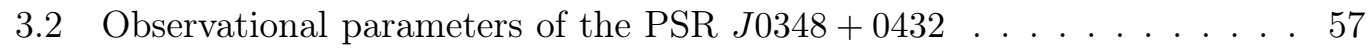

4.1 Magnitudes (observed and derived) of X-ray clusters . . . . . . . . 66

4.2 Parameters of the universal pressure profile . . . . . . . . . . . . . 69

4.3 Results from cluster profile on ETGs. . . . . . . . . . . . . . . . 78

5.1 Results of the ratio method for pipeline $A$ and $B$ on N-body simulation 96

5.2 Deconvolution Factors . . . . . . . . . . . . . . . . . . . . . 99

5.3 Results pipelines (A) and (B) on clusters selected from a N-body simulation . . . . . . . . . . . . . . . . . . . . 101

5.4 Results for $\beta(=2 / 3)$-profile . . . . . . . . . . . . . . . . 101

5.5 Results for universal profile . . . . . . . . . . . . . . . . . . 102

A.1 Physical constants of interest. . . . . . . . . . . . . . . . . . 109

A.2 Astrophysical and Cosmological quantities of interest. . . . . . . . . . 110 


\section{Bibliography}

Abbott, B. P., Abbott, R., Adhikari, R., Ajith, P., Allen, B., Allen, G., Amin, R. S., Anderson, S. B., Anderson, W. G., Arain, M. A., and et al. (2009). Ligo: the laser interferometer gravitational-wave observatory. Reports on Progress in Physics, 72(7):076901.

Allemandi, G., Francaviglia, M., Ruggiero, M., and Tartaglia, A. (2005). General Relativity and Gravitation, 37:1891-1904.

Amanullah, R., Lidman, C., Rubin, D., Aldering, G., Astier, P., Barbary, K., Burns, M. S., Conley, A., Dawson, K. S., Deustua, S. E., Doi, M., Fabbro, S., Faccioli, L., Fakhouri, H. K., Folatelli, G., Fruchter, A. S., Furusawa, H., Garavini, G., Goldhaber, G., Goobar, A., Groom, D. E., Hook, I., Howell, D. A., Kashikawa, N., Kim, A. G., Knop, R. A., Kowalski, M., Linder, E., Meyers, J., Morokuma, T., Nobili, S., Nordin, J., Nugent, P. E., Östman, L., Pain, R., Panagia, N., Perlmutter, S., Raux, J., Ruiz-Lapuente, P., Spadafora, A. L., Strovink, M., Suzuki, N., Wang, L., Wood-Vasey, W. M., Yasuda, N., and Supernova Cosmology Project, T. (2010). ApJ, 716:712.

Amaro-Seoane, P., Aoudia, S., Babak, S., Binétruy, P., Berti, E., Bohé, A., Caprini, C., Colpi, M., Cornish, N. J., Danzmann, K., Dufaux, J.-F., Gair, J., Hinder, I., Jennrich, O., Jetzer, P., Klein, A., Lang, R. N., Lobo, A., Littenberg, T., McWilliams, S. T., Nelemans, G., Petiteau, A., Porter, E. K., Schutz, B. F., Sesana, A., Stebbins, R., Sumner, T., Vallisneri, M., Vitale, S., Volonteri, M., Ward, H., and Wardell, B. (2013). eLISA: Astrophysics and cosmology in the millihertz regime. GW Notes, $6: 4-110$.

Amendola, L. and Euclid Theory Working Group (2013). Cosmology and fundamental physics with the euclid satellite. Living Reviews in Relativity, 16(6).

Amendola, L. and Tsujikawa, S. (2008). Physics Letters B, 660:125-132. 
Anderson, J. D., Laing, P. A., Lau, E. L., Liu, A. S., Nieto, M. M., and Turyshev, S. G. (2002). Phys. Rev. D, 65(8):082004.

Anderson, L. D., Bania, T. M., Jackson, J. M., Clemens, D. P., Heyer, M., Simon, R., Shah, R. Y., and Rathborne, J. M. (2009). ApJS, 181:255-271.

Anderson, S., Gorham, P., Kulkarni, S., and Prince, T. (1990). Nature, 346:42-44.

Antoniadis, J., Freire, P. C. C., Wex, N., Tauris, T. M., Lynch, R. S., van Kerkwijk, M. H., Kramer, M., Bassa, C., Dhillon, V. S., Driebe, T., Hessels, J. W. T., Kaspi, V. M., Kondratiev, V. I., Langer, N., Marsh, T. R., McLaughlin, M. A., Pennucci, T. T., Ransom, S. M., Stairs, I. H., van Leeuwen, J., Verbiest, J. P. W., and Whelan, D. G. (2013). Science, 340:448.

Arnaud, M., Pratt, G. W., Piffaretti, R., Böhringer, H., Croston, J. H., and Pointecouteau, E. (2010). A\&A, 517:A92.

Arzoumanian, Z., Fruchter, A., and Taylor, J. (1994). ApJ, 426:L85-L88.

Astier, P., Guy, J., Regnault, N., Pain, R., Aubourg, E., Balam, D., Basa, S., Carlberg, R. G., Fabbro, S., Fouchez, D., Hook, I. M., Howell, D. A., Lafoux, H., Neill, J. D., Palanque-Delabrouille, N., Perrett, K., Pritchet, C. J., Rich, J., Sullivan, M., Taillet, R., Aldering, G., Antilogus, P., Arsenijevic, V., Balland, C., Baumont, S., Bronder, J., Courtois, H., Ellis, R. S., Filiol, M., Gonçalves, A. C., Goobar, A., Guide, D., Hardin, D., Lusset, V., Lidman, C., McMahon, R., Mouchet, M., Mourao, A., Perlmutter, S., Ripoche, P., Tao, C., and Walton, N. (2006). A\&A, 447:31-48.

Atrio-Barandela, F., Kashlinsky, A., Kocevski, D., and Ebeling, H. (2008). ApJ, 675:L57.

Bahcall, J. and Wolf, R. (1968). ApJ, 152:701.

Bailes, M., Harrison, P. A., Lorimer, D. R., Johnston, S., Lyne, A. G., Manchester, R. N., D'Amico, N., Nicastro, L., Tauris, T. M., and Robinson, C. (1994). ApJL, 425:L41-L44.

Barrow, J. and Ottewill, A. (1983). J. Phys. A, 16:2757.

Battistelli, E., De Petris, M., Lamagna, L., Melchiorri, F., Palladino, E., Savini, G., Cooray, A., Melchiorri, A., Rephaeli, Y., and Shimon, M. (2002). ApJ, 580(2):L101.

Bekestein, J. (1973). ApJ, 183:167.

Bennett, C., Larson, L., Weiland, J., Jarosk, N., Hinshaw, N., Odegard, N., Smith, K., Hill, R.S.; Gold, B., Halpern, M., Komatsu, E., Nolta, M., and Page, L. (2013). ApJS, 208:20. 
Bennett, C. L., Hill, R. S., Hinshaw, G., Nolta, M. R., Odegard, N., Page, L., Spergel, D. N., Weiland, J. L., Wright, E. L., Halpern, M., Jarosik, N., Kogut, A., Limon, M., Meyer, S. S., Tucker, G. S., and Wollack, E. (2003). ApJS, 148:97-117.

Benson, B. A., de Haan, T., Dudley, J. P., Reichardt, C. L., Aird, K. A., Andersson, K., Armstrong, R., Ashby, M. L. N., Bautz, M., Bayliss, M., Bazin, G., Bleem, L. E., Brodwin, M., Carlstrom, J. E., Chang, C. L., Cho, H. M., Clocchiatti, A., Crawford, T. M., Crites, A. T., Desai, S., Dobbs, M. A., Foley, R. J., Forman, W. R., George, E. M., Gladders, M. D., Gonzalez, A. H., Halverson, N. W., Harrington, N., High, F. W., Holder, G. P., Holzapfel, W. L., Hoover, S., Hrubes, J. D., Jones, C., Joy, M., Keisler, R., Knox, L., Lee, A. T., Leitch, E. M., Liu, J., Lueker, M., Luong-Van, D., Mantz, A., Marrone, D. P., McDonald, M., McMahon, J. J., Mehl, J., Meyer, S. S., Mocanu, L., Mohr, J. J., Montroy, T. E., Murray, S. S., Natoli, T., Padin, S., Plagge, T., Pryke, C., Rest, A., Ruel, J., Ruhl, J. E., Saliwanchik, B. R., Saro, A., Sayre, J. T., Schaffer, K. K., Shaw, L., Shirokoff, E., Song, J., Spieler, H. G., Stalder, B., Staniszewski, Z., Stark, A. A., Story, K., Stubbs, C. W., Suhada, R., van Engelen, A., Vanderlinde, K., Vieira, J. D., Vikhlinin, A., Williamson, R., Zahn, O., and Zenteno, A. (2013). ApJ, 763:147.

Bergstrom, L. (2009). New J.Phys., 11:105006.

Berry, C. and Gair, J. (2011). Phys Rev D, 83:104022.

Bertotti, B., Iess, L., and Tortora, P. (2003). Nature, 425:374âĂŞ376.

Bhat, N., Bailes, M., and Verbiest, J. (2008). Phys. Rev. D, 77:124017.

BICEP2 Collaboration (2014). BICEP2 I: Detection Of B-mode Polarization at Degree Angular Scales. Phys. Rev. Lett., 112:241101.

Binney, J. and Tremaine, S. (1994). Galactic Dynamics. Princeton University Press.

Birkinshaw, M. (1999). Physics Reports, 310:97.

Blitz, L., Magnani, L., and Mundy, L. (1984). ApJL, 282:L9.

Bode, P., Ostriker, J., and Vikhlinin, A. (2009). ApJ, 700:989.

Bogdanos, C., Capozziello, S., De Laurentis, M., and Nesseris, S. (2010). Astroparticle Physics, 34:236.

Böhringer, H., Schuecker, P., Guzzo, L., Collins, C., Voges, W., Cruddace, R., OrtizGil, A., Chincarini, G., De Grandi, S., Edge, A., MacGillivray, H., Neumann, D., Schindler, S., and Shaver, P. (2004). A\& A, 425:367-383. 
Böhringer, H., Schuecker, P., Pratt, G., Arnaud, M., Ponman, T., Croston, J., Borgani, S., Bower, R., Briel, U., Collins, C., Donahue, M., Forman, W., Finoguenov, A., Geller, M., Guzzo, L., Henry, J., Kneissl, R., Mohr, J., Matsushita, K., Mullis, C., Ohashi, T., Pedersen, K., Pierini, D., Quintana, H., Raychaudhury, S., Reiprich, T., Romer, A., Rosati, P., Sabirli, K., Temple, R., Viana, P., Vikhlinin, A., Voit, G., and Zhang, Y.-Y. (2007). A\&A, 469:363-377.

Brunetti, G., Venturi, T., Dallacasa, D., Cassano, R., Dolag, K., Giacintucci, S., and Setti, G. (2007). ApJL, 670:L5-L8.

Bryan, G. and Norman, M. (1998). ApJ, 495:80.

Burgay, M., D’Amico, N., Possenti, A., Manchester, R. N., Lyne, A. G., Joshi, B. C., McLaughlin, M. A., Kramer, M., Sarkissian, J. M., Camilo, F., Kalogera, V., Kim, C., and Lorimer, D. R. (2003). Nature, 426:531-533.

Camilo, F., Lorimer, D. R., Freire, P., Lyne, A. G., and Manchester, R. N. (2000). ApJ, 535:975-990.

Capozziello, S., Cardone, V., and Troisi, A. (2007). MNRAS, 375:1423.

Capozziello, S., Corda, C., and de Laurentis, M. (2008). Physics Letters B, 669:255259.

Capozziello, S., De Filippis, E., and Salzano, V. (2009a). MNRAS, 394:947.

Capozziello, S. and De Laurentis, M. (2011). Physics Reports, 509:167.

Capozziello, S., De Laurentis, M., de Martino, I., Formisano, M., and Odintsov, S. (2012). Phys. Rev. D, 85:044022.

Capozziello, S., de Laurentis, M., Nojiri, S., and Odintsov, S. D. (2009b). General Relativity and Gravitation, 41:2313-2344.

Capozziello, S., De Laurentis, M., Odintsov, S., and Stabile, A. (2011). Phys. Rev. D, 83:064004.

Capozziello, S. and Faraoni, V. (2010). Beyond Einstein Gravity: A Survey of gravitational Theories for Cosmology and Astrophysics. Springer, Heidelberg.

Capozziello, S. and Troisi, A. (2005). Phys. Rev. D, 72:044022.

Capozziello, S. and Vignolo, S. (2009). Class. Quant. Grav., 26:175013.

Cardone, V. and Capozziello, S. (2011). MNRAS, 414:1301.

Carroll, B. and Ostlie, D. (2007). An Introduction to Modern Astrophysics. Pearson. 
Cassano, R., Ettori, S., Giacintucci, S., Brunetti, G., Markevitch, M., Venturi, T., and Gitti, M. (2010). ApJL, 721:L82-L85.

Cavaliere, A. and Fusco-Femiano, R. (1976). A\&A, 49:137.

Cavaliere, A. and Fusco-Femiano, R. (1978). A\& A, 70:677.

Chandrasekhar, S. (1931). ApJ, 74:81.

Chang, P. and Hui, L. (2011). ApJ, 732:25.

Choudhury, S., Joshi, G., Mahajan, S., and McKellar, B. (2004). Astroparticle Physics, 21:559.

Clocchiati, A. (2006). ApJ, 642:38.

Cooney, A., De Deo, S., and Psaltis, D. (2010). Phys. Rev. D, 82:064033.

da Silva, A., Barbosa, D., Liddle, A., and Thomas, P. (2000). MNRAS, 317:37.

da Silva, A., Barbosa, D., Liddle, A., and Thomas, P. (2001). MNRAS, 326:155.

Damour, T. and Esposito-Farèse, G. (1992). Class. Quantum Grav, 9:2093.

Damour, T. and Esposito-Farèse, G. (1998). Phys. Rev. D, 58:042001.

Damour, T., Gibbons, G., and Taylor, J. (1988). Phys. Lett., 61:1151.

Damour, T. and Taylor, J. (1991). ApJ, 366:50.

Davis, T. M., Mörtsell, E., Sollerman, J., Becker, A. C., Blondin, S., Challis, P., Clocchiatti, A., Filippenko, A. V., Foley, R. J., Garnavich, P. M., Jha, S., Krisciunas, K., Kirshner, R. P., Leibundgut, B., Li, W., Matheson, T., Miknaitis, G., Pignata, G., Rest, A., Riess, A. G., Schmidt, B. P., Smith, R. C., Spyromilio, J., Stubbs, C. W., Suntzeff, N. B., Tonry, J. L., Wood-Vasey, W. M., and Zenteno, A. (2007). ApJ, 666:716.

De Boni, C., Dolag, K., Ettori, S., Moscardini, L., Pettorino, V., and Baccigalupi, C. (2011). MNRAS, 415:2758-2772.

de Felice, A. and Tsujikawa, S. (2010). $f(R)$ Theories. Living Reviews in Relativity, $13: 3$.

De Laurentis, M. and Capozziello, S. (2011). Astrop. Phys., 35:257.

De Laurentis, M. and De Martino, I. (2013a). MNRAS, 431:741.

De Laurentis, M. and De Martino, I. (2013b). arXiv:1310.0711v2. 
De Martino, I., De Laurentis, M., Atrio-Barandela, F., and Capozziello, S. (2014). MNRAS, 442:921.

Delabrouille, J., Betoule, M., Melin, J.-B., Miville-Deschênes, M.-A., Gonzalez-Nuevo, J., Le Jeune, M., Castex, G., de Zotti, G., Basak, S., Ashdown, M., Aumont, J., Baccigalupi, C., Banday, A. J., Bernard, J.-P., Bouchet, F. R., Clements, D. L., da Silva, A., Dickinson, C., Dodu, F., Dolag, K., Elsner, F., Fauvet, L., Faÿ, G., Giardino, G., Leach, S., Lesgourgues, J., Liguori, M., Macías-Pérez, J. F., Massardi, M., Matarrese, S., Mazzotta, P., Montier, L., Mottet, S., Paladini, R., Partridge, B., Piffaretti, R., Prezeau, G., Prunet, S., Ricciardi, S., Roman, M., Schaefer, B., and Toffolatti, L. (2013). A $\& A$, 553:A96.

Dicke, R., Peebles, P., Roll, P., and Wilkinson, D. (1965). ApJ, 142:414.

Dolag, K., Hansen, F., Roncarelli, M., and Moscardini, L. (2005). MNRAS, 363(1).

Dopita, M. and Sutherland, R. (2003). Astrophysics of the Diffuse Universe. Springer, New York.

Doroshenko, O., Löhmer, O., Kramer, M., Jessner, A., Wielebinski, R., Lyne, A. G., and Lange, C. (2001). A\&A, 379:579-587.

Duffy, A., Schaye, J., Kay, S., and Dalla Vecchia, C. (2008). MNRAS, 390:L64.

Dvali, G., Gabadadze, G., and Porrati, M. (2000). Phys. Lett. B, 485:208-214.

Ebeling, H., Edge, A., Allen, S., Crawford, C., Fabian, A., and Huchra, J. (2000). MNRAS, 318:333.

Ebeling, H., Edge, A., Böhringer, H., Allen, S., Crawford, C., Fabian, A., Voges, W., and Huchra, J. (1998). MNRAS, 301:881.

Ebeling, H., Mullis, C., and Tully, R. (2002). ApJ, 580:774.

Eddington, A. (1924). The mathematical theory of relativity,. Cambridge University Press London.

Einstein, A. (1905a). Annalen der Physik, 17:891-921.

Einstein, A. (1905b). Annalen der Physik, 18:639-641.

Einstein, A. (1916). Annalen der Physik, 49:769.

Fabbri, R., Melchiorri, F., and Natale, V. (1978). Astrophys. Space Sci., 59:223.

Falle, S. (2007). Numerical Simulations of Star Formation, page 3.

Faraoni, V. (2005). Class. Quantum Grav., 22:32352. 
Faraoni, V. and Gunzig, E. (1999). Int.J.Theor.Phys., 38:217-225.

Ferraro, S., Schimdt, F., and Hu, W. (2011). Phys Rev D, 83:063503.

Finn, L. and Sutton, P. (2002). Phys Rev D, 62:044022.

Fixsen, D. J., Cheng, E. S., Gales, J. M., Mather, J. C., Shafer, R. A., and Wright, E. L. (1996). ApJ, 473:576.

Fomalont, E. and Kopeikin, S. (2003). ApJ, 598:704-711.

Fomalont, E., Kopeikin, S., Jones, D., Honma, M., and Titov, O. (2010). Proceedings of the IAU Symposium, 261:291-295.

Freese, K., Adams, F. C., Frieman, J. A., and Mottola, E. (1987). Nuclear Physics B, 287:797.

Freire, P. C., Camilo, F., Kramer, M., Lorimer, D. R., Lyne, A. G., Manchester, R. N., and D'Amico, N. (2003). MNRAS, 340:1359-1374.

Freire, P. C. C., Wex, N., Esposito-Farèse, G., Verbiest, J. P. W., Bailes, M., Jacoby, B. A., Kramer, M., Stairs, I. H., Antoniadis, J., and Janssen, G. H. (2012). MNRAS, 423:3328-3343.

Fruchter, A., Stinebring, D., and Taylor, J. (1988). Nature, 333:237-239.

Fusco-Femiano, R., Lapi, A., and Cavaliere, A. (2013). ApJL, 763:L3.

Goldhaber, A. and Nieto, M. (1974). Mass of the graviton. Phys. Rev. D, 9:1119-1121.

Górski, K., Hivon, E., Banday, A., Wandelt, B., Hansen, F., Reinecke, M., and Bartelmann, M. (2005). ApJ, 622(2):759.

Guth, A. (1981). Phys. Rev. D, 23(347).

Hamuy, M., Phillips, M. M., Suntzeff, N. B., Schommer, R. A., Maza, J., and Aviles, R. (1996). Astron. J., 112:2391.

Hand, N., Appel, J. W., Battaglia, N., Bond, J. R., Das, S., Devlin, M. J., Dunkley, J., Dünner, R., Essinger-Hileman, T., Fowler, J. W., Hajian, A., Halpern, M., Hasselfield, M., Hilton, M., Hincks, A. D., Hlozek, R., Hughes, J. P., Irwin, K. D., Klein, J., Kosowsky, A., Lin, Y.-T., Marriage, T. A., Marsden, D., McLaren, M., Menanteau, F., Moodley, K., Niemack, M. D., Nolta, M. R., Page, L. A., Parker, L., Partridge, B., Plimpton, R., Reese, E. D., Rojas, F., Sehgal, N., Sherwin, B. D., Sievers, J. L., Spergel, D. N., Staggs, S. T., Swetz, D. S., Switzer, E. R., Thornton, R., Trac, H., Visnjic, K., and Wollack, E. (2011). ApJ, 736:39. 
Harrison, E. (1970). Phys. Rev. D, 1:2726.

Hasselfield, M., Hilton, M., Marriage, T. A., Addison, G. E., Barrientos, L. F., Battaglia, N., Battistelli, E. S., Bond, J. R., Crichton, D., Das, S., Devlin, M. J., Dicker, S. R., Dunkley, J., Dünner, R., Fowler, J. W., Gralla, M. B., Hajian, A., Halpern, M., Hincks, A. D., Hlozek, R., Hughes, J. P., Infante, L., Irwin, K. D., Kosowsky, A., Marsden, D., Menanteau, F., Moodley, K., Niemack, M. D., Nolta, M. R., Page, L. A., Partridge, B., Reese, E. D., Schmitt, B. L., Sehgal, N., Sherwin, B. D., Sievers, J., Sifón, C., Spergel, D. N., Staggs, S. T., Swetz, D. S., Switzer, E. R., Thornton, R., Trac, H., and Wollack, E. J. (2013). JCAP, 07:008H.

Hinshaw, G., Larson, D., Komatsu, E., Spergel, D. N., Bennett, C. L., Dunkley, J., Nolta, M. R., Halpern, M., Hill, R. S., Odegard, N., Page, L., Smith, K. M., Weiland, J. L., Gold, B., Jarosik, N., Kogut, A., Limon, M., Meyer, S. S., Tucker, G. S., Wollack, E., and Wright, E. L. (2013). ApJS, 208:19.

Hinterbichler, K. and Khoury , J. (2010). Phys. Rev. Lett., 104:231301.

Hofmann, F., Müller, J., and Biskupek, L. (2010). A\&A, 522:L5.

Hoskin, M. (1999). The Cambridge concise history of astronomy. Cambridge University Press.

Hu, W. and Sawicki, I. (2007). Phys. Rev. D, 76:064004.

Hulse, R. and Taylor, J. (1975). ApJ, 195:L51.

Hurier, G., Aghanim, N., Douspis, M., and Pointecouteau, E. (2014). A\&SA, 561:A143.

Hurier, G., Macías-Pérez, J. F., and Hildebrandt, S. (2013). AESA, 558:A118.

Itoh, N., Kohyama, Y., and Nozawa, S. (1998). ApJ, 502:7.

Jacoby, B. A., Bailes, M., van Kerkwijk, M. H., Ord, S., Hotan, A., Kulkarni, S. R., and Anderson, S. B. (2003). ApJL, 599:L99-L102.

Jacoby, B. A., Cameron, P. B., Jenet, F. A., Anderson, S. B., Murty, R. N., and Kulkarni, S. R. (2006). ApJL, 644:L113-L116.

Jaeckel, J. and Ringwald, A. (2010). Annual Review of Nuclear and Particle Science, 60:405.

Janssen, G. H., Stappers, B. W., Kramer, M., Nice, D. J., Jessner, A., Cognard, I., and Purver, M. B. (2008). A\&A, 490:753-761.

Jetzer, P., Puy, D., Signore, M., and Tortora, C. (2011). General Relativity and Gravitation, 43:1083-1093. 
Jimenez, R., Verde, L., Treu, T., and Stern, D. (2003). ApJ, 593:622-629.

Johnston, S., Lorimer, D. R., Harrison, P. A., Bailes, M., Lyne, A. G., Bell, J. F., Kaspi, V. M., Manchester, R. N., D'Amico, N., and Nicastro, L. (1993). Nature, 361:613-615.

Jones, C. and Forman, W. (1984). ApJ, 276:38.

Kashlinsky, A., Atrio-Barandela, F., Kocevski, D., and Ebeling, H. (2009). ApJ, 691:1479.

Kaspi, V., Bailes, M., Manchester, R., Stappers, B., and Bell, J. (1996). Nature, 381:584-586.

Kaspi, V. M., Lyne, A. G., Manchester, R. N., Crawford, F., Camilo, F., Bell, J. F., D'Amico, N., Stairs, I. H., McKay, N. P. F., Morris, D. J., and Possenti, A. (2000). ApJ, 543:321-327.

Kazanas, D. (1980). ApJL, 241:L59-L63.

Khoury , J. and Weltman, A. (2004). Physical Review Letters, 93:171104.

Khoury, J. and Wyman, M. (2009). Phys Rev D, 80:064023.

Kippenhahn, R. and Weigert, A. (1990). Stellar structures and evolution. SpringerVerlag.

Klessen, R. S., Krumholz, M. R., and Heitsch, F. (2009). ArXiv e-prints.

Kobayashi, T. and Maeda, K. (2008). Phys Rev D, 78:064019.

Kocevski, D. and Ebeling, H. (2006). ApJ, 645:1043.

Kocevski, D., Ebeling, H., Mullis, C., and Tully, R. (2007). ApJ, 662:224.

Komatsu, E. and Seljak, U. (2001). MNRAS, 327:1353-1366.

Komatsu, E., Smith, K. M., Dunkley, J., Bennett, C. L., Gold, B., Hinshaw, G., Jarosik, N., Larson, D., Nolta, M. R., Page, L., Spergel, D. N., Halpern, M., Hill, R. S., Kogut, A., Limon, M., Meyer, S. S., Odegard, N., Tucker, G. S., Weiland, J. L., Wollack, E., and Wright, E. L. (2011). ApJ, 192:18.

Konacki, M., Wolszczan, A., and Stairs, I. H. (2003). ApJ, 589:495-502.

Kopeikin, S. and Fomalont, E. (2007). General Relativity and Gravitation, 39:15831624. 
Kowalski, M., Rubin, D., Aldering, G., Agostinho, R. J., Amadon, A., Amanullah, R., Balland, C., Barbary, K., Blanc, G., Challis, P. J., Conley, A., Connolly, N. V., Covarrubias, R., Dawson, K. S., Deustua, S. E., Ellis, R., Fabbro, S., Fadeyev, V., Fan, X., Farris, B., Folatelli, G., Frye, B. L., Garavini, G., Gates, E. L., Germany, L., Goldhaber, G., Goldman, B., Goobar, A., Groom, D. E., Haissinski, J., Hardin, D., Hook, I., Kent, S., Kim, A. G., Knop, R. A., Lidman, C., Linder, E. V., Mendez, J., Meyers, J., Miller, G. J., Moniez, M., Mourão, A. M., Newberg, H., Nobili, S., Nugent, P. E., Pain, R., Perdereau, O., Perlmutter, S., Phillips, M. M., Prasad, V., Quimby, R., Regnault, N., Rich, J., Rubenstein, E. P., Ruiz-Lapuente, P., Santos, F. D., Schaefer, B. E., Schommer, R. A., Smith, R. C., Soderberg, A. M., Spadafora, A. L., Strolger, L.-G., Strovink, M., Suntzeff, N. B., Suzuki, N., Thomas, R. C., Walton, N. A., Wang, L., Wood-Vasey, W. M., Yun, J. L., and Supernova Cosmology Project (2008). ApJ, 686:749.

Kramer, M., Stairs, I. H., Manchester, R. N., McLaughlin, M. A., Lyne, A. G., Ferdman, R. D., Burgay, M., Lorimer, D. R., Possenti, A., D'Amico, N., Sarkissian, J. M., Hobbs, G. B., Reynolds, J. E., Freire, P. C. C., and Camilo, F. (2006). Science, 314:97-102.

Kravtsov, A. V. and Borgani, S. (2012). ARAA, 50:353.

Lada, C. and Kylafis, N. (1999). The Origin of Stars and Planetary Systems. ed. Springer.

Lanczos, C., Z. (1931). Phys, 73:147.

Landau, L. and Lifshitz, E. (1962). The Classical Theory of Fields. Addison-Wesley Pub.Co., Inc., Reading.

Le Verrier, U. (1859). Académie des sciences (Paris), 49:379-383.

Li, B. and Barrow, J. (2007). Phys. Rev. D, 75:084010.

Lima, J., Silva, A. I., and Viegas, S. M. (2000). MNRAS, 312:747-752.

Lima, J. and Trodden, M. (1996). Phys. Rev. D, 53:4280.

LoSecco, J., Mathews, G., and Wang, Y. (2001). Phys.Rev. D, 64:123002.

Lu, L., Sargent, W. L. W., Barlow, T. A., Churchill, C. W., and Vogt, S. S. (1996). ApJS, 107:475.

Lubin, L. M., Cen, R., Bahcall, N. A., and Ostriker, J. P. (1996). ApJ, 460:10.

Lundgren, S., Zepka, A., and Cordes, J. (1995). ApJ, 453:419-423. 
Luzzi, G., Shimon, M., Lamagna, L., Rephaeli, Y., De Petris, M., Conte, A., De Gregori, S., and Battistelli, E. (2009). ApJ, 705:1122-1128.

Lynch, R., Freire, P., Ransom, S., and Jacoby, B. (2012). ApJ, 745:109.

Lyne, A. G., Biggs, J. D., Brinklow, A., McKenna, J., and Ashworth, M. (1988). Nature, 332:45-47.

Maggiore, M. (2007). Gravitational Waves: Theory and Experiments. Oxford University Press, USA.

Magnano, G. and Sokolowski, L. (1994). Phys. Rev. D, 50:5039.

Mather, J., Fixsen, D., Shafer, R., Mosier, C., and Wilkinson, D. (1999). ApJ, 512:511.

Matyjasek (1995). Phys.Rev. D, 51:4154.

McConnell, D., McCulloch, P. M., Hamilton, P. A., Ables, J. G., Hall, P. J., Jacka, C. E., and Hunt, A. J. (1991). MNRAS, 249:654-657.

McKee, C. and Ostriker, E. (2007). Annu. Rev. Astron. Astrophys., 45:565.

Menanteau, F., Sifón, C., Barrientos, L. F., Battaglia, N., Bond, J. R., Crichton, D., Das, S., Devlin, M. J., Dicker, S., Dünner, R., Gralla, M., Hajian, A., Hasselfield, M., Hilton, M., Hincks, A. D., Hughes, J. P., Infante, L., Kosowsky, A., Marriage, T. A., Marsden, D., Moodley, K., Niemack, M. D., Nolta, M. R., Page, L. A., Partridge, B., Reese, E. D., Schmitt, B. L., Sievers, J., Spergel, D. N., Staggs, S. T., Switzer, E., and Wollack, E. J. (2013). ApJ, 765:67.

Meyer, D. M., York, D. G., Black, J. H., Chaffee, J. F. H., and Foltz, C. B. (1986). ApJL, 308:L37.

Miranda, V., Jorás, S. E., Waga, I., and Quartin, M. (2009). Physical Review Letters, 102(22):221101.

Moresco, M., Cimatti, A., Jimenez, R., Pozzetti, L., Zamorani, G., Bolzonella, M., Dunlop, J., Lamareille, F., Mignoli, M., Pearce, H., Rosati, P., Stern, D., Verde, L., Zucca, E., Carollo, C. M., Contini, T., Kneib, J.-P., Le Fèvre, O., Lilly, S. J., Mainieri, V., Renzini, A., Scodeggio, M., Balestra, I., Gobat, R., McLure, R., Bardelli, S., Bongiorno, A., Caputi, K., Cucciati, O., de la Torre, S., de Ravel, L., Franzetti, P., Garilli, B., Iovino, A., Kampczyk, P., Knobel, C., Kovač, K., Le Borgne, J.-F., Le Brun, V., Maier, C., Pelló, R., Peng, Y., Perez-Montero, E., Presotto, V., Silverman, J. D., Tanaka, M., Tasca, L. A. M., Tresse, L., Vergani, D., Almaini, O., Barnes, L., Bordoloi, R., Bradshaw, E., Cappi, A., Chuter, R., Cirasuolo, M., Coppa, G., Diener, C., Foucaud, S., Hartley, W., Kamionkowski, 
M., Koekemoer, A. M., López-Sanjuan, C., McCracken, H. J., Nair, P., Oesch, P., Stanford, A., and Welikala, N. (2012a). JCAP, 08:006.

Moresco, M., Verde, L., Pozzetti, L., Jimenez, R., and Cimatti, A. (2012b). JCAP, 07:053.

Mortonson, M., Hu, W., and Huterer, D. (2009). Phys.Rev. D, 80:067301.

Mortonson, M. J. and Seljak, U. (2014). A joint analysis of Planck and BICEP2 B modes including dust polarization uncertainty. ArXiv e-prints.

Murphy, M. T., Webb, J. K., and Flambaum, V. V. (2003). MNRAS, 345:609.

Napolitano, N. R., Capozziello, S., Romanowsky, A. J., Capaccioli, M., and Tortora, C. (2012). ApJ, 748:87.

Navarro, J., Frenk, C., and White, S. (1997). ApJ, 490:493.

Nice, D., Sayer, R., and Taylor, J. (1996). ApJ, L87-L90:466.

Nice, D., Splaver, E., and Stairs, I. H. (2001). ApJ, 516-521:549.

Nice, D., Taylor, J., and Fruchter, A. S. (1993). ApJ, L49-L52:402.

Nice, D. and Thorsett, S. (2003). ASP Conf. Series, 302:93.

Nice, D. J., Stairs, I. H., and Kasian, L. E. (2008). Masses of Neutron Stars in Binary Pulsar Systems. In Bassa, C., Wang, Z., Cumming, A., and Kaspi, V. M., editors, 40 Years of Pulsars: Millisecond Pulsars, Magnetars and More, volume 983 of American Institute of Physics Conference Series, pages 453-458.

Nicolis, A., Rattazzi, R., and Trincherini, E. (2009). Phys. Rev. D, 79:064036.

Nojiri, S., Odintsov, S., and Tsujikawa, S. (2005). Phys.Rev. D, 71:063004.

Nordtvedt, K. (1990). Phis. Review. Letter, 65:95.

Noterdaeme, P., Petitjean, P., Srianand, R., Ledoux, C., and López, S. (2011). A\&A, 526:L7.

Nozawa, S., Itoh, N., and Kohyama, Y. (1998). ApJ, 508:17.

Nunes, N., Dent, T., Martins, C., and Robbers, G. (2009). Mem. Soc. Ast. It., 80:785.

Olmo, G. (2011). Int. J. Mod. Phys. D, 20:413.

Overduin, J. and Cooperstock, F. (1998). Phys.Rev. D, 58:043506.

Pauli, W. (1919). Phys. Zeit., 20:457. 
Peacock, J. (1999). Cosmological Physics. Cambridge University Press.

Peebles, J. (1980). The Large-Scale Structure of the Universe. Princeton University Press.

Peebles, P. J. and Ratra, B. (2003). Reviews of Modern Physics, 75:559-606.

Percival, W. J., Baugh, C. M., Bland-Hawthorn, J., Bridges, T., Cannon, R., Cole, S., Colless, M., Collins, C., Couch, W., Dalton, G., De Propris, R., Driver, S. P., Efstathiou, G., Ellis, R. S., Frenk, C. S., Glazebrook, K., Jackson, C., Lahav, O., Lewis, I., Lumsden, S., Maddox, S., Moody, S., Norberg, P., Peacock, J. A., Peterson, B. A., Sutherland, W., and Taylor, K. (2001). MNRAS, 327:1297.

Perlmutter, S., Aldering, G., Goldhaber, G., Knop, R. A., Nugent, P., Castro, P. G., Deustua, S., Fabbro, S., Goobar, A., Groom, D. E., Hook, I. M., Kim, A. G., Kim, M. Y., Lee, J. C., Nunes, N. J., Pain, R., Pennypacker, C. R., Quimby, R., Lidman, C., Ellis, R. S., Irwin, M., McMahon, R. G., Ruiz-Lapuente, P., Walton, N., Schaefer, B., Boyle, B. J., Filippenko, A. V., Matheson, T., Fruchter, A. S., Panagia, N., Newberg, H. J. M., Couch, W. J., and Project, T. S. C. (1999). ApJ, 517:565-586.

Perlmutter, S., Gabi, S., Goldhaber, G., Goobar, A., Groom, D. E., Hook, I. M., Kim, A. G., Kim, M. Y., Lee, J. C., Pain, R., Pennypacker, C. R., Small, I. A., Ellis, R. S., McMahon, R. G., Boyle, B. J., Bunclark, P. S., Carter, D., Irwin, M. J., Glazebrook, K., Newberg, H. J. M., Filippenko, A. V., Matheson, T., Dopita, M., and Couch, W. J. (1997). ApJ, 483:565-581.

Peter, A. (2012). arXiv: astro-ph/1201.3942.

Planck Collaboration (2011a). Planck early results. VII. The Early Release Compact Source Catalogue. A $\because A, 536:$ A7.

Planck Collaboration (2011b). Planck early results. XI. Calibration of the local galaxy cluster Sunyaev-Zeldovich scaling relations. $A \mathscr{\xi} A, 536$ :A11.

Planck Collaboration (2012). Planck intermediate results. V: Pressure profiles of galaxy clusters from the Sunyaev-Zeldovich effect. A\&A, 550:A131.

Planck Collaboration (2013a). Planck 2013 results. I: Overview of products and results. A\&A, accepted. ArXiv:1303.5062.

Planck Collaboration (2013b). Planck 2013 results. II: Low Frequency Instrument data processing. A\&A, accepted. ArXiv:1303.5063. 
Planck Collaboration (2013c). Planck 2013 results. III: LFI systematic uncertainties. $A \& \mathcal{E}$, accepted. ArXiv:1303.5064.

Planck Collaboration (2013d). Planck 2013 results. VII: HFI time response and beams. $A \mathscr{E} A$, accepted. ArXiv:1303.5068.

Planck Collaboration (2013e). Planck 2013 results. XI. All-sky model of thermal dust emission. $A \& A$.

Planck Collaboration (2013f). Planck 2013 results. XII: Component separation. A\& $A$, accepted. ArXiv:1303.5068.

Planck Collaboration (2013g). Planck 2013 results. XIII: Galactic CO emission. A $\& A$, accepted. ArXiv:1303.5069.

Planck Collaboration (2013h). Planck 2013 results. XIX: The integrated Sachs-Wolfe effect. $A \& A$, accepted. ArXiv:1303.5079.

Planck Collaboration (2013i). Planck 2013 results. XV: CMB power spectra and likelihood. $A \mathscr{E} A$, accepted. ArXiv:1303.5075.

Planck Collaboration (2013j). Planck 2013 results. XVI: Cosmological parameters. $A \mathscr{E} A$, accepted. ArXiv:1303.5076.

Planck Collaboration (2013k). Planck 2013 results. XVIII: The gravitational lensinginfrared background correlation. A\&SA, accepted. ArXiv:1303.5078.

Planck Collaboration (20131). Planck 2013 results. XX: Cosmology from SunyaevZeldovich cluster counts. AEA, accepted. ArXiv:1303.5080.

Planck Collaboration (2013m). Planck 2013 results. XXI: Cosmology with the all-sky Planck Compton parameter y-map. A\&AA, accepted. ArXiv:1303.5081.

Planck Collaboration (2013n). Planck 2013 results. XXIII: Isotropy and statistics of the CMB. A\&SA, accepted. ArXiv:1303.5083.

Planck Collaboration (2013o). Planck 2013 results. XXIV: Constraints on primordial non-Gaussianity. AESA, accepted. ArXiv:1303.5084.

Planck Collaboration (2013p). Planck 2013 results. XXIX: Planck catalogue of Sunyaev-Zeldovich sources. A\&A, accepted. ArXiv:1303.5089.

Planck Collaboration (2013q). Planck 2013 results. XXVIII. The Planck Catalogue of Compact Sources. ArXiv e-prints.

Planck Collaboration (2013r). Planck intermediate results. X: Physics of the hot gas in the Coma cluster. $A \& A, 554: 140$. 
Pope, A. C., Matsubara, T., Szalay, A. S., Blanton, M. R., Eisenstein, D. J., Gray, J., Jain, B., Bahcall, N. A., Brinkmann, J., Budavari, T., Connolly, A. J., Frieman, J. A., Gunn, J. E., Johnston, D., Kent, S. M., Lupton, R. H., Meiksin, A., Nichol, R. C., Schneider, D. P., Scranton, R., Strauss, M. A., Szapudi, I., Tegmark, M., Vogeley, M. S., Weinberg, D. H., Zehavi, I., and SDSS Collaboration (2004). ApJ, 607:655.

Possenti, A., D'Amico, N., Manchester, R. N., Camilo, F., Lyne, A. G., Sarkissian, J., and Corongiu, A. (2003). ApJ, 599:475-484.

Puy, D. (2004). A\&A, 422:1-9.

Quandt, I. and Schmidt, H. (1991). Astron. Nachr., 312:97.

Rephaeli, Y. (1980). ApJ, 241:858.

Riess, A. G., Filippenko, A. V., Challis, P., Clocchiatti, A., Diercks, A., Garnavich, P. M., Gilliland, R. L., Hogan, C. J., Jha, S., Kirshner, R. P., Leibundgut, B., Phillips, M. M., Reiss, D., Schmidt, B. P., Schommer, R. A., Smith, R. C., Spyromilio, J., Stubbs, C., Suntzeff, N. B., and Tonry, J. (1998). Astron. J, 116:10091038.

Riess, A. G., Strolger, L.-G., Tonry, J., Casertano, S., Ferguson, H. C., Mobasher, B., Challis, P., Filippenko, A. V., Jha, S., Li, W., Chornock, R., Kirshner, R. P., Leibundgut, B., Dickinson, M., Livio, M., Giavalisco, M., Steidel, C. C., Benítez, T., and Tsvetanov, Z. (2004). ApJ, 607:665-687.

Roman-Duval, J., Jackson, J., Heyer, M., Rathborne, H., and Simon, R. (2010). ApJ, 723:492.

Roth, K. C. and Bauer, J. M. (1999). ApJL, 515:L57.

Rubano, C. and Scudellaro, P. (2005). Gen. Relat. Gravit., 37:521.

Sanders, R. (1984). A\&A, 136:L21.

Sarazin, C. L. (1986). X-ray emission from clusters of galaxies. Rev. Mod. Phys., 58:1-115.

Sayers, J., Czakon, N. G., Mantz, A., Golwala, S. R., Ameglio, S., Downes, T. P., Koch, P. M., Lin, K.-Y., Maughan, B. J., Molnar, S. M., Moustakas, L., Mroczkowski, T., Pierpaoli, E., Shitanishi, J. A., Siegel, S., Umetsu, K., and Van der Pyl, N. (2013). ApJ, 768(2):177.

Scheffler, H. and Elsasser, H. (1982). Physics of the Galaxy and Interstellar Matter. Springer, New York. 
Schimdt, F., Vikhlinin, A., and Hu, W. (2009). Phys. Rev. D, 80:083505.

Sehgal, N., Trac, H., Acquaviva, V., Ade, P. A. R., Aguirre, P., Amiri, M., Appel, J. W., Barrientos, L. F., Battistelli, E. S., Bond, J. R., Brown, B., Burger, B., Chervenak, J., Das, S., Devlin, M. J., Dicker, S. R., Bertrand Doriese, W., Dunkley, J., Dünner, R., Essinger-Hileman, T., Fisher, R. P., Fowler, J. W., Hajian, A., Halpern, M., Hasselfield, M., Hernández-Monteagudo, C., Hilton, G. C., Hilton, M., Hincks, A. D., Hlozek, R., Holtz, D., Huffenberger, K. M., Hughes, D. H., Hughes, J. P., Infante, L., Irwin, K. D., Jones, A., Baptiste Juin, J., Klein, J., Kosowsky, A., Lau, J. M., Limon, M., Lin, Y.-T., Lupton, R. H., Marriage, T. A., Marsden, D., Martocci, K., Mauskopf, P., Menanteau, F., Moodley, K., Moseley, H., Netterfield, C. B., Niemack, M. D., Nolta, M. R., Page, L. A., Parker, L., Partridge, B., Reid, B., Sherwin, B. D., Sievers, J., Spergel, D. N., Staggs, S. T., Swetz, D. S., Switzer, E. R., Thornton, R., Tucker, C., Warne, R., Wollack, E., and Zhao, Y. (2011). ApJ, $732: 44$.

Seljak, U. (2000). MNRAS, 318:203.

Shi, X. and Komatsu, E. (2014). MNRAS, 442:521.

Shklovskii, I. (1973). Soviet Astronomy, 13:562.

Simon, J., Verde, L., and Jimenez, R. (2005). Phis. Rev. D, 71(12).

Smith, K., Dvorkin, C., Boyle, L., Turok, N., Halpern, M., Hinshaw, G., and Gold, B. (2014). Phys. Rev. Lett., 113:031301.

Songaila, A., Cowie, L., Hogan, C., and Rugers, M. (1994a). Nature, 368:599.

Songaila, A., Cowie, L. L., Vogt, S., Keane, M., Wolfei, A. M., Hu, E. M., Oren, A. L., Tytleri, D.-R., and Lanzetta, K. M. (1994b). Nature, 371:43.

Srianand, R., Chand, H., Petitjean, P., and Aracil, B. (2004). Phys. Rev. Lett., 92:121302.

Srianand, R., Petitjean, P., and Ledoux, C. (2000). Nature, 408:931.

Stairs, I. H. (2004). Science, 304:547.

Stairs, I. H., Thorsett, S. E., Taylor, J. H., and Wolszczan, A. (2002). ApJ, 581:501.

Staniszewski, Z., Ade, P. A. R., Aird, K. A., Benson, B. A., Bleem, L. E., Carlstrom, J. E., Chang, C. L., Cho, H.-M., Crawford, T. M., Crites, A. T., de Haan, T., Dobbs, M. A., Halverson, N. W., Holder, G. P., Holzapfel, W. L., Hrubes, J. D., Joy, M., Keisler, R., Lanting, T. M., Lee, A. T., Leitch, E. M., Loehr, A., Lueker, 
M., McMahon, J. J., Mehl, J., Meyer, S. S., Mohr, J. J., Montroy, T. E., Ngeow, C.-C., Padin, S., Plagge, T., Pryke, C., Reichardt, C. L., Ruhl, J. E., Schaffer, K. K., Shaw, L., Shirokoff, E., Spieler, H. G., Stalder, B., Stark, A. A., Vanderlinde, K., Vieira, J. D., Zahn, O., and Zenteno, A. (2009). ApJ, 701:32.

Stappers, B. W., Bailes, M., Lyne, A. G., Manchester, R. N., D’Amico, N., Tauris, T. M., Lorimer, D. R., Johnston, S., and Sandhu, J. S. (1996). ApJL, 465:L119.

Starobinsky, A. (1980). Phys. Lett. B, 91:99.

Starobinsky, A. (2007). JETP Lett., 86:157.

Steigman, G. (2006). Int.J.Mod.Phys, E15:1-36.

Stelle, K. (1978). Gen. Relat. Gravit., 9:343.

Stern, D., Jimenez, R., Verde, L., Kamionkowski, M., and Stanford, S. (2010). JCAP, $2: 8$.

Sunyaev, R. and Zeldovich, Y. (1972). Comments on Astrophys. Space Phys, 4:173.

Sunyaev, R. and Zeldovich, Y. (1980). MNRAS, 190:413.

Suzuki, N., Rubin, D., Lidman, C., Aldering, G., Amanullah, R., Barbary, K., Barrientos, L. F., Botyanszki, J., Brodwin, M., Connolly, N., Dawson, K. S., Dey, A., Doi, M., Donahue, M., Deustua, S., Eisenhardt, P., Ellingson, E., Faccioli, L., Fadeyev, V., Fakhouri, H. K., Fruchter, A. S., Gilbank, D. G., Gladders, M. D., Goldhaber, G., Gonzalez, A. H., Goobar, A., Gude, A., Hattori, T., Hoekstra, H., Hsiao, E., Huang, X., Ihara, Y., Jee, M. J., Johnston, D., Kashikawa, N., Koester, B., Konishi, K., Kowalski, M., Linder, E. V., Lubin, L., Melbourne, J., Meyers, J., Morokuma, T., Munshi, F., Mullis, C., Oda, T., Panagia, N., Perlmutter, S., Postman, M., Pritchard, T., Rhodes, J., Ripoche, P., Rosati, P., Schlegel, D. J., Spadafora, A., Stanford, S. A., Stanishev, V., Stern, D., Strovink, M., Takanashi, N., Tokita, K., Wagner, M., Wang, L., Yasuda, N., Yee, H. K. C., and Supernova Cosmology Project, T. (2012). ApJ, 746:85.

Tegmark, M., Blanton, M. R., Strauss, M. A., Hoyle, F., Schlegel, D., Scoccimarro, R., Vogeley, M. S., Weinberg, D. H., Zehavi, I., Berlind, A., Budavari, T., Connolly, A., Eisenstein, D. J., Finkbeiner, D., Frieman, J. A., Gunn, J. E., Hamilton, A. J. S., Hui, L., Jain, B., Johnston, D., Kent, S., Lin, H., Nakajima, R., Nichol, R. C., Ostriker, J. P., Pope, A., Scranton, R., Seljak, U., Sheth, R. K., Stebbins, A., Szalay, A. S., Szapudi, I., Verde, L., Xu, Y., Annis, J., Bahcall, N. A., Brinkmann, J., Burles, S., Castander, F. J., Csabai, I., Loveday, J., Doi, M., Fukugita, M., Gott, III, J. R., Hennessy, G., Hogg, D. W., Ivezić, Ž., Knapp, G. R., Lamb, D. Q., 
Lee, B. C., Lupton, R. H., McKay, T. A., Kunszt, P., Munn, J. A., O'Connell, L., Peoples, J., Pier, J. R., Richmond, M., Rockosi, C., Schneider, D. P., Stoughton, C., Tucker, D. L., Vanden Berk, D. E., Yanny, B., York, D. G., and SDSS Collaboration (2004). ApJ, 606:702.

Tegmark, M., de Oliveira-Costa, A., and Hamilton, A. J. (2003). Phys. Rev. D, 68(12):123523.

Thorsett, S., Arzoumanian, Z., Camilo, F., and Lyne, A. G. (1999). ApJ, 523:763-770.

Tsujikawa, S. (2011). Dark Energy: Investigation and Modeling, volume 370 of Astrophysics and Space Science Library. Springer Netherlands.

Upadhye, A., Hu, W., and Mellier, Y. (2010). Phys.Rev. D, 82:064002.

Uzan, J. (2003). Reviews of Modern Physics, 75:403-455.

Vanderlinde, K., Crawford, T. M., de Haan, T., Dudley, J. P., Shaw, L., Ade, P. A. R., Aird, K. A., Benson, B. A., Bleem, L. E., Brodwin, M., Carlstrom, J. E., Chang, C. L., Crites, A. T., Desai, S., Dobbs, M. A., Foley, R. J., George, E. M., Gladders, M. D., Hall, N. R., Halverson, N. W., High, F. W., Holder, G. P., Holzapfel, W. L., Hrubes, J. D., Joy, M., Keisler, R., Knox, L., Lee, A. T., Leitch, E. M., Loehr, A., Lueker, M., Marrone, D. P., McMahon, J. J., Mehl, J., Meyer, S. S., Mohr, J. J., Montroy, T. E., Ngeow, C.-C., Padin, S., Plagge, T., Pryke, C., Reichardt, C. L., Rest, A., Ruel, J., Ruhl, J. E., Schaffer, K. K., Shirokoff, E., Song, J., Spieler, H. G., Stalder, B., Staniszewski, Z., Stark, A. A., Stubbs, C. W., van Engelen, A., Vieira, J. D., Williamson, R., Yang, Y., Zahn, O., and Zenteno, A. (2010). ApJ, 722:1180.

Verbiest, J. P. W., Bailes, M., Coles, W. A., Hobbs, G. B., van Straten, W., Champion, D. J., Jenet, F. A., Manchester, R. N., Bhat, N. D. R., Sarkissian, J. M., Yardley, D., Burke-Spolaor, S., Hotan, A. W., and You, X. P. (2009). MNRAS, 400:951-968.

Verbiest, J. P. W., Bailes, M., van Straten, W., Hobbs, G. B., Edwards, R. T., Manchester, R. N., Bhat, N. D. R., Sarkissian, J. M., Jacoby, B. A., and Kulkarni, S. R. (2008). ApJ, 679:675-680.

Wald, R. (1984). General Relativity. University Of Chicago Press.

Weinberg, S. (1972). Gravitation and Cosmology. New York:Wiley.

Weisberg, J., Nice, D. J., and Taylor, J. H. (2010). ApJ, 722:1030-1034.

Weisberg, J. M. and Taylor, J. H. (2002). Radio Pulsars. M. Bailes.

Weyl, H. (1918). Math. Zeit., 2:384. 
White, D. A. and Fabian, A. C. (1995). MNRAS, 273:72-84.

White, D.A., J. C. and Forman, W. (1997). ApJ, 292:419.

White, S. D. M., Navarro, J. F., Evrard, A. E., and Frenk, C. S. (1993). Nature, 366:429-433.

Will, C. (2006). Living Rev. Relativity, 9:3.

Will, C. M. (1998). Phys. Rev. D, 57:2061-2068.

Williams, J., Turyshev, S., and Boggs, D. (2004). Physical Review Letters, 93:10171025.

Williamson, R., Benson, B. A., High, F. W., Vanderlinde, K., Ade, P. A. R., Aird, K. A., Andersson, K., Armstrong, R., Ashby, M. L. N., Bautz, M., Bazin, G., Bertin, E., Bleem, L. E., Bonamente, M., Brodwin, M., Carlstrom, J. E., Chang, C. L., Chapman, S. C., Clocchiatti, A., Crawford, T. M., Crites, A. T., de Haan, T., Desai, S., Dobbs, M. A., Dudley, J. P., Fazio, G. G., Foley, R. J., Forman, W. R., Garmire, G., George, E. M., Gladders, M. D., Gonzalez, A. H., Halverson, N. W., Holder, G. P., Holzapfel, W. L., Hoover, S., Hrubes, J. D., Jones, C., Joy, M., Keisler, R., Knox, L., Lee, A. T., Leitch, E. M., Lueker, M., Luong-Van, D., Marrone, D. P., McMahon, J. J., Mehl, J., Meyer, S. S., Mohr, J. J., Montroy, T. E., Murray, S. S., Padin, S., Plagge, T., Pryke, C., Reichardt, C. L., Rest, A., Ruel, J., Ruhl, J. E., Saliwanchik, B. R., Saro, A., Schaffer, K. K., Shaw, L., Shirokoff, E., Song, J., Spieler, H. G., Stalder, B., Stanford, S. A., Staniszewski, Z., Stark, A. A., Story, K., Stubbs, C. W., Vieira, J. D., Vikhlinin, A., and Zenteno, A. (2011). ApJ, 738:139.

Wojtak, R., Hansen, S., and Hjorth, J. (2011). Nature, 477:567-569.

Wright, E., Bennett, C., Gorski, K., Hinshaw, G., and Smoot, G. (1996). ApJL, 464:L21.

Zeldovich, Y. (1972). MNRAS, 160:1P.

Zhao, H., Peacock, J., and Li, B. (2013). Phys. Rev. D, 88:043013. 